\title{
Communicating to Resolve the "Mommy Wars": Testing Communicated Stereotypes and the Common Ingroup Identity Model with Stay-at-Home and Working Mothers
}

Kelly G. Odenweller

Follow this and additional works at: https://researchrepository.wvu.edu/etd

\section{Recommended Citation}

Odenweller, Kelly G., "Communicating to Resolve the "Mommy Wars": Testing Communicated Stereotypes and the Common Ingroup Identity Model with Stay-at-Home and Working Mothers" (2015). Graduate Theses, Dissertations, and Problem Reports. 6340.

https://researchrepository.wvu.edu/etd/6340

This Dissertation is protected by copyright and/or related rights. It has been brought to you by the The Research Repository @ WVU with permission from the rights-holder(s). You are free to use this Dissertation in any way that is permitted by the copyright and related rights legislation that applies to your use. For other uses you must obtain permission from the rights-holder(s) directly, unless additional rights are indicated by a Creative Commons license in the record and/ or on the work itself. This Dissertation has been accepted for inclusion in WVU Graduate Theses, Dissertations, and Problem Reports collection by an authorized administrator of The Research Repository @ WVU.

For more information, please contact researchrepository@mail.wvu.edu. 
Communicating to Resolve the "Mommy Wars": Testing Communicated Stereotypes and the Common Ingroup Identity Model with Stay-at-Home and Working Mothers

Kelly G. Odenweller

\author{
Dissertation submitted to the \\ Eberly College of Arts and Sciences \\ at West Virginia University \\ in partial fulfillment of the requirements \\ for the degree of
}

Doctor of Philosophy

in

Communication Studies

\author{
Christine E. Rittenour, Ph.D., Chair \\ Megan R. Dillow, Ph.D. \\ Aaron Metzger, Ph.D. \\ Scott A. Myers, Ph.D. \\ Keith Weber, Ph.D.
}

Department of Communication Studies

\author{
Morgantown, West Virginia \\ 2015
}

Keywords: intergroup communication, stay-at-home mother, working mother, social identity, categorization, common ingroup, stereotypes, attitudes

Copyright 2015 Kelly G. Odenweller 


\title{
ABSTRACT \\ Communicating to Resolve the "Mommy Wars": Testing Communicated Stereotypes and the Common Ingroup Identity Model with Stay-at-Home and Working Mothers
}

\author{
Kelly G. Odenweller
}

Toward empirically validating motherhood as an intergroup context and uncovering communicative solutions to resolving the cultural "mommy wars," the goal of this dissertation was to test foundational intergroup communication theories with stay-at-home mothers (SAHMs) and working mothers (WMs) across two independent experiments. In Study One, participant SAHMs and WMs $(N=529)$ reported on their outgroup attitudes, affective responses (i.e., contempt, admiration, envy, and pity), behavioral responses (i.e., active/passive facilitation, active/passive harm), intergroup anxiety, and willingness to communicate after viewing a description of a target SAHM or WM designed in accordance with the most prevalent stereotypes of SAHMs and WMs established by previous research. The results of Study One revealed the powerful — and primarily negative - effects of stereotypes on mothers' cognitive, affective, and behaviors responses to outgroup mothers. In Study Two, participant SAHMs and WMs $(N=154)$ reported on their stereotype reliance, outgroup attitudes, perceptions of outgroup typicality, provision of help, and willingness to communicate after viewing an introduction message from a target SAHM or WM designed in accordance with the four representational mediators in the common ingroup identity model (Gaertner, Dovidio, Anastasio, Bachman, \& Rust, 1993; Gaertner, Rust, Dovidio, Bachman, \& Anastasio, 1994). Given that CIIM tenets were not supported when applied to this novel experimental design, the results of Study Two demonstrate the complexities of communicating social categorization and present opportunities for future investigations of the CIIM. The discussion highlights the implications and contributions of these findings to intergroup communication theory and scholarship, mothers' interpersonal relationships, and women's group vitality. Recommendations for future investigations of mothers' intergroup conflict within media coverage of the cultural "mommy wars," across interactions in more developed mother-mother relationships, and via mothers' interactions in online channels are also discussed. 


\section{TABLE OF CONTENTS}

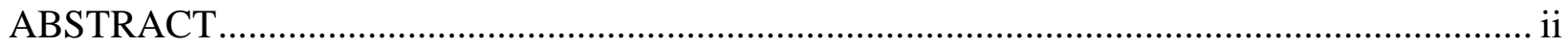

LIST OF TABLES .................................................................................................. vi

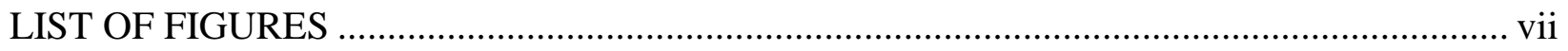

ACKNOWLEDGEMENTS ...................................................................................... vii

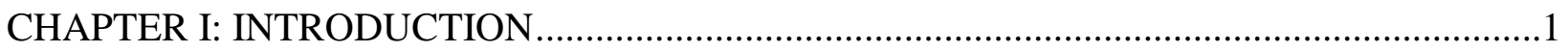

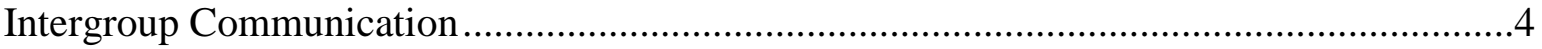

Social Identity and Categorization....................................................................... 10

Social Identity Theory.............................................................................. 10

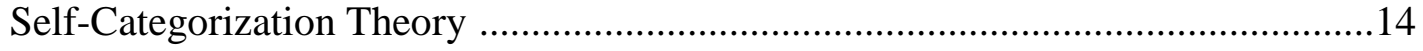

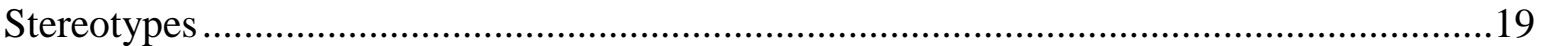

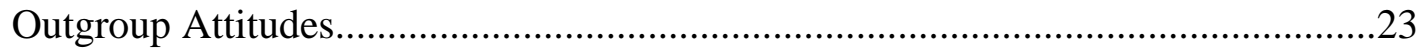

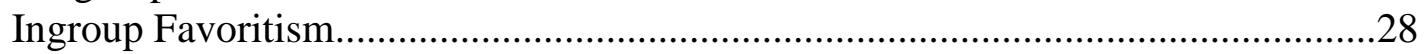

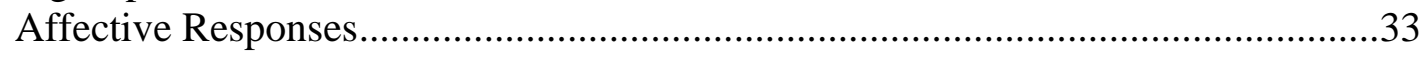

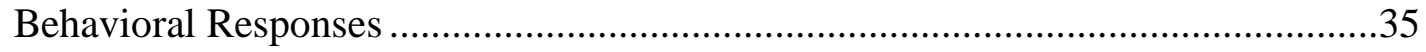

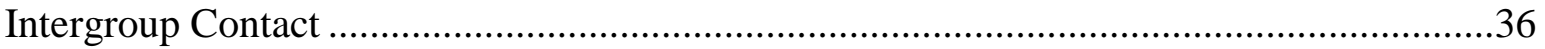

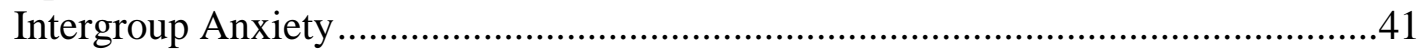

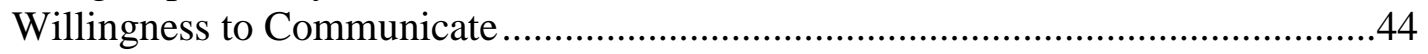

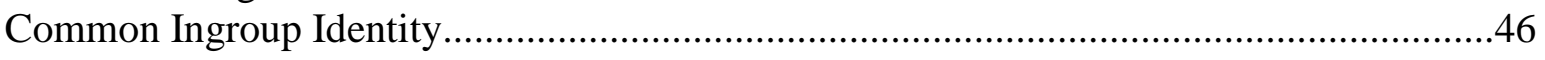

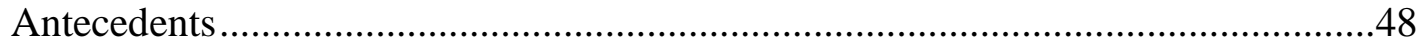

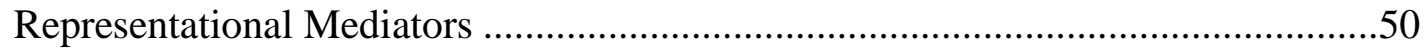

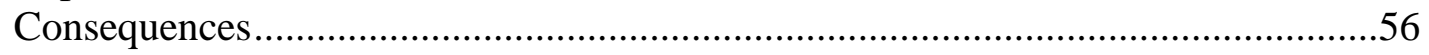

Cognitive Consequences ....................................................................56

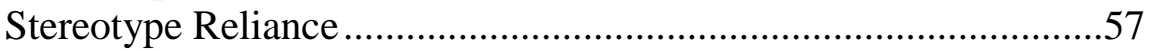

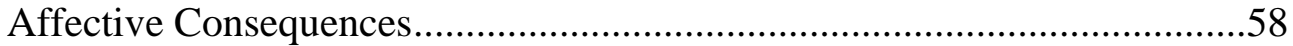

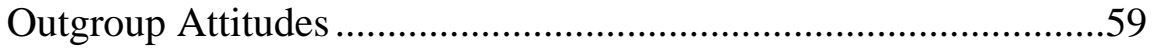

Outgroup Typicality ...........................................................60

Behavioral Consequences ............................................................62

Helping ...................................................................62

Willingness to Communicate ..................................................63

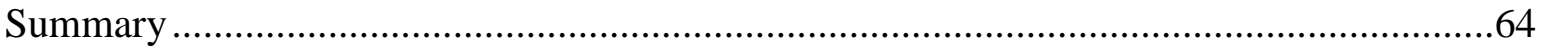

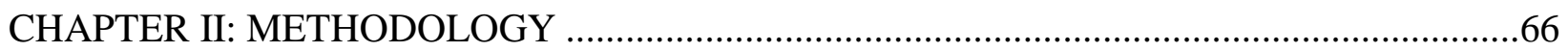

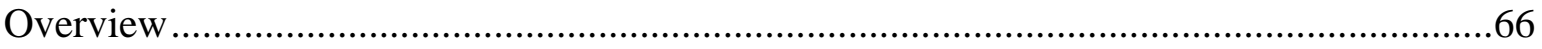

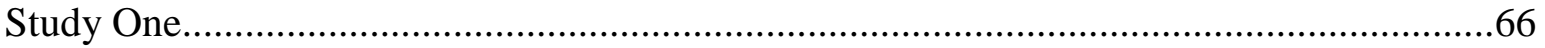

Development of Stereotype Conditions .......................................................66

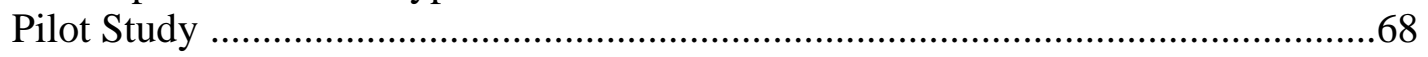

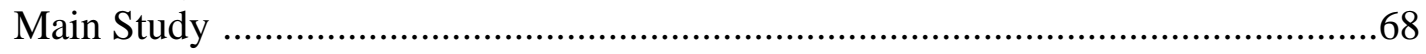

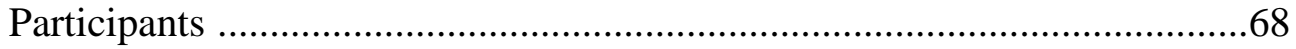

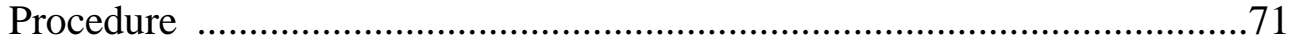

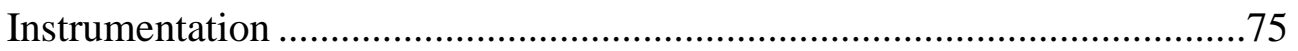




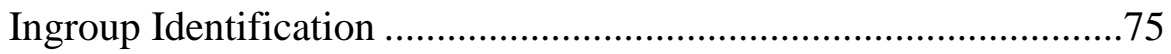

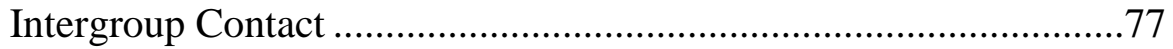

Manipulation Check ....................................................................78

Outgroup Attitudes......................................................................79

Affective Responses....................................................................79

Behavioral Responses ................................................................... 80

Intergroup Anxiety ..................................................................

Willingness to Communicate ............................................................

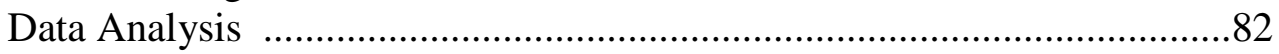

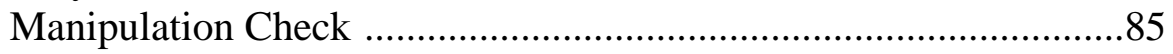

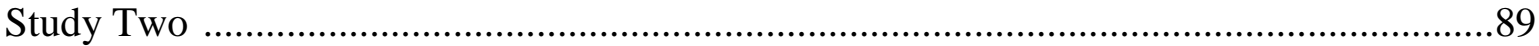

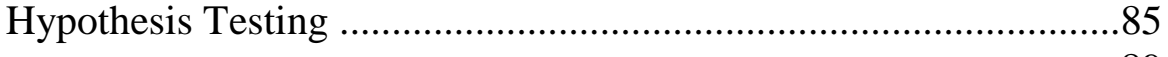

Development of Representational Mediator Conditions .........................................89

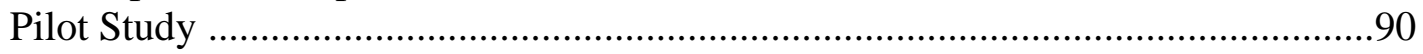

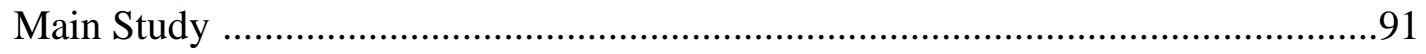

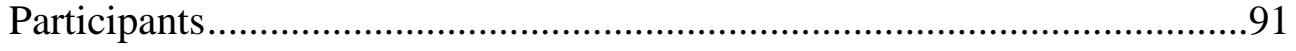

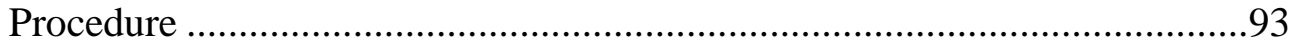

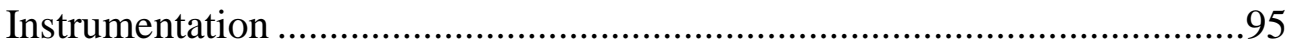

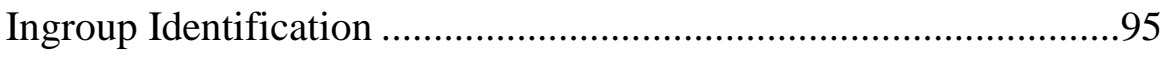

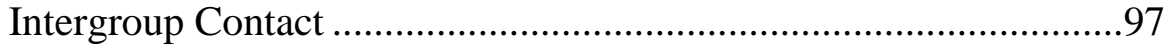

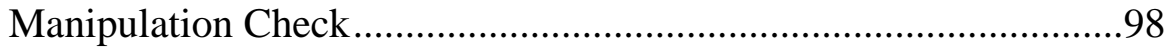

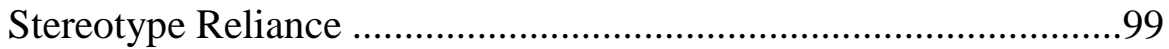

Outgroup Attitudes......................................................................100

Outgroup Typicality ………………………………………........100

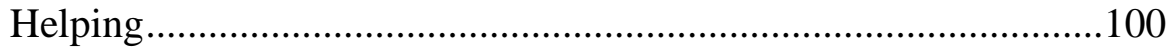

Willingness to Communicate ...........................................................101

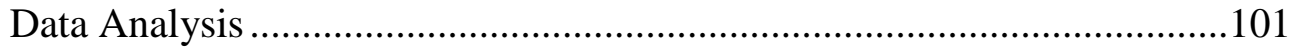

Manipulation Check......................................................................103

Hypothesis Testing ........................................................................103

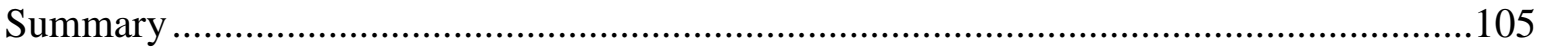

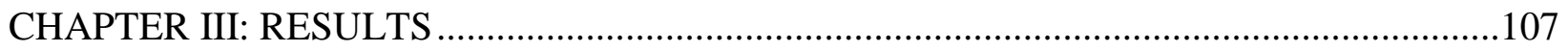

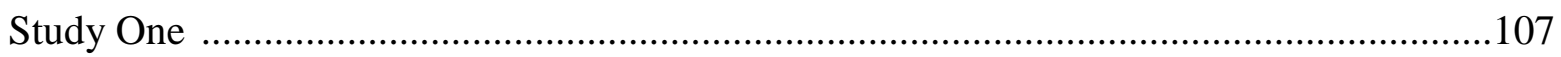

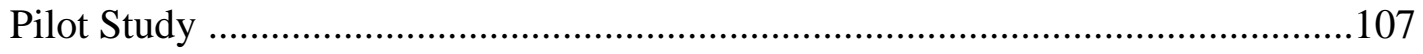

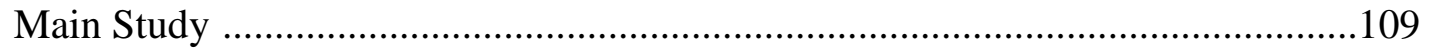

Manipulation Check …………………………….............................109

Hypothesis Testing …………………..................................................109

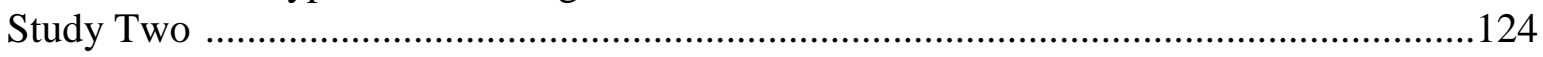

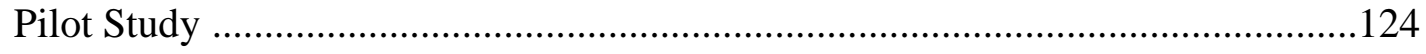

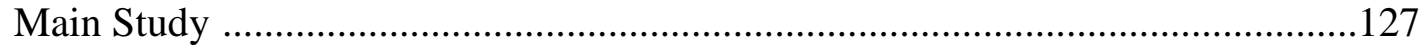

Manipulation Check ………………….................................................127

Hypothesis Testing ………………….....................................................127

Summary .......................................................................................................131

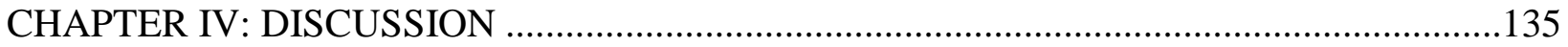

Stereotype Activation..................................................................................................137 
Social Comparison among Stay-at-home and Working Mothers ........................... 139

Hostile Media Effect..................................................................... 143

Group Membership in Initial Intergroup Contact.................................. 145

Mothers' Interpersonal Relationships in Online Contexts.............................. 149

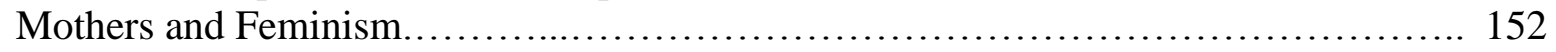

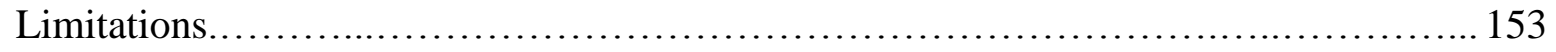

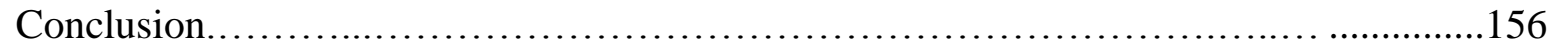

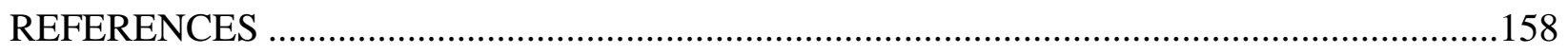

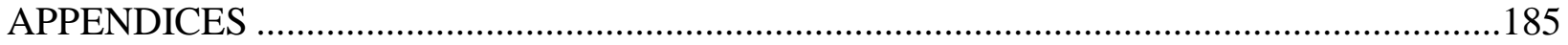

A. Procedures Flow Chart............................................................. 185

B. Study One Stereotype Condition Descriptions................................. 186

C. Study One Pilot Study Questionnaire............................................... 191

D. Online Recruitment Announcement................................................... 193

E. First Response Email to Participant Mothers.................................... 194

F. Second Response Email to Participant Mothers..................................... 195

G. Study One Online Questionnaire................................................... 197

H. Comma Mommas Facebook Group.................................................... 213

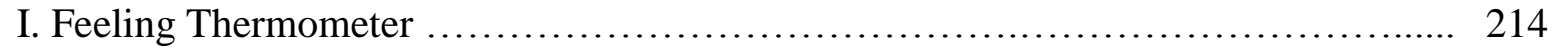

J. Representational Mediator Conditions.............................................. 215

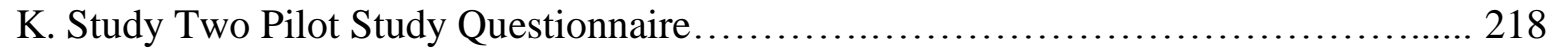

L. Study Two Online Questionnaire.............................................. 220 


\section{LIST OF TABLES}

Table 1. Descriptive and Correlation Statistics for Study One Variables.....................76

Table 2. Descriptive and Correlation Statistics for Study Two Variables....................... 96

Table 3. Frequency Distributions of Forced-Choice Responses in Study One's

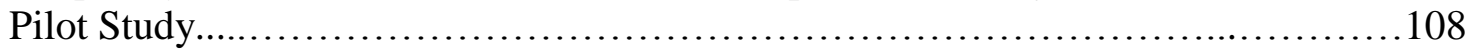

Table 4. Frequency Distributions of Stay-at-home Mother Stereotype Condition Manipulation Checks.................................................... 110

Table 5. Frequency Distributions of Working Mother Stereotype Condition Manipulation Checks...................................................... 111

Table 6. Bonferroni's Adjusted Multiple Pairwise Comparisons for Stay-at-home Mother Stereotype Conditions across Dependent Variables.

Table 7. Bonferroni's Adjusted Multiple Pairwise Comparisons for Working Mothers Stereotype Conditions across Dependent Variables

Table 8. Descriptive Statistics for the Simple Effects Models of Facilitative Behaviors and Intergroup Anxiety across Working Mother Stereotype Conditions for Participant Working Mothers and Stay-at-home Mothers

Table 9. Frequency Distributions of Participant Mothers' Advancement to the Online Interaction.

Table 10. Frequency Distributions of Forced-Choice Responses in Study Two's Pilot Study.

Table 11. Frequency Distributions of Representational Mediator Condition Manipulation Checks.

Table 12. Planned Contrast Results for the Representational Mediator Conditions across Dependent Variables.

Table 13. Descriptive Statistics for Dependent Variables across Representational Mediator Conditions... 


\section{LIST OF FIGURES}

Figure 1. Intergroup-Interpersonal Model (Harwood, Giles, \& Palomares, 2008)............ 7

Figure 2. The Behaviors from Intergroup Affect and Stereotypes (BIAS) Map (Cuddy, Fiske, \& Glick, 2007) ...................................... 22

Figure 3. The Contact Space (Harwood, 2010).................................... 40

Figure 4. The Common Ingroup Identity Model

(Gaertner et al., 1993; Gaertner et al., 1994) 


\section{ACKNOWLEDGEMENTS}

Like all of my accomplishments, the completion (and success) of this dissertation is shared with those who encouraged, advised, and loved me throughout this project. I am incredibly blessed and extremely grateful to have been surrounded by so many amazing people throughout this process.

First and foremost, I must thank my amazing husband, John. I could never have completed this dissertation (and graduate school, in general) if I had not been blessed with YOU as my life partner. You. Are. The. Best. Your unconditional, unwavering, and selfless love and support throughout this journey (which, in total, consumed 5 of our 7 years of marriage and occurred in tandem with the birth of our second child and a million other exciting family milestones!) pulled me through SO many challenging, stressful, and exhausting moments. Thank you for all of the hours (which probably add up to weeks by now) of "single-fathering" you did you so I could bust out some hard-core dissertation work. Thank you for always believing in me, encouraging me, and putting up with me! Thank you for NEVER suggesting I give up and believing all of my hard work would eventually be worth it for me and our family. Thank you for letting me blab on and on about my research and, in the process, allowing me to turn YOU into a "bad-ass feminist bitch," wandering around calling people out on their sexist comments, "mommy wars," and traditional gender values!! You have taught me so much about being a great partner and you have taught our children so much about being a great father. I love you, babe. Now...let's go to Iowa and start a farm!

Next, I must express my deepest appreciation to my children, Leighton and Lochlynn. Thank you for being you. You are both incredibly unique, smart, and kind people who I am just lucky to mother every day. Thank you for being flexible, supportive, and inspiring throughout 
this process. Your hugs, kisses, and down-right hilariousness (Leighton: "Wait. You have been studying to be a doctor this whole time?" Lochlynn: "Happy Birthday, Mommy!" when everyone was congratulating me after my dissertation defense) gave me strength to get through so many difficult times. Thank you for never complaining or making me feel guilty for being a "working mother." Later, when you are chasing your dreams, I hope I am as compassionate and supportive as you have been to me. I hope you look back on this experience our family had and don't remember how busy, stressed, and crazy life was! Instead, I hope your memories are mostly of our family's fun times along the country roads of West Virginia and Pennsylvania; mommy's determination, hard work (which will be more important as you learn more about women's rights and opportunities), and immeasurable love for you; and the wonderful people you started to become here. Next stop? Iowa! (Yes, Leighton. Seriously. Iowa.) Look kids, it's...corn fields!! Iowa, are you sure you're ready for these crazy Odenwellers?!

Next, I must thank my amazing parents and siblings—-both by blood and through marriage - for all of your concern, support, and assistance throughout this project. I truly appreciate all the times you asked me how things were going or called/texted to congratulate me on a minor/major milestone. All of those small gestures meant a lot to me throughout this journey! Special thanks to my mom, Robin, for all your help pre-testing questionnaires, proofreading documents, and recruiting participants...and, of course, all the grocery and kidrelated shopping you did so I could focus on this project. Thank you to my dad, Dick, for all of the times you dropped EVERYTHING to drive down to Morgantown and care for a sick "BoBo" so I could go into the office. I appreciate you both so much!

Next, I must thank the most empathetic, uplifting, and calming person I know, my advisor Dr. Christy Rittenour. I cannot express the magnitude of my appreciation and admiration 
for you. I am so very grateful for your warmth and positivity during this dissertation process. You always knew the best things to say when I was feeling anxious or frustrated (mostly, with IRB...ugh), falling into a writing slump, or needing motivation to push through to the next hurdle. Thank you ALWAYS making time for me - no matter what time of day/night I needed you or what other amazing things you had going on in your life (you know, like working on tenure, raising a family, being a supportive partner, etc. etc.) — and believing in me every step of the way. Thank you for EVERYTHING! Now, I'm off to share all the bad-ass intergroup wisdom you taught me with those cyclones!

Next, I must express my gratitude to my committee members: Drs. Megan Dillow, Aaron Metzger, Scott Myers, and Keith Weber. You have each played a pivotal role in this dissertation (and my graduate education as a whole). Thank you for keeping an open mind about the department's first intergroup dissertation and always challenging me to be the best scholar I could be. I have learned so much from all of you and could not have been surrounded by better mentors! Thank you so much!

Finally, I will end by thanking West Virginia University's Department of Communication Studies as a whole (which includes all of the former and current docs, faculty members, and staff who have left an impact on my life), and its fabulous chair (and my boss over this last year), Dr. Matt Martin. Thank you for giving me the opportunity to complete my degree in this wellrenowned doctorate program. I am elated and humbled to now be included in an impressive line of WVU Communication Studies Ph.D.'s. No matter where my academic career takes me, I will never lose that little piece of me that was once, and always will be, a Mountaineer! 


\section{Chapter I}

\section{Introduction}

Although fathers play a pivotal role in both work and family spheres (e.g., Duckworth \& Buzzanell, 2009; Lamb, 2010), mothers have incited interest among social scientists in a variety of disciplines for decades. Traditionally, mothers have been studied at a micro level in terms of their unique contributions to their families such as through child rearing and socialization, household labor, work/life balance, and influence on child outcomes (see Arendell, 2000; Halpern, 2005; Medved, 2007). Although this body of scholarship is important for understanding mothers', children's, and general family dynamics, opportunities to expand this line of inquiry abound. Thus, this dissertation seeks to examine mothers' communication and wellbeing from a novel intergroup perspective (to be defined in turn) that emphasizes mothers' social identities and membership in different social groups. This unique intergroup view of motherhood draws from theoretical frameworks and empirical research in communication studies, social psychology, and feminist/gender studies. This interdisciplinary approach allows for the study of mothers' interactions in and out of the family (with family and non-family members) as they influence and are influenced by mothers' (gendered) social positions. Motherhood exists as an intergroup context (i.e., between social groups) ripe for empirical investigation for five reasons. First, mothers consider "mother" their most central and satisfying role — over and above those associated with their occupations or romantic partnerships (Graham, Sorell, \& Montgomery, 2004; Rogers \& White, 1998). Second, mothering, defined as socially constructed activities and relational processes that involve caring and nurturing others, is the primary means through which children develop their human identities and understand their societal positions (Glenn, 1994). At the same time, mothering contributes to women's gender 
identity development as mothering predominates views of women (Glenn, 1994; KoropeckyjCox, Romano, \& Moras, 2007). Third, mothers derive much of their self-esteem and mental health (e.g., stress, post-partum depression) from personal, relational partners', and societal evaluations of their maternal role competence (i.e., mothers' ability to fulfill their mothering role by providing child care behaviors that foser child development; Denis, Ponsin, \& Callahan, 2012; Liu, Chen, Yeh, \& Hsieh, 2012; Sun, May 10, 2012; Tarkka, 2003). Fourth, mothers have been situated at the forefront of cultural discourse and empirical scholarship for several decades due to the political and emotional charge associated with mothers' private and public locations (e.g., at home, at work), gender expectations (e.g., traditional/nontraditional, masculine/feminine), and multifaceted roles (e.g., mother, spouse, employee, volunteer, friend; see Arendell, 2000; E. J. Smith, 1981). Fifth, mothers' locations and orientations to mothering and paid employment create divisions and conflicts within the broader social group of mothers (Dillaway \& Pare, 2008). In U.S. popular culture, these have been labeled the "mommy wars" between stay-at-home mothers (SAHMs) and working mothers (WMs; Douglas \& Michaels, February 2000).

Just as the family as a whole represents a powerful intergroup context due to its integration of identity, communication, and socialization (see Soliz \& Rittenour, 2012), the aforementioned phenomena validate the examination of motherhood (one identity within the family) as an intergroup context. Examining motherhood from an intergroup perspective provides an opportunity to explore the influence of the "motherhood" identity on interactions among family members (e.g., partners, children) or individuals outside the family (e.g., coworkers, friends). In turn, a better understanding of links between mothers' identities, communication, and relational quality can be garnered. 
If cultural portrayals of the "war" between SAHMs and WMs (e.g., Douglas \& Michaels, February 2000) are accurate representations of these mothers' relationships, scholarly attention is needed to provide practical solutions for these women in order to preserve their personal and relational wellbeing. Empirical findings derived from foundational intergroup theories such as social identity theory (SIT; Tajfel \& Turner, 1986), self-categorization theory (SCT; J. C. Turner, Hogg, Oakes, Reicher, \& Wetherell, 1987), and the common ingroup identity model (CIIM; Gaertner et al., 1993; Gaertner et al., 1994) suggest ways in which improved communication among these subgroups of mothers (as well as other social groups) can ameliorate antagonistic intergroup dynamics. Thus, this dissertation involves two independent experimental studies to address the (social) psychological and communicative factors surrounding the intergroup dynamics of SAHMs and WMs.

Specifically, the goals of this dissertation are two-fold: to (a) demonstrate the cognitive, emotional, and behavioral consequences to communicated stereotypes of SAHMs and WMs and (b) isolate the effects of social categorization on SAHMs' and WMs' intergroup attitudes and intergroup communication. The following sections discuss the intergroup approach to SAHMs' and WMs' relationships; utilize SIT and SCT to explain the potential influence of social categorization and stereotyping in SAHMs' and WMs' intergroup attitudes, communication, and affective and behavioral responses; and draw upon the CIIM to make predictions about improving attitudes and communication between these subgroups of mothers. Before delving into theoretical underpinnings of this dissertation, a specific definition of intergroup communication is forwarded to frame this dissertation's unique application of identity, social categories, and (interpersonal) communication. 


\section{Intergroup Communication}

Intergroup communication occurs when either person in a social interaction defines

him/herself or the other person in terms of their social group membership (Harwood et al., 2008). Social group membership is rooted in the social categories individuals use to identify ourselves and others. Examples of social categories include age, race, culture, sex/gender, social status, and sexual orientation. These are just a few possible social categories; virtually any social category can be studied as an intergroup context. The only minimal requirement is that the social category include members who are highly invested and derive a shared sense of identity from the group. This emphasis on social categories is what distinguishes intergroup communication from (small) group communication. Instead of viewing communication as an interactional process that occurs between groups (as with group communication), intergroup communication views communication as an interactional process - in any context - that is influenced by (social) group memberships (Harwood et al., 2008).

The groups in which individuals' are highly invested, or ingroups, comprise their social identities. Each individual has multiple social identities that —albeit constantly shifting in salience based on the context (e.g., gender identity is salient in opposite-sex interactions but then overshadowed by the salience of racial identity in interracial interactions) — shape who they are, how they see themselves, and how they view the world around them. The groups with which one maintains identification (i.e., ingroups) are distinct from outgroups, or groups to which individuals to do not perceive themselves to be members. Whereas social identities refer to group categorizations, personal identities are comprised of individuals' idiosyncratic characteristics, unique personality traits, and personal thoughts and feelings that they deem as unique in distinguishing themselves from other individuals (Giles, 2012; Harwood et al., 2008). 
Personal identities are not completely adjacent from social identities as personal identities often include idiosyncratic combinations of social identities and personalized dimensions of social group memberships. In this way, one's idiosyncratic relation to his/her social group and his/her unique combination of social identities is what makes him/her a unique person. Although intergroup scholars have long touted the influence of social identities and personal identities on our communication with others, this dissertation makes an important contribution by exploring SAHMs' and WMs' identities within a specific social context and emphasizing their communicated identities.

From an intergroup perspective, scholars distinguish between intergroup communication and interpersonal communication by highlighting the relevant salience of each in social interactions (Tajfel \& Turner, 1986). As stated previously, when at least one of the interactants thinks of themselves and/or others in terms of social group membership (and, thus, social identities are salient), intergroup communication has occurred. Conversely, interpersonal communication predominates when individuals' focus on their own and the other person's personal identities. Although the specific communication behaviors individuals use in their relationships are certainly important, intergroup scholars take a more macro-level approach to interpersonal communication, considering any message that personalizes or individuates interactants as interpersonal communication. Thus, from an intergroup perspective, the more personalized an interaction becomes, the more interpersonal the interaction becomes. In this sense, intergroup communication and interpersonal communication are negatively correlated—at least in practice as it appears difficult for one message to simultaneously focus on categoryspecific and individualized information (Oakes, Haslam, \& Turner, 1994). However, scholars contend that the intergroup-interpersonal distinction is a matter of emphasis as, at any point of an 
interaction, individuals' social categories or unique qualities can be made more or less salient in the interactants' minds (see Harwood et al., 2008). Given the negative outcomes associated with communicating in terms of group membership (see Allport, 1954; Hecht, 1998b; Oakes, 2008), interpersonal communication is more of an ideal outcome, versus an isolated context, of this approach.

Harwood et al.'s (2008) intergroup-interpersonal model further clarifies the intersections of and distinctions between intergroup and interpersonal communication under this approach. Extending Tajfel and Turner's (1986) conceptualizations drawn along a single continuum, Harwood et al. (2008) placed interpersonal and intergroup communication on two, theoretically distinct continua that allow for both positive and inverse relationships between these communication phenomena. These relationships are represented in Figure 1 by four quadrants and illustrated in the following sections by examples previously offered by intergroup communication experts, editorial examples created specifically for this dissertation, and empirical findings. Expert examples and empirical findings are appropriately cited to differentiate them from editorial examples.

In the first quadrant (lower left), interactions are low on interpersonal and intergroup communication. These interactions are void of all personal characteristics and social categories because the interactants either lack motivation or capability to learn about their interactant partners' individual qualities or group affiliations. Examples of theses interactions include interactions between intoxicated individuals; interactions between people with severe mental disabilities; and interactions between store clerks and customers, during which the customers rush through the sale, talking on their cellphones, and barely acknowledge the clerks. Given that these interactions rarely occur without a severe, mentally-altering substance or disability or, if 


\section{Figure 1.}

Intergroup-Interpersonal Model (Harwood et al., 2008)

Interpersonal Communication

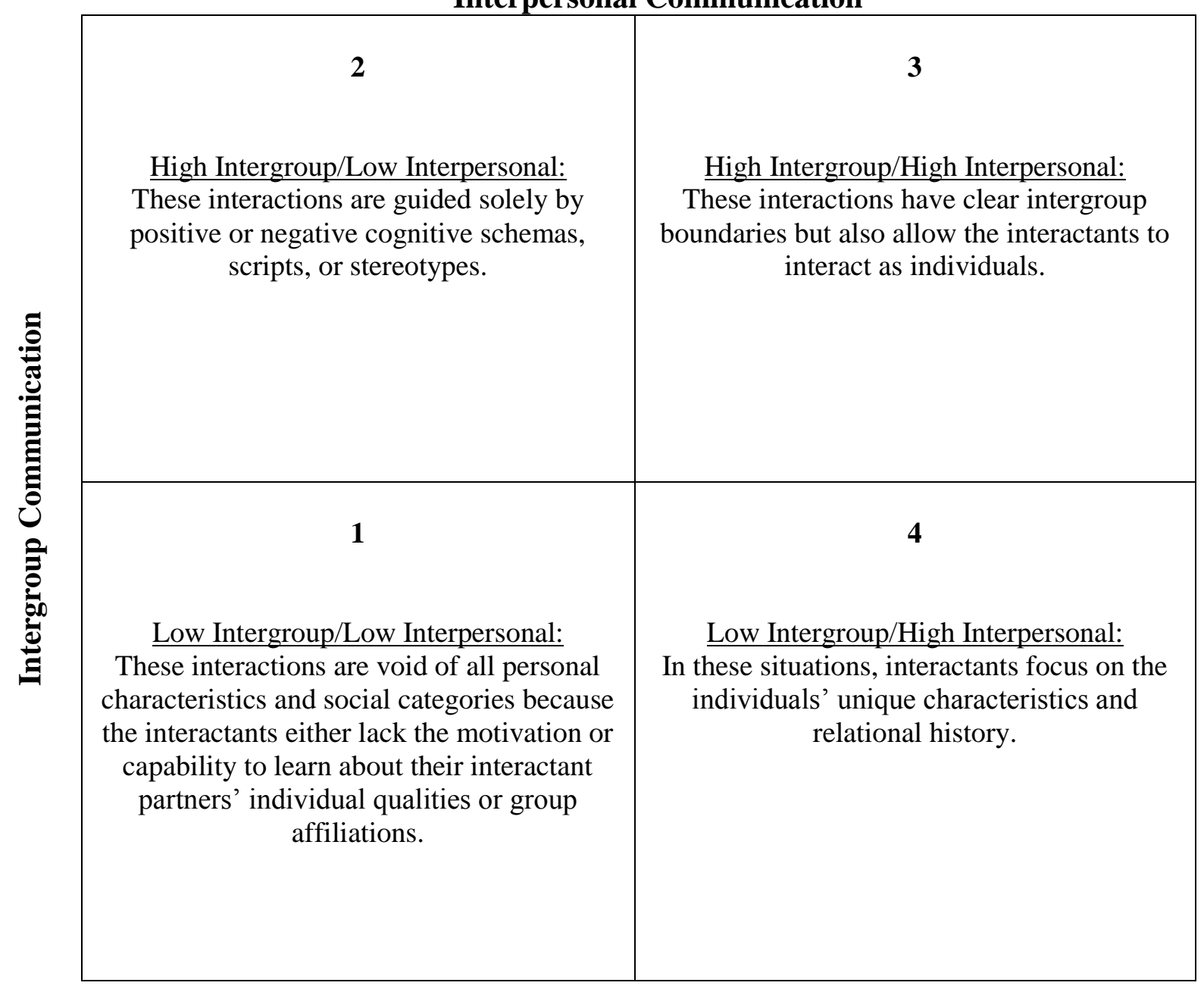


they do occur, they lack substantive messages, these interactions are typically considered unworthy of scholarly inquiry (Harwood et al., 2008).

In the second quadrant (upper left), interactions are low on interpersonal communication but high on intergroup communication. These interactions are guided solely by positive or negative cognitive schemas, scripts, or stereotypes. Examples of these interactions include instructors referring to their Asian students as "math geniuses" because of the cultural stereotype which depicts all Asians as proficient at math (Ruble \& Zhang, 2013); younger adults using slow and loud speech and simple vocabulary when interacting with older adults based on the assumption that all older adults are mentally impaired (Hummert, 1994); and traditional, conservative women confronting feminist women about their presumed abortions and lesbian lifestyle because the media portrays feminists as proponents of legal abortions and gay rights. These examples reflect the general trend of high intergroup/low interpersonal interactions tending to have a negative overarching effect on the quality of the interaction (Harwood et al., 2008).

In the third quadrant (upper right), interactions are high on interpersonal and intergroup communication. These interactions have clear intergroup boundaries (i.e., the groups to which each person belongs are highly salient) but also allow the interactants to communicate as individuals. Examples of these interactions include marital couples arguing about an intimate issue in ways that emphasize gendered stereotypes (Tannen, 1990), children disclosing they are gay to their parents who listens compassionately to their children's feelings but still focus on their children's sexual orientation during the conversation (Harwood et al., 2008), and Catholic colleagues inquisitively asking their Jewish colleagues about their Jewish beliefs and customs in order to better understand their colleagues' religion. Because group memberships are ubiquitous 
and initiated by a variety of prominent or arbitrary social categories (e.g., skin color, family name, favorite sports team), many social interactions involve high intergroup communication as depicted by the second and third quadrants (Harwood et al., 2008). Thus, most intergroup scholarship centers on these areas of the model but, typically they work toward the goal of reaching the fourth quadrant.

In the fourth quadrant (lower right), interactions are high on interpersonal communication but low on intergroup communication. In these situations, interactants focus on both of the individuals' unique characteristics and their relational history. Examples of these interactions include best friends discussing their personal hopes and dreams for the future (Harwood et al., 2008), romantic partners falling in love with unique aspects of one another, and adult siblings fondly recalling memories and feelings from their childhood. Because personal identities influence and are influenced by one's social identities, group memberships are not ignored but, rather, celebrated as part of individuals' uniqueness in these interpersonal interactions. Thus, the aforementioned example with the Catholic and Jewish colleagues could morph into an interpersonal interaction if the interactants chose to decrease focus on their religious identities and place more emphasis on each other's individual characteristics (albeit their individual characteristics are most likely shaped by their religion in some instances but the idea is that these social identities are not influencing the interactants' cognitions or communication behaviors). Although interactions high in group salience (i.e., intergroup interactions) can be positive according to the CIIM (Gaertner et al., 1993; Gaertner et al., 1994) discussed herein, interpersonal interactions are considered ideal as they maximize the personal aspects of the interactants and foster close, intimate, and rewarding relationships among individuals regardless of (or even because of) group membership. Certainly highly interpersonal interactions can also 
be quite negative if interactants accentuate individual characteristics to harm or hurt each other (e.g., Reysen, Lloyd, Katzarska-Miller, Lemker, \& Foss, 2010; Willer \& Soliz, 2010); however, such phenomena are either approached as intragroup phenomena (rankings among individuals within a group) or completely absent in intergroup scholarship. This dissertation draws from foundational and heuristic intergroup theories to explain the conflicts and antisocial communication between SAHMs and WMs.

\section{Social Identity and Categorization}

Intergroup communication cannot be fully defined without articulating SIT (Tajfel \& Turner, 1986) and SCT (J. C. Turner et al., 1987). The major tenets of these theories and their application to this specific context of SAHMs and WMs are discussed in the following sections.

Social identity theory. SIT is one of the most dominant and enduring intergroup theories. In fact, the study of intergroup communication is built upon its principles. Much of intergroup scholarship conducted by post-positivist, interpretive, and rhetorical scholars across academic disciplines centers on SIT's fundamental principles of identity and communication. SIT incorporates various levels of identity and communication to explain the importance of social group categorizations to our sense of self and others, as well as the reasons for intergroup conflict and means of identity restoration.

At the core of SIT is a unique explanation of intergroup conflict. In an effort to combine micro- and macro-level approaches to intergroup conflict (e.g., Allport, 1954; Sherif, 1966) as well as central tenets of intergroup communication discussed previously, SIT claims that intergroup conflict is driven by individuals behaving as members of opposing groups, to which both parties are personally attached, instead of interacting as individual people. Thus, identification, the perception of similarity to and belonging with another individual or group 
(Swann, Gomez, Seyle, Morales, \& Huici, 2009) exists at the crux of SIT’s assumptions, principles, and social change strategies. According to SIT, this group identification motivates individuals to draw ingroup/outgroup comparisons in order to maintain a positive social identity. This claim integrates the three general principles of identity and social comparison from which SIT operates.

The first principle asserts that individuals strive to maintain or enhance perceptions of their personal identities such as self-esteem and self-concepts and social identities such as collective esteem and valued group affiliations. In the absence of intergroup conflict, individuals are able to maintain or enhance positive perceptions of their personal and social identities. In regard to this dissertation's intergroup context, mothering is a salient social identity due to the centrality of this role and feelings of (in)competence and (dis)satisfaction associated with this role (Denis et al., 2012; Graham et al., 2004; Rogers \& White, 1998). Thus, mothers are motivated to preserve favorable perceptions of this identity in order to enhance their self-esteem and mental wellbeing. Odenweller and Rittenour (2014b) provide preliminary support for this notion, demonstrating that SAHMs and WMs strongly identify with their respective ingroups and this ingroup identification is positively related with mothers' group-based esteem.

The second principle posits that every social group and its membership (i.e., ingroup members) are associated with positive or negative connotations. Individuals derive positive or negative evaluations of themselves from the level of their identification with positively- or negatively-perceived ingroups. Positive or negative perceptions of groups are based on groups' level of vitality. Group vitality refers to a group's ability to thrive as a collective group within public (e.g., government, media, institutions) and personal (e.g., marriage, family, social network) spheres of daily living (Harwood, Giles, \& Bourhis, 1994). Group vitality can be 
evidence through groups' size, strength, status, and influence in these particular contexts. As such, group vitality contributes to social hierarchies as groups with stronger vitality are typically awarded more political, economic, and social power (Harwood et al., 1994). Groups with stronger vitality (e.g., younger adults, Caucasians) are also more likely to be accurately represented in the aforementioned contexts, particularly the media and politics (e.g., Abrams, Eveland Jr, \& Giles, 2003; Harwood \& Roy, 1999; Vincze \& Harwood, 2012). Although SIT generally operates from the premise that ingroups are favored over all outgroups (simply because they are not my ingroup), this principle demonstrates how groups' ranking within the social hierarchy influences assessments of groups' favorableness. Positive ingroup evaluations result from perceiving ingroups to be superior or more prestigious than relevant outgroups (i.e., the ingroup compares better), whereas negative ingroup evaluations result from perceiving ingroups to be inferior or less valuable than relevant outgroups (i.e., the outgroup compares better). As illustrated through a traditional intergroup example, poor African Americans may derive positive group distinctiveness by comparing themselves to poor Mexicans and Hispanics-outgroups they consider to be close and, thus, relevant to their social position. Similarly, women have been shown to draw social comparisons with other women because comparing themselves to men, especially in the context of a workplace, evokes feelings of stereotype threat (i.e., the potential to confirm or be reduced to a negative stereotypes associated with one's social group) and role inadequacy (von Hippel, Issa, Ma, \& Stokes, 2011). In the present context, SAHMs and WMs consider their groups socially comparable as both comprise women and mothers and, thus, drawing comparisons with other groups of mothers, instead of with fathers or non-mothers, enables mothers to maintain positive social identities. However, as SAHMs' and WMs' reliance on social comparisons with outgroup mothers increases, their relationships with each other 
become more tense and conflict-ridden.

The third principle claims that, as intergroup conflict (i.e., conflict between different social groups) escalates, individuals' ingroup evaluations and social identities become unsatisfactory, or threatened. Social identity threats occur when individuals perceive themselves as members of devalued or stigmatized social groups (i.e., groups with low vitality; Tajfel \& Turner, 1986). Threatened social identities motivate individuals to employ one of three strategies aimed at restoring positive ingroup distinctiveness. These strategies are social mobility, social creativity, and social competition. Selection of strategy is based on perceptions of alternatives relative to the status quo.

Social mobility refers to disassociation from and/or abandonment of the current ingroup in order to assimilate into a positively-evaluated outgroup and climb the social hierarchy (Tajfel \& Turner, 1986). Individuals can achieve this strategy by gradually making connections and forming relationships with individuals who have higher social status or altering themselves to fit into (or "pass" as) high status, positively-evaluated outgroups (Tajfel \& Turner, 1986). Although self-alterations typically involve changes to physical appearance (e.g., plastic surgery, skin bleaching, new clothing), this strategy can also include linguistic accommodation (e.g., converging to group-specific language) or psychological assimilation (e.g., adopting new attitudes, supporting divergent viewpoints). When individuals perceive constraints to leaving their groups to achieve personal mobility (e.g., access to resources, physical distance, impermeable intergroup boundaries), social creativity is often the next viable alternative (Tajfel \& Turner, 1986).

Social creativity refers to individuals seeking to establish positive distinctiveness for their ingroups by redefining or reframing aspects of their social identities that are subject to intergroup 
comparisons (Tajfel \& Turner, 1986). This strategy need not involve actual changes to social groups' locations or access to resources relative to outgroups (Tajfel \& Turner, 1986). Instead, individuals can achieve this strategy by making ingroups/outgroups comparisons based on new dimensions (e.g., Asian Americans focus on their scholastic aptitude instead of their political disadvantages), altering the values ascribed to group characteristics such that characteristics previously perceived as negative become more positive (e.g., African Americans use the phrase "Black is beautiful" to change negative attitudes about dark skin color), or changing the outgroups such that dominant, high-status outgroups are no longer comparative frames of reference (e.g., lesbians compare themselves to gay men, instead of heterosexual couples, when evaluating their social status; Tajfel \& Turner, 1986). Social creativity is a difficult strategy to employ because negative stereotypes about certain social groups are deeply embedded in our cultural worldviews.

Social competition refers to subordinate group members engaging in direct struggles with dominant outgroups in efforts to achieve equality or gain more recognition for their ingroups (Tajfel \& Turner, 1986). In this sense, power differential is a pre-requisite of social competition as dominant group members rarely consider competition with subordinate groups necessary (Sachdev \& Bourhis, 1985; 1987). Historically, these direct struggles have manifested as civil rights movements, feminist rallies, gay and lesbian activist parades, political lobbying, and language revival movements (Hajek, Abrams, \& Murachver, 2008; Harwood et al., 2008; Sachdev \& Bourhis, 2008). As identity restoration exists outside the scope of this dissertation, these strategies are only included as a backdrop for the broad processes surrounding the specific intergroup context under study.

Self-categorization theory. As an extension of SIT, SCT further explains when and for 
what ends people will categorize themselves and others as individuals versus members of social groups. To this end, SCT is rooted in a process that underlies most intergroup theorizing, including SIT: social categorization. Under SCT, social categorization refers to the process of perceiving and identifying stimuli in the social environment, determining similarities and differences among stimuli, and considering new stimuli in relation to pre-existing cognitive scripts and schemas (Oakes, 2008). As they focused on explaining group identification behavior, Tajfel and Turner (Tajfel, 1978; Tajfel \& Turner, 1986) defined categorization as the cognitive process through which individuals define themselves in relation to others (and their ingroups in relation to outgroups), justify their antisocial behavior, and provide order to social structure and hierarchies.

As SCT was inspired by SIT's primary tenets, SCT's focus on cognitive processes, selfreference, and social contexts can be best understood in relation to SIT. Thus, there are two major comparisons to draw between these two theories. The first distinction between SIT and SCT centers on the role of cognitions in social interactions. Both SIT and SCT place value on cognitive and motivational processes in social interactions but to varying degrees and to achieve different objectives. SCT claims individuals are invariably and simultaneously involved in selfand other- perception and categorization in order to ascertain similarities or differences between themselves and other individuals in their social environments. To this end, SCT accentuates the role cognitions play in establishing social categories and individuals' membership within them. As cognitions about social categories are dynamic and fluid, shifting in the salience from context to context, SCT posits that individuals are motivated to perceive group differences and draw ingroup/outgroup comparisons in nearly every interaction. Thus, under SCT, group membership is primarily a cognitive process. However, the theory's emphasis on fluidity renders 
categorization highly functional for individuals' information processing, social coherence, and uncertainty reduction (Brown \& Capozza, 2000; J. C. Turner et al., 1987)—phenomenon that have powerful communicative and relational consequences, as well. Conversely, SIT emphasizes the functional significance of categorization in terms of distinguishing ingroups from outgroups, providing opportunities for positive group distinctiveness, and cultivating individuals' personal or collective esteem (Tajfel \& Turner, 1986). In this sense, SIT offers a micro-level perspective of cognitive and motivational processes in social interactions. SIT further claims that the goal of intergroup interactions is to minimize (or eliminate) categorization in order to promote personalization and facilitate social change (Tajfel \& Turner, 1986).

The second distinction between SIT and SCT centers on levels of categorization in social interactions. Both SIT and SCT incorporate individualistic and social approaches to group behavior but consider the self and situation to exert different influence on social interactions. According to SCT, the categorization process is specific to the perceiver and context such that the content of individuals' social categories are contingent on their self-perceptions in relation to others (e.g., who I am in relation to who you are?) and the conditions under which meaning is constructed (e.g., where, when, with whom interactions occur; Sachdev \& Bourhis, 2008). At the same time, SCT operates at an abstract, macro-group level by examining the influence of broad, inclusive stereotypes on group distinctions and interactions. Indeed, when social identities are salient during the categorization process, individuals shift from perceiving themselves and others as unique, independent entities (e.g., individuals are proficient at math because they have successfully completed many math courses) to perceiving themselves as members of social groups (e.g., individuals are proficient at math because they are Asian; Harwood et al., 2008). Conversely, SIT does not place as much importance on self-perceptions 
or the social context but, rather, focuses on individuals' evaluations of themselves as group members and their groups' social status (Tajfel \& Turner, 1986). Although distinct, both SIT and SCT add value to intergroup communication scholarship by demonstrating how the cognitive and motivational processes through which we perceive and make judgments based on group memberships influence social interactions.

Communication scholars who apply an intergroup perspective to their research typically study identity and categorization in terms of large, social groups distinguished by arbitrary characteristics such as age, gender, race, and sexual orientation as these are highly salient social identities (e.g., Hummert, Garstka, Shaner, \& Strahm, 1994; Mulac, Giles, Bradac, \& Palomares, 2013; Soliz, Ribarsky, Harrigan, \& Tye-Williams, 2010; Soliz, Thorson, \& Rittenour, 2009). In doing so, these scholars draw more heavily from SIT principles of group identification, group distinctiveness, and social identity threat. With the intent of adding to this literature, this dissertation emphasizes SCT principles, specifically category fluidity, in order to demonstrate the variability in intergroup communication outcomes based on transforming cognitions about outgroup members. To this end, this dissertation is informed by research focused on categorization and subgrouping among females.

When categorizing people based on sex/gender, scholars typically focus on the womenversus-men divide, investigating men and women's perceptions of their gendered social groups in terms of their subgroup structure (e.g., housewives, businesswomen, fathers, career men), stereotype content (e.g., warm, competent), and general favorability (e.g., positive, negative; Vonk \& Olde-Monnikhof, 1998; Wade \& Brewer, 2006). Other scholars, further dividing female social groups, differentiate mothers based on traditional and non-traditional family roles (Brescoll \& Uhlmann, 2005). These trends provide evidence of category fluidity by highlighting 
the shift between large social groups of women/mothers to readily accessible subgroups of these women/mothers. To continue exploring this novel categorization process within a womenversus-women framework, mothers are "further divided" SAHM and WM subgroups to investigate the "war" between these mothers.

The intergroup dynamics among SAHMs and WMs are illuminated by the aforementioned intergroup theories (e.g., SIT, SCT), as well as anecdotal and empirical evidence of SAHMs' and WMs' conflicts. As SIT posits, individuals strive to maintain positive social identities through positive group distinctiveness. Given the constant shift in social identity salience, positive group distinctiveness necessitates subgrouping in certain contexts (Tajfel \& Turner, 1986). For example, when some group members possess qualities that defy the positive image of the group (e.g., poor, lazy, incarcerated), individuals become motivated to divide the superordinate group into smaller subgroups. Subgrouping allows ingroup members to derive a positive identity from their group while avoiding negative perceptions of the broader group. Prominent (subgroup) identities of SAHMs and WMs may be created and/or intensified by women who want to preserve their subgroups' positive social identity without baring the negative aspects of the (broader) social category of mothers. In the media and pop culture texts, this subgrouping manifests as the "mommy wars," a depiction of intense rivalries among mothers based on socially constructed dichotomies of parenting choices such as breast- versus formulafeeding, anti- versus pro-vaccination, and attachment parenting versus the Ferber method (Douglas \& Michaels, February 2000; Zimmerman, Aberle, Krafchick, \& Harvey, 2008). The most prominently depicted rivalry and the focus of this dissertation involves a mothers' role as a paid worker outside the home (i.e., WMs) versus a mothers' role as her children's primary caregiver (Peskowitz, 2005; Steiner, 2007). To empirically validate anecdotal evidence of these 
“mommy wars,” Odenweller and Rittenour (2014, November; 2015, April) recently conducted two investigations of SAHMs and WMs stereotypes. This dissertation extends these studies by utilizing its resulting stereotype profiles to ascertain conditions under which intergroup communication between SAHMs and WMs can foster positive or negative outcomes.

The following sections focus on stereotypes and their influence on social interactions.

First, stereotypes are broadly defined and linked to negative intergroup outcomes. Then, specific SAHMs' and WMs' stereotypes are discussed. Finally, predictions regarding stereotypes' influence on cognitive, affective, and behavioral elements of SAHMs and WMs intergroup interactions are asserted.

\section{Stereotypes}

Although categorization is not inherently negative or the direct result of ignorance (Allport, 1954) and it can be cognitively functional and efficient (Brown \& Capozza, 2000; Oakes, 2008), scholars have come to denounce categorization, labeling it "the root of all evil" (Oakes, 2008, p. 1). When individuals have limited time to process new stimuli from the social environment and lack motivation to critically analyze inconsistencies between new stimuli and pre-existing schemas, negative intergroup outcomes such as stereotypes, attitudes, prejudice, and discrimination result from the "natural" act of categorization (Oakes, 2008; Operario \& Fiske, 2008). The "evil" among groups is often first explored by addressing the stereotypes of specific social categories and their psychological, communicative, and behavioral manifestations.

Stereotypes are generalized and exaggerated "pictures in our heads" associated with social categories that function to simplify cognitive processing of information and enable rationalization of human behavior (Allport, 1954; Lippmann, 1922). Operario and Fiske (2008) argued that stereotypes must be analyzed at multiple levels - cognitive, interpersonal, and 
societal — to truly understand their complexity and universality. Albeit functional to understand the cognitive structures and social context of stereotypes, the content of stereotypes, or "the what, rather than how and why, people think about others" (p. 24), reveals social identities, societal positions, and conflict between social groups (Operario \& Fiske, 2008). Additionally, the interpersonal processes that result when individuals allow stereotypes to influence their interactions can have consequential effects on intergroup relations. Specifically, stereotypes of outgroups reciprocally relate to intergroup communication such that existing stereotypes exert influence on immediate intergroup interactions in terms of attitudes (i.e., positively- or negatively- valenced thoughts and ideas) and prejudices (i.e., inaccurate or unwarranted judgments) about the source of the stereotype (Allport, 1954), new stereotypes develop as a result of intergroup interactions (e.g., Powr, Murphy, \& Coover, 1996), and, together, these stereotypes, attitudes, and prejudices lead to communicative or behavioral manifestations of discrimination.

Discrimination manifests in three ways. First, discrimination manifests as the illusionary correlation effect, a phenomenon that over simplifies the sense-making process about a group and its members by assuming social categories and group behavior are meaningfully—instead of arbitrarily—linked (Oakes, 2008). This effect is more pronounced for negative/extreme behavior and minority groups (Operario \& Fiske, 2008). For example, perceiving HIV/AIDS as a disease to which only gay men are susceptible reflects the illusionary correlation effect because this schema arbitrarily and inaccurately correlates homosexuality with a high occurrence of HIV/AIDS when empirical data reveal different trends for this disease (Prevention, 2011). Judgments and responses based on illusionary correlations are offensive as they reduce individuals to their group memberships and, thus, ignore the differences that make them unique 
individuals. Second, discrimination manifests as ingroup favoritism, or outgroup denigration, whereby social resources are disproportionately allocated in favor of the ingroup and/or denied to the ougroup. These resources may include societal-level privileges such as employment opportunities, access to quality education, and marriage/family rights, as well as relational-level privileges such as listening, social support, and empathy. Third, discrimination manifests as apathy, social aggression and exclusion, and hate speech (Haas, 2012; Harwood \& Joyce, 2012; Willer \& Cupach, 2011). For example, when extremists (e.g., KKK, skinheads, Tea-Party Republicans, Neo-Nazis) use messages that demean, intimidate, or dehumanize their audiences, they deny them basic human rights and limit their access to or silence their voices within social and political arenas. These psychological and communicative consequences to the content of stereotypes incite interest from many intergroup communication scholars (e.g., Hummert et al., 1994; Katz \& Braly, 1933; Ruble \& Zhang, 2013) and inspire the present dissertation.

This dissertation assessed the influence of stereotype content—and associated prevalence and valence — on the cognitive, affective, and behavioral outcomes previously mentioned (e.g., attitudes, prejudice, and discrimination) as they manifest in SAHMs' and WMs' intergroup communication. As such, this dissertation draws upon a specific body of social psychology scholarship that has demonstrated consistent links between cognitive, affective, and behavioral dimensions of intergroup dynamics (e.g., Cuddy et al., 2009; Cuddy, Norton, \& Fiske, 2005; Fiske, Cuddy, \& Glick, 2007; Fiske, Cuddy, Glick, \& Xu, 2002), and has implications for intergroup communication. In one of their recent studies, Cuddy, Fiske, and Glick (2007) developed the Behaviors from Intergroup Affect and Stereotypes (BIAS) Map (see Figure 2) based on an integrative perspective of the mechanisms underlying intergroup dynamics in efforts to explain how individuals' cognitions about a particular group evoke emotional prejudice and 


\section{Figure 2.}

The Behaviors from Intergroup Affect and Stereotypes (BIAS) Map (Cuddy et al., 2007)

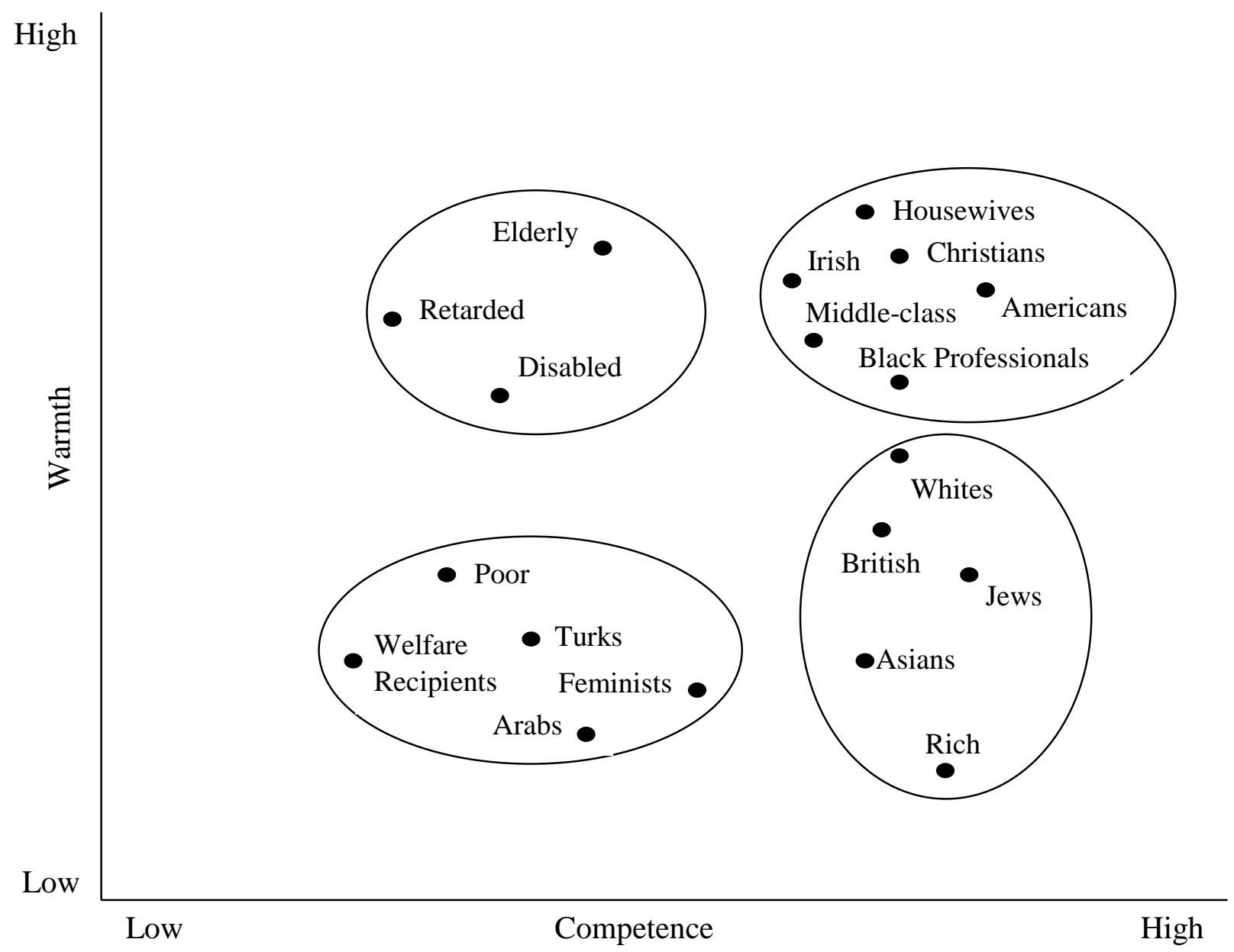

Note: This scatterplot and cluster analysis represents groups' competence and warmth ratings. 
influence behaviors toward the group and its members. The utility of the BIAS Map (Cuddy et al., 2007) rests in its ability to make qualitatively distinct predictions about these four clusters of intergroup behaviors (i.e., active-facilitation, passive-facilitation, active-harm, and passive-harm) based on ambivalent warmth-competent stereotypes (i.e., high warmth predicts active facilitation and low warmth predicts active harm, whereas high competence predicts passive facilitation and low competence predicts passive harm) and ambivalent emotions (i.e., envy elicits passive facilitation and active harm and pity elicits active facilitation and passive harm, whereas admiration elicits active and passive facilitation and contempt elicits active and passive harm). As such, the BIAS map explains how exposure to cultural stereotypes (via the media or learned by family or peer interactions) and their subsequent emotional reactions result in helpful or discriminatory behaviors employed for or against social groups. In this dissertation, SAHMs' and WMs' exposure to cultural stereotypes of these subgroups of mothers will be experimentally manipulated in order to demonstrate the specific effects of stereotype favorability on mothers' responses to outgroup mothers. The components of the BIAS Map that are related to the influence of stereotypes on SAHMs' and WMs' communication are highlighted in the following sections.

Outgroup attitudes. The cognitive component of the BIAS Map involves the link between stereotypes and attitudes. Attitudes represent positively- and negatively- valence thoughts and ideas about a person or group (Eagly \& Chaiken, 1993). Extending general assessments of positive/negative, the BIAS Map provides a powerful assessment of social perception by focusing on universal dimensions of social perception: warmth and competence. All social groups (and their members) can be evaluated based on their friendliness, kindness, and sincerity (i.e., warmth) and social status, competitiveness, and ability to achieve their goals (i.e., 
competence; Fiske et al., 2007; Fiske et al., 2002). The following statements outline the combinations of warmth and competence and the empirical findings of the social groups Americans typically categorize into the listed combinations according to Cuddy et al.'s (2007) work with the BIAS Map. Groups that are viewed as high status and competitive are stereotyped as competent but cold (e.g., Whites, Jews, wealthy people), whereas groups viewed as low status and cooperative are stereotyped as warm but incompetent (e.g., older adults, people with disabilities; Cuddy et al., 2007). Groups that are viewed as low status and competitive are stereotyped as incompetent and cold (e.g., feminists, poor people, Arabs), whereas groups that are viewed as high status and cooperative are stereotyped as competent and warm (e.g., housewives, Black professionals, Christians; Cuddy et al., 2007).

Evidence suggests stereotypes of SAHM and WM mothers exist and elicit powerful attitudes from men and women, parents and non-parents alike — although different trends exist based on the social groups under study. When comparing attitudes toward SAHMs and stay-athome fathers (SAHFs), college students consider these stay-at-home parents to be similar in communal stereotypes (e.g., sensitive, warm, nurturing, dedicated) and parenting effectiveness. However, when comparing attitudes toward WMs and working fathers (WFs), college students consider WMs to be lower in communal stereotypes and parenting effectiveness compared to working fathers (WFs; Bridges, Etaugh, \& Barnes-Farrell, 2002). These trends reveal strict within-gender comparisons based on societal expectations of women's maternal competence. When women embody the prototypical feminine role of the SAHM, they are evaluated as favorably as men in a similar role (i.e., SAHFs) as this role—regardless of gender-begets perceptions of communality and parenting effectiveness. However, when women embody a nontraditional role (i.e., the WM), they are evaluated unfavorably compared to men because men 
are not held to the same femininity or parenting standards at which these women are falling short. Further, male and female adults express considerably more negative affect for WMs and SAHFs compared to SAHMs and WFs (Brescoll \& Uhlmann, 2005). These findings suggest a universal and prescriptive gender role ideology that positions women as the primary caregivers and men as the primary breadwinners and any deviation from this ideology—although SAHFs are considered competent parents (Bridges et al., 2002)—elicits negative societal attitudes. When subgroups of mothers are compared, SAHMs are still evaluated as better care providers, with this trend being more pronounced when young-adult women hold negative attitudes about maternal employment (Shpancer, Melick, Sayre, \& Spivey, 2006).

As previously mentioned, Odenweller and Rittenour (2014, November; 2015, April) conducted two empirical investigations of the content, prevalence, and valence of SAHMs' and WMs' stereotypes as perceived by mothers and non-parents. In the first study, beyond revealing SAHMs' and WMs' identity salience and esteem (mentioned previously), Odenweller and Rittenour (2014, November) uncovered evidence of intergroup conflict between these subgroups of mothers. These findings render SAHMs and WMs a valid social context in which to examine the psychological communicative consequences to ingroup and outgroup categorization. Also in the first study, Odenweller and Rittenour (2014, November) uncovered the content of SAHM and WM stereotypes (i.e., traits, characteristics, and beliefs that describe SAHMs and WMs). The content of SAHMs' and WMs' stereotypes was revealed via rich qualitative data provided by mothers and non-parents (Odenweller \& Rittenour, 2014, November). Using rigorous content analysis procedures (see Hummert, 1994; Ruble \& Zhang, 2013), the qualitative data were coded and collapsed into 43 stereotypes of SAHMs and WMs (Odenweller \& Rittenour, 2014, November). 
In the second study, Odenweller and Rittenour (2015, April) assessed of the prevalence, or prominence, and valence, or (un)favorableness, of the 43 stereotypes revealed in the first investigation. Stereotypes ranged from highly favorable (e.g., loving; dedicated; caring; happy; balancing work, family, and life; hardworking; involved in children's lives) to highly unfavorable (e.g., lazy, uninvolved in children's lives, selfish, aimless, overbearing, overworked, socially isolated, unknowledgeable). Following principal components analyses, the 43 stereotypes were reduced to the 11 most prevalent stereotype profiles of SAHMs and WMs (Odenweller \& Rittenour, 2015, April). As revealed in this study, the most prominent stereotypes for SAHMs are busy and overworked; executive of the home; lazy, selfish, \& lost; ideal mother; balancing work, family, and life; and non-traditional and the most prominent stereotypes for WMs are overextended with no free time, hardworking and determined, flexible and family-oriented, super mom, and traditional. Because stereotypes—in and of themselvesare not inherently negative, and the positive/negative nature of stereotypes is indicative of broad attitudes of the targets of said stereotypes.

Odenweller and Rittenour (2015, April) also demonstrated mothers' and non-parents' attitudes of SAHMs and WMs based on their perceptions of these mothers' warmth and competence. Specifically, SAHMs are perceived as highly warm and moderately competent, whereas WMs are perceived as moderately warm and highly competent. The inconsistencies with "housewives" (i.e., high warmth/high competence) and "feminists" (i.e., low warmth/low competence) on the BIAS MAP (Cuddy et al., 2007) can be explained by the same within-group comparisons previously discussed as the explanatory mechanism behind Bridges et al.'s (2002) findings. When SAHMs and WMs are compared to each other, SAHMs, who are similar to "housewives" given their role within the domestic domain, are not considered as competent as 
WMs; whereas WMs, who are similar to "feminists" given their nontraditional female role but have the mother identity to elevate their warmth scores and female professional identity elevating their competence scores (Cuddy, Fiske, \& Glick, 2004), are not considered as warm as SAHMs.

As comparisons of SAHMs and WMs reveal more cognitive variability in both mothers and non-parents, the next step is to isolate the effects of favorable and unfavorable stereotypes on SAHMs' and WMs' cognitive, communicative, and behavioral responses to each other. Ruble and Zhang's work (e.g., Ruble \& Zhang, 2012, November; Ruble \& Zhang, 2013) provides a strong foundation for predicting the influence of stereotypes on intergroup outcomes in this specific context. In their first piece, Ruble and Zhang (2013) uncovered the most prominent positive (e.g., smart, friendly, quiet) and negative (e.g., oblivious, annoying, bad at speaking English) stereotype profiles of Chinese international college students held by American college students. In their second piece, Ruble and Zhang (2012, November) demonstrate the negative effects of these stereotype profiles on American students' intercultural sensitivity, perceptions of the target Chinese international student's social attractiveness, level of anxiety about meeting, and willingness to meet that Chinese student. Social attractiveness represents the degree to which a person likes, favorably evaluates, and wishes to establish a friendship with another person. Ruble and Zhang (2012, November) demonstrated that negative stereotype profiles (e.g., oblivious/annoying) decreases a target's social attractiveness; whereas, positive stereotype profiles (e.g., smart/hardworking, shy/quiet) increase a target's social attractiveness. As attitudes are likely subsumed under assessments of social attractiveness, these results provide grounds for asserting the following hypothesis in regard to SAHM and WM stereotype conditions:

H1: Controlling for quantity and quality of previous intergroup contact, stay-at-home 
and working mothers' attitudes toward a target outgroup mother will differ across stereotype conditions.

Exploring the phenomenon of ingroup favoritism is a natural next step upon addressing the links between stereotypes and attitudes. Ingroup favoritism is derived from core SIT principles, highlighting the favorable evaluations and treatment extended to members of the groups with which individuals identify. The decision to include ingroup favoritism in this dissertation stems from the fact that there is a plethora of evidence that suggests SAHMs' and WMs' societal positions influence their biased ingroup attitudes.

Ingroup favoritism. As previously mentioned, the intersection of intergroup stereotypes, attitudes, and discrimination can manifest as ingroup favoritism. Ingroup favoritism refers to the tendency to exaggerate positive attributes of, feel positive affect for, and give preferential treatment to members of one's own social groups (Allport, 1954; Hecht, 1998b). Although there is no empirical evidence documenting specific ways that SAHMs and WMs give preferential treatment to their ingroups, intergroup theorizing and empirical research in other contexts, as well as demographic trends of mothers, imply ingroup favoritism pervades these mothers' intergroup relationships. According to SIT, when individuals perceive themselves as part of (or identify with) a social group, they tend to favor that group over the outgroup(s); thus, exhibiting ingroup favoritism (Tajfel \& Turner, 1986). In fact, both men and women describe and evaluate their social groups more positively than they evaluate the social groups in which they do not belong (Vonk \& Olde-Monnikhof, 1998). Compared to men, women generally display stronger ingroup positivity biases (Rudman \& Goodwin, 2004), implying SAHMs' and WMs' susceptibility to ingroup favoritism. Additionally, given the links between ingroup identification, ingroup favoritism, and intergroup conflict under SIT's major principles, ingroup 
favoritism likely explains SAHMs’ and WMs' conflict (Douglas \& Michaels, February 2000; Odenweller \& Rittenour, 2014b).

SAHMs' and WMs' ingroup favoritism is evidenced through their (sub)groups' vitality. As previously mentioned, group vitality refers to groups' ability to thrive as a collective unit within society and gain/maintain social power (Harwood et al., 1994). Given general SIT principles of identity and social comparison, identification-favoritism links, and evidence of SAHMs' and WMs' ingroup identification (e.g., Crisp, Stone, \& Hall, 2006; Odenweller \& Rittenour, 2014b; Tajfel \& Turner, 1986), SAHMs and WMs likely feel a strong sense of pride and personal investment in their (sub)groups' historical and present-day group vitality. In turn, these mothers likely display biases toward their ingroups.

Demographic factors also contribute to group vitality as the sheer number of group members provides support for the group's collective power (Harwood et al., 1994).

Demographic trends regarding mothers' employment and caregiving lifestyle choices illustrate their groups' vitality and, coupled with their evaluations of their lifestyles, suggest ingroup favoritism. According to the U.S. Census Bureau (Laughlin, 2011), 64\% of all mothers in the United States were employed part- or full-time in 2008 compared to only $39 \%$ in 1980 . This trend demonstrates the substantial increase in WMs over the last several decades, suggesting many women favor this family/career arrangement. WMs also tout the benefits to being career women as if to validate their (and other WMs') family and career decisions. Specifically, compared to SAHMs, WMs reporter higher self-esteem and low depression symptoms (Kim \& Wickrama, 2014) and greater life satisfaction (Berger, 2013). Following three decades with a decline in the number of full-time caregivers, a recent Pew Research report highlighted a substantial increase in stay-at-home motherhood (Cohn, Livingston, \& Wang, 2014), 
demonstrating women's revitalized interest in this lifestyle choice. The vast majority (85\%) of SAHMs state they do not work because they are taking care of their home and family, not because they attend school full-time, cannot find a job, or are disabled (Cohn et al., 2014). These trends suggest SAHMs are satisfied with not working. Qualitative interviews with SAHMs share these women's proclamations that they are largely satisfied with and committed to this family arrangement (Rubin \& Wooten, 2007; Secunda, 1995). Taken together, these trends provide a strong foundation on which to assume SAHMs and WMs favor their ingroups. The predictions regarding SAHMs and WMs biased ingroup attitudes are described in the following hypothesis:

H2: Ingroup favoritism will occur such that participating mothers will report more positive attitudes when the target mother is a member of the participating mothers' ingroup compared to when the target mother is a member of the participating mothers' outgroup.

Given that attitudes toward a group, compared to the content of that group's stereotypes, are more predictive of attitudes toward an individual within that outgroup (Wade \& Brewer, 2006) and societal evaluations of SAHMs are generally more favorable than WMs', SAHMs' ingroup favoritism is likely more pronounced than that of WMs. According to traditionalalbeit pervasive and enduring — perspectives of mothers, SAHMs are considered the "ideal mom." The intensive mothering ideology (i.e., the belief that mothers must be exclusively absorbed in activities that protect, nurture, and socialize children) typically associated with SAHMs (Hays, 1996) is considered the "real, natural, universal" (O'Reilly, 1996, p. 88) and "normative standard" (Arendell, 2000, p. 1195) against which all other mothering arrangements are evaluated. Some scholars attribute economic prosperity to intensive mothering as SAHMs' management of child and family matters facilitate their partners' business success (Crittenden, 
2001). Evidence to support this ideal standard of motherhood are messages from actual mothers including, "good moms stay home with their kids," "[motherhood] is the most important job in the world," and "a mother needs to devote her entire life to her children" (Heisler \& Ellis, 2008, p. 453). These messages complement a Pew Research report that revealed 55\% of SAHMs consider staying home full time the ideal family situation — a percentage that has been increasing since 1997 (Cohn et al., 2014; Taylor, Funk, \& Clark, 2007).

Favorable societal evaluations of SAHMs are contrasted with unfavorable societal evaluations of WMs. With this notion of traditional mothers as ideal mothers, the extreme mothering ideology (i.e., the parent approach that involves constant strategizing to limit time away from children and maximize quality family time through intense and focused interactions) typically associated with WMs is considered a "deviancy discourse" (p. 1195) of motherhood (Arendell, 2000). This deviant mothering ideology has endured centuries of societal scrutiny due to its inclusion of women's nontraditional personal, educational, or career aspirations and references to time away from their children (Dillaway \& Pare, 2008). Indeed, advocates of traditional gender roles and family structures have asserted that the woman, much less the mother, is "not supposed to work outside the home, employed by someone else" (Crawford \& Valsiner, 2002, p. 117). Further, 60\% of Americans think children are "better off" (p. 10) when one parent stays home to focus on the family, compared to only $35 \%$ of Americans who think children are "just as well off" (p. 10) when both parents work (Cohn et al., 2014). This sentiment mirrors findings from a Pew Research report (Taylor et al., 2007) that demonstrated only $21 \%$ of WMs consider working full time the ideal mother/family situation, which is down $32 \%$ from 10 years prior.

Although WMs are not a minority group in terms of group size, when compared to the 
dominant and culturally idealistic script of motherhood (i.e., the SAHM), they experience similar marginalization, enduring negative evaluations and social stigmas because they "choose" fulltime employment over full-time childrearing. Specifically, cultural perception illustrates thatby virtue of their paid employment-WMs place less importance on and devote less attention to parenting, outsource childcare to unqualified strangers, and forgo valuable bonding time with their children in order to fulfill selfish goals (Crittenden, 2001; Dillaway \& Pare, 2008; Feder, March 13, 2006; Gorman \& Fritzsche, 2002; Medved, 2009). These trends prevail despite contradictory evidence demonstrating WMs do not actually spend less time with their children compared to SAHMs (Bianchi \& Robinson, 1997), quality of interactions — and not quantity — is responsible for mothers' positive (or negative) effect on children (Kim \& Wickrama, 2014), and mothers' employment status bears little influence on parenting practices and effectiveness (Chatterji, Markowitz, \& Brooks-Gunn, 2013; Mills \& Stevens, 1985). WMs substantiate these negative portrayals by justifying their decision to work (P. Buzzanell et al., 2005) and reporting negative reactions, judgment, and stigma related to their work/family arrangements from family members, friends, other women (including SAHMs), and society as a whole (Dunn, Rochlen, \& O'Brien, 2013).

Because SIT asserts an association between social groups and valenced connotations, individuals' group membership contributes to their self- and ingroup evaluations based on social comparisons with relevant outgroups (Tajfel \& Turner, 1986). When individuals perceive a positive discrepancy between their ingroup and relevant outgroups (i.e., the ingroup compares better), they feel more secure and confident about their personal and social identities. However, when individuals perceive a negative discrepancy between their ingroup and relevant outgroups (i.e., the outgroup compares better), individuals feel less favorably about their personal and 
social identities. As such, negative discrepancies lead to social identity threats, or threats related to individuals' perceptions of themselves that are derived from their memberships in a devalued or stigmatized social group or category (Tajfel \& Turner, 1986). Evaluative portrayals of SAHMs and WMs in society, mainstream media, and scholarship render working mothers the inferior, subordinate group. This marginalization of WMs likely threatens their social identity. Social identity threat has been associated with weakened positivity bias as marginalized groups who have lower collective self-esteem (i.e., feel less secure in their societal positions) are less likely to tout their group's achievements and instead, as a form of hegemony, favor the majority group (Crocker \& Luhtanen, 1990). Based on intergroup theorizing about social identity threat, the following hypothesis is posited in regard to WMs ingroup favoritism:

H3: The ingroup favoritism effect will be more pronounced for stay-at-home mothers than working mothers such that participating stay-at-home mothers' attitudes for target stay-at-home mothers will be more positive than participating working mothers' attitudes for target working mothers.

Because this dissertation aims to demonstrate a wide range of consequences to stereotypes, exploring the affective and behavioral components of the BIAS Map (see Figure 2; Cuddy et al., 2007) is the next step to achieving this goal. As previously mentioned, the BIAS Map provides an integrative framework for explaining the links between social cognition, emotional prejudice, and behavioral response toward outgroup members. In the following sections, the established links between stereotypes, emotions, and behaviors are discussed and specific predictions about these associations in the context of SAHMs' and WMs' stereotype content are forwarded.

Affective responses. Drawing from the stereotype content model (SCM; Cuddy et al., 
2009) and interpersonal social comparison literature (e.g., R. H. Smith, 2000), the BIAS Map illustrates how high/low assessments of groups' warmth/competence, as well as between-group comparisons, generate four emotional responses: admiration, contempt, envy, and pity. Upward social comparisons with groups that are warm and competent (e.g., groups that are proximate to one's ingroup and are successful and powerful) elicit feelings of admiration and pride.

Downward social contrasts with groups that are cold and incompetent (e.g., groups that have broken away from one's ingroup and occupy a proximate outgroup) elicit feelings of contempt. Upward social contrasts with groups (e.g., groups with more power or privileges) that are competent but cold elicit feelings of envy. Downward social comparisons with groups that are warm but incompetent (e.g., groups that have little power and are harmless and nonthreatening to one's social status) elicit feelings of pity.

Previous research that places subgroups of women — albeit not necessarily all motherswithin the BIAS Map inform this dissertation's predictions regarding SAHMs' and WMs' affective responses. Drawing from a large, U.S. sample of men and women from diverse age groups, ethnicities, socioeconomics, and education levels, Cuddy et al. (2007) uncovered societal views of housewives as high warmth/high competence and feminists as low warmth/low competence. Although not synonymous, housewives and feminists are similar to SAHMs and WMs, respectively, as discussed previously. Given that perceptions of housewives' and feminists' warmth/competence assessments elicit feelings of admiration and contempt, respectively, it is likely that valenced stereotype content about SAHMs and WMs will influence these mothers' emotional responses toward each other. Thus, in accordance with the BIAS Map, it is expected that differently-valenced emotions can be triggered by differently-valenced stereotypes about a specific mom. This prediction is presented in the following hypothesis: 
H4: Controlling for quantity and quality of previous intergroup contact, stay-at-home and working mothers' affective responses toward a target outgroup mother will differ across stereotype conditions.

Behavioral responses. The BIAS Map (Cuddy et al., 2007) incorporates the aforementioned cognitive and affective dimensions from the SCM into a framework of activepassive and facilitative-harmful behaviors. Active behaviors are deliberative and effortful behaviors conducted for or against the target group, whereas passive behaviors are less effortful behaviors conducted with or without the target group but that still have consequences for the group. Facilitation leads to favorable outcomes or gains for the target group, whereas harm leads to negative outcomes or losses for the target group. Thus, active-facilitation involves behaviors that help, assist, or defend others (e.g., charitable giving, antidiscrimination laws). Passivefacilitation involves convenient cooperation with others (e.g., forming win-win work groups with outgroup members). Active-harm involves behaviors that explicitly hurt a group or its interests (e.g., employment discrimination, genocide). Passive-harm involves subtle discrimination (e.g., excluding, ignoring, or neglecting certain groups).

Previous research regarding the links between stereotypes, emotions, and behaviors provides justification for asserting links between these phenomena for SAHMs and WMs. As discussed previously, Cuddy et al. (2007) demonstrated that feelings of admiration (associated with housewives) and contempt (associated with feminists) elicit ambivalent behavioral responses. Depending on which characteristic is made more salient, different behaviors will be elicited. For example, if feminists' lack of warmth is emphasized, this group will elicit active harm from the outgroup; whereas if feminists' incompetence is elicited, this group will elicit passive harm from the outgroup (Becker \& Asbrock, 2012; Cuddy et al., 2007). Drawing from 
the reasoning provided previously for the affective component, valenced stereotype content about SAHMs and WMs should exert varied influence on these mothers' behavioral responses. Thus, the following hypothesis is forwarded.

H5: Controlling for quantity and quality of previous intergroup contact, stay-at-home and working mothers' behavioral responses toward a target outgroup mother will differ across stereotype conditions.

Stereotypes incite interest from communication scholars' because stereotypes influence and are influenced by communication. Put differently, stereotypes about groups and its members are constructed within interactions and, then, change group members' cognitive and communicative approach to future interactions. Given communication's powerful role in intergroup contact experiences (i.e., contact is communication), I will now explore the ways that established stereotypes about SAHMs and WMs effect how these mothers approach (or avoid) contact with outgroup mothers. As intergroup contact scholarship inspires this investigation of SAHMs' and WMs' communication, the next section focuses on seminal and more recent research on prejudice and contact before specific hypotheses that link stereotypes of SAHMs and WMs to intergroup contact variables are asserted.

\section{Intergroup Contact}

Allport's (1954) seminal work on prejudice laid the foundation for future intergroup contact scholarship. Prejudice, as mentioned previously, refers to inaccurate or unwarranted judgments about racial groups that influence intergroup interactions (Allport, 1954). These judgments are typically affective and evaluative in nature such that they reflect emotionallyladen beliefs regarding the superiority of one group over another (Hecht, 1998b). Prejudice is inaccurate and unwarranted because it lacks basis in sufficient facts; is derived from 
misconceptions, hearsay, exaggerated stereotypes, or limited personal experience; overlooks individual characteristics of groups; and is resistant to new, conflicting evidence (Allport, 1954). Although not all prejudices are negative (e.g., older adults deserve special accommodations), prejudice is considered unfavorable because it typically consists of adverse or hostile attitudes that have some "functional significance" (p. 12) for the person who holds them (Allport, 1954). Indeed, it may be functional for dominant group members to harbor prejudices in order to vent frustrations about outgroups, uphold prevailing societal norms and hierarchies, and maintain positive group distinctiveness (Aronson, 1992; Hecht, 1998b; Tajfel \& Turner, 1986). Regardless of individuals' motives, however, prejudice leads to aforementioned deleterious outcomes for minority groups such as hate speech, exclusion, discrimination, physical violence, and extermination (Allport, 1954). Thus, Allport's work (and that of intergroup scholars who follow him) has focused on interventions for this pervasive and deep-rooted societal problem.

As one solution to prejudice, Allport (1954) posited the contact hypothesis. The major premise of the contact hypothesis is that positive contact between individuals of different social groups can reduce prejudice. Allport asserted four conditions essential to positive intergroup contact: (a) individuals in the interaction must be of equal status (e.g., peers, coworkers), (b) individuals must be engaged in cooperative (versus competitive) communication (e.g., turntaking, expressing appreciation, recognizing contributions), (c) individuals must be working toward a common goal, and (d) individuals' efforts must be aligned with institutional support (e.g., laws, rules, social norms) outside of the interaction. Since Allport's (1954) conception of the contact hypothesis, many scholars have demonstrated that intergroup contact operating under these four conditions has strong effects on prejudice/conflict reduction (Binder et al., 2009; Hewstone, 2003; Pettigrew, 1997; Schiappa, Gregg, \& Hewes, 2005). 
Criticism of Allport's (1954) contact hypothesis has informed subsequent intergroup contact scholarship. Critics have argued that positive outcomes of contact are simply due to selection bias such that only individuals with low levels of prejudice seek out intergroup contact and their post-contact levels—regardless if they stay consistent or fluctuate-will be low (see Pettigrew, 1997; 1998). Discrediting this skepticism, Pettigrew and Tropp (2006) found that people with little or no choice about their contact with outgroups (e.g., outgroup members forced to live in the same neighborhood, work together at the same company, or interact in a classroom) experience stronger effects in terms of their prejudice reduction compared to people who enter intergroup interactions with complete volition.

There are two possible explanations for the trends related to prejudice reduction and choice. First, pre-existing positive attitudes about outgroup members precede individuals' choice to engage in intergroup interactions and, thus, the positive change between their pre- and post- interaction attitudes is smaller than that of individuals who enter involuntary intergroup interactions with negative attitudes about outgroup members. Second, individuals who choose to interact with outgroup members have high, and perhaps unrealistic, expectations that real-life interactions fall short of meeting and, instead of leaving interactions with fewer prejudices, their negative stereotypes are actually reinforced following unsatisfying contact. Conversely, individuals who are thrust into interactions with outgroup members may be more likely to leave interactions with less negative attitudes if the four conditions (i.e., equal status, cooperative communication, common goals, and authority support) are met during the interactions. These trends reveal the ecological validity of Allport's (1954) claims because they demonstrate that prejudice reduction is not reserved for a few tolerant people who volunteer to help or work with outgroup members but can actually have stronger effects for individuals who wish to avoid 
communication with outgroup members.

Adjacent to support for the ecological validity of the contact hypothesis, Harwood (2010) encouraged scholars to examine contact conditions' practicality and spontaneity in real-life interactions. Harwood argued that, because scholars typically elicit the conditions as a package in forced, laboratory settings, they prohibit their ability to ascertain each condition's individual effect on bias reduction. As a response to this shortcoming, Harwood developed the contact space (see Figure 3). Although testing all that is eloquently prescribed in the contact space is outside the scope of this dissertation, a full overview of this framework will be provided, followed by a description of a variable expected to be important in this dissertation due to its prominent role in the contact space.

The contact space is a parsimonious two-dimensional framework that organizes the conceptual and operational variations - as well as the similarities that have emerged across disciplines-in previous intergroup contact literature (Harwood, 2010). Researchers can use this framework to explain and make predictions about mediators and moderators of intergroup contact (e.g., anxiety, empathy, accommodation, knowledge), position language and communication (i.e., the actual messages exchanged) prominently within the intergroup contact experience, and outline practical ways to reduce prejudices when studying intergroup contact in a variety of communication contexts (e.g., face-to-face, online, media, intrapersonal). The two dimensions of the contact space are the (a) involvement of self and (b) richness of self-outgroup experience. The "involvement of self" dimension refers to how personally involved one is in an intergroup interaction. For example, individuals might directly involve themselves in a face-toface interaction with an outgroup member, observe an interaction between two outgroup members in the media, or imagine themselves in an interaction with an outgroup member-all of 
Figure 3.

The Contact Space (Harwood, 2010)

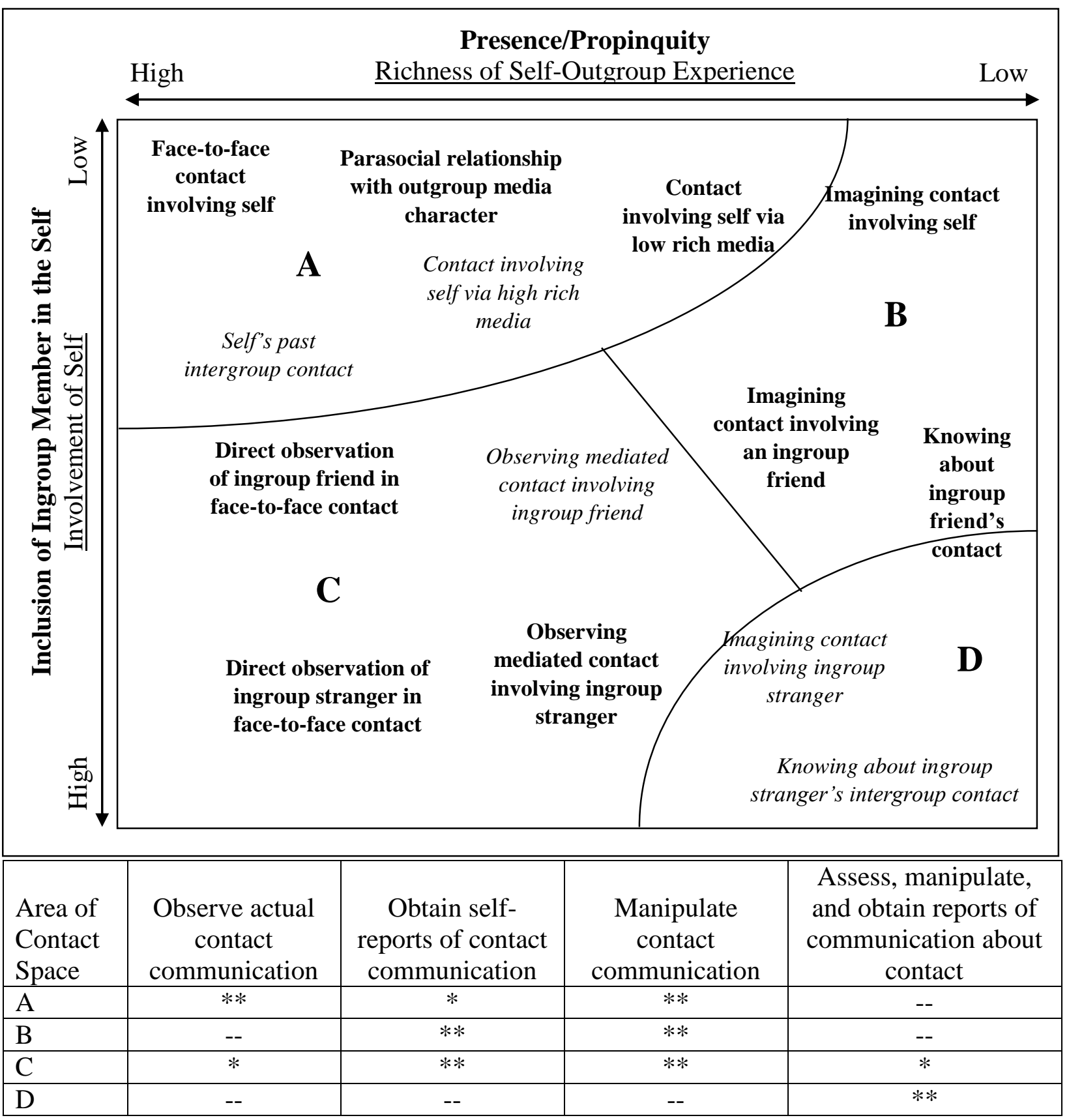

Note. The contact space is a two-dimensional model that accounts for variation in studies of intergroup contact. "Contact" always refers to some type of interaction between an ingroup member (i.e., self, friend, or stranger) and an outgroup member. The areas are labeled with both objective labels (i.e., involvement of self and richness of self in outgroup experiences) and subjective labels (i.e., inclusion of ingroup membership in self and presence/ propinquity). In the body of the contact space, items in bold are areas already studied in the intergroup contact literature, whereas items in italics are contexts of contact that have not previously been examined. In the bottom table, “--" indicates an approach to communication that is unlikely to be possible in the designated area, "*" indicates an approach that is possible in the designated area, and "**" indicates an approach is highly likely to be productive in the designated area. 
which vary in degree of self-involvement. The "richness of self-outgroup experience" dimension refers to the availability of nonverbal cues and temporality of feedback in an intergroup interaction. For example, individuals interacting face-to-face will have access to more nonverbal cues and receive more immediate feedback from their partners compared to individuals interacting via video conferencing, telephone, or text messages. These two dimensions vary as a function of psychological and communicative variables previously established as mediators of intergroup contact. Although many mediators of intergroup contact exist (e.g., empathy, knowledge, accommodation, group norms), the variable shown to be most prominent within the contact space based on its strong influence on intergroup attitudes and stereotype use is intergroup anxiety.

Intergroup anxiety. Intergroup anxiety refers to feelings of fear and apprehension due to the anticipation of negative evaluations and psychological or behavioral consequences when interacting with outgroup members (Stephan \& Stephan, 1985). For example, people fear that outgroup members will perceive them as incompetent, unintelligent, or powerless; outgroup members will take advantage of, manipulate, exploit, or reject them; or their fellow ingroup members will disapprove of their interactions with outgroup members and punish or reject them for associating with the outgroup (Stephan \& Stephan, 1985). As anticipation of these consequences threatens individuals' self-esteem and social identities, individuals report feeling uncomfortable, awkward, frustrated, or irritated in (or even when thinking about) intergroup interactions (Stephan \& Stephan, 1985).

Intergroup anxiety is influenced by a variety of contact, cognitive, and situational factors. First, the amount and quality of, as well as the conditions under which, prior contact with outgroup members has occurred can influence anxiety levels in current and future interactions 
(Stephan \& Stephan, 1985). Interactions between groups with little or no previous contact produce the highest degree of anxiety (Islam \& Hewstone, 1993; Stephan \& Stephan, 1985). Second, cognitions about outgroup members such as pre-existing schemas, lack of knowledge, ethnocentrism, expectations, and uncertainty can influence individuals' feelings about current and future intergroup contact (Stephan \& Stephan, 1985; Stephan, Stephan, \& Gudykunst, 1999). Interactions between groups who are perceived to possess polarizing characteristics (i.e., extreme between-group differences) produce the highest degree of anxiety (Islam \& Hewstone, 1993; Stephan \& Stephan, 1985). Third, many situational factors such as the formality of the interaction, groups' majority/minority statuses, groups' history, or institutional support can affect individuals' fear and apprehension about intergroup contact (Stephan \& Stephan, 1985). Interactions between groups who have a history of conflict produce the highest degree of anxiety (Islam \& Hewstone, 1993; Stephan \& Stephan, 1985). Additionally, negative previous contact increasing anxiety about future contact (Stephan, 2014).

Scholars explore intergroup anxiety more frequently than any other contact variable because it is consistently associated with negative intergroup outcomes (Harwood \& Joyce, 2012). At high levels of intergroup anxiety, individuals tend to exaggerate differences between in- and outgroup members, perceive outgroups as homogenous groups, hold negative outgroup attitudes, and experience negative emotions such as fear and anger (Islam \& Hewstone, 1993; Soliz et al., 2010; Stephan \& Stephan, 1985). Individuals with high intergroup anxiety tend to submit to or reject outgroup members (depending on group status) or avoid outgroup contact altogether (Barlow, Louis, \& Hewstone, 2009; Stephan \& Stephan, 1985).

This dissertation focuses specifically on the link between intergroup anxiety and stereotypes. In addition to the aforementioned consequences, people fear that outgroup members 
will perceive them as prototypes, the average or most typical example (Operario \& Fiske, 2008), of their group's negative stereotypes (Stephan \& Stephan, 1985); thus, reinforcing negative evaluations of their group and threatening their social identity. Unlike fears about strangers and different cultures that are not based in realistic experiences (i.e., xenophobia), intergroup anxiety is highly rational as high levels of anxiety actually do increase individuals' reliance on preexisting schemas and simplified information processing (i.e., categorization) causing them to draw upon negative stereotypes in intergroup contact experiences (Islam \& Hewstone, 1993; Stephan et al., 1999) and reject information that counters existing stereotypes despite groups behaving in non-stereotypic ways (Wilder, 1993). As further evidence of the link between negative stereotypes and intergroup anxiety, Ruble and Zhang (2012, November; 2013) discovered that, not only do American college students hold positive and negative stereotypes of Chinese international college students, American students primed with negative stereotypes about Chinese international students reported the highest degree of anxiety when anticipating contact with a student in the outgroup.

According to Harwood's (2010) position of intergroup anxiety within the contact space, anxiety functions as a strong mediator in face-to-face interactions because physical proximity and expectations for immediate feedback make partners more vulnerable to negative emotions or uncertainty. Anxiety tends to be minimized in computer-mediated interactions because online channels increase physical distance between intergroup partners, give partners greater control over their responsiveness and use of visual cues, and equalize power distribution (AmichaiHamburger, 2012; Amichai-Hamburger \& McKenna, 2006). Despite this assertion, intergroup anxiety likely plays a role in SAHMs' and WMs' mediated contact because these mothers are portrayed as polarized groups in conflict (Douglas \& Michaels, February 2000; Odenweller \& 
Rittenour, 2014b) — factors that accentuate intergroup anxiety — and anxiety has been documented between ethnic/racial outgroups in mediated contexts (e.g., Harwood \& Vincze, 2012; Shim, Zhang, \& Harwood, 2012). Drawing from Ruble and Zhang's (2012, November) findings, SAHMs' and WMs' intergroup anxiety likely differs depending on the valence of the stereotype evoked. Additionally, because contact experiences influence levels of anxiety (Stephan \& Stephan, 1985), it is important to isolate SAHMs' and WMs' previous intergroup contact from the specific effects under study. Thus, the following hypothesis is forwarded:

H6: Controlling for quantity and quality of previous intergroup contact, stay-at-home and working mothers' anxiety about communicating with a target outgroup mother will differ across stereotype conditions.

Although intergroup anxiety is destructive for myriad reasons, anxiety's link with contact avoidance is potentially its most devastating influence on intergroup dynamics. Because contact is the most critical element of prejudice reduction (see Pettigrew, Tropp, Wagner, \& Christ, 2011), we seek ways to improve individuals intentions for contact. To this end, it is essential to examine the influence of valenced stereotypes content on the willingness to communicate with the stereotyped outgroup. This will allow us to explore a communicative consequence to the categorization process.

Willingness to communicate. If the tendency to view outgroups negatively compared to our respective ingroups exists, as SIT posits, it is reasonable to assume individuals would avoid contact with these negatively-perceived outgroups. Many scholars have offered support for this assumption by examining shifts to communication styles (e.g., elderspeak, patronizing talk) based on the valence of stereotypes evoked in intergroup interactions (e.g., Hummert \& Shaner, 1994; Hummert, Shaner, Garstka, \& Henry, 1998). However, only a few studies have provided 
empirical support for the theoretical link between stereotype valence and desired contact. McCann, Dailey, Giles, and Ota (2005) demonstrated that, when young adults perceive older adults through positive stereotypes such as benevolent (i.e., generous, wise, and kind) and personally vital (e.g., strong, active, healthy, flexible), younger adults are less likely to avoid communication with older adults. Similarly, results of Ruble and Zhang's (2012, November) experiment demonstrated that American college students who viewed negative stereotype conditions (e.g., oblivious/annoying) of a Chinese international students, compared to the American college students who viewed positive stereotype conditions (e.g., shy/quiet, nice/friendly), were significantly less willing to meet a target outgroup student. Drawing from these results, SAHMs' and WMs' willingness to communicate with an outgroup mother should differ based on the valence of stereotype viewed. Additionally, because outgroup exposure, regardless of whether or not Allport's (1954) conditions of contact are met, exerts influence on bias reduction (see Pettigrew et al., 2011), it is important to isolate mothers' contact experiences from the specific effects under study. The following hypothesis reflects this prediction:

H7: Controlling for quantity and quality of previous intergroup contact, stay-at-home and working mothers' willingness to communicate with a target outgroup mother will differ across stereotype conditions.

Upon addressing the powerful influence of SAHMs' and WMs' stereotypes on these mothers' cognitive, affective, and behavioral responses, the focus of this dissertation shifts to the powerful influence of SAHMs' and WMs' contact on similar intergroup communication variables. Given that mere contact with outgroup members reduces intergroup bias (see Pettigrew et al., 2011), it is necessary to examine the effects of SAHMs' and WMs' communication on these mothers' intergroup relationships. This dissertation draws upon a 
foundational intergroup communication framework that has been consistently effective in predicting bias reduction (i.e., the CIIM) in order to examine the effects of variations to the social categorization process within intergroup interactions. The next section discusses the CIIM (Gaertner et al., 1993; Gaertner et al., 1994), previous research inspired by this model, and the utility of testing the model in this dissertation.

\section{Common Ingroup Identity}

The CIIM (Gaertner et al., 1993; Gaertner et al., 1994) extends seminal intergroup contact research that demonstrates links between contact with outgroups and improved intergroup relationships, especially—but not only—when it meets four prerequisite conditions of contact (i.e., equal status, common goal, collaboration, and institutional support; Allport, 1954; Pettigrew, 1998). The CIIM provides a unique, integrated framework that explains how prerequisite conditions of contact achieve desired intergroup outcomes. Unlike aforementioned social identity and categorization frameworks (e.g., SIT, SCT) that address categorization as a powerful explanation of —often negative - intergroup dynamics (e.g., group membership, strength of group identification, intergroup conflict), the CIIM positions social categorization at the crux of intergroup contact. In this regard, the CIIM addresses the potential for variations to the social categorization process to create and, more importantly for this dissertation, ameliorate intergroup bias and conflict during intergroup contact experiences.

Consistent with SIT and SCT (Tajfel \& Turner, 1986; J. C. Turner et al., 1987), the CIIM claims that categorizing group members into distinct social groups (i.e., ingroups versus outgroups) during contact cultivates negative intergroup outcomes (e.g., reliance on stereotypes, unfavorable evaluations, prejudice, conflict). What distinguishes the CIIM from other theories and renders it a powerful framework for understanding improved intergroup contact, is its claim 
that inducing group members to conceive of themselves as part of one common ingroup cultivates positive intergroup outcomes (e.g., less reliance on stereotypes, favorable evaluations, prejudice reduction, amicableness). Thus, manipulating the social categorization process during intergroup contact experiences can influence positive interpersonal relationships among outgroup members and facilitate social change for minority groups.

During intergroup interactions (i.e., interactions during which one or more of the interactants consider themselves members of two distinct social groups), manipulating the social categorization process involves two cognitive processes: decategorization and recategorization (Gaertner et al., 1993; Gaertner et al., 1994). Transforming cognitive representations through decategorization and recategorization alters the structure of intergroup contact and this restructuring is a catalyst for reducing intergroup bias and conflict (Gaertner et al., 1994). Decategorization emphasizes group members' individuation. Through decategorization, group members are cognitively disassociated from their group membership(s) and perceived as separate, unique individuals (Dovidio, Gaertner, Hodson, Houlette, \& Johnson, 2005). In turn, intergroup interactions become more personalized, accentuating unique qualities of interactants instead of reducing them to social categories. Personalized interactions are regarded by interactants as satisfying and rewarding (Harwood et al., 2008) and provide a basis for greater appreciation of difference, which is a common goal of intergroup communication scholars' (e.g., Hecht, 1998a). Recategorization emphasizes group members' identification with a common ingroup. Through recategorization, outgroup members conceive of themselves as members of a larger, inclusive group (Dovidio et al., 2005). This is achieved by emphasizing a collective identity among group members or emphasizing subgroup identities within a collective identity. Through recategorization, outgroup members essentially become ingroup members and, thus, 
receive the same favorable evaluations and treatment as existing ingroup members (Gaertner et al., 1994). In turn, this ingroup favoritism engenders more personalized intergroup interactions between once antagonistic outgroup members (Gaertner et al., 1994). In sum, each of these processes work to transform cognitive representations of group membership such that outgroup members no longer perceive each other as members of different groups, but instead, as unique individuals (decategorization) or members of a superordinate ingroup (recategorization).

Although I will focus predominantly on the de/recategorization process in this dissertation, I describe the entire model (see Figure 4) in order to provide an overview of the contact process it seeks to explain. In particular, the CIIM highlights factors that precede contact (antecedents), cognitive variations to group boundaries during contact (representational mediators), and outcomes that follow contact (consequences; Gaertner et al., 1993).

Antecedents. The four antecedents of the model are similar to the prerequisite conditions for positive intergroup contact (Allport, 1954), describing any characteristic of outgroup members' relational history. These include characteristics such as intergroup interdependence, or the degree to which individuals cooperate or compete with one another; group differentiation, or the degree to which individuals perceive similarities between themselves and outgroup members; environmental factors, such as group norms or institutional support that persuades equal or unfair treatment of outgroup members; and pre-contact experiences, or outgroup members' previous exposure to one another in terms of frequency and quality. These conditions of contact have been linked to positive intergroup relations, but research has revealed that transforming group members' cognitive representations of each other (i.e., decategorization and recategorization), as in this dissertation, provides stronger effects on intergroup outcomes (Gaertner et al., 1994). Thus, these antecedents are not the primary focus of 


\section{Figure 4.}

The Common Ingroup Identity Model (Gaertner et al., 1993; Gaertner et al., 1994)
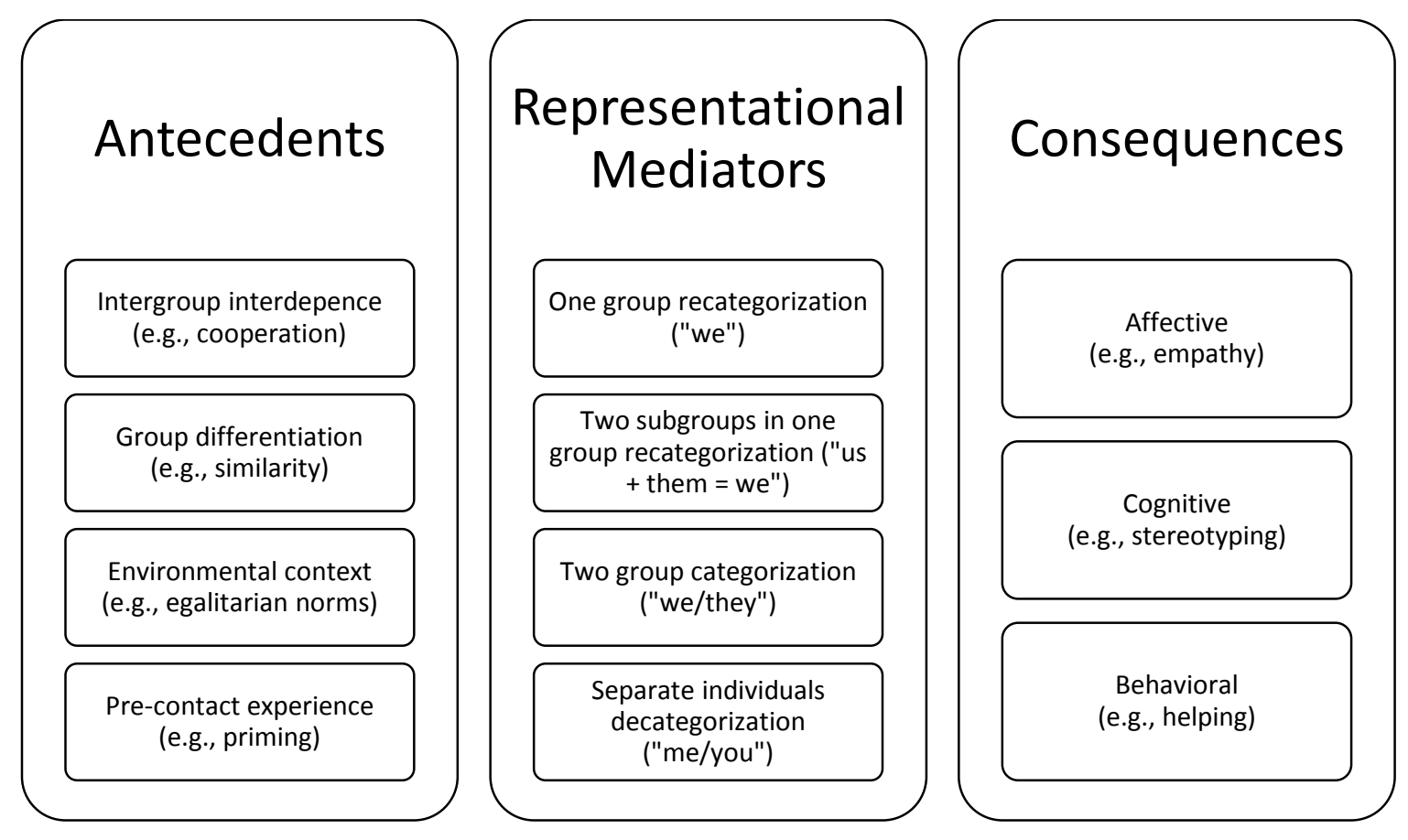
this current proposed investigation of SAHMs' and WMs' stereotypes and contact.

Representational mediators. The social categorization process manifests in the CIIM as four representational mediators: two group categorization, one group recategorization, two subgroups in one group recategorization, and separate individuals decategorization (Gaertner et al., 1994). The two group categorization mediator represents a cognitive process (i.e., social categorization) through which individuals conceive of themselves as members of two (or more) distinct groups. Individuals who cognitively process group membership through this mediator conceive of themselves as ingroups and outgroups, "us" versus "them," or "we" and "they." Examples of this representational mediator from previous studies include college student participants divided into different experimental groups upon arriving at the research lab (e.g., Gaertner et al., 1999; Gaertner, Mann, Murrell, \& Dovidio, 1989); White, Black, Asian, and Hispanic high school students (Gaertner et al., 1994); secular and religious Israeli Jews (Bizman \& Yinon, 2000); United States and Korean citizens (S. Lee, 2005); Asian, Hispanic, and Black Americans (Craig \& Richeson, 2012); Spanish students from two different high schools (Gómez, Dovidio, Huici, Gaertner, \& Cuadrado, 2008); Black patients and non-Black physicians (Penner et al., 2013); White and Black virtual avatars (Vang \& Fox, 2014); and Asian, Hispanic, and Black family members (Soliz et al., 2009).

The one group recategorization mediator represents a cognitive process (i.e., recategorization) through which individuals conceive of themselves as members of one large, inclusive group. Individuals who cognitively process group membership through this mediator conceive of themselves as members of one group (e.g., "us," "we"), minimizing (or even ignoring) group members' unique social identities in order to emphasize a common ingroup. Adding to the examples used above, this representational mediator has appeared in previous 
studies in the following ways. It should be noted that not all researchers induced recategorization but rather assessed its presence. Gaernter and colleagues (e.g., Gaertner et al., 1999; Gaertner et al., 1989) prompted college student participants, previously divided into different experimental groups, to consider themselves members of one group working on the same problem-solving task. Gaertner et al. (1994) investigated high school students with different ethnic backgrounds, assessing the degree to which they think of themselves as students who attend the same northeastern United States high school. Bizman and Yinon (2000) asked religious and secular Israeli Jews to indicate the degree to which they feel as if they are "all in the same boat" (p. 592). Lee (2005) paired U.S. and Korean citizens and assessed their perceptions of belonging to the same negotiation team. Gomez et al. (2008) manipulated perceptions of Spanish students from two different high schools to emphasize a common student identity. Craig and Richeson (2012) induced a "disadvantaged racial minority" identity among Asian, Hispanic, and Black American. Penner et al. (2013) manipulated information Black patients received from their non-Black physicians to enhance perceptions of commonality on the same patient-centered team. Vang and Fox (2014) altered the degree to which virtual avatar teammates competed or cooperated on a problem-solving task. In the family context, Soliz et al. (2009) assessed multiethnic family members' sense of shared family identity (i.e., the degree to which they felt like one united familial ingroup).

The two subgroups in one group recategorization mediator represents a cognitive process (i.e., recategorization) through which individuals conceive of themselves within one large, inclusive group (just as above) and maintain different subgroup identities. Individuals who cognitively process group membership through this mediator conceive of themselves as unique subgroup members working together on the same team (i.e., us + them $=$ we); thus, emphasizing 
(and celebrating) group members' unique social identities within a common ingroup. Adding to the categorization and decategorization examples used previously, this representational mediator has appeared only once in research as college student participants made aware of their membership in different experimental groups while working on the same problem-solving task to earn money (Gaertner et al., 1999).

The separate individuals decategorization mediator represents a cognitive process (i.e., decategorization) through which individuals conceive of themselves as distinct individuals. Individuals who cognitively process group membership through this mediator conceive of themselves as unique people (i.e., you and me), minimizing group members' unique social identities in order to emphasize their unique personal identities. Adding to the categorization and recategorization examples used previously, this representational mediator has seldom appeared in previous research as: college student participants independently working to solve a problem during a research experiment (Gaertner et al., 1999; Gaertner et al., 1989) and separate individuals who do not belong to any particular group at a northeastern United States high school (Gaertner et al., 1994).

Research has provided some insight into the mediators' effects on intergroup bias. Because the two groups categorization mediator acts as a type of "control group," through which the interactants communicate as their usual outgroups, these groups gain no benefits related to ingroup status. In fact, the two groups categorization mediator has been consistently shown as the least successful cognitive process in terms of reducing intergroup bias. For example, participants induced to conceive of themselves as part of two separate experimental groups (i.e., over- and under- estimators on an estimation task), reported less favorable evaluations about, disclosed less intimate information to, and were less willing to help outgroup members when 
compared to participants induced to conceive of themselves as one group (Dovidio et al., 1997). These trends validate the social categorization process as "the root of all evil in intergroup relations" (p. 1) due to its influence on stereotypes, prejudices, and discrimination (Oakes, 2008). Conversely, the strongest effects result from the one group recategorization (i.e., "we"), two subgroups in one group recategorization (i.e., us + them $=w e$ ), and separate individuals decategorization (e.g., "me/you"), when compared to the two groups categorization. For example, participants induced to conceive of themselves as one group or separate individuals while completing a problem-solving task, reported more favorable attitudes toward (former) outgroup members compared to participants induced to conceived of themselves as two separate groups (e.g., Gaertner et al., 1989; Gaertner, Mann, Dovidio, Murrell, \& Pomare, 1990; Gaertner et al., 1994). Although shown to be equally influential on intergroup contact, re- and decategorization (via the one group or two subgroup in one group mediators) reduce bias in distinct ways. When individuals recategorize themselves and outgroup members into one, inclusive ingroup, bias is reduced by increasing the attractiveness of outgroup members (as they are now redefined as members of a superordinate group); whereas, when individuals decategorize themselves and others as unique individuals, bias is reduced by decreasing the attractiveness of ingroup members (Gaertner et al., 1989).

This dissertation's elicitation of the representational mediators is conceptually and operationally distinct from previous investigations of the CIIM. The three distinctions center on: (a) communicated de/recategorization, (b) online context, and (c) all four representational mediators. First, this dissertation is unique because it focused on communicated de/recategorization. The "contact event is a bit of a black box" (Harwood, 2010, p. 165) insofar that scholars know few specifics about the messages exchanged during contact experiences. In 
previous CIIM studies, researchers induced re/decategorization in ways that only tangentially included communication in laboratory settings. These manipulations include proximate/distant seating arrangements, similar/different colored T-shirts, similar/different incentives for completing a task, arbitrary group/personal names (Dovidio, Gaertner, \& Validzic, 1998; Gaertner et al., 1999; Gaertner et al., 1989; Gaertner et al., 1990). Drawing upon Harwood's (2010) "black box" of intergroup contact, when participants in previous studies were encouraged to interact to collaboratively complete a problem-solving activity (and, thus, conceive of themselves as members of a superordinate group), the content of participants' interactions were not examined. In this sense, interaction was one of many elements—but not the focus — of these investigations of intergroup contact (Gaertner et al., 1999). As this has been a consistent trend within intergroup research, scholars have recently advocated for more work that examines actual interactions between outgroups in order to extend our knowledge of intergroup communication (Harwood \& Joyce, 2012). Thus, to provide a communicative application of CIIM, this dissertation emphasized the role of categorization within actual interactions.

Second, the elicitation of re/decategorization in this dissertation occurred within computer-mediated messages between SAHMs and WMs. Harwood's (2010) contact space and scholarly reviews that have merged intergroup and computer-mediated communication frameworks (e.g., Amichai-Hamburger, 2012; Harwood, Hewstone, Amichai-Hamburger, \& Tausch, 2012; Walther, 2009), provided a strong warrant for this design. Harwood (2010) claimed observing actual interaction is best achieved when intergroup contact occurs in face-toface, computer-mediated, or parasocial interactions as these contact experiences allow for high personal involvement, access to nonverbal cues, and opportunities for immediate feedback. Additionally, intergroup scholars (e.g., Harwood et al., 2012) also called for more contact 
research online as online contexts provide unique affordances such as asynchronicity, anonymity, accessibility, and convenience. These affordances facilitate positive intergroup interactions by reducing social distance between diverse populations and providing interactants more control over and time to process their messages (see Amichai-Hamburger, 2012). Because the majority (72.5\%) of mothers' interact in online spaces every day (e.g., Facebook, blogs, online forums; Nielson, 2012), there is a warrant for investigating SAHMs' and WMs' communication in the online contact space. Thus, this dissertation built upon the strong foundation of empirical intergroup knowledge in face-to-face contexts, while capitalizing on the affordances of online interaction, to advance intergroup contact scholarship in online contact spaces and demonstrate practical applications of improved intergroup contact for these subgroups of mothers.

Third, this dissertation extended cross-sectional and experimental research of the CIIM by experimentally testing all four representational mediators within the model. A handful of studies have employed cross-sectional survey designs to investigate the CIIM. In these studies, researchers did not manipulate the representational mediators but instead gathered perceptions of a dual identity or shared family identity to demonstrate links between inclusiveness and improved intergroup outcomes (e.g., outgroup attitudes, self-disclosure, relational satisfaction, constructive conflict management; Bizman \& Yinon, 2000; Gaertner et al., 1994; Soliz et al., 2009). With the exception of Gaertner et al. (1999), experimental tests of the CIIM rarely include all four mediators. Instead, researchers have predominantly examined differences between the one group recategorization and/or separate individuals decategorization compared to the two groups categorization to demonstrate bias reduction following an intergroup contact experience (e.g., Gaertner et al., 1989; Gaertner et al., 1994)—with copious studies relying solely 
on comparisons between two group categorization and one group recategorization (e.g., Dovidio et al., 1998; Gaertner et al., 1989; Gaertner et al., 1990). This dissertation advanced CIIM scholarship by systematically manipulating mothers' social/personal identities based on each mediators' varied depiction of the categorization process. Specifically, dispersed within the computer-mediated message were references to "SAHMs/WMs" for two group categorization, "mothers" for one group recategorization, "SAHMs/WMs" within a larger group of "mothers" for two subgroups in one group recategorization, and "person" for separate individuals decategorization. In implementing this design, this dissertation satisfied researchers' call for experimental tests of the CIIM that demonstrate variability and comparative utility among the four mediators (Gaertner et al., 1994).

Consequences. The CIIM provides a lens through which to examine the effects, or consequences, to transformed cognitive representations. The consequences of the CIIM are organized into three types: cognitive, affective, and behavioral. Within each of the following sections, the research presented demonstrates the links between the two groups categorization mediator and negative intergroup consequences and the one group recategorization, two subgroups in one group recategorization, and separate individuals decategorization mediators and positive intergroup outcomes. These trends provide grounds for forwarding specific hypotheses in terms of SAHMs and WMs intergroup communication.

Cognitive consequences. Cognitive consequences include stereotypes and perceptions of outgroup variability. Following categorization, cognitive consequences for outgroup members include greater reliance on stereotypes (S. Lee, 2005) and perceptions of outgroup homogeneity (i.e., groups members are "all the same"; Gómez et al., 2008). Following re/decategorization, cognitive consequences for former outgroup members and individuals include minimal 
stereotyping (S. Lee, 2005) and perceptions of outgroup heterogeneity (i.e., variability among group members; Gómez et al., 2008). Drawing from cognitive consequences examined by previous CIIM researchers, I plan to explore the effects of each representational mediator on SAHMs' and WMs' stereotype reliance.

Stereotype reliance. As previously mentioned, stereotypes are ambivalent, cognitive belief systems comprised of positive and negative attributes about social groups (Operario \& Fiske, 2008). In two recent studies, Odenweller and Rittenour (2014, November; 2015, April) uncovered 28 stereotypes of SAHMs and 21 stereotypes of WMs, factored into six SAHM stereotype profiles and five WM stereotype profiles discussed previously. In the only study to investigate stereotypes as an outcome of inducing a common ingroup identity, Lee (2005) found that encouraging U.S. and Korean negotiators to conceive of themselves as members of one negotiation team (i.e., common ingroup) led to favorable outgroup attribute judgments (e.g., honest, likeable, intelligent, friendly), whereas high ingroup/outgroup salience (i.e., two group categorization) led to unfavorable outgroup attribute judgments (e.g., greedy, disrespectful, competitive, stubborn).

Another study focused the influence of inducing a common ingroup identity on stereotype threat. According to integrated threat theory (Stephan \& Stephan, 1996), stereotype threat is derived from four components present in intergroup contact experiences: realistic threat (i.e., threats to the ingroup's political, economic, or social power or physical or material wellbeing of ingroup members), symbolic threat (i.e., threats to the ingroup's values, morals, beliefs, and attitudes), intergroup anxiety (i.e., feelings uneasiness, fear, or negativity related to contact with the outgroup), and negative stereotypes (i.e., negative attributes associated with typical ingroup members). All four components are linked to prejudice, with realistic threat 
exerting the strongest effects on prejudice when ingroup identification is high (Bizman \& Yinon, 2001). Although stereotypes and stereotype threat are not synonymous, both phenomena address cognitive attributes of groups and are linked to prejudice (Hecht, 1998b). In their study of stereotype threat and CIIM, Riek and colleagues (Riek, Mania, Gaertner, McDonald, \& Lamoreaux, 2010) demonstrated that inducing a common ingroup identity—either in terms of a shared American identity or high school student identity—among White and Black students reduced stereotype threat and increased positive outgroup attitudes.

Drawing from these findings, SAHMs' and WMs' reliance on stereotypes should vary as a function of the manipulated mediators in the computer-mediated message. As both negatively and positively valenced stereotypes are over-generalized beliefs about social categories that prohibit personalization (i.e., viewing outgroup members are unique individuals) and evoke harmful communication with outgroup members (e.g., harassment, hate speech, exclusion, discrimination; Haas, 2012; Hecht, 1998b; Operario \& Fiske, 2008), mothers' reliance on any stereotypes following de/recategorization will be indicative of intergroup bias. The specific predictions related to stereotype reliance are specified in the following hypothesis:

H8: Following an intergroup contact experience, stay-at-home and working mothers' reliance on stereotypes will differ across representational mediator conditions as put forth in the common in-group identity model such that the conditions that elicit one group recategorization, two subgroup in one group recategorization, and separate individuals decategorization will draw upon fewer stereotypes for the target outgroup mother compared to the condition that elicits two group categorization.

Affective consequences. Affective consequences include attitudes, emotional responses, 
and evaluations of targets. Following categorization, affective consequences for outgroup members include unfavorable attitudes (Gaertner et al., 1994; Gómez et al., 2008; Vang \& Fox, 2014); perceptions of low warmth and competence (Gómez et al., 2008); low empathic concern (Dovidio et al., 1997); low relational satisfaction (Soliz et al., 2009); low trust (Penner et al., 2013); lack of closeness or friendship (Craig \& Richeson, 2012; Gaertner et al., 1989; West, Pearson, Dovidio, Shelton, \& Trail, 2009); and perceptions of interactions as quarrelsome, frustrating, and useless (Gaertner et al., 1989). Following re/decategorization, affective consequences for former outgroup members/individuals include favorable attitudes (Gaertner et al., 1994; Vang \& Fox, 2014); perceptions of high warmth and competence (Gómez et al., 2008); high empathetic concern (Dovidio et al., 1997); perceptions of honesty, cooperativeness, and value (Gaertner et al., 1989; Gaertner et al., 1990); high relational satisfaction (Soliz et al., 2009); high trust (Penner et al., 2013); closeness or friendship (Craig \& Richeson, 2012; Gaertner et al., 1989; West et al., 2009); and perceptions of interactions as cooperative, friendly, pleasant, successful (Gaertner et al., 1989). Drawing from affective consequences examined by previous CIIM researchers, I plan to explore the effects of each representational mediator on SAHMs' and WMs' outgroup attitudes, intergroup anxiety, willingness to communicate, affective responses, and communication satisfaction.

Outgroup attitudes. As previously discussed, attitudes are valenced thoughts about others or social groups (Eagly \& Chaiken, 1993). Attitudes are widely studied across the social sciences, especially by intergroup contact scholars given the links between negative attitudes and stereotypes, anxiety, prejudice, and discrimination (Harwood \& Joyce, 2012; Hecht, 1998b; Pettigrew, 1998). Within CIIM research (e.g., Gaertner et al., 1999; Gaertner et al., 1994), attitudes are influenced by the social categorization process. In particular, perceiving individuals 
as members of two distinct groups (i.e., two groups categorization) is linked to unfavorable outgroup attitudes, whereas perceiving individuals as members of a common ingroup (i.e., one group recategorization and two subgroups in one group recategorization) and unique people (i.e., separate individuals decategorization) is linked to favorable outgroup attitudes. Thus, the following hypothesis predicts differences in SAHMs' and WMs' outgroup attitudes upon viewing computer-mediated messages in which the representational mediators are manipulated:

H9: Following an intergroup contact experience, stay-at-home and working mothers' outgroup attitudes will differ across representational mediator conditions as put forth in the common in-group identity model such that the conditions that elicit one group recategorization, two subgroup in one group recategorization, and separate individuals decategorization will elicit more positive attitudes toward the target outgroup mother compared to the condition that elicits two group categorization.

Outgroup typicality. In order to determine whether outgroup attitudes represent individual- or group-level evaluations and, thus, true bias reduction, it is important to assess outgroup typicality. Outgroup typicality involves both group salience and target's representativeness of the group. In regard to the group salience component, outgroup typicality consists of the degree to which a target's group membership is perceived as salient. Salience refers to the activation or cognitive potency of a social category and/or awareness of the links between social category and target behavior (Oakes et al., 1994; J. C. Turner et al., 1987). Although personalization is highly valued in intergroup interactions because it cultivates an appreciation for diversity that transfers to future contact with the same or new outgroup members (Miller \& Brewer, 1986), group salience is a prerequisite of favorable group evaluations. When 
group salience is low, favorable evaluations likely do not reflect cognitive or affective responses toward a particular group but, rather, reflect idiosyncrasies of the target (e.g., social attractiveness, credibility, liking) or micro-level factors of one's relationship with the target (e.g., relational satisfaction, closeness, trust; Miller \& Brewer, 1986). As a result, intergroup bias toward the group as a whole has not been reduced.

In regard to the representativeness component, outgroup typicality refers to the degree to which the target is perceived as representative of their social group (Hewstone \& Brown, 1986). Stated differently, typicality suggests the target is a "good fit" for the category prototype due to possessing characteristics that are "typical" of that group (Oakes, 2008). Thinking that an intergroup encounter involved a group member who was "an exception to the rule" leaves the general stereotype of the group intact (L. Johnston \& Hewstone, 1992). Changes to attitudes and stereotypes are more likely to occur when individuals perceive group membership as salient and outgroup members as typical group members compared to rare exceptions within their groups (Brown, Vivian, \& Hewstone, 1999). Changing perceptions about the "typical" group member and, thus, disconfirming pre-existing schemas, can foster positive attitudes and dilute stereotypes related to that group (L. Johnston \& Hewstone, 1992; Oakes, 2008; Operario \& Fiske, 2008). When individuals hold positive attitudes about outgroup members and they perceive these outgroup members to be typical representations of their group, positive attitudes about the individuals will transfer to the entire group (Brown et al., 1999; Oakes, 2008; Operario \& Fiske, 2008) improving intergroup relations on a large scale.

Because outgroup typicality exerts powerful influence on attitude and stereotype change, it is essential to gather SAHMs' and WMs' perceptions of a target outgroup mothers' typicality when examining these mothers' categorization and communication. The following hypothesis 
articulates the expected outcomes in terms of outgroup typicality upon manipulating the representational mediations within an online interaction between SAHMs and WMs.

H10: Following an intergroup contact experience, stay-at-home and working mothers' perceptions of outgroup typicality will differ across representational mediator conditions as put forth in the common ingroup identity model such that the conditions that elicit one group recategorization, two subgroup in one group recategorization, and separate individuals decategorization will elicit stronger perceptions of outgroup typicality for the target mother compared to the condition that elicits two group categorization.

Behavioral consequences. Behavioral consequences include communication behaviors and treatment of others. Following categorization, behavioral consequences for outgroup members include contentious conflict management styles (Bizman \& Yinon, 2000), limited selfdisclosure (Dovidio et al., 1997; Soliz et al., 2009), and unwillingness to help (Dovidio et al., 1997). Following re/decategorization, behavioral consequences for former outgroup members/individuals include supportive communication and identity accommodation (Soliz et al., 2009); high quality self-disclosure based on depth and breadth (Dovidio et al., 1997; Soliz et al., 2009); adhering to physicians' medical instructions (Penner et al., 2013); and offering help (Dovidio et al., 1997). Drawing from behavioral consequences examined by previous CIIM researchers, I plan to explore the effects of each representational mediator on SAHMs' and WMs' helping and communication behaviors toward outgroup mothers.

Helping. Prosocial behavior such as helping, charitable donations, altruism, and humanitarianism has been examined across communication and social psychology scholarship (see Penner, Dovidio, Piliavin, \& Schroeder, 2005). A group of intergroup scholars (Dovidio, 
Allen, \& Schroeder, 1990; Dovidio et al., 1997) explored helping behaviors in the context of the CIIM in order to extend self-report measures of CIIM outcomes and bridge the gap between attitudes and behaviors following the induction of a collective group identity within intergroup interactions. In their studies, Dovidio and colleagues (Dovidio et al., 1990; Dovidio et al., 1997) operationalized helping as agreeing hang posters for a student life survey at various locations across campus (i.e., more campus locations signified greater helping). Their results demonstrated that, compared to the two group categorization mediator, the one group decategorization mediator exerted the strongest effect on helping (Dovidio et al., 1990; Dovidio et al., 1997). Based on these findings, it is likely that transforming SAHMs' and WMs' cognitive representations of one another will influence their willingness to help the outgroup mother. Thus, my predictions related to SAHMs' and WMs' helping behavior following the online interaction in which de/recategorization has been elicited are forwarded in the following hypothesis:

H11: Following an intergroup contact experience, stay-at-home and working mothers' helping will differ across representational mediator conditions as put forth in the common in-group identity model such that the conditions that elicit one group recategorization, two subgroup in one group recategorization, and separate individuals decategorization will elicit more helping behaviors toward the target outgroup mother compared to the condition that elicits two group categorization.

Willingness to communicate. Aforementioned theories and research inform assertions regarding SAHMs' and WMs' communication upon transforming these mothers' perceptions of group status. According to SIT, ingroup members are generally deemed more desirable as compared to outgroup members (Tajfel \& Turner, 1986); thus, we would expect ingroup 
members to also be considered desirable interactants. Thus, it stands to reason that altering SAHMs' and WMs' perceptions of ingroup boundaries will influence these mothers' willingness to communicate with one another. This prediction is articulated in the following hypothesis:

H12: Following an intergroup contact experience, stay-at-home and working mothers' willingness to communicate will differ across representational mediator conditions as put forth in the common in-group identity model such that the conditions that elicit one group recategorization, two subgroup in one group recategorization, and separate individuals decategorization will elicit a greater willingness to communicate with the target outgroup mother compared to the condition that elicits two group categorization.

\section{Summary}

The purpose of this dissertation was to demonstrate the cognitive, affective, and behavioral consequences to communicated stereotypes and transformed categorization within intergroup interactions between SAHMs and WMs. Toward achieving this goal, 12 hypotheses derived from central premises of foundational intergroup theories (i.e., SIT, SCT, and CIIM) were forwarded. Specifically, the first through seventh hypotheses (H1-H7) predicted the varied effects of stereotypes on SAHMs' and WMs' outgroup attitudes, affective and behavioral responses, intergroup anxiety, and willingness to communicate. The eighth through twelfth hypotheses (H8-H12) predicted varied effects of manipulating the CIIM's representational mediators on SAHMs' and WMs' reliance on stereotypes, outgroup attitudes, perceptions of outgroup typicality, provision of help, and willingness to communicate. In addition to framing the cultural "mommy wars" in scientific inquiry, this dissertation extends existing research on social identity, category fluidity, stereotypes, and intergroup contact and provides 
communicative solutions for resolving intergroup conflict between SAHMs and WMs. 


\section{Chapter II}

\section{Methodology}

\section{Overview}

This dissertation's methodology centered on ostensive online interactions between SAHMs and WMs who agreed to participate (referred to as "participant mothers" moving forward) and were randomly assigned to one of two independent, quasi-experimental studies, and fictitious SAHMs and WMs (referred to as "target mothers" moving forward), who were either described in accordance with previously established stereotypes profiles of SAHMs and WMs (Odenweller \& Rittenour, 2015, April) or presumed to have sent an introduction message designed in accordance with the common ingroup identity model's (CIIM; Gaertner et al., 1993; Gaertner et al., 1994) representational mediators. The objective of this methodology was to elicit participant mothers' authentic and candid responses about the target mothers in order to demonstrate the cognitive, affective, and behavioral consequences of stereotyping (i.e., H1-H7 in Study One) and transformed group boundaries (i.e., H8-H12 in Study Two). See Appendix A for a step-by-step flow chart of the procedures in this dissertation. The following sections describe the specific procedures undertaken in this multi-study dissertation to develop, pilot, and experimentally test the effects of the stereotype conditions (Study One) and representational mediator conditions (Study Two).

\section{Study One}

\section{Development of the Stereotype Conditions}

The focus of the pilot and main studies described in subsequent sections were 13 stereotype conditions that presumably described a target SAHM or WM. The target mother descriptions corresponded to the 11 most prevalent SAHM and WM stereotype profiles 
(Odenweller \& Rittenour, 2015, April), which include six SAHM stereotype profiles (i.e., busy and overworked; executive of the home; lazy, selfish, and lost; ideal mother; balancing work, family, and life; and non-traditional) and five WM stereotype profiles (i.e., overextended with no free time, hardworking and determined, supermom, flexible and family-oriented, and traditional). To exemplify the 11 stereotype profiles, the descriptions were developed using Odenweller and Rittenour's (2014, November) rich qualitative data. Two additional target mother descriptions were developed in this dissertation (i.e., a traditional SAHM and a nontraditional $\mathrm{WM}$ ) to explore stereotypes that are consistent with societal portrayals of these women (e.g., Brescoll \& Uhlmann, 2005; Bridges et al., 2002; Colaner \& Giles, 2008).

The final 13 target mother descriptions (see Appendix B) uniformly provided fictitious information about the target mother including her name (i.e., "Michelle"), number of children (i.e., two), and group membership (i.e., SAHM or WM). These details were included in each stereotype description for two reasons. First, "Michelle" was chosen as the target mothers' name because, according to Babycenter.com, "Michelle" was one of the top 10 baby names in 1980, the year in/around which the majority of participant mothers in the main study were expected to have been born. Additionally, the target mother was identified as a mother of two children because the average number of children per U.S. family has consistently been two since 1975 (Bureau, 2014). Given the potential similarity in participant and target mothers' ages and number of children, we expected participant mothers in the main study to categorize the target mother into their age and family size ingroups, generating more cognitive activity for the focus of this study: employment divisions. Second, control was imperative in the main study experiment. Thus, keeping the target mothers' name and number of children consistent across all conditions elicited greater confidence that the subsequent results were primarily due to 
participants' reactions to the stereotypes under study and not variations to a name or family size.

\section{Pilot Study}

A pilot study was conducted in order to investigate the qualitative distinctiveness across the stereotype conditions that would eventually appear as experimental manipulations in Study One's main study. Pilot participants were 219 male and female undergraduate students recruited from three Communication Studies courses at a large Mid-Atlantic university. After reading a brief cover letter and agreeing to participate in the pilot study, pilot participants completed one of 13 paper questionnaires during class time (see Appendix C). The questionnaires were nearly identical as they all included one forced-choice question and a series of demographic questions. The only variation in the questionnaires was the fictitious descriptions participants read about the target mothers (i.e., stereotype conditions). After reading the target mother description they were assigned, pilot participants completed one forced-choice question. Pilot participants were instructed to choose one of the provided descriptions (e.g., a traditional woman, a busy and overworked mother, an executive of the home) that best represents the target mother based on the description they read on the previous page. Pilot participants were encouraged to refrain from offering their personal opinion of the target mother and simply indicate how they thought she was described on the questionnaire. Pilot participants were permitted to refer back to the description before responding. After responding to the forced-choice question, pilot participants provided demographic information.

\section{Main Study}

Participants. A total of 654 mothers initially indicated interest in the study and clicked on the link provided in the email to begin the study. However, during data cleaning procedures, 125 of these mothers were removed because they did not complete a substantial amount of the 
online questionnaire or manifested as uni- or multi-variate outliers in the data set. Removing incomplete and outlier data resulted in a final sample of 529 participant mothers who were used in the subsequent analyses. More details about these data cleaning procedures can be found in the data analysis section of this chapter.

The final 529 participant mothers self-identified as stay-at-home (SAHM; $n=225$, $42.53 \%)$ and working $(\mathrm{WM} ; n=303,57.28 \%)$ mothers between the ages of 20 and $50(M=$ 33.11, $S D=4.60$ ) who had at least one child under 7 years old, with the majority of participant mothers $(n=421,79.58 \%)$ having two or three children. It was not necessary to impose specific definitions or criteria regarding mothers' identities because mothers' own self-categorization in the SAHM/WM social groups serve as optimal grounds for conducting a study on intergroup communication (Tajfel \& Turner, 1986). However, the restriction on children's ages was necessary to increase the salience of these mothers' group memberships and minimize complications associated with multiple group memberships (e.g., mothers who stay home initially and enter the workforce when their children begin kindergarten around age 6). Participant mothers were predominantly White $(n=495,93.57 \%)$, heterosexual $(n=510$, 96.41\%), married ( $n=492,93.01 \%)$, well-educated (Bachelor's degree: $n=193,36.48 \%$; graduate degree: $n=247,46.69 \%$ ), and affluent (annual household income of $\$ 80,000$ and above: $n=333,62.95 \%)$. One $(0.19 \%)$ participant mother did not report her ethnicity. One (0.19\%) participant mother did not report her sexual orientation. Six participant mothers $(1.13 \%)$ did not report their annual household income.

Although these demographic trends were generally consistent among participant SAHMs and WMs, several significant differences among these groups of mothers are worth noting. Participant SAHMs $(M=32.33, S D=4.84)$ were younger than participant WMs $(M=33.69, S D$ 
$=4.33 ; t[527]=3.41, p<.01)$ and reported having more children $(M=3.16, S D=1.18$, range $=$ $2-10)$ than participant WMs $(M=2.78, S D=.85$, range $=2-7 ; t[390.86]=-4.21, p<.001)$. A larger percentage of participant WMs reported holding graduate degrees $(n=180,59.21 \%)$ compared to participant SAHMs $\left(n=67,29.78 \% ; \chi^{2}[5, N=529]=47.78, p<.001\right)$. A larger percentage of participant WMs reported an annual household income over $\$ 100,000(n=160$, $52.63 \%)$ compared to participant SAHMs in this income bracket $\left(n=75,33.33 \% ; \chi^{2}[9, N=523]\right.$ $=31.86, p<.001$.

Two hundred and ninety-four participant WMs (96.71\%) indicated that they currently work for pay for an average of 37.13 hours per week $(S D=10.88$, range $=5-80$ hours per week), whereas 69 participant SAHMs (30.53\%) indicated that they currently work for pay for an average of 12.09 hours per week ( $S D=9.85$, range $=1-45$ hours per week), $t(360)=17.50, p=$ .001. The eight participant mothers (2.63\%) who identified as WMs but were not currently working indicated that they were on maternity leave but (all but one) plan to return to work, in a Ph.D. program, or unemployed but looking for work. Both participant SAHMs and WMs reported education $(n=122,23.06 \%)$, healthcare $(n=73,13.80 \%)$, and other $(n=70,13.23 \%)$ as the top three industries in which they work. A larger percentage of participant WMs reported working in the advertising/marketing/PR $(n=16,5.26 \%)$ and legal services industries $(n=12$, $3.95 \%)$, compared to participant SAHMs in the advertising/marketing/PR $(n=0,0 \%)$ and legal services $(n=5,2.22 \%)$ industries; whereas a larger percentage of participant SAHMs reported working in the child care $(n=18,8.00 \%)$ and administrative services $(n=10,4.44 \%)$ industries, compared to WMs in the child care $(n=6,1.97 \%)$ and administrative services $(n=7,2.30 \%)$ industries, $\chi^{2}(27, N=443)=56.39, p<.01$. Eighty-six $(16.26 \%)$ participant mothers did not report the industry in which they work. 
The majority of participant mothers $(n=420,79.40 \%)$ indicated that they had stayed home at some point since their first child was born $(M=1.53$ years, $S D=2.15$, range $=$ less than one month-18 years). Participant SAHMs reported staying home for longer durations of time ( $M$ $=3.18$ years, $S D=2.70$, range $=$ less than one month- 18 years), compared to participant $\mathrm{WMs}$ ' time at home ( $M=9$ months, $S D=1.13$, range = less than one month-13 years), $t(164.32)=-$ $10.42, p<.001$. Twelve $(2.27 \%)$ participant mothers—nine WMs and three SAHMs—indicated that they were on maternity leave when they participated in the study. Nine participant mothers on maternity leave (75.00\%)-eight WMs and one SAHM —indicated that they planned to return to work when their leave was over. The other three participant mothers on maternity leave $(25.00 \%)$ were unsure if they would return to work following their leave.

Procedure. Participant mothers were recruited via network, snowball, and convenience sampling techniques. The majority ( $n=340,64.27 \%$ ) of participant mothers were recruited via networking sampling from Facebook announcements in closed, private groups or public profile pages. Recruitment announcements were posted in the following closed Facebook groups: The New Mommy Club, Mother to Mother: Support \& Advice, Taking Mama Steps, Motherhood Support, Modern Mommy - (Northern) West Virginia, Mommy Connection, Mom Connection, Mothers Supporting Mothers, Pittsburgh Moms Club, Mommy Secrets Uncensored, Natural Mother Magazine Fan Forum, Mountain Mommas \& Poppas: WVU Working Parent Support Group, Sigma Delta Epsilon (Graduate Women in Science), Modern Mommy - The Mommy Files, From a Mother to a Mother, All Things Momma, Moms of Morgantown, I happy to be a mom - Columbus, From one Mother to Another (FOMA), Mothers \& Fathers - Bumps \& Babies, Mother's Group, WVU Alumni, Morgantown Breastfeeding Support Group, Formula Feeding Mommas, Evidence-based Crunchy Parenting, Black Mothers, Mon \& Preston County 
Rumor Mill, End the Mommy Wars, St. Francis Buddies, and Point Marion PA.

A smaller portion of participant mothers were recruited via snowball sampling from email and face-to-face referrals $(n=174,32.89 \%)$. To commence participant referrals, information was submitted to individuals who manage the following listservs and websites: General Electric Women's Network, formulamom.com, ctworkingmoms.com, WVU's Women and Gender's Studies Network, Working Mother Magazine, Motherlode Blogger, Mothering Magazine, motherhood.com, women.org, and Aquinas Academy of Pittsburgh. Additionally, information was emailed to mothers who, in the researcher's previous studies, indicated interest in future studies focused on families or motherhood. Referrals also originated from participant mothers.

The remaining participant mothers $(n=15,2.84 \%)$ were recruited via convenience sampling from undergraduate students in Communication Studies courses at a large Mid-Atlantic university. Students were given one extra credit point for each participant mother they recruited.

The recruitment scripts (see Appendix D) used across all sampling techniques were intentionally deceptive. Instead of advertising the "mommy wars" theme of this dissertation, mothers were solicited to participate in a 20-minute online study focused on mothers' adult friendships, communication, and wellbeing. Priming participant mothers about SAHMs' and WMs' tense relationships could have biased their responses as individuals prefer not to communicate prejudicial attitudes in public (Allport, 1954; Hecht, 1998b) and, thus, would likely respond in a more socially desirable (and inauthentic) manner. The recruitment scripts deceptively informed potential participant mothers that participation would involve: (1) completing a brief online questionnaire (comprised mostly of demographic information and their thoughts about motherhood) and (2) chatting with me and one other mother via a web/text-based 
chat technology (e.g., Google, Facebook, Windows Live, Yahoo). Participant mothers were told these online interactions with another participant mother (i.e., the target mother), with whom they would be randomly paired, would be brief, casual, and non-invasive. However, no participant mother-target mother interactions took place during this study. The interactions were only mentioned in the recruitment scripts to prime participant mothers to think about an upcoming contact experience with the target mother and elicit authentic and candid responses in the online questionnaire. Participant mothers were accurately informed that this study was part of the researcher's doctoral dissertation.

The recruitment script asked qualified and interested mothers to email the researcher within the week for more information about participating in this dissertation. The researcher responded to each participant mother within a few hours of receiving their emails and saved their contact information into a secure online database for later use. Within this first email (see Appendix E), participant mothers were alerted to the official launch of the study, which was scheduled for a week after the initial announcement on Facebook, and asked participant mothers for their patience while the researcher gathered information from other qualified and interested mothers. Delaying the launch (and setting a short deadline for completing the study upon launch) was necessary to minimize participants' communication outside the study and ensure the deception nature of the study was not shared. Due to staggered response rates from participant mothers, it took two weeks from the initial Facebook announcement to reach the proposed sample (i.e., 429).

On the designated day, a second email (see Appendix F) was distributed to all of the participant mothers who had expressed interest in this dissertation. In this email, participant mothers were provided a link to the online questionnaire (see Appendix G). Participant mothers 
believed the online questionnaire was to be completed before starting an online interaction with the target mother. Participant mothers were required to complete their participation within four days to be eligible for one of four $\$ 50$ Walmart gift cards.

After clicking on the link to the online questionnaire, participant mothers were directed to the consent form housed in qualtrics.com, the online survey software used to create the questionnaires. After reading the consent form and officially agreeing to participate in the study, participant mothers were randomly assigned to one of 13 experimental conditions and were immediately redirected to the online questionnaire that corresponded to their assigned condition. The online questionnaires were nearly identical across conditions as they all included demographic questions and the instruments described in the following section. The only variation in the online questionnaires was the fictitious target mother descriptions that participant mothers were asked to read (see the "development of stereotype conditions" section for more details about the target mother descriptions).

After reading the target mother descriptions, participant mothers responded to a series of questions about the target mother (i.e., the manipulation checks and instruments described in the following section). After completing all of the instruments, participant mothers were asked if they would like to advance to the online chat session or exit the study immediately. Regardless of their choice at this stage of the questionnaire, all participant mothers advanced to the same debriefing letter. In accordance with the West Virginia University's IRB deception debriefing protocol, the debriefing letter explained the intentions behind the deceptive study design and encouraged participant mothers to join a private Facebook group (i.e., Comma Mommas) that was created to facilitate connections among participant mothers outside of the study (see Appendix H). The debriefing letter also stressed the importance of not discussing details of the 
experiment with future participants until the close of the study was publically announced.

Instrumentation. The descriptive statistics (i.e., means, standard deviations, and Cronbach's alpha coefficients) for all of the instruments discussed in this section are available in Table 1. Unless otherwise noted, exploratory factor analyses (EFAs) revealed single-factor instruments based on criteria set forth by Costello and Osborne (2005) including eigenvalues of at least 1.0 , variances of $5.0 \%$ or above, primary/secondary loadings of $.50 / .30$ or $.60 / .40$. Before viewing the stereotype descriptions, participant mothers completed a series of demographic questions (e.g., age, ethnicity, paid work history, annual income), miscellaneous filler questions (to conceal the study's objective), and instruments that assessed ingroup identification and intergroup contact experience.

Ingroup identification. Ingroup identification was measured via two instruments. The first instrument, the Identity Fusion scale (Swann, Gomez, Seyle, Morales, \& Huici, 2009), is a single- item pictorial instrument that measures individuals' perceived identification with a particular person or group based on the degree to which two circles symmetrically overlap in a series of five Venn diagrams. Taking rigorous steps to establish the validity of their instrument, Swann et al. (2009) conducted five preliminary studies and three experiments to ensure that completion of this scale reflects a unique state of oneness that is distinct from non-identification or closeness. In the current study, the Identity Fusion scale measured participant mothers' identification with their SAHM/WM ingroup on a 5-point, Likert scale, with higher scores indicating greater identification with their ingroup.

The second instrument, the Ethnic Identity Scale (Umaña-Taylor, Yazedjian, \& BámacaGómez, 2004), is a 17-item instrument that measures the degree to which individuals have explored aspects related to their ethnicity (exploration), have resolved any issues related to their 


\section{Table 1.}

Descriptive and Correlation Statistics for Study One Variables

\begin{tabular}{|c|c|c|c|c|c|c|c|c|c|c|c|c|c|c|c|}
\hline Variables & $M$ & $S D$ & $\alpha$ & 1 & 2 & 3 & 4 & 5 & 6 & 7 & 8 & 9 & 10 & 11 & 12 \\
\hline 1. Ingroup Identity & 3.15 & 1.02 & -- & -- & & & & & & & & & & & \\
\hline 2. Mother Identity & 5.16 & .86 & .88 & $.39^{* *}$ & -- & & & & & & & & & & \\
\hline 3. Intergroup Contact - Quantity & 6.02 & .88 & .74 & $.13^{*}$ & $-.23^{* *}$ & -- & & & & & & & & & \\
\hline 4. Intergroup Contact - Quality & 5.45 & 1.04 & .86 & $-.17^{* *}$ & $-.18^{* *}$ & $.39^{* *}$ & -- & & & & & & & & \\
\hline 5. Feeling Thermometer & 6.70 & 2.84 & -- & -.01 & $.12^{*}$ & -.05 & $-.12^{* *}$ & -- & & & & & & & \\
\hline 6. Contempt ${ }^{\mathrm{a}}$ & 3.08 & 1.20 & .66 & .07 & -.01 & .05 & .08 & $-.66^{* *}$ & -- & & & & & & \\
\hline 7. Envy & 2.05 & 1.40 & .82 & -.07 & $-.17^{* *}$ & $.10^{*}$ & $.15^{* *}$ & -.01 & $-.13^{*}$ & -- & & & & & \\
\hline 8. Pity & 2.55 & 1.54 & .56 & -.03 & -.07 & -.05 & .05 & $-.23^{* *}$ & $.20^{* *}$ & -.07 & -- & & & & \\
\hline 9. Facilitative Behaviors ${ }^{\mathrm{b}}$ & 4.70 & 1.37 & .80 & .02 & .06 & -.10 & $-.10^{*}$ & $.51^{* *}$ & $-.52^{* *}$ & .04 & -.08 & -- & & & \\
\hline 10. Harmful Behaviors ${ }^{\mathrm{c}}$ & 1.43 & .65 & .58 & .02 & -.06 & .04 & $.15^{*}$ & $-.35^{* *}$ & $.32^{* *}$ & $.17^{* *}$ & $.19^{* *}$ & $-.41^{* *}$ & -- & & \\
\hline 11. Intergroup Anxiety & 3.24 & 1.11 & .86 & -.08 & $-.18^{* *}$ & .06 & $.20^{* *}$ & $-.56^{* *}$ & $.47^{* *}$ & $.25^{* *}$ & $.31^{* *}$ & $-.44^{* *}$ & $-.49^{* *}$ & -- & \\
\hline 12. Willingness to Communicate & 5.42 & 1.00 & .76 & .02 & -.06 & $.10^{*}$ & .07 & $-.27^{* *}$ & $.27^{* *}$ & -.05 & .05 & $-.28^{* *}$ & $.16^{* *}$ & $.24^{* *}$ & -- \\
\hline
\end{tabular}

Note. With the exception of the 5-point pictorial Identity Fusion scale and the 11-point pictorial Feeling Thermometer, all instruments were measured on a 7-point Likert scale.

${ }^{a}$ The contempt composite includes the two contempt and two reverse-coded admiration items.

${ }^{\mathrm{b}}$ The facilitative behaviors composite includes the two active and two passive facilitation items.

'The harmful behaviors composite includes the two active and two passive harm items.

$* .05<p>.01 * * p<.001$ 
ethnicity (resolution), and feel positively about their ethnicity (affirmation). Examples of the exploration items include: "I have attended events that have helped me learn more about my ethnicity" and "I have read books/magazines/newspapers or other materials that have taught me about my ethnicity". Examples of the resolution items include: "I am clear about what my ethnicity means to me" and "I understand how I feel about my ethnicity". Examples of the affirmation items include: "my feelings about my ethnicity are mostly negative" and "I wish I were of a different ethnicity". In this dissertation, the items were modified to represent the current focus on mother ingroups (e.g., "I am not happy with my group of SAHMs/WMs," "I feel negatively about my group of SAHMs/WMs," "I have learned about my group of SAHMs/WMs by doing things such as reading, search the Internet, or keeping up with current events"). To appropriately reflect modified focus of this instrument, this scale was renamed "mother identity" throughout this dissertation. Participant mothers rated each item on a 7-point, Likert scale ranging from not at all (1) to extremely (7). In accordance with previous researchers' procedures (e.g., Umaña-Taylor et al., 2004), participant mothers' scores were summed, with higher scores indicating stronger SAHM/WM ingroup identity. Researchers have previously obtained reliability coefficients ranging from .83 to .90 for the summed instrument (Brittian et al., 2015; Umaña-Taylor et al., 2004; Umaña-Taylor, Zeiders, \& Updegraff, 2013).

Intergroup contact. A two-part instrument of intergroup contact (Islam \& Hewstone, 1993) assessed participant mothers' contact experience with ingroup and outgroup mothers. Four items assessed quantitative aspects of mothers' intergroup contact experiences for both SAHM and WM targets (i.e., "I have close friends who are stay-at-home/working/non- mothers," "I speak to stay-at-home/working/non- mothers on a regular basis," "I have at least one acquaintance who is a stay-at-home/working/non- mother," "I have never talked to a stay-at- 
home/working/non- mother" [recoded]) on a 7-point, Likert scale ranging from strongly disagree (1) to strongly agree (7). Items that referenced neighbor and family member outgroup mothers (e.g., "I have neighbors who are stay-at-home/working/non- mothers," "I have family members who are stay-at-home/working/non- mothers") were replaced with items that referenced nonmothers to conceal the study's objectives. Items referencing contact with non-mothers were not used in subsequent analyses. Five 7-point, semantic differential items (e.g., voluntaryinvoluntary, pleasant-uncomfortable, competitive-cooperative) assessed qualitative elements of mothers' in- and outgroup contact experiences. Participant mothers' scores were summed along the quantitative and qualitative items for both targets, resulting in two, 9-item intergroup contact composites. Higher scores on the final two composites indicated more frequent and positive intergroup contact. Although Islam and Hewstone (1993) did not report the reliability of their instrument, Anderson, Harwood, and Hummert (2005) uncovered a reliability coefficient of .90 for this instrument.

After viewing the stereotype descriptions, participant mothers completed an experimental manipulation check and instruments that assessed outgroup attitudes, affective and behavioral responses, intergroup anxiety, and willingness to communicate.

Manipulation check. In order to validate the stereotype descriptions, a manipulation check was employed immediately after participant mothers read their assigned stereotype descriptions. In accordance with previous experimental research based on stereotypes (e.g., Ruble \& Zhang, 2012, November), the manipulation check included four multi-choice questions that asked participant mothers: (1) if they had carefully read the description about the target mother, (2) to which group the target mother belonged (i.e., SAHM or WM), (3) if they or anyone they knew had the same name as the target mother, and (4) which of the provided phrases 
(e.g., "an executive of her home," "a nontraditional woman," "an overextended mother with no free time") best described the target mother. These phrases aligned with the established stereotypes that were used to create the stereotype conditions. Thus, participant mothers assigned to one of the SAHM stereotype conditions chose from seven phrases (that corresponded to the seven SAHM stereotypes), while participant mothers assigned to one of the WM stereotype conditions chose from six phrases (that corresponded to the six WM stereotypes).

Outgroup attitudes. The Feeling Thermometer (Alwin, 1997) is a single item pictorial instrument that measures the intensity of individuals' attitudes toward a given group based on a "temperature reading" ranging from very cold and unfavorable (0) to very warm and favorable (10). On a 0-100 thermometer in increments of ten, participant mothers indicated how they felt about the target mother by choosing the "temperature reading" that best represented their attitudes. Higher scores, or temperature readings, indicated warmer and more favorable attitudes and lower scores, or temperature readings, indicated cold and unfavorable attitudes. The Feeling Thermometer is widely used to assess positive/negative outgroup attitudes (e.g., Brescoll \& Uhlmann, 2005; Soliz et al., 2010; R. N. Turner, Hewstone, Voci, \& Vonofakou, 2008).

Affective responses. Following the instructions, "To what extent did you feel the following emotions toward the other mother," a 10-item, 5-dimensional instrument measured participant mothers' affective responses to the target mother (Cuddy et al., 2007; Mackie, Devos, \& Smith, 2000): contempt (i.e., "contempt" and "disgust"), admiration (i.e., "admiration" and “pride”), pity (i.e., "pity” and "sympathy”), and envy (i.e., "envy” and "jealousy”). Participant mothers rated each item on a 7-point, Likert scale ranging from not at all (1) to extremely (7). Researchers have previously reported the following reliability ranges for each dimension: contempt $\alpha=.60-.77$, admiration $\alpha=.79-.80$, pity $\alpha=.71-.87$, and envy $\alpha=.82-.86$ 
(Cuddy et al., 2007).

Although Cuddy et al. (2007) summed participants' scores along the four dimensions to create four, 2-item affective composite variables, the items in this dissertation were summed differently to reduce the number of dependent variables in subsequent MANCOVAs. An EFA with maximum likelihood extraction and varimax orthogonal rotation, which extracted a threefactor solution, provided a sufficient argument for creating three affective composite variables. The "contempt," "disgust," "admiration" (recoded), and "pride" (recoded) comprised one factor that had an eigenvalue of 2.41 , primary loadings between .60 and .78 , and accounted for $30.06 \%$ of the instrument's total variance. The "envy" and "jealousy" items comprised another factor with an eigenvalue of 1.89 , primary loadings of .77, and accounted for $23.61 \%$ of the instrument's total variance. The "pity" and "sympathy" items comprised another factor with an eigenvalue of 1.28 , primary loadings of .72 and .85 , and accounted for $15.94 \%$ of the instrument's total variance. Higher scores on the three composite variables indicated stronger contempt (and weaker admiration), envy, and pity toward the target mother.

Behavioral responses. Following the instructions, "To what extent would you behave toward the other mother," a 14-item, six-dimensional instrument measured participant mothers' behavioral responses to the target mother (Cuddy et al., 2007): active facilitation (i.e., "help" and "protect"), active harm (i.e., "fight” and "attack"), passive facilitation (i.e., "cooperate with" and “associate with"), and passive harm (i.e., "exclude" and "demean”). Participant mothers rated each item on a 7-point, Likert scale ranging from not at all (1) to extremely (7). Researchers have previously reported the following reliability ranges for each dimension: active facilitation $\alpha$ $=.60-.91$, active harm $\alpha=.59-.86$, passive harm $\alpha=.68-.87$, and passive facilitation $\alpha=.61$ -.86 (Cuddy et al., 2007). 
Although Cuddy et al. (2007) summed participants' scores along the four dimensions to create four, 2-item behavioral composite variables, the items in this dissertation were summed differently to reduce the number of dependent variables in subsequent MANCOVAs. An EFA with maximum likelihood extraction and varimax orthogonal rotation, which extracted a twofactor solution, provided a sufficient argument for creating two behavioral composite variables. The four active and passive harm items comprised one factor with an eigenvalue of 3.14, primary loadings between .59 and .77 , and accounted for $39.28 \%$ of the instrument's total variance. The four active and passive facilitative items comprised another factor that had an eigenvalue of 1.74, primary loadings between .73 and .85 , and accounted for $21.77 \%$ of the instrument's total variance. Higher scores on the final two behavioral variables indicated stronger facilitative or harmful behaviors toward the target mother.

Intergroup anxiety. The 11-item Intergroup Anxiety Scale (Stephan \& Stephan, 1985) measured participant mothers' anxiety about interacting with the target mother. Following the instructions, “As I think about interacting with the other mother, I feel...," participant mothers indicated their level of agreement with each item (e.g., "awkward," "confident" [recoded], “irritated," "relaxed” [recoded], "happy" [recoded], "self-conscious") on a 7-point, Likert scale ranging from strongly disagree (1) to strongly agree (7). Participant mothers' scores on this instrument were summed, with higher scores indicating greater intergroup anxiety. Researchers have previously uncovered reliability coefficients ranging from .77 to .95 for various versions of this instrument (Barlow et al., 2009; Islam \& Hewstone, 1993; LaBelle, Booth-Butterfield, \& Rittenour, 2013; Soliz et al., 2010).

Willingness to communicate. Willingness to communicate was assessed in two ways. First, four items were designed specifically for this dissertation to measure participant mothers' 
willingness to communicate with the target mother. Participant mothers indicated their level of agreement with each statement (i.e., "I would like to participate in a program to help build connections between stay-at-home and working mothers," "I would be willing to speak to the other mother in an online chat session," "I would be willing to connect with the other mother on social media," and "I would be willing to meet the other mother face-to-face") on a 7-point, Likert scale ranging from strongly disagree (1) to strongly agree (7). These items were inspired by Ruble's (2011) 3-item assessment of American students' willingness to meet target Chinese international students, which obtained a reliability coefficient of .86. Participant mothers' scores on this instrument were summed, with higher scores will indicating greater willingness to communicate with the target mother.

Second, participant mothers' willingness to communicate with the target mother was also operationalized as advancement to the online interaction at the end of the questionnaire. Participant mothers were given a choice of interacting with the target mother (as originally advertised in the recruitment script) or exiting the study. Clicking the "schedule online chat session now" option indicated participant mothers' willingness to communicate with the target mother.

Data analysis. Before exploring the hypotheses in this study, the following four steps were conducted to ensure the data were complete and satisfied all univariate and multivariate assumptions. First, missing data were analyzed. A total of 654 mothers initially indicated interest in the study and clicked on the link provided in the second response email to begin participating. However, 104 of these mothers left a substantial portion of the online questionnaire blank (i.e., at least one whole instrument), with the majority $(n=99,95.19 \%)$ exiting the study early into the questionnaire. Although one mother $(0.96 \%)$ exited the study 
when prompted to describe herself to the target mother and five mothers $(4.81 \%)$ exited the study after reading the target mother description (i.e., stereotype condition), there did not appear to be any consistent trends among these six mothers' demographics (e.g., age, number of children, work status, marital status). Because analyzing this incomplete data would have been useless and uninformative, these cases were removed via listwise deletion.

After removing incomplete cases, missing data analyses were performed on the remaining data. Because missingness comprised only .18\% $-5.27 \%$ of each variable (and only $.55 \%$ of all values) and correlation results evidenced only three significant relationships between the dummy coded variables with missing data (e.g., facilitative behaviors, intergroup anxiety) and variables without missing data (e.g., willingness to communicate), missingness was considered random. Thus, with the exception of missing data on the Feeling Thermometer, missing data were imputed using the linear interpolation option in SPSS. The $12.40 \%$ of missing data on the Feeling Thermometer was attributed to the nature of the single-item, pictorial scale displayed to participant mothers on the online questionnaire (see Appendix I). Unbeknownst to the researchers until data collection had commenced, the Qualtrics version of the Feeling Thermometer automatically places the moveable pin at the " 5 " and participants must intentionally move the pin to select a final response even if their desired response is a " 5 ". Given that very few fives appeared in the original data set, it was reasoned that missing cases were the result of participant mothers thinking their responses of " 5 " (i.e., a neutral state of outgroup attitudes) had been saved on the questionnaire since that value initially appeared on the screen. Thus, missing data on this instrument were replaced with a " 5 ," which is 0.5 below the scale mean.

Second, the data were analyzed for uni- and multi-variate outliers. Twenty univariate 
outliers were detected from z-scores on intergroup contact, contempt, envy, harmful behaviors, and willingness to communicate 3.2 standard deviations above or below the mean. These outliers were removed because there did not appear to be any consistent trends among them. The data were also examined for multivariate outliers. One multivariate outlier was detected from a Mahalnobis Distance value above the chi-square/degrees of freedom critical value for eight dependent variables and a $p$ value of .001 (i.e., 26.12). This outlier was removed from the data set.

Third, the normality of the data was analyzed. Given that several of the variables were skewed based on their skewness/standard error ratio statistic falling above 3.2 or below -3.2 (i.e., contempt, envy, pity, harmful behaviors, quantity of previous intergroup contact, quality of previous intergroup contact, and willingness to communicate), square root and log transformations were conducted on these variables. The transformed variables were used in subsequent analyses; however, the mean and standard deviation reported is based on the raw, untransformed data.

Fourth, the data were analyzed for multicollinearity. A multiple regression in which the eight dependent variables from subsequent MANCOVAs predicted a random variable (i.e., participant code) was conducted. There was no evidence of multicollinearity as the tolerance values $(.48-.88)$ and VIF values $(1.13-2.10)$ were deemed acceptable (Tabachnick \& Fidell, 2013).

Finally, the bivariate associations among variables were analyzed. Correlational analyses revealed small to moderate relationships among many of the dependent variables $(r=.13-.66, p$ $<.001)$. However, pity and envy were not significantly correlated with either willingness to communicate or facilitative behaviors. Results of subsequent analyses should be interpreted with 
this assumption violation in mind.

Manipulation check. The qualitative distinctiveness of the stereotype conditions was tested via two chi-square analyses with the nominal level stereotype conditions and nominal level manipulation check (i.e., "based on the description you read about the other mother, which of the following options best describes her?"). Correct identification of the stereotype conditions was assessed via the chi-square value and cross tab percentages.

Hypothesis testing. The majority of hypotheses in Study One focused on differences between stereotype and group conditions. These hypotheses predicted that, when controlling for quantity and quality of previous intergroup contact, participant SAHMs' and WMs' outgroup attitudes (H1), affective (H4) and behavioral (H5) responses toward outgroup members, intergroup anxiety (H6), and willingness to communicate with outgroup members (H7) would differ across stereotype conditions.

After further consideration of these hypotheses following the prospectus meeting, two changes were made. First, ingroup identification was added as an additional control as this construct exerts powerful influence on intergroup outcomes (Allport, 1954; Tajfel \& Turner, 1986). Thus, two variables that assessed ingroup identification in this dissertation (i.e., ingroup identity and mother identity) were held constant as covariates in subsequent analyses along with quantity and quality of previous intergroup contact (which were included in the original hypotheses as control variables). Second, because the valence of the stereotype profiles were not firmly established (i.e., valence has only been uncovered for the individual stereotypes; Odenweller \& Rittenour, 2015, April), predictions regarding the causal relationships between positive/negative stereotypes and positive/negative intergroup communication outcomes were premature. Thus, the hypotheses were rewritten to emphasize an exploratory focus on the 
various effects of the stereotype conditions.

To parsimoniously analyze between-group differences as done in the pilot study, SAHM stereotype conditions and WM stereotype conditions were separated and analyzed in two different MANCOVAs. MANCOVA was chosen because this analysis maximizes power when examining multiple outcomes that are moderately correlated and holding other variables constant (Tabachnick \& Fidell, 2013). One 7 (SAHM stereotype conditions: busy and overworked; executive of the home; lazy, selfish, and lost; ideal mother; balancing work, family, and life; non-traditional; and traditional) x 2 (participant ingroup membership: SAHM and WM) betweensubjects factorial MANCOVA and one 6 (WM stereotype conditions: overextended with no free time, hardworking and determined, supermom, flexible and family-oriented, non-traditional, and traditional) x 2 (participant ingroup membership: SAHM and WM) between-subjects factorial MANCOVA were conducted for the eight dependent variables (i.e., outgroup attitudes; contempt, pity, and envy affective responses, facilitative and harmful behavioral responses, intergroup anxiety, and willingness to communicate) with four covariates (i.e., quantity of previous intergroup contact, quality of previous intergroup contact, ingroup identity, and mother identity).

Three possible outcomes exist for this MANCOVA. Significant omnibus multivariate tests (i.e., Wilks' Lambda) for stereotype conditions would indicate significant differences between stereotypes descriptions in terms of intergroup communication outcomes (e.g., the supermom stereotype condition elicits more favorable outgroup attitudes, feelings, and behaviors compared to the traditional stereotype condition). Univariate main effects will be examined to determine which dependent variables are significantly different across the stereotype conditions and, thus, which hypotheses (i.e., H1, H4, H5, H6, or H7) are supported. Significant main 
effects at the univariate level for stereotype conditions would suggest stereotype descriptions, regardless of participant mothers' ingroup membership, influence participant mothers' levels of the dependent variables. Due to the exploratory nature of this study, post hoc Bonferroni corrections would determine which stereotype descriptions were significantly different from each other in regard to the dependent variables that obtain significance at the univariate level. The results of these post-hoc analyses, which are more appropriate than contrasts for this exploratory study in which favorability of stereotype conditions has yet to be determined, will illuminate which stereotype descriptions produce more (un)favorable intergroup communication outcomes.

Significant omnibus multivariate tests (i.e., Wilks’ Lambda) for group conditions would indicate significant differences between participant mothers' ingroup membership in terms of intergroup communication outcomes (e.g., SAHMs, compared to WMs, report more favorable attitudes, feeling, or behaviors when the target mother is a SAHM). Univariate main effects will be examined to determine which dependent variables are significantly different across the group conditions. Significant main effects for group conditions would suggest participant mothers' ingroup membership, regardless of stereotype descriptions, influences participant mothers' levels of dependent variables.

Significant omnibus multivariate tests (i.e., Wilks’ Lambda) for the stereotype x group interaction would indicate that differences in the levels of intergroup communication outcomes are influenced by a complex combination of stereotype description and participant mothers' ingroup membership (e.g., SAHMs, compared to WMs, report more favorable attitudes, feelings, or behaviors for the ideal mother SAHM stereotype but more unfavorable attitudes, feelings, or behaviors for the lazy, selfish, and lost SAHM stereotype). The stereotype x group interactions will be examined at the univariate level to determine which dependent variables are influenced 
by the interaction; thus, qualifying the main effects discussed previously. Significant stereotype $\mathrm{x}$ group interactions would demonstrate ingroup favoritism (or outgroup bias) based on the specific stereotype descriptions.

In addition to the continuous measure of willingness to communicate included in the MANCOVA, participant mothers' willingness to communicate was operationalized as advancement to the online interaction. A chi-square analysis with the nominal level stereotype conditions, nominal level group condition (i.e., participant mothers' ingroup membership), and nominal level advancement (i.e., clicking the "schedule online chat session now" option on the questionnaire). A significant chi-square would suggest a relationship between exiting the study, stereotype condition, and participant mothers' ingroup membership.

Two additional hypotheses examined ingroup favoritism. H2 predicted that participant SAHMs and WMs would report more positive attitudes when the target mother was a member of the participant mothers' ingroup compared to when the target mother was a member of the participant mothers' outgroup. Two one-tailed, independent samples $t$-test were employed to, first, compare SAHMs' and WMs' mean scores on the Feeling Thermometer (Alwin, 1997) when SAHMs are the target and compare WMs' and SAHMs' mean scores on the Feeling Thermometer (Alwin, 1997) when WMs are the target. The data file was split to focus the analyses on either SAHM stereotype conditions or WM stereotype conditions. Significant $t$-test results would demonstrate that ingroup membership influences more favorable ingroup attitudes (i.e., ingroup favoritism).

H3 predicted that participant SAHMs would report more positive attitudes for target SAHMs compared to participant WMs' attitudes for target working mothers. A one-tailed, independent samples $t$-test was employed to compare mean scores on the Feeling Thermometer 
(Alwin, 1997) for the two ingroup conditions (i.e., SAHMs assigned to SAHM stereotype conditions and WMs assigned to WM stereotype conditions). Significant $t$-test results would indicate participant SAHMs' attitudes toward target SAHMs are more favorable than WMs' attitudes toward target WMs; thus, demonstrating SAHMs' ingroup favoritism is stronger than WMs' ingroup favoritism.

\section{Study Two}

\section{Development of Representational Mediator Conditions}

The focus of the pilot and main studies described in subsequent sections were four introduction messages presumably sent from a target SAHM or WM. The messages were designed specifically for this study based on previous CIIM research (e.g., Dovidio et al., 1998; Gaertner et al., 1999; Gaertner et al., 1990), focused on the topic of maintaining adult friendships after becoming a mother, and communicated the CIIM's (Gaertner et al., 1994) four representational mediators: (1) one group recategorization (e.g., references to "mothers" only), (2) two subgroups in one group recategorization (e.g., references to "SAHMs/WMs" and "mothers"), (3) two groups categorization (e.g., references to "SAHMs/WMs" only), and (4) separate individuals decategorization (e.g., references to "you" and "me" as people). The message topic was inspired by mothers' responses to an open-ended question in a previous research study (Odenweller \& Rittenour, 2014, November), which revealed "maintaining adult relationships" as a salient discussion topic in mother-mother interactions. Representational mediator conditions also referenced fictitious information about the target mother including her name (i.e., "Michelle"), number of children (i.e., two), and group membership (i.e., SAHM or WM). The target mothers' group membership was intentionally selected to be different from participant mother's self-identified ingroup in order to prime ingroup/outgroup distinctions. As 
in Study One, the target mother in each representational mediator condition was named "Michelle" in order to ensure participant mothers' were focused on the SAHM/WM categories and to assert subsequent results were primarily due to participant mothers' reactions to the mediators under study. See Appendix $\mathrm{J}$ for the four representational mediator conditions provided to participants as messages from the target mother.

\section{Pilot Study}

A pilot study was conducted in order to investigate the qualitative distinctiveness across the representational mediator conditions that would eventually appear as experimental manipulations in Study Two's main study. Pilot participants were 13 male and female undergraduate students recruited via one Communication Studies course and 32 mothers with at least one child over the age of six recruited via network sampling. The demographic criteria were selected for pilot mothers to ensure they would not eventually become participants in the main study (see inclusion criteria below). After reading a brief cover letter and agreeing to participate in the study, pilot participants completed one of four paper (undergraduate students) or online (mothers) questionnaires (see Appendix K). The questionnaires were nearly identical as they all included demographic questions and one forced-choice question. The only variation in the questionnaires was the message, ostensibly sent from a SAHM to a WM, that pilot participants were asked to read (i.e., representational mediator conditions). After reading the SAHM's message they were assigned, pilot participants completed one forced-choice question. Pilot participants were instructed to choose one of the provided descriptions (i.e., members of one, large group of mothers; members of two, smaller subgroups of stay-at-home and working mothers that fall under one larger group of mothers; members of two completely separate groups of stay-at-home and working mothers; unique and separate individuals) that best represents how 
a WM would feel after reading the SAHM's message. Pilot participants were permitted to refer back to the message before responding. After responding to the forced-choice question, pilot participants provided demographic information.

\section{Main Study}

Participants. A total of 196 mothers initially indicated interest in the study and clicked on the link provided in the second response email to begin the study. However, during data cleaning procedures, 42 of these mothers were removed because they did not complete a substantial amount of the online questionnaire or manifested as a univariate outlier in the data set. Removing incomplete and outlier data resulted in a final sample of 154 participant mothers who were used in the subsequent analyses. More details about these data cleaning procedures can be found in the data analysis section of this chapter.

The final 154 participant mothers self-identified as stay-at-home (SAHM; $n=70$, $45.45 \%)$ and working (WM; $n=84,54.55 \%)$ mothers between the ages of 20 and $50(M=33.72$, $S D=5.27)$ who had at least one child under 7 years old, with the majority of participant mothers ( $n=421,79.58 \%$ ) having two or three children. As mentioned in Study One, although mothers' self-categorization in the WM/SAHM groups was sufficient to induce intergroup outcomes, restricting children's ages was necessary to increase the salience of these mothers' group memberships and minimize complications associated with non-exclusive "working/staying home" group memberships. Participant mothers were predominantly White $(n=144,93.51 \%)$, heterosexual $(n=144,93.51 \%)$, married $(n=144,93.51 \%)$, well-educated (Bachelor's degree: $n$ $=53,34.42 \%$; graduate degree: $n=66,42.86 \%$ ), and affluent (annual household income of $\$ 80,000$ and above: $n=85,55.19 \%)$. One $(0.65 \%)$ participant mother did not report her annual household income. 
Although these demographic trends were generally consistent across participant SAHMs and WMs, several significant differences among these groups of mothers are worth noting. Participant SAHMs $(M=32.39, S D=5.65$, range $20-50)$ were younger than participant WMs $(M=34.83, S D=4.68$, range $24-46 ; t[152]=2.94, p<.01)$ and reported having more children $(M=2.39, S D=1.58$, range $=1-9)$ than participant WMs $(M=1.85, S D=1.00$, range $=1-6 ;$ $t[112.27]=-2.48, p<.05)$. A larger percentage of participant WMs reported holding graduate degrees $(n=50,59.52 \%)$ compared to participant SAHMs $\left(n=16,22.86 \% ; \chi^{2}[6, N=154]=\right.$ 25.07, $p<.001)$. A larger percentage of participant WMs reported an annual household income over $\$ 100,000(n=42,50.00 \%)$ compared to participant SAHMs in this income bracket $(n=17$, $\left.24.29 \% ; \chi^{2}[9, N=153]=20.33, p<.05\right)$.

Seventy-nine participant WMs (94.05\%) indicated that they currently work for pay for an average of 38.04 hours per week $(S D=9.27$, range $=10-60$ hours per week), whereas 16 participant SAHMs (22.86\%) indicated that they currently work for pay for an average of 15.25 hours per week $(S D=14.17$, range $=2-45$ hours per week; $t[17.69]=6.17, p<.001)$. Both participant WMs and SAHMs reported education $(n=34,22.08 \%)$, healthcare $(n=19,12.34 \%)$, and other $(n=25,16.23 \%)$ as the top three industries in which they work. However, a larger percentage of participant WMs reported working in the advertising/marketing/PR industry $(n=$ $6,7.14 \%)$ compared to participant SAHMs in this industry $(n=2,2.86 \%)$; whereas a larger percentage of participant SAHMs reported working in the child care industry $(n=5,7.14 \%)$, compared to WMs in this industry $\left(n=0,0 \% ; \chi^{2}[19, N=154]=65.33, p<.001\right)$. Thirty $(19.48 \%)$ participant mothers did not report the industry in which they work.

The majority of participant mothers $(n=118,76.62 \%)$ indicated that they had stayed home at some point since their first child was born $(M=1.87$ years, $S D=3.11$, range $=$ less than 
one month-20 years). Participant SAHMs reported staying home for longer durations of time ( $M$ $=3.35$ years, $S D=3.53$, range $=$ less than one month-15 years), compared to participant WMs' time at home $(M=1.05$ years, $S D=2.52$, range = less than one month-20 years; $t[64.44]=-$ $3.72, p<.001)$. Two $(1.30 \%)$ participant WMs indicated that they were on maternity leave when they participated in the study, and all of them (100\%) indicated that they planned to return to work when their leave was over.

Procedure. Participant mothers were recruited via network, snowball, and convenience sampling techniques. The majority $(n=109,70.78 \%)$ of participant mothers were recruited via networking sampling from Facebook announcements in closed, private groups or public profile pages. Recruitment announcements for Study Two were posted in the same Facebook groups listed in Study One. A smaller portion of participant mothers were recruited via snowball sampling from email and face-to-face referrals $(n=41,26.62 \%)$. As described in Study One, the referral process was initiated by submitting information to listserv and website managers and mothers who participated in the researcher's previous studies. Referrals also originated from participant mothers. The remaining mothers $(n=4,2.60 \%)$ were recruited via convenience sampling from undergraduate students in Communication Studies courses at a large Mid-Atlantic university. Students were given one extra credit point for each participant mother they recruited.

Recruitment for Study Two followed the same procedures, and occurred simultaneously, as the procedures outlined in Study One. The deceptive recruitment scripts (see Appendix D), which were used to conceal the objectives of the study and prevent biased responses, informed potential participant mothers that participation would involve a brief online questionnaire and casual, non-invasive online interactions with target mothers with whom they would be randomly paired. As in Study One, no participant mother-target mother interactions took place during this 
study. Mention of the online interactions was only intended to prime participant mothers to think about contact with the target mother and elicit their authentic and candid responses about the target mother.

Similar to the recruitment scripts in Study One, qualified and interested mothers were instructed to email the researcher for more information about participating in this dissertation. The researcher responded to participant mothers via two emails over a two-week period as she attempted to reach the proposed sample (i.e., 180). In the first email (see Appendix E), participant mothers were informed of the study's forthcoming launch. In this email, participant mothers were provided a link to the online questionnaire (see Appendix L). Participant mothers believed the online questionnaire was to be completed before starting an online interaction with the target mother. Participant mothers were required to complete their participation within four days of receiving my second email to be eligible for one of four $\$ 50$ Walmart gift cards.

After clicking on the link to the online questionnaire, participant mothers were directed to the consent form housed in qualtrics.com. After providing consent, participant mothers were randomly assigned to one of four experimental conditions and were immediately redirected to the online questionnaire that corresponded to their assigned condition. The online questionnaires were nearly identical across conditions as they all included demographic questions and the instruments described in the following section. The only variation in the online questionnaires was the introduction messages from fictitious target mothers that participant mothers were asked to read (see the "development of representational mediator conditions" section for more details about the target mother introduction messages).

After reading the message, participant mothers were asked to respond to a series of questions about the target mother (i.e., the manipulation checks and instruments described in the 
following section). After completing all of the instruments, participant mothers were asked if they would like to advance to the online chat session or exit the study immediately. Regardless of their choice at this stage of the questionnaire, all participant mothers advanced to the same debriefing letter. The debriefing procedures at this phase in Study Two mirror those outlined in Study One.

Instrumentation. The descriptive statistics (i.e., means, standard deviations, and Cronbach's alpha coefficients) for all of the instruments discussed in this section are available in Table 2. Unless otherwise noted, exploratory factor analyses (EFAs) revealed single-factor instruments based on criteria set forth by Costello and Osborne (2005) including eigenvalues of at least 1.0 , variances of $5.0 \%$ or above, primary/secondary loadings of $.50 / .30$ or $.60 / .40$. Before viewing the target mother's introduction message, participant mothers completed a series of demographic questions (e.g., age, ethnicity, paid work history, annual income), miscellaneous filler questions (to conceal the study's objective), and instruments that assessed ingroup identification and intergroup contact experience.

Ingroup identification. Ingroup identification was measured via two instruments. The first instrument, the Identity Fusion scale (Swann, Gomez, Seyle, Morales, \& Huici, 2009), is a single item pictorial instrument that measures individuals' perceived identification with a particular person or group based on the degree to which two circles symmetrically overlap in a series of five Venn diagrams. Taking rigorous steps to establish the validity of their instrument, Swann et al. (2009) conducted five preliminary studies and three experiments to ensure that completion of this scale reflects a unique state of oneness that is distinct from non-identification or closeness. In the current study, the Identity Fusion scale measured participant mothers' identification with their SAHM/WM ingroup on a 5-point, Likert scale, with higher scores 
Table 2.

Descriptive and Correlation Statistics for Study Two Variables

\begin{tabular}{|c|c|c|c|c|c|c|c|c|c|c|c|c|}
\hline Variables & $M$ & $S D$ & $\alpha$ & 1 & 2 & 3 & 4 & 5 & 6 & 7 & 8 & 9 \\
\hline 1. Ingroup Identity & 3.03 & 1.02 & -- & -- & & & & & & & & \\
\hline 2. Mother Identity & 5.19 & .82 & .86 & $.35^{* *}$ & -- & & & & & & & \\
\hline 3. Intergroup Contact - Quantity & 4.78 & .53 & .63 & -.08 & $-.23^{*}$ & -- & & & & & & \\
\hline 4. Intergroup Contact - Quality & 5.32 & .98 & .85 & $-.22^{*}$ & -.16 & $.27^{*}$ & -- & & & & & \\
\hline 5. Stereotype Reliance & 4.00 & .54 & .55 & $.22^{*}$ & .14 & $-.17^{*}$ & $-.19^{*}$ & -- & & & & \\
\hline 6. Feeling Thermometer & 8.13 & 2.27 & -- & .06 & $.22^{*}$ & $-.21^{*}$ & $-.24^{*}$ & $.25^{*}$ & -- & & & \\
\hline 7. Outgroup Typicality & 4.44 & .76 & .61 & -.02 & -.02 & .03 & .08 & -.07 & -.08 & -- & & \\
\hline 8. Helping & 2.63 & 2.48 & -- & .15 & .12 & -.10 & .05 & -.02 & .02 & .01 & -- & \\
\hline 9. Willingness to Communicate & 5.22 & 1.16 & .85 & -.03 & -.10 & .14 & .15 & $-.20^{*}$ & $-.36^{* *}$ & -.01 & $-.22^{*}$ & -- \\
\hline
\end{tabular}

Note. All instruments were measured on a 7-point Likert scale, with the exception of the 5-point, one pictorial item for the Identity Fusion scale; the 11-point, one pictorial item for the Feeling Thermometer; and helping assessed by time spent (in minutes) responding to the one open-ended question.

$* .05<p>.01 * * p<.001$ 
indicating greater identification with their ingroup.

The second instrument, the Ethnic Identity Scale (Umaña-Taylor et al., 2004), is a 17item instrument that measures the degree to which individuals have explored aspects related to their ethnicity (exploration), have resolved any issues related to their ethnicity (resolution), and feel positively about their ethnicity (affirmation). Examples of the exploration items include: "I have attended events that have helped me learn more about my ethnicity" and "I have read books/magazines/newspapers or other materials that have taught me about my ethnicity." Examples of the resolution items include: "I am clear about what my ethnicity means to me" and "I understand how I feel about my ethnicity". Examples of the affirmation items include: "my feelings about my ethnicity are mostly negative" and "I wish I were of a different ethnicity". In this dissertation, the items were modified to represent the current focus on mother ingroups (e.g., "I am not happy with my group of stay-at-home/working mothers" "I feel negatively about my group of stay-at-home/working mothers," "I have learned about my group of stay-athome/working mothers by doing things such as reading, search the Internet, or keeping up with current events"). To appropriately reflect modified focus of this instrument, this scale was renamed "mother identity" throughout this dissertation. Participant mothers rated each item on a 7-point, Likert scale ranging from not at all (1) to extremely (7). In accordance with previous researchers' procedures (e.g., Umaña-Taylor et al., 2004), participant mothers' scores were summed, with higher scores indicating stronger SAHM/WM ingroup identity. Researchers have previously obtained reliability coefficients ranging from .83 to .90 for the summed instrument (Brittian et al., 2015; Umaña-Taylor et al., 2004; Umaña-Taylor et al., 2013).

Intergroup contact. A two-part instrument of intergroup contact (Islam \& Hewstone, 1993) assessed participant mothers' contact experience with ingroup and outgroup mothers. 
Four items assessed quantitative aspects of mothers' intergroup contact experiences for both SAHM and WM targets (i.e., "I have close friends who are stay-at-home/working/non- mothers," "I speak to stay-at-home/working/non- mothers on a regular basis," "I have at least one acquaintance who is a stay-at-home/working/non-mother," "I have never talked to a stay-athome/working/non- mother" [recoded]) on a 7-point, Likert scale ranging from strongly disagree (1) to strongly agree (7). Items that referenced neighbor and family member outgroup mothers (e.g., "I have neighbors who are stay-at-home/working/non- mothers," "I have family members who are stay-at-home/working/non- mothers") were replaced with items that referenced nonmothers to conceal the study's objectives. Items referencing contact with non-mothers were not used in subsequent analyses. Five 7-point, semantic differential items (e.g., voluntaryinvoluntary, pleasant-uncomfortable, competitive-cooperative) assessed qualitative elements of mothers' in- and outgroup contact experiences. Participant mothers' scores were summed along the quantitative and qualitative items for both targets, resulting in two, 9-item intergroup contact composites. Higher scores on the final two composites indicated more frequent and positive intergroup contact. Although Islam and Hewstone (1993) did not report the reliability of their instrument, Anderson, Harwood, and Hummert (2005) uncovered a reliability coefficient of .90 for this instrument.

After viewing the target mother's introduction message, participant mothers completed an experimental manipulation check and instruments that assessed stereotype reliance, outgroup attitudes, outgroup typicality, helping, and willingness to communicate.

Manipulation check. In order to validate the categorization elicited in each target mother's message, a manipulation check was employed immediately after participant mothers read their assigned message. In accordance with previous experimental research based on the 
CIIM (e.g., Dovidio et al., 1998; Gaertner et al., 1999; Gaertner et al., 1989; Gaertner et al., 1990), the manipulation check included four multiple-choice questions that asked participant mothers: (1) if they had carefully read the message from the target mother, (2) to which group the target mother belonged (i.e., SAHM or WM), (3) if they or anyone they knew had the same name as the target mother, and (4) which of the provided phrases (i.e., "members of two completely separate groups of stay-at-home and working mothers;" "members of one large group of mothers;" "members of two, smaller subgroups of stay-at-home and working mothers that fall under one larger group of mothers;" or "unique and separate individuals") best described how they felt after reading the target mother's message. These phrases aligned with the four representational mediators in the CIIM that were used to create the message conditions.

Stereotype reliance. The instrument designed to measure participant mothers' stereotype reliance in this dissertation was inspired by Owens and Massey's (ADD 2011) three-item, semantic differential measure of racial stereotype threat (e.g., lazy-hardworking, intelligentunintelligent, give up easily-stick with it) and the most prevalent SAHM/WM stereotype profiles (Odenweller \& Rittenour, 2015, April). The SAHM/WM stereotype profiles were converted into an 11-item, 7-point Likert scale ranging from strongly disagree (1) to strongly agree (7). A Likert scale was preferred over semantic differential items in this dissertation because creating a continuum with dichotomized versions of some of the stereotypes did not make conceptual sense (e.g., supermom, executive of the home, helicopter parent). Participant mothers indicated their level of agreement regarding each stereotype's applicability to the target mother. Although Owens and Massey (2011) did not report the reliability of their three-item measure of stereotype threat, they performed rigorous factor and structural equation analyses to demonstrate the psychometric soundness of their instrument. Participant mothers' scores on this instrument were 
summed, with higher scores indicating a stronger reliance on stereotypes.

Outgroup attitudes. The Feeling Thermometer (Alwin, 1997) is a single item pictorial instrument that measures the intensity of individuals' attitudes toward a given group based on a "temperature reading" ranging from very cold and unfavorable (0) to very warm and favorable (10). On a 0-100 thermometer in increments of ten, participant mothers indicated how they felt about the target mother by choosing the "temperature reading" that best represented their attitudes. Higher scores, or temperature readings, indicated warmer and more favorable attitudes and lower scores, or temperature readings, indicated cold and unfavorable attitudes. The Feeling Thermometer is widely used to assess positive/negative outgroup attitudes (e.g., Brescoll \& Uhlmann, 2005; Soliz et al., 2010; R. N. Turner et al., 2008).

Outgroup typicality. Three items measured participant mothers' perceptions of the target mother's typicality, or how well the target mother represents their (out)group as a whole. These items were inspired by measures used in previous research on group salience and typicality (e.g., Brown et al., 1999; L. Johnston \& Hewstone, 1992). Participant mothers indicated their level of agreement with each statement (i.e., "The other mother is similar to other stay-at-home/working mothers," "The other mother is a typical stay-at-home/working mother," "The other mother is different from other stay-at-home/working mothers") on a 7-point, Likert scale ranging from strongly disagree (1) to strongly agree (7). Participant mothers' scores on this instrument were summed, with higher scores indicating stronger perceptions of outgroup typicality for the target mother. Researchers have previously obtained reliability coefficients ranging from .63 to .90 for similar versions of this instrument (Hains, Hogg, \& Duck, 1997; Harwood, Raman, \& Hewstone, 2006; Jetten, Spears, \& Antony S. R. Manstead, 1997; Wang, Walther, \& Hancock, 2009).

Helping. Drawing upon Dovidio et al.'s (1997) measure of helping, participant mothers' 
helping was assessed through a real-life scenario that evokes quantifiable helping behaviors. In this scenario, participant mothers were asked to list tangible ideas, resources they can access (e.g., websites, social media), or even specific names and contact information the target mother can use to find a babysitter for a night out. Helping was operationalized as the duration of time spent on this question; thus, the more time participant mothers devoted to answering this question (measured in minutes), the more help they offered the target mother.

Willingness to communicate. Four items were designed specifically for this dissertation to measure participant mothers' willingness to communicate with the target mother. Participant mothers indicated their level of agreement with each statement (i.e., "I would like to participate in a program to help build connections between stay-at-home and working mothers," "I would be willing to speak to the other mother in an online chat session," "I would be willing to connect with the other mother on social media," and "I would be willing to meet the other mother faceto-face") on a 7-point, Likert scale ranging from strongly disagree (1) to strongly agree (7). These items were inspired by Ruble's (2011) 3-item assessment of American students' willingness to meet target Chinese international students, which obtained a reliability coefficient of .86. Participant mothers' scores on this instrument were summed, with higher scores will indicating greater willingness to communicate with the target mother.

Data analysis. Before exploring the hypotheses in this study, the following four steps were conducted to ensure the data were complete and satisfied all univariate and multivariate assumptions. First, missing data were analyzed. A total of 196 mothers initially indicated interest in the study and clicked on the link provided in the email to begin participating. However, 37 of these mothers left a substantial portion of the online questionnaire blank (i.e., at least one whole instrument), with the majority $(n=36,97.30 \%)$ exiting the study early into the 
questionnaire. One mother $(2.70 \%)$ exited the study after reading the target mother introduction message (i.e., representational mediator condition). Because analyzing this incomplete data would have been useless and uninformative, these cases were removed via listwise deletion.

After removing incomplete cases, missing data analyses were performed on the remaining data. Because missingness comprised only $1.26 \%-6.29 \%$ of each variable (and only $2.52 \%$ of all values) and correlation results evidenced no significant relationships between the dummy coded variables with missing data and variables without missing data, missingness was considered random. Thus, with the exception of missing data on the Feeling Thermometer, missing data were imputed using the linear interpolation option in SPSS. The $10.69 \%$ of missing data on the Feeling Thermometer was replaced with a "5," which is 0.5 below the scale mean. As described in Study One, missingness on this instrument was attributed to the nature of the single-item, pictorial scale displayed to participant mothers on the online questionnaire (see Appendix I).

Second, the data were analyzed for univariate and multivariate outliers. Five univariate outliers were detected from z-scores on quality of intergroup contact, stereotype reliance, outgroup typicality, and helping falling 3.2 standard deviations above or below the mean. These outliers were removed because there did not appear to be any consistent demographic trends among them. The data set was also examined for multivariate outliers. No multivariate outliers were detected as all Mahalnobis Distance values fell below the chi-square/degrees of freedom critical value for five dependent variables and a $p$ value of .001 (i.e., 20.52).

Third, the normality of the data was analyzed. Given that several of the variables were skewed based on their skewness/standard error ratio statistic falling above 3.2 or below -3.2 (i.e., outgroup typicality, helping, and willingness to communicate), square root and log 
transformations were conducted on these variables. The transformed variables were used in subsequent analyses; however, the mean and standard deviation reported is based on the raw, untransformed data.

Fourth, the data were analyzed for multicollinearity. A multiple regression in which the five dependent variables from subsequent MANCOVAs predicted a random variable (i.e., participant code) was conducted. There was no evidence of multicollinearity as the tolerance values $(.80-.99)$ and VIF (1.01 - 1.26) values were deemed acceptable (Tabachnick \& Fidell, 2013).

Finally, the bivariate associations among variables were analyzed. Correlational analyses revealed small to moderate relationships among many of the variables $(r=-.20--.37, p<.01)$. However, outgroup typicality was not significantly correlated with the other dependent variables. Results of subsequent analyses should be interpreted with this assumption violation in mind.

Manipulation check. The qualitative distinctiveness of the representational mediator conditions was tested via two chi-square analyses with the nominal level stereotype conditions and nominal level manipulation check (i.e., "after reading the other mother's message, I feel as if we are...?”). Correct identification of the message conditions was assessed via the chi-square value and cross tab percentages.

Hypothesis testing. The four hypotheses in Study Two focused on differences between the four representational mediators. These hypotheses predicted that, following an intergroup contact experience, participant SAHMs' and WMs' reliance on stereotypes (H8), outgroup attitudes (H9), outgroup typicality (H10), helping (H11), and willingness to communicate with outgroup members (H12) would differ across representational mediator conditions put forth in the common in-group identity model. Specifically, representational mediator conditions that 
elicited one group recategorization, two subgroup in one group recategorization, and unique separate individuals decategorization were predicted to draw upon fewer stereotypes, elicit more favorable outgroup attitudes, elicit stronger perceptions of outgroup typicality, elicit more helping behaviors, and elicit a greater willingness to communicate; whereas the representational mediator condition that elicited two group categorization was predicted to draw upon more stereotypes, elicit less favorable outgroup attitudes, elicit weaker perceptions of outgroup typicality, elicit fewer helping behaviors, and elicit less willingness to communicate. After further consideration of these hypotheses following the prospectus meeting, previous intergroup contact experience and ingroup identification were included as controls as these constructs alone exert powerful influence on the categorization process and intergroup outcomes (Allport, 1954; Islam \& Hewstone, 1993; Tajfel \& Turner, 1986). Thus, the four variables that assessed these constructs in this dissertation (i.e., quantity of intergroup contact, quality of intergroup contact, ingroup identity, and mother identity) were held constant as covariates in subsequent analyses.

The hypotheses were analyzed via one MANCOVA. MANCOVA was chosen because this analysis maximizes power when examining multiple outcomes that are moderately correlated and holding other variables constant (Tabachnick \& Fidell, 2013). A one-way between-subjects MANCOVA was conducted for the five dependent variables (i.e., stereotype reliance, outgroup attitudes, outgroup typicality, helping, willingness to communicate) with four covariates (i.e., quantity of previous intergroup contact, quality previous intergroup contact, ingroup identification, mother identity). Significant omnibus multivariate tests (i.e., Wilks' Lambda) would indicate significant differences between the representational mediator conditions and intergroup communication outcomes. Univariate main effects will be examined to determine which dependent variables are significantly different across the representational mediator 
conditions and, thus, which hypotheses (i.e., H8-H12) are supported. Significant main effects at the univariate level would suggest the categorization process influences participant mothers' levels of the dependent variables. Because previous research has consistently demonstrated a relationship between positive intergroup outcomes and the one large group recategorization, two subgroups in one large group recategorization, and separate individuals decategorization, compared to the two separate groups categorization, mediators (Gaertner et al., 1989; Gaertner et al., 1994), a priori contrasts were included in the analyses and consulted after the univariate results.

\section{Summary}

This chapter discussed the participants, procedures, instrumentation, and data analyses used across the two experimentals in this dissertation. In Study One, participant mothers were randomly assigned to view one of 13 stereotype conditions, which were designed in accordance with the most prevalent SAHM and WM stereotype profiles (Odenweller \& Rittenour, 2015, April), and completed an online questionnaire before presumably interacting with a target mother. The online questionnaire elicited participant mothers' cognitive, affective, and behavioral reactions to the stereotypes conditions in order to ascertain the varying effects of stereotypes on SAHMs' and WMs' intergroup communication outcomes. Specifically, the online questionnaire employed a series of quantitative instruments to assess previous intergroup contact ingroup identity, outgroup attitudes, affective responses (i.e., contempt, envy, and pity), behavioral responses (i.e., facilitative and harmful), intergroup anxiety, and willingness to communicate. The data obtained via the online questionnaire were analyzed via two betweensubjects, factorial MANCOVAs. The results of these analyses are reported in Chapter III of this dissertation. 
In Study Two, participant mothers were randomly assigned to view one of four representational mediator conditions, which were designed in accordance with the CIIM (Gaertner et al., 1993; Gaertner et al., 1994), and completed an online questionnaire before presumably interacting with a target mother. The online questionnaire elicited participant mothers' cognitive, affective, and behavioral reactions to the representational mediator conditions in order to ascertain the effects of transformed group boundaries on SAHMs' and WMs' intergroup communication outcomes. Specifically, the online questionnaire employed a series of quantitative instruments to assess previous intergroup contact ingroup identity, outgroup attitudes, outgroup typicality, helping, and willingness to communicate. The data obtained via the online questionnaire were analyzed via one between-subjects, factorial MANCOVA. The results of these analyses are reported in Chapter III of this dissertation. 


\section{Chapter III}

\section{Results}

\section{Study One}

\section{Pilot Study}

The frequency distributions of pilot participants' responses to the forced-choice question appear in Table 3. Overall, pilot participants were unable to successfully identify the precise stereotype conditions they were randomly selected to review. These results are not surprising given trends related to the outgroup homogeneity effect. The outgroup homogeneity effect is defined as the tendency to perceive outgroups, when compared to one's ingroups, as less variable in terms of stereotypical characteristics (Jones, Wood, \& Quattrone, 1981). Because pilot participants were undergraduate students and, thus, not mothers (and, in some cases, not women), they do not have a complex or variable understanding of motherhood and, thus, are likely to perceive mothers_-including stay-at-home, working, and all the specific variations within these two subgroups — as one homogenous social group. Additionally, pilot participants were presented the stereotype conditions outside of context, whereas participant mothers in the main study would be presented a narrative about interacting with the mothers described in the conditions. Retrospective consideration of these circumstances instilled confidence in the researcher (and her advisor) to proceed with the studies using actual mothers of young children despite the pilot results. In doing so, no changes were made to the stereotype conditions before commencing the main study as mothers recruited for the main study were expected to have more complex schemas of their ingroups and, thus, be capable of differentiating the target mother descriptions. 


\section{Table 3.}

Frequency Distributions of Forced-Choice Responses in Study One's Pilot Study

\begin{tabular}{|c|c|c|c|}
\hline \multirow[b]{2}{*}{ Stereotype Conditions } & \multirow[b]{2}{*}{$N$} & \multicolumn{2}{|c|}{$\begin{array}{l}\text { Correctly identified } \\
\text { forced-choice responses }\end{array}$} \\
\hline & & $n$ & $\%$ \\
\hline \multicolumn{4}{|l|}{ Stay-at-home Mothers } \\
\hline Busy \& Overworked & 22 & 12 & 54.55 \\
\hline Executive of the Home & 22 & 8 & 36.36 \\
\hline Lazy, Selfish, \& Lost & 10 & 9 & 90.00 \\
\hline Ideal Mother & 14 & 8 & 57.14 \\
\hline Balancing Work, Family, \& Life & 21 & 13 & 61.90 \\
\hline Non-traditional & 11 & 3 & 27.27 \\
\hline Traditional & 14 & 9 & 64.29 \\
\hline \multicolumn{4}{|l|}{ Working Mothers } \\
\hline Overextended with no free time & 22 & 9 & 40.91 \\
\hline Hardworking \& Determined & 21 & 14 & 66.67 \\
\hline Supermom & 20 & 8 & 40.00 \\
\hline Family-oriented \& Flexible & 17 & 10 & 58.82 \\
\hline Non-traditional & 24 & 5 & 20.83 \\
\hline Traditional & 24 & 13 & 54.17 \\
\hline
\end{tabular}




\section{Main Study}

Manipulation Check. Two chi-square analyses were conducted to test the qualitative distinctiveness of the stereotype conditions. The first chi-square involved the six nominal level SAHM stereotype conditions and one nominal level, multiple choice question (i.e., "based on the description you read about the other mother, which of the following options best describes her?"). Results provided evidence for a significant relationship between the SAHM stereotype conditions participant mothers were assigned and the option participant mothers chose to describe the target SAHM, $\chi^{2}(36, N=286)=1236.84, p<.001$; thus, demonstrating successful experimental manipulation for SAHM. The second chi-square involved the five nominal level WM stereotype conditions and the one nominal level, multiple choice question. Results provided evidence for a significant relationship between the WM stereotype conditions participant mothers were assigned and the option participant mothers chose to describe the target WM, $\chi^{2}(25, N=243)=919.30, p<.001$. The frequency distributions of the multiple choices responses in relation to the SAHM and WM stereotype conditions (see Tables 4 and 5) further evidenced that participant mothers correctly identified their experimental conditions in Study One.

Hypothesis testing. Two MANCOVAs were conducted to test the first series of hypotheses in Study One. The revised hypotheses predicted that - when controlling for quantity and quality of previous intergroup contact—participant SAHMs' and WMs' outgroup attitudes (H1); affective responses (i.e., contempt, admiration, pity, and envy; H4), behavioral responses (i.e., facilitative and harmful behaviors; H5), intergroup anxiety (H6), and willingness to communicate with outgroup members (H7) would differ across stereotype conditions.

First, to examine differences for the SAHM stereotype conditions, a 7 (SAHM 
Table 4.

Frequency Distributions of Stay-at-home Mother Stereotype Condition Manipulation Checks

\begin{tabular}{|c|c|c|c|c|c|c|c|c|c|c|c|c|c|c|c|}
\hline \multirow[b]{3}{*}{ Stereotype Conditions } & \multirow[b]{3}{*}{$N$} & \multicolumn{14}{|c|}{ Multiple-choice Manipulation Checks } \\
\hline & & \multicolumn{2}{|c|}{$\begin{array}{c}\text { Busy \& } \\
\text { Overworked }\end{array}$} & \multicolumn{2}{|c|}{$\begin{array}{l}\text { Executive of } \\
\text { the Home }\end{array}$} & \multicolumn{2}{|c|}{$\begin{array}{l}\text { Lazy, Selfish, } \\
\text { \& Lost }\end{array}$} & \multicolumn{2}{|c|}{ Ideal Mother } & \multicolumn{2}{|c|}{$\begin{array}{c}\text { Balancing } \\
\text { Work, Family, } \\
\text { \& Life }\end{array}$} & \multicolumn{2}{|c|}{$\begin{array}{c}\text { Non- } \\
\text { Traditional }\end{array}$} & \multicolumn{2}{|c|}{ Traditional } \\
\hline & & $n$ & $\%$ & $n$ & $\%$ & $n$ & $\%$ & $n$ & $\%$ & $n$ & $\%$ & $n$ & $\%$ & $n$ & $\%$ \\
\hline Busy \& Overworked & 35 & 34 & 97.10 & 0 & 0.00 & 0 & 0.00 & 0 & 0.00 & 1 & 2.90 & 0 & 0.00 & 0 & 0.00 \\
\hline Executive of the Home & 45 & 0 & 0.00 & 39 & 86.70 & 0 & 0.00 & 2 & 4.40 & 4 & 8.90 & 0 & 0.00 & 0 & 0.00 \\
\hline Lazy, Selfish, \& Lost & 44 & 1 & 2.30 & 0 & 0.00 & 43 & 97.70 & 0 & 0.00 & 0 & 0.00 & 0 & 0.00 & 0 & 0.00 \\
\hline Ideal Mother & 38 & 0 & 0.00 & 5 & 13.20 & 0 & 0.00 & 27 & 71.10 & 4 & 10.50 & 0 & 0.00 & 2 & 5.30 \\
\hline $\begin{array}{l}\text { Balancing Work, } \\
\text { Family, \& Life }\end{array}$ & 44 & 3 & 6.80 & 2 & 4.50 & 0 & 0.00 & 1 & 2.30 & 38 & 86.40 & 0 & 0.00 & 0 & 0.00 \\
\hline Non-traditional & 39 & 0 & 0.00 & 3 & 7.70 & 0 & 0.00 & 1 & 2.60 & 6 & 15.40 & 27 & 69.20 & 2 & 5.10 \\
\hline Traditional & 41 & 0 & 0.00 & 1 & 2.40 & 0 & 0.00 & 2 & 4.90 & 0 & 0.00 & 0 & 0.00 & 38 & 92.70 \\
\hline
\end{tabular}


Table 5.

Frequency Distributions of Working Mother Stereotype Condition Manipulation Checks

\begin{tabular}{|c|c|c|c|c|c|c|c|c|c|c|c|c|c|}
\hline \multirow[b]{3}{*}{ Stereotype Conditions } & \multirow[b]{3}{*}{$N$} & \multicolumn{12}{|c|}{ Multiple-choice Manipulation Checks } \\
\hline & & \multicolumn{2}{|c|}{$\begin{array}{c}\text { Overextended } \\
\text { with no free } \\
\text { tine }\end{array}$} & \multicolumn{2}{|c|}{$\begin{array}{l}\text { Hardworking } \\
\text { \& Determined }\end{array}$} & \multicolumn{2}{|c|}{ Supermom } & \multicolumn{2}{|c|}{$\begin{array}{c}\text { Family-oriented } \\
\& \text { Flexible }\end{array}$} & \multicolumn{2}{|c|}{ Non-traditional } & \multicolumn{2}{|c|}{ Traditional } \\
\hline & & $n$ & $\%$ & $n$ & $\%$ & $n$ & $\%$ & $n$ & $\%$ & $n$ & $\%$ & $n$ & $\%$ \\
\hline $\begin{array}{l}\text { Overextended with } \\
\text { no free time }\end{array}$ & 43 & 40 & 93.00 & 2 & 4.70 & 1 & 2.30 & 0 & 0.00 & 0 & 0.00 & 0 & 0.00 \\
\hline $\begin{array}{l}\text { Hardworking \& } \\
\text { Determined }\end{array}$ & 38 & 1 & 2.60 & 37 & 97.40 & 0 & 0.00 & 0 & 0.00 & 0 & 0.00 & 0 & 0.00 \\
\hline Supermom & 42 & 0 & 0.00 & 3 & 7.10 & 38 & 90.50 & 1 & 2.40 & 0 & 0.00 & 0 & 0.00 \\
\hline $\begin{array}{l}\text { Family-oriented \& } \\
\text { Flexible }\end{array}$ & 43 & 0 & 0.00 & 1 & 2.30 & 1 & 2.30 & 41 & 95.30 & 0 & 0.00 & 0 & 0.00 \\
\hline Non-traditional & 37 & 0 & 0.00 & 12 & 32.40 & 2 & 5.40 & 1 & 2.70 & 22 & 59.50 & 0 & 0.00 \\
\hline Traditional & 40 & 1 & 2.50 & 0 & 0.00 & 0 & 0.00 & 2 & 5.00 & 1 & 2.50 & 36 & 90.0 \\
\hline
\end{tabular}


stereotype: busy and overworked; executive of the home; lazy, selfish, and lost; ideal mother; balancing work, family, and life; non-traditional; and traditional) x 2 (participant mother group membership: SAHM and WM) between-subjects factorial MANCOVA was conducted for the eight dependent variables (i.e., outgroup attitudes; contempt, pity, and envy affective responses, facilitative and harmful behavioral responses, intergroup anxiety, and willingness to communicate), using four covariates (i.e., quantity of previous intergroup contact, quality of previous intergroup contact, ingroup identity, and mother identity). Before interpreting the MANCOVA results, univariate and multivariate homogeneity of (co)variance assumptions were evaluated using the conservative alpha level of $p<.01$ (recommended for MANCOVA's with eight dependent variables).

The Box's M test of homogeneity of covariance was significant $(F[432]=1.48, p<$ .001). This suggests the within-group variance among covariance matrices (i.e., intercorrelations among dependent variables) are unequal, which can generate misleading assessments of error variance in the subsequent analysis (Tabachnick \& Fidell, 2013). It should be noted that this test is very sensitive to cell size and number of dependent variables such that the more unequal the cell sizes and the more numerous the dependent variables (such as the current dissertation), the more unequal the covariance matrices are likely to be (Tabachnick \& Fidell, 2013). However, if the cell sizes are approximately equal (as in this dissertation), the robustness of the MANCOVA can be guaranteed and the violation of this assumption is minimal (Tabachnick \& Fidell, 2013). Given a significant Box's M, Pillai's Trace criterion (instead of Wilk’s Lambda as previously mentioned) was used to evaluate multivariate results for this MANCOVA (Tabachnick \& Fidell, 2013).

The Levene's test of homogeneity of variance was significant for five of the eight 
dependent variables: outgroup attitudes $(p=.04)$, contempt $(p<.001)$, envy $(p<.001)$, pity $(p=$ $.80)$, facilitative behaviors $(p<.001)$, harmful behaviors $(p<.01)$, intergroup anxiety $(p<.05)$, and willingness to communicate $(p=.24)$. These results demonstrate that the level of variance across several dependent variables for each stereotype and group condition is unequal. The following results should be interpreted with violated assumption in mind.

The Pillai's Trace results at the multivariate level were examined for the stereotype conditions, group conditions, and stereotype $\mathrm{x}$ group interaction. The multivariate results revealed a significant main effect for the stereotype conditions $\left(F[48,1596]=8.02, p<.001, \eta_{p}^{2}\right.$ $=.19$, power $=1.00)$. The multivariate results revealed non-significant main effects for the group conditions $\left(F[8,261]=.95, p=.48, \eta_{p}^{2}=.03\right.$, power $\left.=.44\right)$ and the stereotype $\mathrm{x}$ group interaction $\left(F[48,1596]=1.11, p=.28, \eta_{p}^{2}=.03\right.$, power $\left.=.99\right)$. The multivariate results revealed quality of previous intergroup contact as a significant covariate $(F[8,261]=3.81, p<$ $.001, \eta_{p}^{2}=.11$, power $\left.=.99\right)$ and ingroup identity $\left(F[8,261]=2.31, p=.02, \eta_{p}^{2}=.07\right.$, power $=$ $.88)$, mother identity $\left(F[8,261]=.64, p=.74, \eta_{p}^{2}=.02\right.$, power $\left.=.30\right)$, and quantity of previous intergroup contact $\left(F[8,261]=1.76, p=.09, \eta_{p}^{2}=.05\right.$, power $\left.=.75\right)$ as a non-significant covariates.

Because the stereotype condition was the only independent variable that obtained significant multivariate results, it was the only independent variable examined at the univariate level for each dependent variable. The results revealed significant main effects for seven of the eight dependent variables: outgroup attitudes $\left(F[6,268]=16.81, p<.001, \eta_{p}^{2}=.27\right.$, power $=$ $1.00)$, contempt $\left(F[6,268]=22.18, p<.001, \eta_{p}^{2}=.33\right.$, power $\left.=1.00\right)$, envy $(F[6,268]=8.36, p$ $<.001, \eta_{p}^{2}=.16$, power $\left.=1.00\right)$, pity $\left(F[6,268]=8.49, p<.001, \eta_{p}^{2}=.16\right.$, power $\left.=1.00\right)$, facilitative behaviors $\left(F[6,268]=19.57, p<.001, \eta_{p}^{2}=.31\right.$, power $\left.=1.00\right)$, harmful behaviors 
$\left(F[6,268]=26.49, p<.001, \eta_{p}^{2}=.37\right.$, power $\left.=1.00\right)$, intergroup anxiety $(F[6,268]=14.29, p<$ $.001, \eta_{p}^{2}=.24$, power $\left.=1.00\right)$, and willingness to communicate $\left(F[6,268]=1.77, p=.11, \eta_{p}^{2}=\right.$ .04 , power $=.67$ ). Because this study was primarily exploratory, post hoc analyses (i.e., Bonferroni corrections) were conducted to further explore the differences among the seven dependent variables that obtained significant univariate main effects across the seven SAHM stereotype conditions. Table 6 includes all of the means and standard deviations for the multiple pairwise comparisons discussed in this section. SAHM stereotype conditions not mentioned in this section were not significantly different from one another.

The results of multiple pairwise comparisons demonstrated two major trends for outgroup attitudes. First, participant mothers who viewed the lazy, selfish, and lost stereotype condition reported significantly less favorable outgroup attitudes compared to participant mothers who viewed all other stereotype conditions. Second, participant mothers who viewed the ideal mother stereotype condition reported significantly less favorable outgroup attitudes compared to participant mothers who viewed the busy and overworked stereotype condition.

The results of multiple pairwise comparisons demonstrated two major trends for contempt. First, participant mothers who viewed the lazy, selfish, and lost stereotype condition reported significantly higher levels of contempt compared to participant mothers who viewed all other stereotype conditions. Second, participant mothers who viewed the ideal mother stereotype condition reported significantly higher levels of contempt compared to participant mothers who viewed the non-traditional stereotype condition.

The results of multiple pairwise comparisons demonstrated two major trends for envy. First, participant mothers who viewed the lazy, selfish, and lost stereotype condition reported significantly lower levels of envy compared to participant mothers who viewed the busy and 


\section{Table 6.}

Bonferroni's Adjusted Multiple Pairwise Comparisons for Stay-at-home Mother Stereotype Conditions across Dependent Variables

\begin{tabular}{|c|c|c|c|c|c|c|c|}
\hline Variables & $\begin{array}{c}\text { Busy \& } \\
\text { Overworked } \\
(n=35)\end{array}$ & $\begin{array}{l}\text { Executive of } \\
\text { the Home } \\
(n=45)\end{array}$ & $\begin{array}{l}\text { Lazy, Selfish, } \\
\quad \& \text { Lost } \\
(n=44)\end{array}$ & $\begin{array}{l}\text { Ideal Mother } \\
\quad(\mathrm{n}=38)\end{array}$ & $\begin{array}{l}\text { Balancing Work, } \\
\text { Family, \& Life } \\
(n=44)\end{array}$ & $\begin{array}{l}\text { Non-traditional } \\
\quad(n=39)\end{array}$ & $\begin{array}{l}\text { Traditional } \\
(n=41)\end{array}$ \\
\hline Outgroup Attitudes $^{\dagger}$ & $7.94(2.30)^{\mathrm{bc}}$ & $7.22(2.38)^{\mathrm{b}}$ & $2.70(1.69)^{\mathrm{a}}$ & $5.66(2.80)^{\mathrm{bd}}$ & $6.98(2.31)^{\mathrm{b}}$ & $7.18(2.74)^{\mathrm{b}}$ & $6.63(3.25)^{\mathrm{b}}$ \\
\hline Contempt & $1.97(0.43)^{\mathrm{b}}$ & $1.85(0.57)^{\mathrm{b}}$ & $3.55(0.61)^{\mathrm{a}}$ & $2.30(0.86)^{\mathrm{bc}}$ & $1.74(0.61)^{\mathrm{b}}$ & $1.76(0.70)^{\mathrm{bd}}$ & $2.09(0.75)^{\mathrm{b}}$ \\
\hline Pity & $2.42(1.12)^{\mathrm{c}}$ & $1.92(1.11)^{\mathrm{b}}$ & $3.89(1.56)^{\mathrm{a}}$ & $2.32(1.22)^{\mathrm{b}}$ & $2.46(1.30)$ & $1.67(1.24)^{\mathrm{bd}}$ & $2.51(1.53)^{\mathrm{b}}$ \\
\hline Envy & $2.11(1.10)^{\mathrm{b}}$ & $2.38(1.59)^{\mathrm{b}}$ & $1.18(0.45)^{\mathrm{a}}$ & $3.30(1.73)^{\mathrm{bc}}$ & $2.00(1.44)^{\mathrm{d}}$ & $1.96(1.29)^{\mathrm{d}}$ & $1.73(1.11)^{\mathrm{d}}$ \\
\hline Facilitative Behaviors & $3.07(0.58)^{\mathrm{c}}$ & $4.97(1.19)^{\mathrm{bd}}$ & $3.43(1.33)^{\mathrm{a}}$ & $4.74(1.19)^{\mathrm{bd}}$ & $5.13(1.09)^{\mathrm{bd}}$ & $5.06(1.34)^{\mathrm{bd}}$ & $4.98(1.37)^{\mathrm{bc}}$ \\
\hline Harmful Behaviors & $2.53(0.60)^{\mathrm{bc}}$ & $1.27(0.48)^{\mathrm{bd}}$ & $1.80(0.78)^{\mathrm{ad}}$ & $1.53(0.64)^{\mathrm{d}}$ & $1.22(0.37)^{\mathrm{bd}}$ & $1.30(0.50)^{\mathrm{d}}$ & $1.360 .65^{\mathrm{d}}$ \\
\hline Intergroup Anxiety & $3.73\left(0.45^{\mathrm{c}}\right.$ & $2.99(1.10)^{\mathrm{bdf}}$ & $4.41(0.81)^{\mathrm{a}}$ & $3.80(1.32)^{\mathrm{e}}$ & $2.90(1.07)^{\mathrm{bdf}}$ & $2.94(1.10)^{\mathrm{bdf}}$ & $3.201 .24^{\mathrm{b}}$ \\
\hline $\begin{array}{l}\text { Willingness to } \\
\text { Communicate }\end{array}$ & 5.491 .17 & $5.62(0.92)$ & $5.18(1.14)$ & $5.09(1.05)$ & $5.40(1.02)$ & $5.27(0.98)$ & 5.370 .97 \\
\hline
\end{tabular}

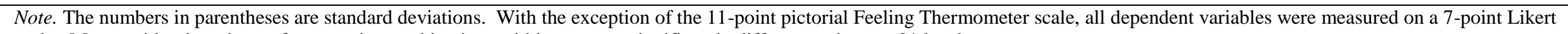
scale. Means with a-b, c-d, or e-f superscript combinations within rows are significantly different at the $p<.01$ level.

${ }^{\dagger}$ Higher means for outgroup attitudes represent positive outgroup attitudes. 
overworked and executive of the home stereotype conditions. Second, the participant mothers who viewed the ideal mother stereotype condition reported significantly higher levels of envy compared to participant mothers who viewed the lazy, selfish, and lost; balancing work, family, and life; non-traditional; and traditional stereotype conditions.

The results of multiple pairwise comparisons demonstrated two major trends for pity. First, participant mothers who viewed the lazy, selfish, and lost stereotype condition reported significantly higher levels of pity compared to participant mothers who viewed the executive of the home, ideal mother, non-traditional, and traditional stereotype conditions. Second, participant mothers who viewed the busy and overworked stereotype condition reported significantly higher levels of pity compared to participant mothers who viewed the nontraditional stereotype condition.

The results of multiple pairwise comparisons demonstrated two major trends for facilitative behaviors. First, participant mothers who viewed the lazy, selfish, and lost stereotype condition reported significantly lower levels of facilitative behaviors compared to participant mothers who viewed the executive of the home; ideal mother; balancing work, family, and life; non-traditional, and traditional stereotype conditions. Second, participant mothers who viewed the busy and overworked stereotype condition reported significantly lower levels of facilitative behaviors compared to participant mothers who viewed the executive of the home; ideal mother; balancing work, family, and life; non-traditional, and traditional stereotype conditions.

The results of multiple pairwise comparisons demonstrated two major trends for harmful behaviors. First, participant mothers who viewed the lazy, selfish, and lost stereotype condition reported significantly higher levels of harmful behaviors compared to participant mothers who viewed the executive of the home and balancing work, family, and life stereotype conditions. 
Second, participant mothers who viewed the busy and overworked stereotype condition reported significantly higher levels of harmful behaviors compared to participant mothers who viewed all other stereotype conditions.

The results of multiple pairwise comparisons demonstrated three major trends for intergroup anxiety. First, participant mothers who viewed the lazy, selfish, and lost stereotype condition reported significantly higher levels of intergroup anxiety compared to participant mothers who viewed the executive of the home; balancing work, family, and life; non-traditional; and traditional stereotype conditions. Second, participant mothers who viewed the busy and overworked stereotype condition reported significantly higher levels of intergroup anxiety compared to participant mothers who viewed the executive of the home; balancing work, family, and life; and non-traditional stereotype conditions. Third, participant mothers who viewed the ideal mother stereotype condition reported significantly higher levels of intergroup anxiety compared to participant mothers who viewed the executive of the home; balancing work, family, and life; and non-traditional stereotype conditions.

Second, to examine differences for the WM stereotype conditions, a 6 (WM stereotypes: overextended with no free time, hardworking and determined, supermom, flexible and familyoriented, non-traditional, and traditional) x 2 (participant mother ingroup/outgroup membership: SAHM and WM) between-subjects factorial MANCOVA was conducted for the eight dependent variables (i.e., outgroup attitudes; contempt, pity, and envy affective responses, facilitative and harmful behavioral responses, intergroup anxiety, and willingness to communicate), using four covariates (i.e., quantity of previous intergroup contact, quality of previous intergroup contact, ingroup identity, and mother identity). Before interpreting the MANCOVA results, univariate and multivariate homogeneity of (co)variance assumptions were evaluated using the conservative 
alpha level of $p<.01$ (recommended for MANCOVA's with eight dependent variables).

The Box's M test of homogeneity of covariance was significant $(F[396]=1.22, p<.01)$. This suggests the within-group variance among covariance matrices (i.e., intercorrelations among dependent variables) are unequal. As previously mentioned, unequal covariance can generate misleading assessments of error variance, especially in analyses with unequal cell sizes and a substantial number of dependent variables (Tabachnick \& Fidell, 2013). However, the nearly equal cell sizes in this dissertation guarantees the robustness of the subsequent MANCOVA (Tabachnick \& Fidell, 2013). Given a significant Box's M, Pillai's Trace criterion was used to evaluate multivariate results for this MANCOVA (Tabachnick \& Fidell, 2013).

The Levene's test of homogeneity of variance was significant for five of the eight dependent variables: outgroup attitudes $(p=.09)$, contempt $(p=.01)$, envy $(p<.01)$, pity $(p<$ $.001)$, facilitative behaviors $(p<.01)$, harmful behaviors $(p<.001)$, intergroup anxiety $(p=.48)$, and willingness to communicate $(p=.64)$. These results demonstrate that the level of variance across several dependent variables for each stereotype and group condition is unequal. The following results should be interpreted with violated assumption in mind.

The Pillai's Trace results at the multivariate level were examined for the stereotype conditions, group conditions, and stereotype $\mathrm{x}$ group interaction. The multivariate results revealed a significant main effect for the stereotype conditions $\left(F[40,1120]=4.72, p<.001, \eta_{p}^{2}\right.$ $=.14$, power $=1.00)$ and a significant stereotype $\mathrm{x}$ group interaction $(F[40,1120]=1.72, p<$ $.01, \eta_{p}^{2}=.06$, power $\left.=1.00\right)$. The multivariate results revealed a non-significant main effect for the group conditions $\left(F[8,220]=1.43, p=.19, \eta_{p}^{2}=.05\right.$, power $\left.=.64\right)$. The multivariate results revealed ingroup identity $\left(F[8,220]=1.52, p=.15, \eta_{p}^{2}=.05\right.$, power $\left.=.67\right)$, mother identity $(F[8$, $220]=1.53, p=.15, \eta_{p}^{2}=.05$, power $\left.=.68\right)$, quantity of previous intergroup $\operatorname{contact}(F[8,220]=$ 
$1.36, p=.22, \eta_{p}^{2}=.05$, power $\left.=.61\right)$, and quality of previous intergroup contact $(F[8,220]=$ $1.94, p=.06, \eta_{p}^{2}=.07$, power $\left.=.80\right)$ as non-significant covariates.

Because the stereotype conditions and stereotype $\mathrm{x}$ group interaction both obtained significant multivariate results, they were examined at the univariate level for each dependent variable. The univariate results revealed significant main effects for seven of the eight dependent variables: outgroup attitudes $\left(F[5,227]=10.79, p<.001\right.$, partial $\eta^{2}=.19$, power $\left.=1.00\right)$, contempt $(F[5$, $227]=4.81, p<.001, \eta_{p}^{2}=.10$, power $\left.=.98\right)$, envy, $\left(F[5,227]=4.16, p<.01, \eta_{p}^{2}=.08\right.$, power $=$ $.96)$, pity $\left(F[5,227]=21.56, p<.001, \eta_{p}^{2}=.32\right.$, power $\left.=1.00\right)$, facilitative behaviors $(F[5,227]$ $=4.74, p<.001, \eta_{p}^{2}=.10$, power $\left.=.98\right)$, and intergroup anxiety $\left(F[5,227]=3.80, p<.01, \eta_{p}^{2}=\right.$ .08 , power $=.94)$. Harmful behaviors $\left(F[5,227]=2.90, p=.02, \eta_{p}^{2}=.06\right.$, power $\left.=.84\right)$ and willingness to communicate $\left(F[5,227]=2.47, p=.03, \eta_{p}^{2}=.05\right.$, power $\left.=.77\right)$ were not significant at the univariate level. Because this dissertation was primarily exploratory, post hoc analyses (i.e., Bonferroni corrections) were conducted to further explore the differences among the seven dependent variables that obtained significant univariate main effects across the six WM stereotype conditions. Table 7 includes all of the means and standard deviations for the multiple pairwise comparisons discussed in this section. WM stereotype conditions not mentioned in this section were not significantly different from one another.

The results of multiple pairwise comparisons demonstrated two major trends for outgroup attitudes. First, participant mothers who viewed the traditional stereotype condition reported significantly less favorable outgroup attitudes compared to participant mothers who viewed the overextended with no free time, hardworking and determined, family-oriented and flexible, and non-traditional stereotype conditions. Second, participant mothers who viewed the supermom stereotype condition reported significantly less favorable outgroup attitudes compared to 


\section{Table 7.}

Bonferroni's Adjusted Multiple Pairwise Comparisons for Working Mothers Stereotype Conditions across Dependent Variables

\begin{tabular}{|c|c|c|c|c|c|c|}
\hline Variables & $\begin{array}{l}\text { Overextended } \\
\text { with no free time } \\
\quad(n=43)\end{array}$ & $\begin{array}{c}\text { Hardworking \& } \\
\text { Determined } \\
(n=38)\end{array}$ & $\begin{array}{l}\text { Supermom } \\
\quad(n=42)\end{array}$ & $\begin{array}{l}\text { Family-oriented } \\
\text { \& Flexible } \\
(n=43)\end{array}$ & $\begin{array}{l}\text { Non-traditional } \\
\quad(n=37)\end{array}$ & $\begin{array}{l}\text { Traditional } \\
\quad(n=40)\end{array}$ \\
\hline Outgroup Attitudes ${ }^{\dagger}$ & $7.91(2.51)^{\mathrm{bd}}$ & $7.39(2.22)^{b}$ & $5.52(2.22)^{\mathrm{c}}$ & $8.67(1.91)^{b d}$ & $8.14(2.41)^{b d}$ & $5.53(2.34)^{\mathrm{a}}$ \\
\hline Contempt & $1.91(0.61)$ & $1.71(0.56)$ & $2.20(0.58)$ & $1.70(0.54)$ & $1.62(0.68)^{\mathrm{a}}$ & $2.19(0.69)^{b}$ \\
\hline Pity & $4.79(1.37)^{\mathrm{a}}$ & $2.43(1.33)^{b}$ & $2.07(1.21)^{\mathrm{b}}$ & $1.92(1.22)^{\mathrm{b}}$ & $1.89(1.11)^{\mathrm{b}}$ & $2.65(1.40)^{b}$ \\
\hline Envy & $1.31(0.50)^{\mathrm{a}}$ & $2.11(1.32)$ & $2.82(1.64)^{\mathrm{b}}$ & $1.87(1.52)$ & $1.97(1.33)$ & $1.99(1.27)$ \\
\hline Facilitative Behaviors & $5.40(1.02)^{\mathrm{b}}$ & $5.01(1.32)$ & $4.32(1.47)$ & $5.31(1.34)^{b}$ & $5.22(0.85)$ & $4.30(1.20)^{\mathrm{a}}$ \\
\hline Harmful Behaviors & $1.18(0.33)$ & $1.14(0.32)$ & $1.48(0.64)$ & $1.20(0.49)$ & $1.28(0.62)$ & $1.51(0.67)$ \\
\hline Intergroup Anxiety & $3.15(0.89)^{\mathrm{b}}$ & $2.90(0.91)$ & $3.52(1.09)$ & $2.48(0.87)^{\mathrm{a}}$ & $2.91(0.97)$ & $3.28(0.88)^{\mathrm{b}}$ \\
\hline $\begin{array}{l}\text { Willingness to } \\
\text { Communicate }\end{array}$ & $5.33(1.04)$ & $5.82(0.85)$ & $5.45(0.89)$ & $5.68(0.92)$ & $5.47(0.96)$ & $5.31(1.02)$ \\
\hline
\end{tabular}


participant mothers who viewed the overextended with no free time, family-oriented and flexible, and non-traditional stereotype condition.

The results of pairwise comparisons demonstrated one major trend for contempt. Participant mothers who viewed the traditional stereotype condition reported significantly higher levels of contempt compared to participant mothers who viewed the non-traditional stereotype condition.

The results of pairwise comparisons demonstrated one major trend for envy. Participant mothers who viewed the supermom stereotype condition reported significantly higher levels of envy compared to participant mothers who viewed the overextended with no free time stereotype condition.

The results of pairwise comparisons demonstrated one major trend for pity. Participant mothers who viewed the overextended with no free time stereotype condition reported significantly higher levels of pity compared to participant mothers who viewed all other stereotype conditions.

The results of pairwise comparisons demonstrated one major trend for facilitative behaviors. Participant mothers who viewed the traditional stereotype condition reported significantly lower levels of facilitative behaviors compared to participant mothers who viewed the overextended with no free time and family-oriented and flexible stereotype conditions.

The results of pairwise comparisons demonstrated one major trend for intergroup anxiety. Participant mothers who viewed the family-oriented and flexible stereotype condition reported significantly lower levels of intergroup anxiety compared to participant mothers who viewed the overextended with no free time and traditional stereotype conditions.

The main effects for two dependent variables, facilitative behaviors and intergroup 
anxiety, are qualified by a significant stereotype $\mathrm{x}$ group interaction revealed at the univariate level: facilitative behaviors $\left(F[5,229]=4.87, p<.001, \eta_{p}^{2}=.10\right.$, power $\left.=.98\right)$ and intergroup anxiety $\left(F[5,229]=3.16, p<.01, \eta_{p}^{2}=.07\right.$, power $\left.=.88\right)$. Separate MANCOVAs (i.e., simple effects models) were conducted to further examine the effects of facilitative behaviors and intergroup anxiety for one independent variable (i.e., participant mothers' group membership) at each level of the other independent variable (i.e., WM stereotype descriptions). Table 8 includes the means and standard deviations for the multiple pairwise comparisons discussed in this section.

The results of the simple effects model for facilitative behaviors revealed higher levels of facilitative behaviors for WMs for the overextended with no free time, hardworking and determined, and supermom stereotype conditions and higher levels of facilitative behaviors for SAHMs for the traditional, non-traditional, and family-oriented and flexible stereotype conditions. The results of the simple effects model for intergroup anxiety revealed higher levels of intergroup anxiety for WMs for the supermom and family-oriented and flexible stereotype conditions and higher levels of intergroup anxiety for SAHMs for all other stereotype conditions. Although MANCOVA results did not provide any evidence to suggest SAHMs' and WMs' willingness to communicate differ based on stereotype conditions with the continuous variable, it is worth examining the results of the behavioral assessment of willingness to communicate (i.e., participant mothers' advancement to the online interaction at the end of the questionnaire). Chisquare results of participant mothers' willingness to communicate via this behavioral operationalization are reported in Table 9.

Two additional hypotheses examined ingroup favoritism. $\mathrm{H} 2$ predicted that participant SAHMs and WMs would report more positive attitudes when the target mother was a member of 
Table 8.

Descriptive Statistics for the Simple Effects Models of Facilitative Behaviors and Intergroup Anxiety across Working Mother Stereotype Conditions for Participant Working Mothers and Stay-at-home Mothers

\begin{tabular}{|c|c|c|c|c|}
\hline \multirow[b]{2}{*}{ Stereotype Conditions } & \multicolumn{2}{|c|}{ Facilitative Behaviors } & \multicolumn{2}{|c|}{ Intergroup Anxiety } \\
\hline & Working Mothers & Stay-at-home Mothers & Working Mothers & Stay-at-home Mothers \\
\hline Overextended with no free time & $5.43(1.17)$ & $5.37(0.81)$ & $2.78(0.73)$ & $3.62(0.86)$ \\
\hline Hardworking \& Determined & $5.53(0.99)$ & $4.44(1.43)$ & $2.83(0.99)$ & $2.98(0.84)$ \\
\hline Supermom & $4.47(1.59)$ & $4.03(1.21)$ & $3.63(1.13)$ & $3.31(1.03)$ \\
\hline Family-oriented \& Flexible & $4.20(1.14)$ & $4.41(1.29)$ & $3.21(0.85)$ & $3.36(0.93)$ \\
\hline Non-traditional & $5.15(0.83)$ & $5.31(0.90)$ & $2.56(0.91)$ & $3.28(0.92)$ \\
\hline Traditional & $4.58(1.45)$ & $5.94(0.85)$ & $2.73(0.89)$ & $2.26(0.81)$ \\
\hline
\end{tabular}

Note. The numbers in parentheses are standard deviations. The dependent variables (i.e., facilitative behaviors and intergroup anxiety) were measured on a 7-point, Likert scale. 
the participant mothers' ingroup compared to when the target mother was a member of the participant mothers' outgroup. The results of two one-tailed, independent $t$-tests revealed significant differences between participant SAHMs' $(M=6.89, S D=3.02)$ and participant WMs' $(M=5.88, S D=2.91)$ attitudes toward target SAHMs $(t[284]=-2.80, p<.05)$, but no significant differences between participant SAHMs' $(M=7.21, S D=2.52)$ and participant WMs' $(M=7.18, S D=2.63)$ attitudes toward target WMs $(t[241]=-.12, p=.91)$. Thus, H2 was only partially supported as participant SAHMs were the only group to display ingroup favoritism. H3 predicted that participant SAHMs would report more positive attitudes for target SAHMs compared to participant WMs' attitudes for target WMs. Fully consistent with H3's reasoning, $\mathrm{H} 2$ results evidence SAHMs as the only group to display ingroup favoritism. However, because $\mathrm{H} 2$ results demonstrate an absence of ingroup favoritism for WMs, there is no WM ingroup favoritism upon which to draw comparisons with SAHM ingroup favoritism to fully assess H3. Due to these results, H3 is partially supported.

\section{Study Two}

\section{Pilot Study}

The frequency distributions based on pilot study participants responses to the forcedchoice question appear in Table 10. As discussed in Study One, the outgroup homogeneity effect (Jones et al., 1981) likely played a role in the pilot study results. Because pilot participants were undergraduate students or mothers of older children (and thus, less likely to identify as a SAHM or WM), it is not surprising that pilot participants were unable to successfully identify the representational mediators. Additionally, pilot participants were presented the messages outside of context, whereas participant mothers in the main study would be presented a narrative about interacting with the mothers who had allegedly sent the messages. Retrospective consideration 
Table 9.

Frequency Distributions of Participant Mothers' Advancement to the Online Interaction

\begin{tabular}{|c|c|c|c|c|c|c|c|c|c|c|c|}
\hline \multirow[t]{3}{*}{ Stereotype Condition } & \multirow[t]{3}{*}{$N$} & \multicolumn{5}{|c|}{ Participant Working Mothers } & \multicolumn{5}{|c|}{ Participant Stay-at-home Mothers } \\
\hline & & \multirow[b]{2}{*}{$n$} & \multicolumn{2}{|c|}{$\begin{array}{l}\text { Advancement to the } \\
\text { online interaction }\end{array}$} & \multicolumn{2}{|c|}{ Exit Study } & \multicolumn{3}{|c|}{$\begin{array}{l}\text { Advancement to the } \\
\text { online interaction }\end{array}$} & \multicolumn{2}{|c|}{ Exit Study } \\
\hline & & & $N$ & $\%$ & $N$ & $\%$ & $n$ & $N$ & $\%$ & $N$ & $\%$ \\
\hline \multicolumn{12}{|l|}{ Stay-at-home Mother Stereotypes } \\
\hline Busy \& Overworked & 35 & 20 & 19 & 95.00 & 1 & 5.00 & 15 & 14 & 93.30 & 1 & 6.70 \\
\hline Executive of the Home & 45 & 27 & 23 & 85.20 & 4 & 14.80 & 18 & 18 & 100.00 & 0 & 0.00 \\
\hline Lazy, Selfish, \& Lost & 44 & 34 & 30 & 88.20 & 4 & 11.80 & 10 & 8 & 80.00 & 2 & 20.00 \\
\hline Ideal Mother & 38 & 25 & 23 & 92.00 & 2 & 8.00 & 13 & 13 & 100.00 & 0 & 0.00 \\
\hline Balancing Work, Family, \& Life & 44 & 21 & 21 & 100.00 & 0 & 0.00 & 23 & 23 & 100.00 & 0 & 0.00 \\
\hline Non-traditional & 39 & 25 & 23 & 92.00 & 2 & 8.00 & 14 & 14 & 100.00 & 0 & 0.00 \\
\hline Traditional & 41 & 21 & 19 & 90.50 & 2 & 9.50 & 20 & 20 & 100.00 & 0 & 0.00 \\
\hline \multicolumn{12}{|l|}{ Working Mother Stereotypes } \\
\hline Overextended with no free time & 43 & 24 & 22 & 91.70 & 2 & 8.30 & 19 & 19 & 100.00 & 0 & 0.00 \\
\hline Hardworking \& Determined & 38 & 20 & 20 & 100.00 & 0 & 0.00 & 18 & 17 & 94.40 & 1 & 5.60 \\
\hline Supermom & 42 & 27 & 25 & 92.60 & 2 & 7.40 & 15 & 15 & 100.00 & 0 & 0.00 \\
\hline Family-oriented \& Flexible & 43 & 20 & 19 & 95.00 & 1 & 5.00 & 23 & 22 & 95.70 & 1 & 4.30 \\
\hline Non-traditional & 37 & 19 & 19 & 100.00 & 0 & 0.00 & 18 & 17 & 94.40 & 1 & 5.60 \\
\hline Traditional & 40 & 21 & 21 & 100.00 & 0 & 0.00 & 19 & 19 & 100.00 & 0 & 0.00 \\
\hline Totals & 529 & 304 & 284 & & 20 & & 225 & 219 & & 6 & \\
\hline
\end{tabular}

Note. Working mothers: $\chi 2(12, N=304)=10.95, p=.53$; Stay-at-home mothers: $\chi 2(12, N=225)=17.77, p=.12$ 


\section{Table 10.}

Frequency Distributions of Forced-Choice Responses in Study Two's Pilot Study

\begin{tabular}{lcccc}
\hline & \multicolumn{3}{c}{$\begin{array}{c}\text { Correctly identified } \\
\text { forced-choice responses }\end{array}$} \\
\cline { 3 - 5 } Representational Mediator Conditions & $N$ & $n$ & $\%$ \\
\hline One group recategorization & 8 & 4 & 50.00 \\
Two subgroups in one group recategorization & 12 & 4 & 33.33 \\
Two group categorization & 13 & 5 & 38.46 \\
Separate individuals decategorization & 12 & 1 & 8.33 \\
\hline
\end{tabular}


of these circumstances instilled confidence in the researcher (and her advisor) to proceed with the studies using actual mothers of young children despite the pilot results. In doing so, no changes were made to the representational mediator conditions before commencing the main study as mothers recruited for the main study were expected to have more complex schemas of their ingroups and, thus, be capable of differentiating the mediators.

\section{Main Study}

Manipulation Check. One chi-square analyses was conducted to test the qualitative distinctiveness of the representational mediator conditions. The chi-square involved the four nominal level representational mediator conditions (i.e., one group recategorization, two subgroups in one group recategorization, two group categorization, and separate individuals decategorization) and one nominal level, multiple choice question (i.e., "After reading the other mother's message, I feel as if we are...”). Results evidence a significant relationship between the representational mediator conditions participant mothers were assigned and the option participant mothers chose to describe their categorization process, $\chi^{2}(9, N=156)=35.47, p<$ .001 ; thus, demonstrating successful experimental manipulation in Study Two. However, closer examination of the frequency distributions of the multiple choices responses in relation to the representational mediator conditions (see Table 11) suggest participant mothers did not correctly identify their experimental conditions in Study Two.

Hypothesis Testing. One MANCOVA was conducted to test the five hypotheses in Study Two. These hypotheses predicted that, following an intergroup contact experience, participant SAHMs' and WMs' reliance on stereotypes (H8), outgroup attitudes (H9), outgroup typicality (H10), helping (H11), and willingness to communicate with outgroup members (H12) would differ across representational mediator conditions put forth in the common ingroup 


\section{Table 11.}

Frequency Distributions of Representational Mediator Condition Manipulation Checks

\begin{tabular}{|c|c|c|c|c|c|c|c|c|c|}
\hline \multirow[b]{3}{*}{ Representational Mediator Conditions } & \multirow[b]{3}{*}{$N$} & \multicolumn{8}{|c|}{ Multiple-choice Manipulation Checks } \\
\hline & & \multicolumn{2}{|c|}{$\begin{array}{c}\text { One Group } \\
\text { Recategorization }\end{array}$} & \multicolumn{2}{|c|}{$\begin{array}{l}\text { Two Subgroups } \\
\text { in One } \\
\text { Recategorization }\end{array}$} & \multicolumn{2}{|c|}{$\begin{array}{c}\text { Two Group } \\
\text { Categorization }\end{array}$} & \multicolumn{2}{|c|}{$\begin{array}{l}\text { Unique \& Separate } \\
\text { Individuals } \\
\text { Decategorization }\end{array}$} \\
\hline & & $N$ & $\%$ & $N$ & $\%$ & $N$ & $\%$ & $N$ & $\%$ \\
\hline One Group Recategorization & 35 & 21 & 60.00 & 5 & 14.30 & 0 & 0.00 & 9 & 25.70 \\
\hline Two Subgroups in One Recategorization & 39 & 14 & 35.90 & 18 & 46.20 & 0 & 0.00 & 7 & 17.90 \\
\hline Two Group Categorization & 41 & 9 & 22.00 & 17 & 41.50 & 6 & 85.70 & 9 & 22.00 \\
\hline Unique \& Separate Individuals Decategorization & 39 & 24 & 61.50 & 5 & 12.80 & 1 & 2.60 & 9 & 23.10 \\
\hline
\end{tabular}


identity model (CIIM; Gaertner et al., 1993; Gaertner et al., 1994).

The one-way, between subjects MANCOVA examined differences between representational mediator conditions (i.e., one group recategorization, two subgroups in one group recategorization, two group categorization, and separate individuals decategorization) for the five dependent variables (i.e., stereotype reliance, outgroup attitudes, outgroup typicality, helping, and willingness to communicate), using four covariates (i.e., quantity of previous intergroup contact, quality of previous intergroup contact, ingroup identity, and mother identity). Before interpreting the MANCOVA results, uni- and multivariate homogeneity of (co)variance assumptions were evaluated using the conservative alpha level of $p<.01$ (recommended for MANCOVA's with five dependent variables).

The Box's M test of homogeneity of covariance was not significant $(F[45]=47.66, p=$ .48). This suggests the within-group variance among covariance matrices (i.e., intercorrelations among dependent variables) are equal, which is ideal for assessing between-group differences across multiple dependent variables (Tabachnick \& Fidell, 2013). Upon satisfying this multivariate assumption of covariate homogeneity, the Wilk's Lambda criterion was used to evaluate multivariate results for this MANCOVA (Tabachnick \& Fidell, 2013).

The Levene's test of homogeneity of variance was not significant for four of the five dependent variables: stereotype reliance $(p=.10)$, outgroup attitudes $(p=.67)$, outgroup typicality $(p<.01)$, helping $(p=.83)$, and willingness to communicate $(p=.30)$. These results demonstrate that the level of variance across all but one dependent variable for each stereotype and group condition is equal (Tabachnick \& Fidell, 2013). These results satisfy the univariate assumption of variance homogeneity. However, the following results should be interpreted with the variance of outgroup typicality and, thus, a violated univariate assumption, in mind. 
The Wilks' Lambda results at the multivariate level revealed a main effect for the representational mediator conditions that approached the conservative, a priori $p$ value $(F[15$, $392.40]=1.74, p=.04, \eta_{p}^{2}=.06$, power $=.89$ ). The multivariate results revealed four nonsignificant covariates: quantity of previous intergroup contact $\left(F[5,142]=1.02, p=.41, \eta_{p}^{2}=\right.$ .04 , power $=.36)$, quality of previous intergroup contact $\left(F[5,142]=2.16, p=.06, \eta_{p}^{2}=.07\right.$, power $=.70)$, ingroup identity $\left(F[5,142]=1.93, p=.09, \eta_{p}^{2}=.06\right.$, power $\left.=.64\right)$, and mother identity $\left(F[5,142]=1.14, p=.34, \eta_{p}^{2}=.04\right.$, power $\left.=.40\right)$.

The univariate results revealed significant main effects for only one of the five dependent variables: outgroup typicality $\left(F[3,146]=4.70, p<.01, \eta_{p}^{2}=.09\right.$, power $\left.=.89\right)$. The other four dependent variables were non-significant at the univariate level: stereotype reliance $(F[3,146]=$ $.71, p=.55, \eta_{p}^{2}=.01$, power $\left.=.20\right)$, outgroup attitudes $\left(F[3,146]=1.45, p=.23, \eta_{p}^{2}=.03\right.$, power $=.38)$, , helping $\left(F[3,146]=.36, p=.78, \eta_{p}^{2}=.01\right.$, power $\left.=.12\right)$, and willingness to communicate $\left(F[3,146]=1.39, p=.25, \eta_{p}^{2}=.03\right.$, power $\left.=.36\right)$.

Because this study was designed in accordance with previous research on the CIIM (e.g., Gaertner et al., 1999; Gaertner et al., 1989), planned contrasts were included in the analyses to examine the hypothesized differences between the two group categorization and the other three (i.e., one group recategorization, two subgroups in one group recategorization, and separate individuals decategorization) representational mediator conditions in relation to outgroup typicality. The results of the planned contrasts demonstrated outgroup typicality was significantly different across the representational mediator conditions, but neither as hypothesized nor universally below the conservative, a priori $p$ value. Table 12 includes the planned contrast results and Table 13 includes all of the means and standard deviations for the representational mediator conditions. 


\section{Summary}

This chapter presented the results of the two, independent experiments employed in this dissertation to test the effects of communicated stereotypes and variations to the social categorization process on SAHMs' and WMs' intergroup communication outcomes. The results of Study One demonstrated significant main effects for SAHM and WM stereotype conditions and significant univariate effects for many of the individual stereotype conditions in regard to seven of the eight dependent variables (i.e., outgroup attitudes, contempt, envy, pity, facilitative behaviors, harmful behaviors, and intergroup anxiety). In particular, the lazy, selfish, and lost; busy and overworked, and ideal mother stereotypes elicited more negative attitudes, affect, and behaviors compared to the traditional, supermom, and overextended with no free time, and family-oriented and flexible stereotype conditions. No significant effects were obtained for willingness to communicate across SAHM or WM stereotype conditions. Study One also revealed a significant stereotype $\mathrm{x}$ group condition interaction for facilitative behaviors and intergroup anxiety when WM stereotypes were the target of the analysis. Further dissection of this interaction effect demonstrated that WMs reported more facilitative behaviors for the overextended with no free time, hardworking and determined, and supermom WM stereotype conditions; where as SAHMs reported more facilitative behaviors for the family-oriented and flexible, traditional, and non-traditional WM stereotype conditions. Additionally, WMs reported higher levels of intergroup anxiety for the supermom and family-oriented and flexible WM stereotype conditions; whereas SAHMs reported higher levels of anxiety for overextended with no free time, hardworking and determined, traditional, and non-traditional WM stereotype conditions. Finally, Study One evidenced SAHMs' strong ingroup bias by way of their highly favorable attitudes toward target SAHM mothers. 


\section{Table 12.}

Planned Contrast Results for the Representational Mediator Conditions across Dependent Variables

\begin{tabular}{|c|c|c|c|c|c|c|c|c|c|c|c|c|c|c|c|}
\hline \multirow[b]{3}{*}{ Variables } & \multicolumn{5}{|c|}{$\begin{array}{c}\text { One Group Recategorization } \\
\text { vs. } \\
\text { Two Group Categorization }\end{array}$} & \multicolumn{5}{|c|}{$\begin{array}{c}\text { Two Subgroups in One Group } \\
\text { Recategorization } \\
\text { vs. } \\
\text { Two Group Categorization }\end{array}$} & \multicolumn{5}{|c|}{$\begin{array}{c}\text { Separate Individuals Decategorization } \\
\text { vs. } \\
\text { Two Group Categorization }\end{array}$} \\
\hline & \multirow[b]{2}{*}{$M_{\text {diff }}$} & \multirow[b]{2}{*}{$\begin{array}{l}\text { Std. } \\
\text { Error }\end{array}$} & \multicolumn{3}{|c|}{ Confidence Intervals } & \multicolumn{5}{|c|}{$\begin{array}{l}\text { Confidence } \\
\text { Intervals }\end{array}$} & \multicolumn{5}{|c|}{$\begin{array}{l}\text { Confidence } \\
\text { Intervals }\end{array}$} \\
\hline & & & $p$ & $\begin{array}{l}\text { Lower } \\
\text { Bound }\end{array}$ & $\begin{array}{l}\text { Upper } \\
\text { Bound }\end{array}$ & $M_{\text {diff }}$ & $\begin{array}{l}\text { Std. } \\
\text { Error }\end{array}$ & $p$ & $\begin{array}{l}\text { Lower } \\
\text { Bound }\end{array}$ & $\begin{array}{l}\text { Upper } \\
\text { Bound }\end{array}$ & $M_{\text {diff }}$ & $\begin{array}{l}\text { Std. } \\
\text { Error }\end{array}$ & $p$ & $\begin{array}{l}\text { Lower } \\
\text { Bound }\end{array}$ & $\begin{array}{l}\text { Upper } \\
\text { Bound }\end{array}$ \\
\hline $\begin{array}{l}\text { Stereotype } \\
\text { Reliance }\end{array}$ & .06 & .12 & .62 & -.18 & .31 & .11 & .12 & .36 & -.13 & .35 & .17 & .12 & .16 & -.07 & .40 \\
\hline $\begin{array}{l}\text { Outgroup } \\
\text { Attitudes }\end{array}$ & .41 & .52 & .43 & -.60 & 1.42 & .34 & .50 & .49 & -.64 & 1.33 & -.52 & .49 & .30 & -1.49 & .46 \\
\hline $\begin{array}{l}\text { Outgroup } \\
\text { Typicality }\end{array}$ & .00 & .04 & .99 & -.08 & .08 & .08 & .04 & .04 & .00 & .16 & .12 & .04 & .00 & .04 & .20 \\
\hline Helping & -.06 & .09 & .48 & -.24 & .11 & -.05 & .09 & .53 & -.22 & .12 & -.09 & .09 & .32 & -.25 & .08 \\
\hline $\begin{array}{l}\text { Willingness to } \\
\text { Communicate }\end{array}$ & -.03 & .08 & .69 & -.19 & .13 & -.14 & .08 & .06 & -.30 & .01 & -.03 & .08 & .74 & -.18 & .12 \\
\hline
\end{tabular}




\section{Table 13.}

Descriptive Statistics for Dependent Variables across Representational Mediator Conditions

\begin{tabular}{lcccccccc}
\hline & $\begin{array}{c}\text { One Group } \\
\text { Recategorization } \\
(n=35)\end{array}$ & $\begin{array}{c}\text { Two Subgroups } \\
\text { in One } \\
\text { Recategorization } \\
(n=39)\end{array}$ & $\begin{array}{c}\text { Two Group } \\
\text { Categorization } \\
(n=41)\end{array}$ & $\begin{array}{c}\text { Separate } \\
\text { Individuals } \\
\text { Decategorization } \\
(n=39)\end{array}$ \\
\cline { 2 - 9 } Variables & $M$ & $S D$ & $M$ & $S D$ & $M$ & $S D$ & $M$ & SD \\
\hline Stereotype Reliance & 3.95 & .547 & 4.04 & .54 & 4.06 & .63 & 3.95 & .42 \\
Outgroup Attitudes & 8.37 & 2.10 & 8.44 & 2.33 & 7.56 & 2.52 & 8.21 & 2.05 \\
Outgroup Typicality & 4.22 & .54 & $4.56^{\mathrm{b}}$ & .80 & $4.73^{\mathrm{a}}$ & .91 & $4.20^{\mathrm{b}}$ & .57 \\
Helping & 2.68 & 2.69 & 2.73 & 2.82 & 2.33 & 2.03 & 2.79 & 2.43 \\
Willingness to Communicate & 5.11 & 1.19 & 5.53 & 1.07 & 5.12 & 1.22 & 5.12 & 1.13 \\
\hline
\end{tabular}

Note. The dissimilar superscripts for outgroup typicality represent mean differences revealed in the planned contrasts reported in Table 10. 
The results of Study Two revealed a nearly significant main effect for the representational mediator conditions and a significant univariate effects for three of the representational mediator conditions in regard to one of the five dependent variables (i.e., outgroup typicality). The planned contrast results demonstrated that the two subgroups in one group recategorization and separate individuals decategorization mediators obtained significantly lower reports of outgroup typically compared to the two group categorization mediator. No significant effects were obtained for stereotype reliance, outgroup attitudes, helping, or willingness to communicate across the representational mediator conditions. The theoretical and practical implications of these results are discussion in Chapter IV of this dissertation. 


\section{Chapter IV}

\section{Discussion}

The purpose of this dissertation was to demonstrate the cognitive, affective, and behavioral consequences to communicated stereotypes and transformed categorization within intergroup interactions between SAHMs and WMs. Toward achieving this goal, two independent experiments were conducted in accordance with the central premises of three foundational intergroup theories: social identity theory (SIT; Tajfel \& Turner, 1986), selfcategorization theory (SCT; J. C. Turner et al., 1987), and the common ingroup identity model (CIIM; Gaertner et al., 1993). In Study One, the effects of stereotypes on SAHMs' and WMs' outgroup attitudes, affective and behavioral responses, intergroup anxiety, and willingness to communicate were examined. Generally, the results of Study One provide support for the hypotheses by demonstrating different stereotype content elicits different reactions among mothers. In Study Two, the effects of manipulating the CIIM's representational mediators on SAHMs' and WMs' stereotype reliance, outgroup attitudes, perceptions of outgroup typicality, provision of help, and willingness to communicate were examined. Generally, the results of Study Two did not provide support for the hypotheses (with the exception of outgroup typicality) but present opportunities to continue unraveling the complexities of communicated social identities. The sections in this chapter will: (1) highlight the major findings of these two studies; (2) discuss the theoretical implications of these findings; (3) provide communicative solutions for resolving the cultural "mommy wars;" and (4) suggest directions for researchers' future exploration of intergroup conflict and interpersonal relationships among mothers.

The results of this dissertation demonstrate the powerful, negative effects of stereotyping — especially under circumstances of ingroup favoritism — in intergroup interactions 
and present opportunities for future work on group boundary transformation. Although participant and target mothers seemingly have much in common as women and mothers, the results demonstrate that between-group distinctions can be elicited with something as simple occupational status. These trends demonstrate support for SIT (Tajfel \& Turner, 1986) and SCT (J. C. Turner et al., 1987). Although prejudice is traditionally assessed between large, broad social groups such as women "versus" men (e.g., Chaudoir \& Quinn, 2010; Vonk \& OldeMonnikhof, 1998), the results demonstrate the extreme malleability of ingroup/outgroup distinctions, which creates opportunities for stereotyping, prejudice, and discrimination in any social interaction in which social identities are salient. As quickly as individuals can connect with others, be included in certain groups, and receive favorable treatment from their ingroup members, they can just as quickly be excluded and mistreated by outgroup members. This category fluidity leads to negative consequences for mothers—-lack of support, denial of resources, inferior societal positions, and low life satisfaction. In this dissertation we see these consequences manifest as negative attitudes, feelings, and behaviors toward mothers who exemplify certain stereotypes. Although these results present a grim view of mothers' relationships, they validate "mother" as a salient identity and a social group worthy of additional intergroup communication inquiry in order to continue improving mother-mother relationships. It is still premature to assert prescriptions regarding variations to the categorization process and, thus, support for the CIIM. However, there is evidence to suggest intergroup contact and minimized group membership in initial interactions can elicit productive outcomes for mothers in particular, and women, in general. Thus, the results advance theoretical and practical implications related to stereotype activation in real-world interactions, social comparison processes, group membership in initial intergroup contact, mothers' friendships, and feminism. 


\section{Stereotype Activation}

The findings demonstrate the powerful effects of stereotypes on social interactions by revealing the differently valenced outcomes elicited by different stereotype content. In particular, participant mothers who viewed the lazy, selfish, and lost; ideal mother; traditional; and supermom stereotype descriptions (and believed an online interaction was forthcoming with this particular type of mother) displayed more negative responses (e.g., negative outgroup attitudes, pity, contempt, intergroup anxiety, harmful behaviors) compared to participants who viewed many of the other stereotypes descriptions. As the lazy, selfish, and lost stereotype condition represents a negative portrayal of SAHMs - one who is unmotivated to work, care for her family, or find purpose in her life - negative intergroup outcomes were expected. However, unfavorable stereotypes were not the only ones that elicited negative reactions. Although the busy and overworked and overextended with no free time stereotype conditions received more favorable outgroup attitudes compared to other stereotype descriptions, these conditions also elicited a plethora of negative responses. These trends are consistent with the body of scholarship focused on the negative influence of stereotypes on intergroup interactions (e.g., Cuddy et al., 2007; Hummert et al., 1998; Ruble \& Zhang, 2012, November). Although stereotyping can serve practical functions in social interactions (Brown \& Capozza, 2000), stereotypes can be activated instantaneously and unconsciously by a variety of social cues and activated stereotypes can quickly elicit biases that hinder productive communication (Oakes, 2008). The results herein highlight the negative effects of overgeneralized characteristics of individuals and their social groups. Because the activation of both positive and negative stereotypes elicit negative outcomes, the present findings highlight the importance of looking beyond the valence of the stereotype. In this way, the outcomes associated with mothers' social 
identities parallel the trends seen in more heuristic contexts such as age, race/ethnicity, and religion.

According to the age stereotypes in interactions (ASI) model (Hummert, 1994; Hummert, Garstka, Ryan, \& Bonnesen, 2004), when ageist stereotypes (e.g., wrinkled skin, gray hair) are activated in intergenerational interactions, younger adults are likely to use patronizing "elderly speak" (Caporael, Lukaszewski, \& Culbertson, 1983) with older adult targets. This type of communication influences older adults to behave in stereotypical ways, only reinforcing ageist stereotypes (Hummert, 1994). A similar, disempowering trajectory might occur for mothers. As social identities can be communicated in various ways (Giles, 2012), stereotypes may be activated by mothers' physical appearance (e.g., weight, clothing), locations (e.g., child care center, her home), verbal (e.g., labels, language) and nonverbal (e.g., tone of voice, facial expressions) communication, or her children's behaviors (e.g., polite, disobedient). As in contexts in which salient ageist or racial stereotypes hinder personalized and productive intergenerational or interracial interactions (e.g., Hummert et al., 1998; Ruble \& Zhang, 2012, November), when stereotypes are salient in real-life interactions between mothers, mothers are likely to communicate in negative ways. In fact, even the ideal mother and supermom stereotype conditions, which depict extremely positive images of loving, attentive, and efficient mothers, elicit prejudice that discourage interactions and impede relational development (based on the unfavorable attitudes and high levels of intergroup anxiety and contempt these stereotypes elicited). Thus, these patterns will only perpetuate the social conflict between mothers and hinder mothers' ability to form personalized relationships with each other. As other scholars have demonstrated in other intergroup contexts (e.g., Allport, 1954; Oakes, 2008; Pettigrew, 1998), increased contact among outgroup mothers, as well as more time and motivation to 
process information about outgroup mothers during intergroup contact experiences, could minimize mothers' stereotype activation and promote more open, non-judgmental interactions among subgroups of mothers. In this sense, this dissertation makes an important contribution toward uniting and fostering interpersonal relationships among mothers.

\section{Social Comparison among Stay-at-home and Working Mothers}

The process of social comparison provides an insightful lens to interpret the effects of stereotypes between these subgroups of mothers-particularly stereotypes previously presumed to be favorable (e.g., ideal mother, supermom) or neutral (e.g., busy and overworked). Social comparison theory (Festinger, 1954), a classic social psychology theory originating from research on group dynamics and social judgment (Suls \& Wheeler, 2012), asserts that individuals determine their own personal and social worth by comparing themselves with similar others across a variety social domains (e.g., career success, money, attractiveness, intelligence, popularity). Social comparison can be directed upward to individuals perceived to be superior to the self or downward to less fortunate individuals (Festinger, 1954). Engaging in self- and other evaluation reduces uncertainty about the arbitrary standards within each domain, helps individuals recognize their strengths and abilities, and justifies individuals' future behaviors (Wood, 1989).

Social comparison theory serves as an appropriate lens through which to interpret mothers' envious responses to favorable mother prototypes (i.e., ideal mother, supermom, busy and overworked, executive of the home), and these envious responses serve as appropriate explanations for the negative outcomes elicited by these stereotype conditions. Envy involves begrudgingly admiring a target due to perceiving their outcomes (e.g., societal position, career, material possessions, social networks) as superior to one's own outcomes. Envy is considered an 
ambivalent emotion because it incorporates both positive (e.g., respect, admiration) and negative components (e.g., resentment, hostility; Cuddy et al., 2007). Although envy often leads to feelings of injustice, incompetence, or inferiority, these feelings manifest in both positive and negative ways - another reason for envy's ambivalent classification. Envy can elicit compromising behaviors as an attempt to obtain some of the target's coveted outcomes when the target is unthreatening or hostile behaviors as an attempt to deny the target future success when the target is perceived to have the ability to disrupt society with their competence and power. Under either circumstance, targets of these responses do not receive genuine support and kindness from outgroup members and would have reason to fear volatile outgroup members; thus, create tense intergroup relationships.

The findings suggest mothers' upward comparisons may be a catalyst for the cultural "mommy wars." When mothers upwardly compare themselves to mothers who display prototypical traits that align with the favorable stereotype profiles (e.g., domestic, selfless, involved, efficient, organized), they may feel jealous or resentful of the target mothers' maternal competence. In addition to harboring negative attitudes for these mother prototypes, envious responses may lead mothers to harshly criticize their own maternal competence, concluding they are incapable of meeting societal expectations of motherhood in comparison to these favorable mother prototypes. Social comparison scholarship (see Wood, 1989) suggests mothers' selfesteem and self-concept would be effected by self-assessments of their maternal competence. Results from this dissertation suggest mothers' feelings of maternal incompetence produce high levels of anxiety. Although participant mothers were willing to communicate with the target mothers in this dissertation, intergroup research has been consistently demonstrated anxiety as a deterrent to interactions and relational development with outgroup members (Stephan, 2014). 
Anxiety is often rooted in fear of the outgroup, being negatively stereotyped by the outgroup, or insecurity about one's own communication efficacy (Huang, Sedlovskaya, Ackerman, \& Bargh, 2011; Islam \& Hewstone, 1993; McKenna, Samuel-Azran, \& Sutton-Balaban, 2009). Thus, it seems as if mothers' intergroup anxiety—perhaps stemming from social comparisons and feelings of maternal incompetence - would influence mothers to avoid communication with favorable mother prototypes, or remove them from their mother subgroup altogether, which would only strengthen the division among mothers. Thus, the cultural "mommy wars" may be a direct result of mothers feeling incompetent and anxious in regard to favorable mother prototypes. Given that these favorable stereotype conditions also elicited fewer facilitative and/or more harmful behaviors - particularly from outgroup mothers — envy likely plays a large role in SAHMs' and WMs' judgmental, exclusive, and hurtful relationships. Researchers should further explore the role envy plays in mothers' interpersonal relationships.

Social comparison theory also serves as an appropriate lens through which to interpret mothers' piteous responses to favorable mother prototypes (i.e., lazy, selfish, and lost; overextended with no free time), and these piteous responses serve as appropriate explanations for the negative outcomes elicited by these stereotype conditions. Pity, another ambivalent emotion, includes both positive and negative components such as compassion and sadness (Cuddy et al., 2007). Although pity may originate as a well-intentioned response to outgroup members, it has been associated with avoidant behaviors when the target's situation appears out of the target's control (e.g., ignoring a homeless person's request for money) and overaccommodating behaviors (e.g., talking louder, slower, or simpler to older adults) when the target's situation highlights the target's incompetence (Cuddy et al., 2004). Under either circumstance, targets of these responses feel neglected and powerless. 
Mothers' downward comparisons have practical implications for the cultural "mommy wars." When mothers downwardly compare themselves to mothers who display prototypical traits that align with the unfavorable stereotype profiles (e.g., lazy, selfish, overextended, stressed, tired), they feel sympathy or despair for the target mothers' hopeless or stressful situations. Pity may lead mothers to inflate their self-evaluations, concluding their mothering and family situations are more desirable in comparison to these unfavorable mother prototypes. These biased self-perceptions create high levels of anxiety and likely influences mothers to avoid, exclude, and disassociate with these unfavorable mother prototypes. In turn, these nonfacilitative behaviors fuel the conflicts among SAHMs and WMs and prohibit opportunities to learn about mothers' characteristics that are divergent from the stereotypes.

Given the evidence presented in this dissertation, it is possible that SAHMs are more susceptible to downward comparisons and, potentially, more responsible for the cultural "mommy wars." The ingroup favoritism effect was stronger for SAHMs as they have a higher propensity to rate their ingroup members more favorably than outgroup members and more favorably than outgroup members rate themselves. These trends are consistent with Johnston and Swanson (2004) who revealed SAHMs' portrayal of employed mothers as more negative, critical, and stereotypical than WMs' portrayals of SAHM. Ingroup favoritism has been linked to positive affect toward and preferential treatment to members of one's own social groups (Allport, 1954; Hecht, 1998b). SAHMs' reported more facilitative behaviors, or preferential treatment, for WMs who display characteristics (e.g., traditional, child-focused, patient, involved with children, overbearing) that are associated with intensive mothering. Intensive mothering (i.e., the belief that mothers must be exclusively absorbed in activities that protect, nurture, and socialize children) is the dominant and traditional mothering ideology and consistently 
associated with SAHMs (Hays, 1996; D. D. Johnston \& Swanson, 2007). SAHMs' intensive mothering practices position these mothers in accordance with societal expectations of women in the family (Arendell, 2000); thus, strengthening SAHMs' group vitality. The results in this dissertation demonstrate that SAHMs are more likely than WMs to help, protect, and associate with mothers whom they perceive as similar to themselves (and, thus, part of their SAHM ingroup) and exclude, demean, or attack mothers whom they perceive as dissimilar from themselves (and, thus, part of the WM outgroup). Given their group vitality, SAHMs likely justify judging and excluding mothers who are not actively engaged in intensive mothering as a strategy to tout and encourage their superior mothering ideology to "deviant" WMs (Arendell, 2000). As Johnston and Swanson (2004) concluded that SAHMs are more likely to internalize "mother war rhetoric" (p. 497), the social conflict between SAHMs and WMs can likely be attributed to SAHMs' ingroup favoritism and downward comparisons.

\section{Hostile Media Effect}

Although this dissertation focused primarily on mothers' interactions and relationships, its impetus (i.e., the cultural "mommy wars") and implications are highly useful in the realm of media theory and scholarship. In light of the hostile media effect (Vallone, Ross, \& Lepper, 1985), heightened media attention of the "mommy wars" likely fuels SAHMs negative attitudes, emotions, and communication behaviors toward WMs. The hostile media effect explains social conflicts via viewers' tendencies to perceive media coverage as biased in favor of the viewers' outgroups and in opposition to viewers' ingroups (Vallone et al., 1985). When viewers feel like the targets of media bias they misinterpret new information in ways that justify their biased positions and sustain positive ingroup distinctiveness (Vallone et al., 1985). When individuals display high affective, compared to cognitive, issue involvement, such as in tense inter- ethnic 
and religious conflicts (e.g., Ariyanto, Hornsey, \& Gallois, 2007; Matheson \& Dursun, 2001), the hostile media effect is even stronger (Matthes, 2013).

Given that different stereotypes elicit different levels of contempt, envy, and pity for target mothers, encountering information about the "mommy wars" in the media likely accentuates mothers' - especially SAHMs'—perceptions of their intergroup conflict. Given its prominence in the media (i.e., nearly 25 million search results in Google; O'Connor, August 2013), the "mommy wars" were intentionally omitted from recruitment procedures so not to participant bias mothers' responses. Although it is possible that mentioning the SAHM/WM categories in the online questionnaire triggered participant mothers' thoughts and feelings about their groups' tense relationships, the lack of significant differences regarding group membership (with the exception of facilitative behaviors and intergroup anxiety for WM stereotype conditions), suggests participant mothers were not responding with the media's portrayal of their groups' conflict in mind. Because research on the hostile media effect is evidenced even under circumstances of objective media coverage (Vallone et al., 1985), resolutions seem unlikely for social conflicts that have a strong presences in the media, such as the "mommy wars." This bleak reality is reflected in O'Connor's (August 2013) comment, "if you read something enough times, you start to believe it," following Parents ' nationwide poll of 500 mothers in which 63\% of mothers indicated they believed the mommy wars exist, but only $29 \%$ seeing evidence of these wars in their communities.

Despite media's contribution to distorted views of mothers' intergroup conflict, scholars and mothers can work toward changing perceptions of motherhood and resolving the cultural “mommy wars.” Scholars can serve as social change agents by satisfying Harwood and Joyce's (2012) recommendation to integrate media analyses into investigations of intergroup contact. 
Researchers might replicate priming experiments (Ariyanto et al., 2007; Matheson \& Dursun, 2001) with different portrayals of the "mommy wars" phenomenon (Sisters, February 13, 2014; Toalson, March 15, 2015) in order to investigate media's role in SAHMs' and WMs' intergroup conflict. In doing so, scholars can contribute objective, empirical knowledge to the current biased social commentary on mothers and make scientific-based prescriptions for redirecting the media's powerful influence toward prosocial outcomes. In recent years, some organizations and bloggers have used the mass reach of social media to promote supportive, nonjudgmental relationships among mothers in which mothers' commonalities and their differences are celebrated (e.g., Connecticut Working Mothers' Campaign Judgement-Free Motherhood). Scholars could incorporate productive initiatives into their research in order to demonstrate the benefits to media coverage that portrays mothers in favorable ways.

Another resolution to the cultural "mommy wars" rests in the practical applications of intergroup contact scholarship. Because increased contact with outgroup members has long been evidenced as a solution to prejudice (Allport, 1954; Hewstone, 2003; Pettigrew \& Tropp, 2006), mothers themselves may be able to escape the cultural "mommy wars" by diversifying their own personal social networks. The findings of this dissertation demonstrate that the quality of intergroup interactions exerts more influence on intergroup outcomes compared to the frequency of these interactions. Thus, in addition to increasing their quantity of intergroup contact, mothers must also work to improve their communication competence in intergroup interactions. Based on the results herein, much of mothers' communication competence likely rests on how they communicate their group memberships in initial interactions.

\section{Group Membership in Initial Intergroup Contact}

This dissertation was the first study to experimentally test the variations to the social 
categorization process, as set forth by the CIIM, in a communication context. Researchers (Gaertner et al., 1999; Gómez et al., 2008) have previously manipulated the CIIM's representational mediators by forcing participant groups within a laboratory setting (e.g., teams with White or Blue T-shirts) or drawing upon naturally occurring identities (e.g., ethnic identities among high school students) retrospectively. This dissertation aimed to extend these studies by examining the ways in which group membership is communicated in social interactions. However, given that the representational mediators only elicited different perceptions of outgroup typicality (and the highest mean was reported for the two groups categorization condition), strong support for the CIIM was not obtained in this dissertation.

This lack of support for the CIIM has two potential explanations. The first explanation involves the study's recruitment procedures. The recruitment announcements emphasized the researcher's and participants' mothering identities and informed participant mothers that they would be interacting with another mother in the study. These procedures likely primed participant mothers about "mothering" at the onset of the study and, as a result, influenced them to consider themselves and the target mothers as members of one, large "mother" ingroup. Later, as the manipulation checks demonstrate (see Table 11), when participant mothers were presented with the representational mediator conditions in the online questionnaire, they had difficulty categorizing the target mothers as anything but "mothers" (i.e., the one group recategorization and two subgroups in one recategorization conditions). In the future, researchers should purposively sample their target demographic but de-emphasize their social identities and the primary focus of the study during recruitment so not to prime participants. If the current dissertation had followed this procedure, participant mothers may have been influenced by the nuanced variations to the categorization process within the four message conditions. 
The second explanation involves the complexities of intergroup communication. Not only is human communication, in general, a complex and dynamic process, predicting and interpreting the intricacies of intergroup dynamics in social interactions is a challenging endeavor (Giles, 2012). If participant mothers were already primed to conceive of themselves and target mothers as "mothers" (as discussed previously), a few category words (e.g., "stay-athome," "working," "mothers," "person") are likely insufficient for accentuating target mothers' subgroup membership to the degree that could overcome priming effects and elicit different reactions. In this sense, the way individuals communicate their social identities is likely too complicated to reproduce via a static, mediated message. Thus, it is not surprising — but also not completely discouraging - that this initial attempt at integrating communication into a social psychological theory was not flawless.

The significant differences for outgroup typicality and the means for the other outcomes (i.e., stereotype reliance, outgroup attitudes, and helping), although not significantly different, are trending in a direction that is consistent with the CIIM. Because social categorization is a fundamental human cognitive process that often occurs automatically and below consciousness (Oakes, 2008; Operario \& Fiske, 2008), categorization can elicit emotional and behavioral responses without individuals' being aware they have been influenced by this process. Thus, despite not being cognizant of the nuanced variations to the categorization process (e.g., one group recategorization, two subgroups in one group recategorization, two groups categorization, and separate individuals), it is possible that some participant mothers were influenced by the representational mediator conditions but not enough to influence variations in their responses to the target mothers. These trends reveal important implications for the salience of group membership in initial interactions and, thus, uncover productive ways for mothers to 
initiate relationships with outgroup mothers.

Because participant mothers' favorable attitudes did not differ across representational mediator conditions, the absence of extreme group membership may have influenced mothers to welcome all target mothers - even those in the two group categorization conditions - into their ingroup. This notion is consistent with research that has demonstrated, when cues related to extreme outgroup membership are made less salient, social affiliation toward outgroup members increases to the same levels afforded to ingroup members (Carr, Vitak, \& McLaughlin, 2013). In this sense, less salient group membership influences individuals to include otherwise dissimilar outgroup members into their existing ingroups or a new, common ingroup. Thus, although activated stereotypes exert negative effects on intergroup interactions, initial interactions among mothers may be more productive, and lead to future interactions, if mothers' different family/work arrangements (i.e., subgroup memberships) are not overtly communicated upon meeting for the first time.

Despite the prosocial effects on intergroup contact demonstrated through the CIIM, positive reactions to group membership may not be sustained as mothers' relationships progress and the breadth and depth of self-disclosures increase. Although self-disclosure has been linked to trust and relational quality (see Harwood \& Joyce, 2012), as mothers' learn more about each other's family arrangements, lifestyles, and decisions, ingroup/outgroup distinctions will likely become more salient. However, at low levels of intergroup anxiety and high levels of empathy and convergence (see Harwood \& Joyce, 2012), more knowledge about the outgroup can facilitate satisfying interpersonal relationships among mothers. These trends provide clear avenues for future investigations of the CIIM in which variations to mothers' categorization are experimentally assessed across a sequence of interactions in more developed relationships. 


\section{Mothers' Interpersonal Relationships in Online Contexts}

As this dissertation's initial interaction framework extends to address ongoing intergroup interactions between SAHMs and WMs, it is important to draw from the intergroup scholarship on cross-group friendships that has concluded intergroup contact within close relationships exerts powerful influence on prejudice reduction (e.g., Davies, Tropp, Aron, Pettigrew, \& Wright, 2011; Wright, Aron, McLaughlin-Volpe, \& Ropp, 1997). Participant mothers were eager to participate in a study focused on interacting with other mothers, perhaps because forming adult relationships is challenging after kids. The Comma Mommas Facebook group united $69.40 \%$ of participant mothers following the studies and these mothers are now actively engaged in daily conversations about family, motherhood, and relationships issues (e.g., family vacation ideas, sex with romantic partners, parenting advice) that are personal/intimate and shared among mothers. Although no empirical evidence regarding the content (and intergroup/interpersonal nature) of mothers' messages in the Comma Mommas Facebook group has yet been uncovered, mothers have forged connections with other mothers as they set out to do at the beginning of the studies. Further, mothers' frequent activity in the group (approximately 3-5 conversation threads per day) suggests mothers can overcome their internal struggles by forming cross-group friendships_-particularly in online contexts.

Given the many affordances of online communication (Amichai-Hamburger, 2012; Harwood et al., 2012), the Comma Mommas Facebook group may have facilitated mothers' connections over and above what could be possible in face-to-face (FtF) interactions. The four online communication affordances most applicable to the findings of this dissertation, mothers' activity in the Facebook group, and practical applications of intergroup scholarship are asynchronicity, reduced nonverbal cues, convenience, and accessibility. As asynchronicity 
affords individuals more time to process social stimuli, carefully encode and decode messages, and construct feedback (Walther, 1996), the temporal nature of online communication has the potential to reduce mothers' reliance on stereotypes and increase their accommodative communication, which, based on research of stereotypes and accommodation in other intergroup contexts (e.g., Hummert et al., 2004; Imamura, Zhang, \& Harwood, 2011; Ruble, 2011), has the potential to create more satisfying, interpersonal relationships among mothers. Because reducedcue online environments can induce interpersonal similarity and social affiliation among outgroup members (Carr et al., 2013; E. Lee, 2004), mothers' perceived similarity (evidenced by nonsignificant differences across the representational mediator conditions) could redirect mothers' ingroup favoritism toward members of a common ingroup, allowing mothers to form personalized connections with previously dissimilar outgroup mothers. Given the links between the convenient online communication and decreased social anxiety (Amichai-Hamburger \& BenArtzi, 2003; McKenna \& Bargh, 2000), and the links between reduced intergroup anxiety and reduced prejudice (Hewstone \& Swart, 2011; Islam \& Hewstone, 1993), mothers' continued contact with outgroup mothers via online environments, should minimize their intergroup anxiety and foster more positive intergroup relations in the short and long term. Finally, because mothers' participation in the Comma Mommas Facebook group provides mothers premium access to an expansive social network that include outgroup mothers, there is also potential for the extended contact hypothesis (i.e., positive intergroup attitudes result from knowing ingroup members have close relationships with outgroup members; Wright et al., 1997) to motivate mothers' relational formation online.

The aforementioned discussion of online affordances reveals two directions for future intergroup research with subgroups of mothers. Both directions involve satisfying intergroup 
exerts' (e.g., Harwood \& Joyce, 2012; Hewstone et al., 2014) calls for more ecologically valid, longitudinal intergroup research that focuses on the contact channels and content. The first way researchers can answer these calls is by observing and analyzing, across a period of time, the content of mothers' publicly documented, readily accessible, and naturally occurring online conversations in a group such as Comma Mommas. Such data would reveal mothers' authentic perceptions and messages about their social identities and intergroup conflict free of research intervention. Additionally, this research could provide evidence for the ways in which "mommy wars" are communicated, which could complement (or even discredit) the media's commentary of this phenomenon.

The second way researchers can answer previous calls is by examining the trajectory of intergroup contact. Positive intergroup contact effects evidenced in parasocial (Ortiz \& Harwood, 2007; Schiappa et al., 2005) and imagined (R. N. Turner, Crisp, \& Lambert, 2007; Vezzali, Capozza, Stathi, \& Giovannini, 2012) interactions have lead researchers (e.g., AmichaiHamburger \& McKenna, 2006; Crisp \& Turner, 2009) to advocate for a gradual progression of intergroup contact in which outgroup mothers initiate contact through indirect means (e.g., imagined, extended, vicarious) and work up to contact through direct means (e.g., FtF, rich online environments). Researchers could design their studies such that intergroup contact begins with mothers engaging in parasocial interactions with outgroup mothers via media clips, moves to mothers observing contact between members of their ingroup and members of the outgroup, and ends with mothers engaging in discussions with outgroup members via Web-based, video/text chat tools. Such data would validate the sequence of contact and uncover mechanisms that can reduce mothers' intergroup anxiety and motivate positive intergroup contact. 


\section{Mothers and Feminism}

The findings in this dissertation present great opportunities for feminist researchers and activists as there is still much work to be done to empower women in and outside of their families. Not only do stereotypes limit mothers' opportunities and societal positions by reducing them to overgeneralized traits and behaviors (e.g., "traditional," "lazy") and holding them to unrealistic feminine expectations (e.g., "ideal mother," "supermom”), they create arbitrary distinctions among subgroups of mothers. When mothers allow themselves to be distracted by unproductive debates about their differences, they divert attention away from real issues families face (e.g., affordable healthcare, gay parents' adoptive rights, the gendered wage gap, quality child care, family leave policies; Zimmerman et al., 2008). If women desire increased representation in public contexts (e.g., education, government, workplaces) in order to voice their opinions on family-related social change, women must be unified at the forefront of these changes.

Additionally, when mothers focus on their social conflict instead of celebrating, appreciating, and learning from their unique perspectives, their large social group loses vitality and becomes susceptible to outgroup denigration. Given the "irony of harmony," the trend for advantaged groups' to prefer discussions with disadvantaged group members that minimize differences and accentuate commonalities (i.e., the "colorblind" ideology; Saguy, Tausch, Dovidio, \& Pratto, 2009), mothers (and women, in general) must remain unified in order to ensure their unique worth and contributions are not discredited (or, at the very least, ignored) by non-mothers, fathers (or men in general), and policy makers. Feminist scholars conclude that, if mothers (and women) continue to devalue each other and be their own "worst enemies," they will only reinforce patriarchal prejudice and indirectly advocate for their own oppression and 
mistreatment (Ashcraft \& Pacanowsky, 1996; Lorde, 1979). Because social media has a unique ability to rally collective action around various social and political initiatives based on perceived similarity and ingroup membership (Abroms \& Craig Lefebvre, 2009; Brunsting \& Postmes, 2002; Postmes \& Brunsting, 2002), mothers' communication in online contexts such as the Comma Mommas Facebook group may foster connections strong enough to reduce sexism aimed at their female group. Further, if the Internet truly is an equalizer (Dubrovsky, Kiesler, \& Sethna, 1991), a unified "mother" voice may inspire non-mothers and fathers to join mothers' conversations and feminist initiatives. Thus, as researchers uncover ways to reduce mothers' stereotyping and social comparisons and increase mothers' inclusive and accommodative communication with outgroup mothers, they will also be working toward greater equality for women overall.

\section{Limitations}

Although the findings presented in this dissertation have theoretical and practical utility for researchers and mothers alike, there are five limitations worth mentioning. The first limitation relates to both pilot studies and the manipulations in Study Two's main experiment. As previously discussed in Chapter Three, the results of the pilot studies did not validate the experimental conditions used in the main studies. In Study One, the successful manipulation checks and the results in the main study demonstrate that participant mothers were influenced by the stereotypes as expected. Thus, the unsuccessful pilot study was, ultimately, less meaningful to Study One's design. However, in Study Two, the unsuccessful manipulation checks demonstrate more work is needed to create successful experimental conditions and fully understand how the CIIM mediators can induce various intergroup outcomes. In the future, researchers should recruit similar samples for both pilots and main studies in order to ensure 
results are not due to the outgroup homogeneity effect (Jones, Wood, \& Quattrone, 1981) and pilot studies can adequately inform experimental conditions.

The second limitation involves self-selection bias. Participant mothers' affluence and personal connections to the researchers may have influenced them to select themselves as participants and become extremely invested in this dissertation. Participant mothers likely value scientific research (and, possibly, understand the difficulty researchers experience when recruiting participants), are highly committed to mothering, or felt pressure to help another mother (who was also their family member, colleague, or friend in some cases). In addition to creating homogenous samples (discussed as the third limitation below), this self-selection bias may have influenced participant mothers to diligently recruit other participant mothers, express a strong willingness to communicate with the target mothers, and advance to the online interactions with little to no hesitation. However, despite all the circumstances that may have motivated mothers to participate and report favorable reactions to the target mother, the results demonstrate the negative effects of stereotyping among mother subgroups. Additionally, given that the effects of intergroup contact are more pronounced when participants are forced into intergroup interactions (Pettigrew \& Tropp, 2006), it is likely that the stereotype effects would have been even stronger and more significant effects for representational mediator conditions would have been uncovered if participant mothers had not participated under their own volition.

This third limitation refers to the homogenous samples. Because the "choice" surrounding maternal employment is strongly rooted in privilege (e.g., White, middle-class, educated, married), minority group mothers' (e.g., Black, Latina, poor, single) employment “choices" are often based on family tradition, immigration, and survival (Dillaway \& Pare, 2008; Giele, 2008). The divisions among minority mothers' family/work roles are blurred and, thus, 
cannot be easily dichotomized into the "staying home" and "working" subgroups (Arendell, 2000). Indeed, minority mothers have distinct discourses to convey "working motherhood" (P. M. Buzzanell, Waymer, Paz Tagle, \& Liu, 2007). It is possible that the stereotypes and messages would have elicited different levels of outgroup attitudes, feelings, and behaviors if a more diverse sample (in terms of marital status, race, education level, and household income) had participated in the studies herein. Future researchers should consider recruiting mothers from neighborhoods and organizations that serve low-income families and minority populations (e.g., government-funded early education programs, county assistance programs, urban public schools).

Despite a non-diverse sample, the findings provide consistency for previous research on the SAHM and WM stereotypes, the cultural "mommy wars," and intergroup communication scholarship. The stereotype conditions used in this dissertation were derived from stereotypes that highly educated and affluent samples identified (Odenweller \& Rittenour, 2014, November; 2015, April). Additionally, the media's portrayal of "mothering" is derived from an image of families with "white, middle-class, heterosexual couples" (Zimmerman et al., 2008, p. 209). Intergroup communication scholarship on stereotypes progresses such that stereotypes are identified (e.g., Hummert et al., 1994; Ruble \& Zhang, 2013) and, then, tested for their effects on social interaction (e.g., Hummert et al., 1998; Ruble \& Zhang, 2012, November). Thus, this dissertation demonstrates consistent patterns for the specific stereotypes that were the focus of this investigation, in particular, and broader intergroup scholarship, in general.

The fourth limitation refers to participant mothers' potential knowledge of the deceptive study design. Because participant mothers were not asked to disclose this information, it is possible that some participant mothers completed the online questionnaire fully knowing the 
target mother was not a real person. Under these circumstances, the authenticity of participant mothers' responses may be questioned. However, given that the majority of participants indicated that were willing to communicate with the target mother and clicked through the final page of the questionnaire to begin their online interactions, it seems unlikely that many participants were not responding with the forthcoming online interactions in mind.

The fifth limitation involves the online questionnaires. As previously discussed in Chapter Two, the online version of the Feeling Thermometer presented a challenge during data analysis. The decision to replace this instrument's missing values with the scale mean may have improperly influenced the results. Researchers who utilize the Feeling Thermometer in their future online questionnaires should provide specific instructions to participants about their responses on this instrument and remove missing values (versus replace them). Additionally, the placement of the demographic questions at the front of the online questionnaires may have influenced the main studies' attrition rates. Researchers should place the demographic questions at the end of their questionnaires in order to prevent attrition or ensure pertinent study information is collected before participants exit the study.

\section{Conclusion}

This goal of this dissertation was to experimentally test foundational intergroup communication theories in a new intergroup context of SAHMs and WMs and uncover communicative solutions to resolving the cultural "mommy wars." The results of Study One reveal the powerful effects of stereotypes on mothers' outgroup attitudes, affective and behavioral responses, and intergroup anxiety. The results of Study Two reveal the complexities of communicating social categorization and present opportunities for future investigations of the CIIM. Taken together, this dissertation demonstrates that rigorous theory-driven, empirical 
investigations of socially significant phenomena can ignite social change efforts. Future research, media coverage, and activism focused on celebrating mothers' commonalities and differences will foster more personalized relationships between subgroups of mothers and work toward greater equality for all women. 


\section{References}

Abrams, J. R., Eveland Jr, W. P., \& Giles, H. (2003). The effects of television on group vitality: Can television empower nondominant groups? In P. J. Kalbfleisch (Ed.), Communication Yearbook (Vol. 27, pp. 193-219). Mahwah, NJ: Lawrence Erlbaum.

Abroms, L. C., \& Craig Lefebvre, R. (2009). Obama's wired campaign: Lessons for public health communication. Journal of Health Communication, 14, 415-423. doi:

$10.1080 / 10810730903033000$

Allport, G. W. (1954). The nature of prejudice. New York, NY: Perseus Books.

Alwin, D. F. (1997). Feeling Thermometers versus 7-point scales. Sociological Methods \& Research, 25, 318-340.

Amichai-Hamburger, Y. (2012). Reducing intergroup conflict in the digital age. In H. Giles (Ed.), The Handbook of Intergroup Communication (pp. 181-193). New York, NY: Routledge.

Amichai-Hamburger, Y., \& Ben-Artzi, E. (2003). Loneliness and Internet use. Computers in Human Behavior, 19, 71-80. doi: 10.1016/S0747-5632(02)00014-6

Amichai-Hamburger, Y., \& McKenna, K. Y. A. (2006). The contact hypothesis reconsidered: Interacting via the Internet. Journal of Computer-Mediated Communication, 11, 825-843. doi: 10.1111/j.1083-6101.2006.00037.x

Anderson, K., Harwood, J., \& Hummert, M. L. (2005). The grandparent-grandchild relationship: Implications for models of intergenerational communication. Human Communication Research, 31, 268-294. doi: 10.1093/hcr/31.2.268 
Arendell, T. (2000). Conceiving and investigating motherhood: The decade's scholarship. Journal of Marriage and the Family, 62, 1192-1207. doi: 10.1111/j.17413737.2000.01192.x

Ariyanto, A., Hornsey, M. J., \& Gallois, C. (2007). Group Allegiances and Perceptions of Media Bias: Taking Into Account Both the Perceiver and the Source. Group Processes \& Intergroup Relations, 10(2), 266-279. doi: 10.1177/1368430207074733

Aronson, E. (1992). Causes of prejudice. In R. M. Baird \& S. E. Rosenbaum (Eds.), Bigotry, prejudice and hatred: Definitions, causes \& solutions. (pp. 111-124). Amherst, NY: Prometheus Books.

Ashcraft, K. L., \& Pacanowsky, M. E. (1996). 'A woman's worst enemy': Reflections on a narrative of organizational life and female identity. Journal of Applied Communication Research, 24, 217-239.

Barlow, F. K., Louis, W. R., \& Hewstone, M. (2009). Rejected! Cognitions of rejection and intergroup anxiety as mediators of the impact of cross-group friendships on prejudice. British Journal of Social Psychology, 48, 389-405. doi: 10.348/014466608X387089

Becker, J. C., \& Asbrock, F. (2012). What triggers helping versus harming of ambivalent groups? Effects of the relative salience of warmth versus competence. Journal of Experimental Social Psychology, 48, 19-27. doi: 10.1016/j.jesp.2011.06.015

Berger, E. M. (2013). Happy working mothers? Investigating the effect of maternal employment on life satisfaction. Economica, 80, 23-43. doi: 10.1111/j.1468-0335.2012.00932.x

Bianchi, S. M., \& Robinson, J. (1997). What did you do today? Children's use of time, family composition, and the acquisition of social capital. Journal of Marriage and Family, 59, $332-344$. 
Binder, J., Zagefka, H., Brown, R., Funke, F., Kessler, T., Mummendey, A., . . Leyens, J.-P. (2009). Does contact reduce prejudice or does prejudice reduce contact? A longitudinal test of the contact hypothesis among majority and minority groups in three european countries. Journal of Personality and Social Psychology, 96(4), 843-856. doi:

$10.1037 / \mathrm{a} 0013470$

Bizman, A., \& Yinon, Y. (2000). Perceptions of dual identity and separate groups among secular and religious Israeli Jews. Journal of Social Psychology, 140, 589-596. doi: $10.1080 / 00224540009600498$

Bizman, A., \& Yinon, Y. (2001). Intergroup and interpersonal threats as determinants of prejudice: The moderating role of in-group identification. Basic \& Applied Social Psychology, 23, 191-196. doi: 10.1207/153248301750433669

Brescoll, V. L., \& Uhlmann, E. L. (2005). Attitudes toward traditional and nontraditional parents. Psychology of Women Quarterly, 29, 436-445.

Bridges, J. S., Etaugh, C., \& Barnes-Farrell, J. (2002). Trait judgments of stay-at-home and employed parents: A function of social role and/or shifting standards? Psychology of Women Quarterly, 26, 140-150.

Brittian, A. S., Kim, S. Y., Armenta, B. E., Lee, R. M., Umaña-Taylor, A. J., Schwartz, S. J., . . Hudson, M. L. (2015). Do dimensions of ethnic identity mediate the association between perceived ethnic group discrimination and depressive symptoms? Cultural Diversity and Ethnic Minority Psychology, 21, 41-53. doi: 10.1037/a0037531

Brown, R., \& Capozza, D. (2000). Social identity theory in retrospect and prospect. In R. Brown \& D. Capozza (Eds.), Social identity processes (pp. vii-xv). London, England: Sage. 
Brown, R., Vivian, J., \& Hewstone, M. (1999). Changing attitudes through intergroup contact: The effects of group membership salience. European Journal of Social Psychology, 29, 741-764.

Brunsting, S., \& Postmes, T. (2002). Social movement participation in the digital age: Predicting offline and online collective action. Small Group Research, 33, 525-554. doi: $10.1177 / 104649602237169$

Bureau, U. S. C. (2014). America's families and living arrangements. Retrieved from: https://www.census.gov/hhes/families/.

Buzzanell, P., Meisenbach, R., Remke, R., Liu, M., Bowers, V., \& Conn, C. (2005). The good working mother: Managerial women's sensemaking and feelings about work-family issues. Communication Studies, 56, 261-285. doi: 10.1080/10510970500181389

Buzzanell, P. M., Waymer, D., Paz Tagle, M., \& Liu, M. (2007). Different transitions into working motherhood: Discourses of Asian, Hispanic, and African American women. Journal of Family Communication, 7, 195-220. doi: 10.1080/15267430701221644

Caporael, L. R., Lukaszewski, M. P., \& Culbertson, G. H. (1983). Secondary baby talk: Judgments by institutionalized elderly and their caregivers. Journal of Personality and Social Psychology, 44, 746-754. doi: 10.1037/0022-3514.44.4.746

Carr, C. T., Vitak, J., \& McLaughlin, C. (2013). Strength of social cues in online impression formation: Expanding SIDE research. Communication Research, 40(2), 261-281. doi: $10.1177 / 0093650211430687$

Chatterji, P., Markowitz, S., \& Brooks-Gunn, J. (2013). Effects of early maternal employment on maternal health and well-being. Journal of Population Economics, 26, 285-301. doi: $10.1007 / \mathrm{s} 00148-012-0437-5$ 
Chaudoir, S., \& Quinn, D. (2010). Bystander sexism in the intergroup context: The impact of cat-calls on women's reactions towards men. Sex Roles, 62, 623-634. doi: $10.1007 / \mathrm{s} 11199-009-9735-0$

Cohn, D., Livingston, G., \& Wang, W. (2014). After decades of decline, a rise in stay-at-home mothers. Washington, DC: Pew Research Center's Social \& Demographic Trends.

Colaner, C. W., \& Giles, S. M. (2008). The baby blanket or the briefcase: The impact of Evangelical gender role ideologies on career and mothering aspirations of female Evangelical college students. Sex Roles, 58, 526-534. doi: 10.1007/s11199-007-9352-8

Costello, A. B., \& Osborne, J. W. (2005). Best practices in exploratory factor analysis: Four recommendations for getting the most from your analysis. Practical Assessment, Research, \& Evaluation, 10, 1-9. doi: http://pareonline.net/getvn.asp?v=10\&n=7

Craig, M. A., \& Richeson, J. A. (2012). Coalition or derogation? How perceived discrimination influences intraminority intergroup relations. Journal of Personality and Social Psychology, 102, 759-777. doi: 10.1037/a0026481

Crawford, V. M., \& Valsiner, J. (2002). Phenomenon, methodology and interpretation in the study of meaning: American women constructing themselves as mothers and career workers. Psychology and Developing Societies, 14, 91-130. doi: $10.1177 / 097133360201400106$

Crisp, R. J., Stone, C. H., \& Hall, N. R. (2006). Recategorization and subgroup identification: Predicting and preventing threats from common ingroups. Personality \& Social Psychology Bulletin, 32, 230-243. doi: 10.1177/0146167205280908 
Crisp, R. J., \& Turner, R. N. (2009). Can imagined interactions produce positive perceptions?: Reducing prejudice through simulated social contact. American Psychologist, 64, 231240. doi: $10.1037 / \mathrm{a} 0014718$

Crittenden, A. (2001). The price of motherhood: Why the most important job in the world is still the least valued. New York, NY: Metropolitan Books.

Crocker, J., \& Luhtanen, R. (1990). Collective self-esteem and ingroup bias. Journal of Personality and Social Psychology, 58, 60-67. doi: 10.1037/0022-3514.58.1.60

Cuddy, A. J. C., Fiske, S. T., \& Glick, P. (2004). When professionals become mothers, warmth doesn't cut the ice. Journal of Social Issues, 60, 701-718. doi: 10.1111/j.00224537.2004.00381.x

Cuddy, A. J. C., Fiske, S. T., \& Glick, P. (2007). The BIAS map: Behaviors from intergroup affect and stereotypes. Journal of Personality and Social Psychology, 92, 631-648. doi: $10.1037 / 0022-3514.92 .4 .631$

Cuddy, A. J. C., Fiske, S. T., Kwan, V. S. Y., Glick, P., Demoulin, S., Leyens, J.-P., . . Z Ziegler, R. (2009). Stereotype content model across cultures: Towards universal similarities and some differences. British Journal of Social Psychology, 48, 1-33. doi: $10.1348 / 014466608 \times 314935$

Cuddy, A. J. C., Norton, M. I., \& Fiske, S. T. (2005). This old stereotype: The pervasiveness and persistence of the elderly stereotype. Journal of Social Issues, 61, 267-285. doi: 10.1111/j.1540-4560.2005.00405.x

Davies, K., Tropp, L. R., Aron, A., Pettigrew, T. F., \& Wright, S. C. (2011). Cross-group friendships and intergroup attitudes: A meta-analytic review. Personality \& Social Psychology Review, 15(4), 332-351. doi: 10.1177/1088868311411103 
Denis, A., Ponsin, M., \& Callahan, S. (2012). The relationship between maternal self-esteem, maternal competence, infant temperament and post-partum blues. Journal of Reproductive \& Infant Psychology, 30, 388-397. doi: 10.1080/02646838.2012.718751

Dillaway, H., \& Pare, E. (2008). Locating mothers: How cultural debates about stay-at-home versus working mothers define women and home. Journal of Family Issues, 29, 437-464. doi: 10.1177/0192513X07310309

Douglas, S. J., \& Michaels, M. W. (February 2000). The mommy wars: How the media turned motherhood into a catfight. Ms. Magazine. Retrieved from: http://www.msmagazine.com/feb00/mommywars1.asp

Dovidio, J. F., Allen, J. L., \& Schroeder, D. A. (1990). Specificity of empathy-induced helping: Evidence for altruistic motivation. Journal of Personality and Social Psychology, 59, 249-260. doi: 10.1037/0022-3514.59.2.249

Dovidio, J. F., Gaertner, S. L., Hodson, G., Houlette, M. A., \& Johnson, K. M. (2005). Social inclusion and exclusion: Recategorization and the perception of intergroup boundaries. In D. Abrams, M. A. Hogg, \& J. M. Marques (Eds.), The social psychology of inclusion and exclusion (pp. 245-264). New York, NY: Psychology Press.

Dovidio, J. F., Gaertner, S. L., \& Validzic, A. (1998). Intergroup bias: Status, differentiation, and a common in-group identity. Journal of Personality and Social Psychology, 75, 109-120. doi: 10.1037/0022-3514.75.1.109

Dovidio, J. F., Gaertner, S. L., Validzic, A., Matoka, K., Johnson, B., \& Frazier, S. (1997). Extending the benefits of recategorization: Evaluations, self-disclosure, and helping. Journal of Experimental Social Psychology, 33, 401-420. 
Dubrovsky, V. J., Kiesler, S., \& Sethna, B. N. (1991). The equalization phenomenon: Status effects in computer-mediated and face-to-face decision-making groups. HumanComputer Interaction, 6, 119-146. doi: 10.1207/s15327051hci0602_2

Duckworth, J. D., \& Buzzanell, P. M. (2009). Constructing work-life balance and fatherhood: Men's framing of the meanings of both work and family. Communication Studies, 60, 558-573. doi: 10.1080/10510970903260392

Dunn, M. G., Rochlen, A. B., \& O'Brien, K. M. (2013). Employee, mother, and partner: An exploratory investigation of working women with stay-at-home fathers. Journal of Career Development, 40, 3-22.

Eagly, A. H., \& Chaiken, S. (1993). The psychology of attitudes. Orlando, FL: Harcourt Brace Jovanovich College.

Feder, D. (March 13, 2006, March). Feminists to women: Shut up and do as you're told. Human Events.

Festinger, L. (1954). A theory of social comparison processes. Human Relations, 7, 117-140. doi: $10.1177 / 001872675400700202$

Fiske, S. T., Cuddy, A. J. C., \& Glick, P. (2007). Universal dimensions of social cognition: Warmth and competence. Trends in Cognitive Sciences, 11, 77-83. doi: 10.1016/j.tics.2006.11.005

Fiske, S. T., Cuddy, A. J. C., Glick, P., \& Xu, J. (2002). A model of (often mixed) stereotype content: Competence and warmth respectively follow from perceived status and competition. Journal of Personality and Social Psychology, 82, 878-902. doi: $10.1037 / 0022-3514.82 .6 .878$ 
Gaertner, S. L., Dovidio, J. F., Anastasio, P. A., Bachman, B. A., \& Rust, M. C. (1993). The common ingroup identity model: Recategorization and the reduction of intergroup bias. European Review of Social Psychology, 4, 1-26. doi: 10.1080/14792779343000004

Gaertner, S. L., Dovidio, J. F., Rust, M. C., Nier, J. A., Banker, B. S., Ward, C. M., . . Houlette, M. (1999). Reducing intergroup bias: Elements of intergroup cooperation. Journal of Personality and Social Psychology, 76, 388-402. doi: 10.1037/0022-3514.76.3.388

Gaertner, S. L., Mann, J., Murrell, A., \& Dovidio, J. F. (1989). Reducing intergroup bias: The benefits of recategorization. Journal of Personality and Social Psychology, 57, 239-249. doi: 10.1037/0022-3514.57.2.239

Gaertner, S. L., Mann, J. A., Dovidio, J. F., Murrell, A. J., \& Pomare, M. (1990). How does cooperation reduce intergroup bias? Journal of Personality and Social Psychology, 59, 692-704. doi: 10.1037/0022-3514.59.4.692

Gaertner, S. L., Rust, M. C., Dovidio, J. F., Bachman, B. A., \& Anastasio, P. A. (1994). The contact hypothesis: The role of a common ingroup identity on reducing intergroup bias. Small Group Research, 25, 224-249.

Giele, J. Z. (2008). Homemaker or career woman: Life course factors and racial influences among middle class Americans. Journal of Comparative Family Studies, 39, 392-411.

Giles, H. (2012). Principles of intergroup communication. In H. Giles (Ed.), The Handbook of Intergroup Communication (pp. 3-18). New York, NY: Routledge.

Glenn, E. N. (1994). Social constructions of mothering: A thematic overview. In E. N. Glenn, G. Chang, \& L. R. Forcey (Eds.), Mothering: Ideology, experience, and agency (pp. 1-32). New York, NY: Routledge. 
Gómez, Á., Dovidio, J. F., Huici, C., Gaertner, S. L., \& Cuadrado, I. (2008). The other side of we: When outgroup members express common identity. Personality and Social Psychology Bulletin, 34, 1613-1626.

Gorman, K. A., \& Fritzsche, B. A. (2002). The good-mother stereotype: Stay at home (or wish that you did!). Journal of Applied Social Psychology, 32(10), 2190-2201. doi: 10.1111/j.1559-1816.2002.tb02069.x

Graham, C. W., Sorell, G. T., \& Montgomery, M. J. (2004). Role-related identity structure in adult women. Identity: An International Journal of Theory and Research, 4, 251-271.

Haas, J. (2012). Hate speech and stereotypic talk. In H. Giles (Ed.), The Handbook of Intergroup Communication (pp. 128-140). New York, NY: Routledge.

Hains, S. C., Hogg, M. A., \& Duck, J. M. (1997). Self-categorization and leadership: Effects of group prototypicality and leader stereotypicality. Personality and Social Psychology Bulletin, 23, 1087-1099. doi: 10.1177/01461672972310009

Hajek, C., Abrams, J. R., \& Murachver, T. (2008). Female, straight, male, gay, and world betwixt and between: An intergroup approach to sexual and gender identities. In J. Harwood \& H. Giles (Eds.), Intergroup communication: Multiple perspectives (Vol. 2, pp. 43-64). New York, NY: Peter Lang.

Halpern, D. F. (2005). Psychology at the intersection of work and family: Recommendations for employers, working families, and policymakers. American Psychologist, 60, 397-409. doi: 10.1037/0003-066X.60.5.397

Harwood, J. (2010). The contact space: A novel framework for intergroup contact research. Journal of Language and Social Psychology, 29, 147-177. doi: $10.1177 / 0261927 X 09359520$ 
Harwood, J., Giles, H., \& Bourhis, R. Y. (1994). The genesis of vitality theory: Historical patterns and discoursal dimensions. International Journal of the Sociology of Language, 108, 167-206.

Harwood, J., Giles, H., \& Palomares, N. A. (2008). Intergroup theory and communication processes. In J. Harwood \& H. Giles (Eds.), Intergroup communication: Multiple perspectives (2nd ed., pp. 1-17). New York, NY: Peter Lang.

Harwood, J., Hewstone, M., Amichai-Hamburger, Y., \& Tausch, N. (2012). An integration of social psychological and communication perspectives. Communication Yearbook, 36, 55102.

Harwood, J., \& Joyce, N. (2012). Intergroup contact and communication. In H. Giles (Ed.), The Handbook of Intergroup Communication (pp. 167-180). New York, NY: Routledge.

Harwood, J., Raman, P., \& Hewstone, M. (2006). The family and communication dynamics of group salience. Journal of Family Communication, 6, 181-200. doi: 10.1207/s15327698jfc0603_2

Harwood, J., \& Roy, A. (1999). The portrayal of older adults in indian and U.S. magazine advertisements. Howard Journal of Communications, 10, 269-280. doi: $10.1080 / 106461799246753$

Harwood, J., \& Vincze, L. (2012). Undermining stereotypes of linguistic groups through mediated intergroup contact. Journal of Language \& Social Psychology, 31, 157-175. doi: $10.1177 / 0261927 X 12438358$

Hays, S. (1996). The cultural contradictions of motherhood. New Haven, CT: Yale University. Hecht, M. L. (1998a). (In)Conclusion. In M. L. Hecht (Ed.), Communicating prejudice (pp. 337338). Thousand Oaks, CA: Sage. 
Hecht, M. L. (1998b). Introduction. In M. L. Hecht (Ed.), Communicating prejudice (pp. 3-23). United States of America: Sage Publications.

Heisler, J. M., \& Ellis, J. B. (2008). Motherhood and the construction of "mommy identity": Messages about motherhood and face negotiation. . Communication Quarterly, 56, 445467. doi: 10.1080/01463370802448246

Hewstone, M. (2003). Intergroup contact: Panacea for prejudice? The Psychologist, 16, 352-355.

Hewstone, M., \& Brown, R. (1986). Contact is not enough: An intergroup perspective on the 'contact hypothesis'. In M. Hewstone \& R. Brown (Eds.), Contact and conflict in intergroup encounters (pp. 1-44). Cambridge, MA: Basil Blackwell.

Hewstone, M., Lolliot, S., Swart, H., Myers, E., Voci, A., Al Ramiah, A., \& Cairns, E. (2014). Intergroup contact and intergroup conflict. Peace and Conflict: Journal of Peace Psychology, 20, 39-53. doi: 10.1037/a0035582

Hewstone, M., \& Swart, H. (2011). Fifty-odd years of inter-group contact: From hypothesis to integrated theory. British Journal of Social Psychology, 50, 374-386. doi: 10.1111/j.2044-8309.2011.02047.x

Huang, J. Y., Sedlovskaya, A., Ackerman, J. M., \& Bargh, J. A. (2011). Immunizing against prejudice: Effects of disease protection on attitudes toward out-groups. Psychological Science, 22, 1550-1556. doi: 10.1177/0956797611417261

Hummert, M. L. (1994). Stereotypes of the elderly and patronizing speech. In M. L. Hummert, J. M. Wiemann, J. F. Nussbaum, M. L. Hummert, J. M. Wiemann, \& J. F. Nussbaum (Eds.), Interpersonal communication in older adulthood: Interdisciplinary theory and research. (pp. 162-184). Thousand Oaks, CA: Sage Publications, Inc. 
Hummert, M. L., Garstka, T. A., Ryan, E. B., \& Bonnesen, J. L. (2004). The role of age stereotypes in interpersonal communication. In J. F. Nussbaum, J. Coupland, J. F. Nussbaum, \& J. Coupland (Eds.), Handbook of communication and aging research (Vol. 2nd, pp. 91-114). Mahwah, NJ: Lawrence Erlbaum Associates Publishers.

Hummert, M. L., Garstka, T. A., Shaner, J. L., \& Strahm, S. (1994). Stereotypes of the elderly held by young, middle-aged, and elderly adults. Journal of Gerontology, 49, 240-249.

Hummert, M. L., \& Shaner, J. L. (1994). Patronizing speech to the elderly as a function of stereotyping. Communication Studies, 45, 145-158.

Hummert, M. L., Shaner, J. L., Garstka, T. A., \& Henry, C. (1998). Communication with older adults: The influence of age stereotypes, context and communicator age. Human Communication Research, 25, 124-151. doi: 10.1111/j.1468-2958.1998.tb00439.x

Imamura, M., Zhang, Y. B., \& Harwood, J. (2011). Japanese sojourners' attitudes toward Americans: Exploring the influences of communication accommodation, linguistic competence, and relational solidarity in intergroup contact. Journal of Asian Pacific Communication, 21, 115-132.

Islam, M. R., \& Hewstone, M. (1993). Dimensions of contact as predictors of intergroup anxiety, perceived out-group variability, and out-group attitude: An integrative model. Personality and Social Psychology Bulletin, 19, 700-710. doi: 10.1177/0146167293196005

Jetten, J., Spears, R., \& Antony S. R. Manstead, R. (1997). Distinctiveness threat and prototypicality: combined effects on intergroup discrimination and collective self-esteem. European Journal of Social Psychology, 27, 635-657.

Johnston, D. D., \& Swanson, D. H. (2004). Moms hating moms: The internalization of mother war rhetoric. Sex Roles, 51, 497-509. doi: 10.1007/s11199-004-5460-х 
Johnston, D. D., \& Swanson, D. H. (2007). Cognitive acrobatics in the construction of workermother identity. Sex Roles, 57(5-6), 447-459. doi: 10.1007/s11199-007-9267-4

Johnston, L., \& Hewstone, M. (1992). Cognitive models of stereotype change: III. Subtyping and the perceived typicality of disconfirming group members. Journal of Experimental Social Psychology, 28, 360-386.

Jones, E. E., Wood, G. C., \& Quattrone, G. A. (1981). Perceived variability of personal characteristics in in-groups and out-groups: The role of knowledge and evaluation. Personality \& Social Psychology Bulletin, 7, 523-528. doi: 10.1177/014616728173024

Katz, D., \& Braly, K. (1933). Racial stereotypes of one hundred college students. The Journal of Abnormal and Social Psychology, 28, 280-290. doi: 10.1037/h0074049

Kim, J., \& Wickrama, K. A. S. (2014). Mothers' working status and infant development: Mediational processes. Journal of Family Issues, 35, 1473-1496. doi: $10.1177 / 0192513 \times 13496414$

Koropeckyj-Cox, T., Romano, V. R., \& Moras, A. (2007). Through the Lenses of Gender, Race, and Class: Students' Perceptions of Childless/Childfree Individuals and Couples. Sex Roles, 56(7/8), 415-428. doi: 10.1007/s11199-006-9172-2

LaBelle, S., Booth-Butterfield, M., \& Rittenour, C. E. (2013). Attitudes toward profoundly hearing impaired and deaf individuals: Links with intergroup anxiety, social dominance orientation, and contact. Western Journal of Communication, 77(4), 489-506. doi: $10.1080 / 10570314.2013 .779017$

Lamb, M. E. (2010). The role of the father in child development (5th ed.). Hoboken, NJ: John Wiley. 
Laughlin, L. (2011). Maternity leave and employment patterns: 2006-2008 (P70-128). Washington, D.C.: Retrieved from http://www.census.gov/population/www/socdemo/fertility.html.

Lee, E. (2004). Effects of visual representation on social influence in computer-mediated communication experimental tests of the social identity model of deindividuation effects. Human Communication Research, 30, 234-259.

Lee, S. (2005). Judgment of ingroups and outgroups in intra- and intercultural negotiation: The role of interdependent self-construal in judgment timing. Group Decision \& Negotiation, 14, 43-62. doi: 10.1007/s10726-005-3875-6

Lippmann, W. (1922). Public opinion. New York, NY: Harcourt Brace Jovanovich.

Liu, C.-C., Chen, Y.-C., Yeh, Y.-P., \& Hsieh, Y.-S. (2012). Effects of maternal confidence and competence on maternal parenting stress in newborn care. Journal of Advanced Nursing, 68, 908-918. doi: 10.1111/j.1365-2648.2011.05796.x

Lorde, A. (1979). The master's tools will never dismantle the master's house. Paper presented at the Second Sex Conference, New York, NY.

Mackie, D. M., Devos, T., \& Smith, E. R. (2000). Intergroup emotions: Explaining offensive action tendencies in an intergroup context. Journal of Personality and Social Psychology, 79, 602-616. doi: 10.1037/0022-3514.79.4.602

Matheson, K., \& Dursun, S. (2001). Social Identity Precursors to the Hostile Media Phenomenon: Partisan Perceptions of Coverage of the Bosnian Conflict. Group Processes \& Intergroup Relations, 4(2), 116. 
Matthes, J. (2013). The affective underpinnings of hostile media perceptions: Exploring the distinct effects of affective and cognitive involvement. Communication Research, 40, 360-387. doi: 10.1177/0093650211420255

McCann, R. M., Dailey, R. M., Giles, H., \& Ota, H. (2005). Beliefs about intergenerational communication across the lifespan: Middle age and the roles of age stereotyping and respect norms. Communication Studies, 56, 293-311. doi: 10.1080/10510970500319286

McKenna, K. Y. A., \& Bargh, J. A. (2000). Plan 9 from cyberspace: The implications of the Internet for personality and social psychology. Personality and Social Psychology Review, 4, 57-75. doi: 10.1207/S15327957PSPR0401_6

McKenna, K. Y. A., Samuel-Azran, T., \& Sutton-Balaban, N. (2009). Virtual meetings in the Middle East: Implementing the contact hypothesis on the Internet. Israel Journal of Conflict Resolution, 1, 63-86.

Medved, C. E. (2007). Investigating family labor in communication studies: Threading across historical and contemporary discourses. Journal of Family Communication, 7, 225-243. doi: $10.1080 / 15267430701392172$

Medved, C. E. (2009). Constructing breadwinning-mother identities: Moral, personal, and political positioning. Women's Studies Quarterly, 37, 140-156. doi: 10.1353/wsq.0.0194

Miller, N., \& Brewer, M. B. (1986). Categorization effects on ingroup and outgroup perception. In J. F. Dovidio \& S. L. Gaertner (Eds.), Prejudice, discrimination, and racism (pp. 209230). San Diego, CA: Academic Press.

Mills, B. C., \& Stevens, A. (1985). Employed and nonemployed mothers: Differences in parental child rearing practices. Early Child Development and Care, 22, 181-194. 
Mulac, A., Giles, H., Bradac, J. J., \& Palomares, N. A. (2013). The gender-linked language effect: An empirical test of a general process model. Language Sciences, 38, 22-31. doi: 10.1016/j.langsci.2012.12.004

Nielson. (2012). Infographic: The digital lives of American moms. Newsire. from http://www.nielsen.com/us/en/insights/news/2012/digital-lives-of-american-moms.html

O'Connor, G. (August 2013). Do Mommy Wars Exist? Parents. Retrieved from: http://www.parents.com/parenting/moms/do-mommy-wars-exist/

O'Reilly, A. (1996). "Ain't that love?": Antiracism and racial constructions of motherhood. In M. T. Reddy (Ed.), Everyday acts against racism: Raising children in a multiracial world (pp. 88-98). Seattle, WA: Seal Press.

Oakes, P. J. (2008). The root of all evil in intergroup relations? Unearthing the categorization process. In R. Brown \& S. L. Gaertner (Eds.), Blackwell handbook of social psychology: Intergroup processes (3rd ed., pp. 3-21). Oxford, England: Blackwell.

Oakes, P. J., Haslam, S. A., \& Turner, J. C. (1994). Stereotyping and social reality. Oxford, England: Blackwell.

Odenweller, K. G., \& Rittenour, C. E. (2014a). Attitudes and stereotypes of stay-at-home and working mothers. Manuscript submitted for publication.

Odenweller, K. G., \& Rittenour, C. E. (2014b). A social identity perspective of the cultural “mommy wars” between stay-at-home and working mothers West Virginia University, Morgantown, WV. Unpublished manuscript.

Odenweller, K. G., \& Rittenour, C. E. (2014, November). Stereotypes of stay-at-home and working mothers. Paper presented at the National Communication Association, Chicago, IL. 
Odenweller, K. G., \& Rittenour, C. E. (2015, April). The prevalence and valence of stay-at-home and working mothers' stereotypes. Paper presented at the annual meeting of the Eastern Communication Association, Philadelphia, PA.

Operario, D., \& Fiske, S. T. (2008). Stereotypes: Content, structures, processes, and context. In R. Brown \& S. L. Gaertner (Eds.), Blackwell handbook of social psychology: Intergroup processes (3rd ed., pp. 22-44). Oxford, England: Blackwell.

Ortiz, M., \& Harwood, J. (2007). A social cognitive theory approach to the effects of mediated intergroup contact on intergroup attitudes. Journal of Broadcasting \& Electronic Media, 51, 615-631. doi: 10.1080/08838150701626487

Owens, J., \& Massey, D. S. (2011). Stereotype threat and college academic performance: A latent variables approach. Social Science Research, 40, 150-166.

Penner, L. A., Dovidio, J. F., Piliavin, J. A., \& Schroeder, D. A. (2005). Prosocial behavior: Multilevel perspectives. Annual Review of Psychology, 56, 365-392. doi: 10.1146/annurev.psych.56.091103.070141

Penner, L. A., Gaertner, S. L., Dovidio, J. F., Hagiwara, N., Porcerelli, J., Markova, T., \& Albrecht, T. L. (2013). A social psychological approach to improving the outcomes of racially discordant medical interactions. Journal of General Internal Medicine, 28, 11431149. doi: 10.1007/s11606-013-2339-y

Peskowitz, M. (2005). The truth behind the mommy wars: Who decides what makes a good mother? Emeryville, CA: Seal House.

Pettigrew, T. F. (1997). Generalized intergroup contact effects on prejudice. Personality \& Social Psychology Bulletin, 23, 173-185.

Pettigrew, T. F. (1998). Intergroup contact theory. Annual Review of Psychology, 49, 65-85. 
Pettigrew, T. F., \& Tropp, L. R. (2006). A meta-analytic test of intergroup contact theory. Journal of Personality \& Social Psychology, 90, 751-783. doi: 10.1037/00223514.90.5.75

Pettigrew, T. F., Tropp, L. R., Wagner, U., \& Christ, O. (2011). Recent advances in intergroup contact theory. International Journal of Intercultural Relations, 35, 271-280. doi: 10.1016/j.ijintrel.2011.03.001

Postmes, T., \& Brunsting, S. (2002). Collective action in the age of the Internet: Mass communication and online mobilization. Social Science Computer Review, 20(3), 290301. doi: $10.1177 / 08939302020003006$

Powr, J. G., Murphy, S. T., \& Coover, G. (1996). Priming prejudice: How stereotypes and counter-stereotypes influence attribution of responsibility an credibility among ingrops and outgroups. Human Communication Research, 23, 36-58.

Prevention, C. f. D. C. a. (2011). Diagnoses of HIV infection in the United States and dependent areas. HIV Surveillance Report (Vol. 23). Atlanta, GA: Division of HIV/AIDS Prevention.

Reysen, S., Lloyd, J. D., Katzarska-Miller, I., Lemker, B. M., \& Foss, R. L. (2010). Intragroup status and social presence in online fan groups. Computers in Human Behavior, 26, 13141317. doi: $10.1016 /$ j.chb.2010.04.003

Riek, B. M., Mania, E. W., Gaertner, S. L., McDonald, S. A., \& Lamoreaux, M. J. (2010). Does a common ingroup identity reduce intergroup threat? Group Processes \& Intergroup Relations, 13, 403-423. doi: 10.1177/1368430209346701 
Rogers, S. J., \& White, L. K. (1998). Satisfaction with parenting: The role of marital happiness, family structure, and parents' gender. Journal of Marriage and the Family, 60, 293-308. doi: $10.2307 / 353849$

Rubin, S. E., \& Wooten, H. R. (2007). Highly educated stay-at-home mothers: A study of commitment and conflict. The Family Journal, 15(4), 336-345.

Ruble, R. A. (2011). Making ourselves understood: The role of previous experience, stereotypes, communication accommodation, and anxiety in Americans' perceptions of communication with chinese students. (3489964 Ph.D.), University of Kansas, Ann Arbor. ProQuest Dissertations \& Theses Full Text database.

Ruble, R. A., \& Zhang, Y. B. (2012, November). The role of previous experience, stereotypes, and anxiety in American's perceptions of communication with Chinese students. Paper presented at the annual meeting of the National Communication Association, Orlando, FL.

Ruble, R. A., \& Zhang, Y. B. (2013). Stereotypes of Chinese international students held by Americans. International Journal of Intercultural Relations, 37, 202-211. doi: 10.1016/j.ijintrel.2012.12.004

Rudman, L. A., \& Goodwin, S. A. (2004). Gender differences in automatic in-group bias: Why do women like women more than men like men? Journal of Personality and Social Psychology, 87, 494-509. doi: 10.1037/0022-3514.87.4.494

Sachdev, I., \& Bourhis, R. Y. (1985). Social categorization an power differentials in group relations. European Journal of Social Psychology, 15, 415-434.

Sachdev, I., \& Bourhis, R. Y. (1987). Status differentials and intergroup behaviour. European Journal of Social Psychology, 17, 277-293. 
Sachdev, I., \& Bourhis, R. Y. (2008). Multilingual communication and social identification. In J. Harwood \& H. Giles (Eds.), Intergroup communication: Multiple perspectives (Vol. 2, pp. 65-91). New York, NY: Peter Lang.

Saguy, T., Tausch, N., Dovidio, J. F., \& Pratto, F. (2009). The irony of harmony: Intergroup contact can produce false expectations for equality. Psychological Science, 20, 114-121. doi: $10.1111 / \mathrm{j} .1467-9280.2008 .02261 . x$

Schiappa, E., Gregg, P. B., \& Hewes, D. E. (2005). The parasocial contact hypothesis. Communication Monographs, 72, 92-115. doi: 10.1080/0363775052000342544

Secunda, V. (1995). Mothering instead of career--was it worth it? New Choices: Living Even Better After 50, 35, 50-53.

Sherif, M. (1966). Group conflict and cooperation. London, England: Routledge.

Shim, C., Zhang, Y. B., \& Harwood, J. (2012). Direct and mediated intercultural contact: Koreans' attitudes toward U.S. Americans. Journal of International \& Intercultural Communication, 5, 169-188. doi: 10.1080/17513057.2012.670715

Shpancer, N., Melick, K. M., Sayre, P. S., \& Spivey, A. T. (2006). Quality of care attributions to employed versus stay-at-home mothers. Early Child Development and Care, 176, 183193.

Sisters, B. (February 13, 2014). SAHM vs. WOHM. An inner war. Retrieved from: http://www.bitchinsisters.com/?s=inner+war\&submit=Search.

Smith, E. J. (1981). The working mother: A critique of the research. Journal of Vocational Behavior, 19, 191-211. doi: 10.1016/0001-8791(81)90058-0 
Smith, R. H. (2000). Assimilative and contrastive emotional reactions to upward and downward social comparisons. In J. Suls \& L. Wheeler (Eds.), Handbook of social comparison: Theory and research (pp. 173-200). Dordrecht, Netherlands: Kluwer Academic.

Soliz, J., Ribarsky, E., Harrigan, M. M., \& Tye-Williams, S. (2010). Perceptions of communication with gay and lesbian family members: Predictors of relational satisfaction and implications for outgroup attitudes. Communication Quarterly, 58, 77-95. doi: $10.1080 / 01463370903538622$

Soliz, J., \& Rittenour, C. E. (2012). Family as an intergroup arena. In H. Giles (Ed.), The Handbook of Intergroup Communication (pp. 331-342). New York, NY: Routledge.

Soliz, J., Thorson, A. R., \& Rittenour, C. E. (2009). Communicative correlates of satisfaction, family identity, and group salience in multiracial/ethnic families. Journal of Marriage and Family, 71, 819-832. doi: 10.1111/j.1741-3737.2009.00637.x

Steiner, L. M. (2007). Introduction: Our inner catfight. In L. M. Steiner (Ed.), Mommy wars: Stay-at-home and career moms face off on their choices, their lives, their families (pp. ixxxvi). New York, NY: Random House.

Stephan, W. G. (2014). Intergroup anxiety: Theory, research, and practice. Personality \& Social Psychology Review, 18, 239-255. doi: 10.1177/1088868314530518

Stephan, W. G., \& Stephan, C. W. (1985). Intergroup Anxiety. Journal of Social Issues, 41(3), $157-175$.

Stephan, W. G., \& Stephan, C. W. (1996). Predicting prejudice. International Journal of Intercultural Relations, 20, 409-426.

Stephan, W. G., Stephan, C. W., \& Gudykunst, W. B. (1999). Anxiety in intergroup relations: A comparison of anxiety/uncertainty management theory and integrated threat theory. 
International Journal of Intercultural Relations, 23, 613-628. doi: 10.1016/S01471767(99)00012-7

Suls, J., \& Wheeler, L. (2012). Social comparison theory. In P. A. M. Van Lange, A. W. Kruglanski, \& E. T. Higgins (Eds.), Handbook of Theories of Social Psychology: Volume One (pp. 460-481). Thousand Oaks, CA: SAGE.

Sun, F. (May 10, 2012). Behind the cover: Are you mom enough? Time. Retrieved from: http://lightbox.time.com/2012/05/10/parenting/\#1

Swann, W. B., Jr., Gomez, A., Seyle, D. C., Morales, J. F., \& Huici, C. (2009). Identity fusion: The interplay of personal and social identities in extreme group behavior. Journal of Personality and Social Psychology, 96, 995-1011.

Tabachnick, B. G., \& Fidell, L. S. (2013). Using multivariate statistics (6th ed.): Pearson. Tajfel, H. (1978). Differentiation between social groups: Studies in the social psychology of intergroup relations. London, England: Academic Press.

Tajfel, H., \& Turner, J. C. (1986). The social identity theory of intergroup behavior. In S. Worchel \& W. G. Austin (Eds.), Psychology of intergroup behavior (pp. 7-24). Chicago, IL: Nelson Hall.

Tannen, D. (1990). You just don't understand: Women and men in conversation. New York, NY: HarperCollins.

Tarkka, M.-T. (2003). Predictors of maternal competence by first-time mothers when the child is 8 months old. Journal of Advanced Nursing, 41, 233-240. doi: 10.1046/j.13652648.2003.02524.x

Taylor, P., Funk, C., \& Clark, A. (2007). From 1997 to 2007: Fewer mothers prefer full-time work. Washington, DC: Pew Research Center's Social \& Demographic Trends. 
Toalson, R. (March 15, 2015). Let's End the Mommy Wars, Once and For All. Huff Post Parents. Retrieved from: http://www.huffingtonpost.com/rachel-toalson/lets-end-themommy-wars-once-and-for-all_b_6834568.html

Turner, J. C., Hogg, M. A., Oakes, P. J., Reicher, S. D., \& Wetherell, M. S. (1987). Rediscovering the social group: A self-categorization theory. New York, NY: Blackwell.

Turner, R. N., Crisp, R. J., \& Lambert, E. (2007). Imagining intergroup contact can improve intergroup attitudes. Group Processes \& Intergroup Relations, 10, 427-441. doi: $10.1177 / 1368430207081533$

Turner, R. N., Hewstone, M., Voci, A., \& Vonofakou, C. (2008). A test of the extended intergroup contact hypothesis: The mediating role of intergroup anxiety, perceived inroup and outgroup norms, and inclusion of the outgroup in the self. Journal of Personality \& Social Psychology, 95, 843-860.

Umaña-Taylor, A. J., Yazedjian, A., \& Bámaca-Gómez, M. (2004). Developing the ethnic identity scale using Eriksonian and social identity perspectives. Identity: An International Journal of Theory and Research, 4, 9-38. doi: 10.1207/S1532706XID0401_2

Umaña-Taylor, A. J., Zeiders, K. H., \& Updegraff, K. A. (2013). Family ethnic socialization and ethnic identity: A family-driven, youth-driven, or reciprocal process? Journal of Family Psychology, 27, 137-146. doi: 10.1037/a0031105

Vallone, R. P., Ross, L., \& Lepper, M. R. (1985). The hostile media phenomenon: Biased perception and perceptions of media bias in coverage of the Beirut massacre. Journal of Personality and Social Psychology, 49, 577-585. doi: 10.1037/0022-3514.49.3.577 
Vang, M. H., \& Fox, J. (2014). Race in virtual environments: Competitive versus cooperative games with Black or White avatars. CyberPsychology, Behavior \& Social Networking, 17, 235-240. doi: 10.1089/cyber.2013.0289

Vezzali, L., Capozza, D., Stathi, S., \& Giovannini, D. (2012). Increasing outgroup trust, reducing infrahumanization, and enhancing future contact intentions via imagined intergroup contact. Journal of Experimental Social Psychology, 48, 437-440. doi:

10.1016/j.jesp.2011.09.008

Vincze, L., \& Harwood, J. (2012). TV language, cultivation, and perceived vitality of Hungarians in Slovakia. Communication Research Reports, 29, 266-273. doi: $10.1080 / 08824096.2012 .704600$

von Hippel, C., Issa, M., Ma, R., \& Stokes, A. (2011). Stereotype threat: Antecedents and consequences for working women. European Journal of Social Psychology, 41, 151-161. doi: 10.1002/ejsp.749

Vonk, R., \& Olde-Monnikhof, M. (1998). Gender subgroups: Intergroup bias within the sexes. European Journal of Social Psychology, 28, 37-47.

Wade, M. L., \& Brewer, M. B. (2006). The structure of female subgroups: An exploration of ambivalent stereotypes. Sex Roles, 54, 753-765. doi: 10.1007/s11199-006-9043-x

Walther, J. B. (1996). Computer-mediated communication: Impersonal, interpersonal, and hyperpersonal interaction. Communication Research, 23(1), 3-43.

Walther, J. B. (2009). Computer-mediated communication and virtual groups: Applications to interethnic conflict. Journal of Applied Communication Research, 37, 225-238. doi: $10.1080 / 00909880903025937$ 
Wang, Z., Walther, J. B., \& Hancock, J. T. (2009). Social identification and interpersonal communication in computer-mediated communication: What you do versus who you are in virtual groups. Human Communication Research, 35, 59-85. doi: 10.1111/j.14682958.2008.01338.x

West, T. V., Pearson, A. R., Dovidio, J. F., Shelton, J. N., \& Trail, T. E. (2009). Superordinate identity and intergroup roommate friendship development. Journal of Experimental Social Psychology, 45, 1266-1272.

Wilder, D. A. (1993). The role of anxiety in facilitating stereotypic judgments of outgroup behavior. In D. M. Mackie \& D. L. Hamilton (Eds.), Affect, cognition, and stereotyping: Interactive processes in group perception (pp. 87-109). San Diego, CA: Academic Press.

Willer, E. K., \& Cupach, W. R. (2011). The meaning of girls' social aggression: Nasty or mastery? In W. R. Cupach \& B. H. Spitzberg (Eds.), The dark side of close relationships II (pp. 297-326). New York, NY: Routledge.

Willer, E. K., \& Soliz, J. (2010). Face needs, intragroup status, and women's reactions to socially aggressive face threats. Personal Relationships, 17, 557-571. doi: 10.1111/j.14756811.2010.01297.x

Wood, J. V. (1989). Theory and research concerning social comparisons of personal attributes. Psychological Bulletin, 106, 231-248. doi: 10.1037/0033-2909.106.2.231

Wright, S. C., Aron, A., McLaughlin-Volpe, T., \& Ropp, S. A. (1997). The extended contact effect: Knowledge of cross-group friendships and prejudice. Journal of Personality and Social Psychology, 73, 73-90. doi: 10.1037/0022-3514.73.1.73 
Zimmerman, T. S., Aberle, J. T., Krafchick, J. L., \& Harvey, A. M. (2008). Deconstructing the 'mommy wars': The battle over the best mom. Journal of Feminist Family Therapy: An International Forum, 20, 203-219. doi: 10.1080/08952830802264524 


\section{Appendix A}

\section{Procedures Flow Chart}

Kelly recruited mothers with children under 6 years old via online announcements

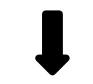

Qualified and interested mothers emailed Kelly

$\downarrow$

Kelly used random order distribution system to assign participant mothers to one of 13 stereotype conditions (Study 1) or 4 CIIM representational mediator conditions (Study 2)

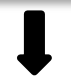

Kelly distributed response emails to participant mothers that include instructions and a link to an online questionnaire (based on random assignments)

Participant mothers clicked link and completed instruments on the first part of the online questionnaires (i.e., demographic questions, ingroup identification, intergroup contact experiences)

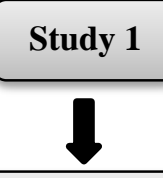

Participant viewed one of 13 target SAHM/WM stereotype conditions

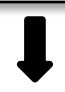

Participant mothers completed instruments on the second part of the online questionnaire (i.e., outgroup

attitudes, affective and behavioral responses, intergroup anxiety, and willingness to communicate)

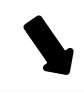

Participant mothers were asked to advance to the online interaction or exit the study

Participant mothers were debriefed and directed to the Comma Mommas Facebook group and willingness to communicate)
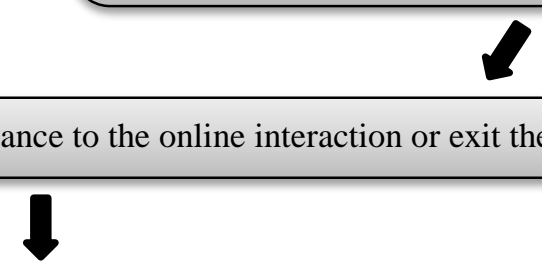

Participant mothers were randomly assigned to read 1 of 4 target outgroup mother messages written in accordance to the four CIIM representational mediator conditions

Participant mothers completed instruments on the second part questionnaire (i.e., reliance on stereotypes, outgroup attitudes, perceptions of outgroup typicality, helping, 
Appendix B

\section{Study One Stereotype Condition Descriptions}

\section{SAHM Stereotype Condition \#1: Busy \& Overworked}

As a stay-at-home mother with two children, Michelle is extremely busy and overworked. She feels overburdened and overwhelmed by her never-ending workload. She overcommits herself and then feels torn between her many responsibilities. As a result, she often feels stressed because there is never enough time in the day to accomplish all that she wants to accomplish. When she finally gets a chance to sit down at the end of each busy day, she is exhausted and worn out.

\section{SAHM Stereotype Condition \#2: Executive of the Home}

As a stay-at-home mother with two children, Michelle considers herself the executive of her home. In this sense, her life is centered on her family's needs and household responsibilities. She loves and cares for her family and also manages everyone's schedules, activities, and finances. She is very loving and involved in her children's lives. When she is not spending quality time with her children, she is maintaining her household, like a CEO of the home would do. She also enjoys (and is very good at) cooking, baking, and decorating her home.

\section{SAHM Stereotype Condition \#3: Lazy, Selfish, \& Lost}

As a stay-at-home mother with two children, Michelle is lazy. She is not attentive. She is not nurturing. She is not hardworking. She does not want to hold a job or have hobbies outside the home. Although she is disinterested and unmotivated about most things, she is selfish and will 
still put her individual needs ahead of her children's needs. In fact, she does not care to build strong relationships with her children and does not prioritize her family.

\section{SAHM Stereotype Condition \#4: Ideal Mother}

As a stay-at-home mother with two children, Michelle is the ideal mother. She is very maternal and has good instincts about parenting and child development. She is always putting others' needs ahead of her own and giving her family pure, unconditional love. Because of this, she has close relationships with her children, who respect and admire her. Plus, she is always on top of her to-do list, accomplishing everything she needs to get done in a day-quickly and with very few mistakes! She is a role model and supermom all in one! She is truly amazing!

\section{SAHM Stereotype Condition \#5: Balancing Work, Family, \& Life}

As a stay-at-home mother with two children, Michelle is constantly balancing her work, family, and life responsibilities. She wears many hats and is constantly involved in this balancing act. On any given day, she is simultaneously focusing on her work duties, raising her children, and maintaining her household. She has learned to juggle many work-related tasks and effectively balance her work, family, and life responsibilities.

\section{SAHM Stereotype Condition \#6: Non-traditional}

As a stay-at-home mother with two children, Michelle is a non-traditional woman. She equally divides decision-making, household chores, and parenting responsibilities with her partner. She fully supports women's rights movements and feels empowered to make choices for the good of herself and her family. On occasion, she has assumed the role of family provider. As a feminist, 
she considers herself modern, liberal, and progressive.

\section{SAHM Stereotype Condition \#7: Traditional}

As a stay-at-home mother with two children, Michelle is a traditional woman. She enjoys fulfilling the two feminine roles that are expected of women: wife and mother. She always wanted to get married, have children, and stay home to care for her family. She believes her main purposes in life are to raise her children, maintain her household, and satisfy her husband's needs. Her husband is responsible for working and earning money for the family. Against feminism, she is strictly traditional in her roles and thinking.

\section{WM Stereotype Condition \#1: Overworked with no free time}

As a working mother with two children, Michelle is overextended and has no free time. She typically feels overburdened and overwhelmed by her workload. She overcommits herself, sacrifices all of her free time, and then feels torn between her many responsibilities. As a result, she feels stressed because there is never enough time in the day to accomplish all that she wants. When she finally gets a chance to sit down at the end of each day, she is exhausted and worn out.

\section{WM Stereotype Condition \#2: Hardworking \& Determined}

As a working mother with two children, Michelle is determined and hardworking. Despite her busy schedule, she is motivated to accomplish all of her responsibilities each day. Her dedication to her family and job, coupled with her ambitious personality, helps her effectively multi-task. She knows she must work hard and stay focused if she wanted to accomplish all of her goals. 


\section{WM Stereotype Condition \#3: Supermom}

As a working mother with two children, Michelle is a supermom. She really has it all! She is always on top of her to-do list, accomplishing everything she needs to get done in a dayquickly and with very few mistakes! She is also very maternal and has good instincts about parenting and child development. Because of this, she has close relationships with her children, who respect and admire her. She is a role model and supermom all in one! She is truly amazing!

\section{WM Stereotype Condition \#4: Flexible \& Family-oriented}

As a working mother with two children, Michelle is family-oriented and flexible. Despite her work schedule, her patient, easy-going, and adaptable personality allows her to prioritize her family's needs and stay involved in her children's lives. She does not mind changing her plans on short notice to accommodate and spend time with her family.

\section{WM Stereotype Condition \#5: Non-traditional}

As a working mother with two children, Michelle is a non-traditional woman. She is the breadwinner, earning the majority of the money for her family. She equally divides decisionmaking, household chores, and parenting responsibilities with her partner. She fully supports women's rights movements and feels empowered to make choices for the good of herself, her career, and her family. As a feminist, she is modern, liberal, and progressive.

\section{WM Stereotype Condition \#6: Traditional}

As a working mother with two children, Michelle is a traditional woman. She enjoys fulfilling the two feminine roles that are expected of women: wife and mother. She believes her main 
purposes in life are to raise her children, maintain her household, and satisfy her husband's needs. Her husband is responsible for working and earning the majority of the money for her family, which allows her to enjoy many of life's financial luxuries. She feels very lucky to be able to spend a lot of time with her children and likes to know everything about her children's decisions, activities, and personal relationships. Although she is often overbearing, she believes she must hover over her children in order to protect them from difficult situations and solve their problems. 
Appendix C

Study One Pilot Study Questionnaire

Instructions: First, read the following description VERY carefully. Then, answer the questions below and on the back based on what you read.

\section{DESCRIPTION:}

As a stay-at-home mother with two children, Michelle is lazy. She is not attentive. She is not nurturing. She is not hardworking. She does not want to hold a job or have hobbies outside the home. Although she is disinterested and unmotivated about most things, she is selfish and will still put her individual needs ahead of her children's needs. In fact, she does not care to build strong relationships with her children and does not prioritize her family.

Based on what you read in the previous description, provide 1-4 adjectives to describe Michelle. These adjectives can be single words or multi-word phrases. You can use words and phrases from the previous description or create your own. Write these adjectives on the lines below.

Please continue onto the back for additional questions. 
Based on what you read in the description on the previous page, choose the one description (from the options below) that you think best represents Michelle. Please do not offer your personal opinion of Michelle; instead how you think she was described. You may refer back to the description before responding.

A mother who balances work, family, and life

A lazy and selfish mother

A family-oriented, executive of the home

A non-traditional woman

An ideal mother

A busy and overworked mother

A traditional woman

Instructions: Please complete the following demographic questions.

1. Your sex (please circle): Male Female 2. Your age:

3. Please indicate (by circling) the race/ethnicity with which you identify?
a. White
b. Black
c. Asian or Asian American
d. Hispanic or Latino/a
e. Native American
f. Pacific Islander
g. Middle Eastern
h. Bi/Multi-racial or Other? Please specify:

4. Do you have any children (please circle)? Yes No

a. If you have children:

i. Please indicate how many:

ii. Please indicate their ages:

iii. Please indicate if you consider yourself a (please circle):

1. Stay-at-home mother

2. Working mother

3. Father

4. Other:

5. Was your mother a (please circle):
a. Stay-at-home mother
b. Working mother
c. Other: 


\section{Appendix D}

\section{Online Recruitment Announcement}

Hello mothers of [insert name of online recruitment source]! My name is Kelly Odenweller. I am a Ph.D. Candidate/Visiting Instructor in the Department of Communication Studies at West Virginia University (WVU)—and a mother of two young children!

I am currently working on my doctoral dissertation (WVU IRB Protocol \#1412508170), under the direction of Dr. Christine E. Rittenour, an Assistant Professor in the Department of Communication Studies at WVU. My dissertation is a continuation of previous research I have conducted to explore mothers' communication. One (unexpected) thing I have learned in my research so far is that mothers long for more meaningful adult relationships, including friendships with other mothers. Thus, my dissertation is focused on initiating mother-to-mother relationships and demonstrating the benefits of these relationships on mothers' wellbeing.

I am looking for mothers who are willing to participate in my dissertation. Participation, which should take no longer than 20 minutes, will involve: (1) completing a brief online questionnaire and (2) chatting with me and one other mother via a web/text-based chat technology (e.g., Google, Facebook, Windows Live, Yahoo). These interactions will be brief, causal, and non-invasive. All participating mothers will be entered into a drawing for a chance to win one of four $\mathbf{\$ 5 0}$ retailer gift cards.

If you are interested in participating, you are a mother, and you have at least one child who is 6 years old or younger, please email me at kodenwel@mix.wvu.edu by next Friday, February 6, 2015 at 11:59 p.m. so we can get the process started. Thank you in advance for your help! I GREATLY appreciate you! 


\section{Appendix E}

\section{First Response Email to Participant Mothers}

\section{Hello Mommas:}

Thank you so much for your interest in my IRB-approved (Protocol \#1412508170)

dissertation focused on mothers' adult relationships. In order to efficiently schedule mothers for the online interactions, I am waiting to distribute information about the study until I receive all participant requests on February 6, 2015 at 11:59 p.m. Shortly after this deadline, you will receive another email from me with specific instructions about participating. Once you receive this email, you will have approximately 3 days to complete your participation to be eligible for the $\mathbf{\$ 5 0}$ gift card. Again, thank you for your interest in this study and your patience while I wait for other mothers to contact me. Have a great weekend!

Sincerely,

Kelly

Christine E. Rittenour, Ph.D.

Principal Investigator

Assistant Professor

Department of Communication Studies

West Virginia University

(304) 293-3905

christine.rittenour@mail.wvu.edu

Kelly G. Odenweller, M.A.

Co-Investigator

Ph.D. Candidate/Visiting Instructor

Department of Communication Studies

West Virginia University

(614) 296-5569

kodenwe1@mix.wvu.edu 
Appendix F

\section{Second Response Email to Participant Mothers}

\section{Hello Mommas:}

I'm very excited to announce the official "launch" of my dissertation focused on mothers' relationships, communication, and wellbeing. Thank you for your continued interest in this study and your patience as I gathered confirmations from other mothers. (And many, many thanks to those of you who helped me recruit additional mothers over the last two weeks!). To obtain specific instructions about participating, provide your consent, and begin the study, please click this link: http://wvu.qualtrics.com/SE/?SID=SV_0kt4gbUabeIOy2h. To be eligible for the $\$ 50$ gift cards, you must complete your participation before February 16, 2015 at 5 p.m. If you have any questions throughout the process, please don't hesitate to contact me. Thank you so much for you valuable time and input!

Kelly

Note: This dissertation is being conducted under the direction of Dr. Christine Rittenour, an Assistant Professor in the Department of Communication Studies at West Virginia University. West Virginia University's Institutional Review Board has acknowledgement of this study one file (Protocol \#1412508170).

\section{Christine E. Rittenour, Ph.D. \\ Principal Investigator Assistant Professor Department of Communication Studies West Virginia University}


(304) 293-3905

christine.rittenour@mail.wvu.edu

Kelly G. Odenweller, M.A.

Co-Investigator

$\mathrm{Ph}$.D. Candidate/Visiting Instructor

Department of Communication Studies

West Virginia University

(614) 296-5569

kodenwel@mix.wvu.edu 
Appendix G

Study One Online Questionnaire 


\section{Dear Participant:}

You are being asked to participate in an IRB-approved research study (Protocol \#1412508170). This research study is being conducted by Dr. Christine E. Rittenour, an Assistant Professor in the Department of Communication Studies at West Virginia University and the principal investigator, and Kelly G. Odenweller, a Ph.D. Candidate/Visiting Instructor in the Department of Communication Studies at West Virginia University and the co-investigator. This research is focused on mothers' adult friendships, communication, and wellbeing. This study will fulfill requirements toward earning a Ph.D. in Communication Studies for the co-investigator.

To qualify for participation, you must be: (a) female, (b) at least 18 years old, (c) have at least one child who is 6 years old or younger. Participation in this study will take approximately $\mathbf{2 0}$ minutes and will proceed in the following two steps:

1. The first step is completing a 10-minute online questionnaire. This questionnaire will ask you to provide basic information about yourself, learn more about the mother with whom you will eventually be interacting, and schedule your online chat session for a day/time that is most convenient for you between January 26 , 2014 and February 13, 2014.

2. The second step is participating in the 10-minute online interaction. On your scheduled date/time, I will join you and the other mother in a Facebook Chat session. I do not anticipate these interactions taking longer than 10 minutes. However, should you and your partner "hit it off" and want to continue your conversation without me, you will be able to exchange personal contact at the end of the study.

Your participation in this study is completely voluntary. You may skip questions on the questionnaire, refrain from interacting in the online chat session, or withdraw your consent at any time. These decisions will not harm your relationship with the researchers or the department/university with which the researchers are affiliated, result in any loss of benefits for which you are otherwise entitled, or affect your relationship with any other mother in the study. Any information about you that is obtained as a result of your participation in this research will be kept as confidential as legally possible. In any conference presentations or publications that result from this research, neither your name nor any information from which you might be identified will be published without your additional consent.

If you do wish to discontinue your participation in the study, please let me know and I can easily and confidentially swap in another mother without your partner knowing you have withdrew. It is MORE important that I receive your honest and candid feedback (whether it's positive or negative) during this process-so please do not feel obligated to withhold your opinions or engage in something with which you are not completely comfortable. No one (including the researchers) will ever be able to connect you to your specific responses as we will keep all of your personal information completely private (even during the actual online chat session). Only should you choose to continue a relationship with the other mother could she have your personal information-but this will be at your discretion. In the event new information becomes available that may affect your willingness to participate in this study, this information will be given to you so that you can make an informed decision about whether or not to continue your participation.

There are no fees required to participate in this study. In exchange for you valuable time and input, all participating mothers will be entered into a drawing for a chance to win one of four $\$ 50$ retailer gift cards. If you withdraw before the end of the study, you will still be eligible for the gift card.

There are no known or expected risks from participating in this study, except for mild frustration or discomfort related to answering questions on the online questionnaire or participating in the online interaction. Should it become necessary, free assistance is available to you through the WVU Carruth Center for Psychological and Psychiatric Services (304-293-4431) or the National Alliance on Mental IIIness (1-800-950-6264).

Because you will have the opportunity to connect with another mother during the online interaction, the potential exists for you to make a new friend. However, similar to your current friendships, you are free to put as much or as little effort into this relationship as you desire and feel comfortable with following the study. Even if you do not receive any direct benefits from this study, the knowledge gained from this study provide insight into mothers' communication and relationships. This information will be invaluable in helping other mothers. 
If you have any questions, concerns, or complaints about this research study, you should contact Dr. Christine E. Rittenour or Kelly G. Odenweller at the emails or phone numbers listed below. If you have questions related to your rights as a research participant, please contact WVU's Office of Research Integrity \& Compliance at (304) 293-7073. Please note that West Virginia University's Institutional Review Board has approval of this study on file (Protocol \#1412508170). If you have read the terms and the conditions of the study and willingly agree to participate in this study, please click the box below to advance to the online questionnaire.

Christine E. Rittenour, Ph.D.

Principal Investigator

Assistant Professor

Department of Communication Studies

West Virginia University

(304) 293-3905

christine.rittenour@mail.wvu.edu

Kelly G. Odenweller, M.A.

Co-Investigator

Ph.D. Candidate/Visiting Instructor

Department of Communication Studies

West Virginia University

(614) 296-5569

kodenwel@mix.wvu.edu

I have read the terms and conditions of this research study and willingly agree to participate.

Your participation in this study begins with the following questionnaire. Please respond to the questions on the following pages as honestly as possible.

Instructions: Please answer the following demographic information about yourself and your children.

Your sex:

Male

Female

Your age:

The number of children you have: 
Your child's/children's age(s):

Note: If you have more than one child, please report all of your children's ages by holding down the control button to select more than one option in the drop down menu below.

\begin{tabular}{|l|}
\hline under 1 month \\
1 month \\
2 months \\
3 months \\
4 months \\
5 months \\
6 months \\
7 months \\
8 months \\
9 months
\end{tabular}

The ethnicity with which you identify:

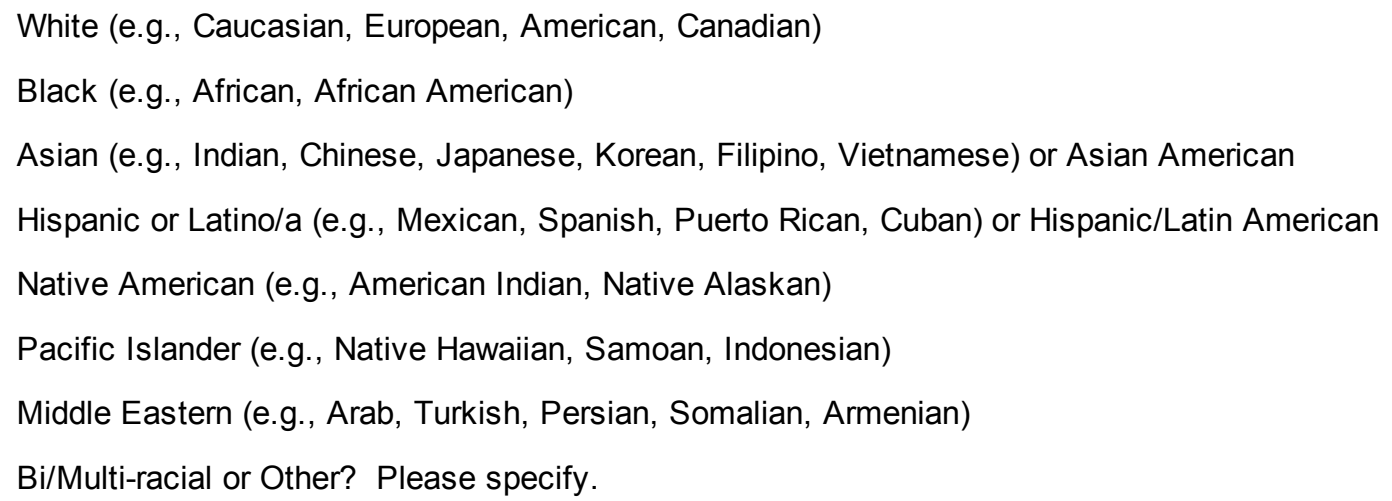

Your relationship status:

Single

Engaged or in a long-term, committed relationship

Married/Remarried

Divorced

Widowed

\section{Your sexual orientation:}

Heterosexual

Gay or Lesbian

Bi-sexual 
Highest level of education you have completed:

Some high school

High school diploma/GED

Professional certificate/license

Some college credits

Bachelor's degree

Graduate degree (e.g., Master's, Ph.D., J.D., MBA)

Your annual household income:

Instructions: Please answer the following questions about your identity as a mother.

We are aware that the following labels are often unable to capture the full depth of mothers' work/family arrangements and identities. However, for the purposes of this study, we ask that you select the one that best describes you.

working mother

stay-at-home mother

If you do not identify as working or stay-at-home mother (and felt forced to choose in the previous question), please explain why (in as much detail as possible) these labels are not ideal for you. What term or phrase would best describe your family/work arrangements? 
The image below depicts five potential relationships between you as an individual and stay-at-home/working mothers as a group. Which of the options in the image best represents how SIMILAR you feel to your stay-athome/working mother group? Chose the letter that corresponds to that option, with "A" being the appropriate option if you feel completely different and separate from your stay-at-home/working mother group and " $F$ " being the appropriate option if you feel completely identical to your stay-at-home/working mother group.
A
B
C
D
$\mathrm{E}$
F

Group


Instructions: Using the scale below, indicate your how well each statement describes you.

Does not describe me AT

ALL
UNDECIDED

as to whether

it describes

me well or

not
Describes me VERY WELL

I have read books, magazine, newspapers, or other materials that have taught me about my group of stay-at-home/working mothers.

I have learned about my group of

stay-at-home/working mothers by doing things such as reading (books, magazines, newspapers), searching the Internet, or keeping up with current events.

I have participated in activities that have exposed me to my group of stay-at-home/working mothers.

I am clear about what being a

stay-at-home/working mother means to me.

I have participated in activities that have taught me about my group of stay-at-home/working mothers.

If I could choose, I would prefer to be of a different mom group (e.g., stay-at-home/working mothers).

I dislike my group of stay-at-home/working mothers.

I have not participated in any activities that would teach me about my group of stay-at-home/ working mothers.

I am not happy with my group of stay-at-home/working mothers.

I have experienced things that reflect my group of stay-at-home/working mothers, such as reading books, watching movies, or attending events.

I know what being a stay-at-home/working mothers means to me.

I have a clear sense of what being a stay-at-home/working mother means to me.

I feel negatively about my group of stay-at-home/working mothers.

I wish I were of a different mom group stay-at-home/working mothers.

I have attended events that have helped me learn more about my group of stay-at-home/working mothers.

I understand how I feel about my group of stay-at-home/working mothers.

My feelings about my group of

stay-at-home/working mothers are mostly negative.

The questions on the next four pages will ask you about your experiences interacting with stay-at-home mothers, working mothers, and non-mothers (i.e., men and women who are NOT parents). 
Instructions: Using the scale below, indicate your level of agreement with each statement in relation.

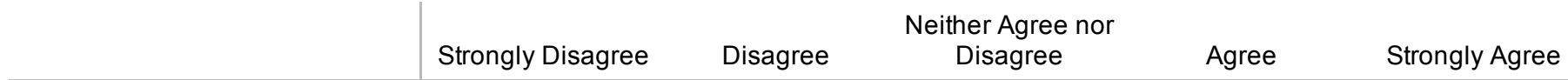

All of my friends and I have similar work/family arrangements.

I have close friends who are stay-at-home mothers.

I speak to stay-at-home mothers on a regular basis.

I have at least one

acquaintance who is a stay-athome mother.

I have never talked to a stay-athome mother.

I have close friends who are working mothers.

I speak to working mothers on a regular basis.

I have at least one acquaintance who is a working mother.

I have never talked to a working mother.

I have close friends who are not mothers.

I speak to people who are not mothers on a regular basis.

I have at least one acquaintance who is not a mother.

I have never talked to someone who is not a mother.

Instructions: The questions and scales on this page address your past interactions with STAY-AT-HOME mothers. As a note, the same question will be repeated five times, but the scale below the question changes each time to assess different aspects of these interactions. Please respond to each scale individually.

When you came in contact with stay-at-home mothers in the past, overall you perceived your interactions to be:

Imbalanced in

terms of

power/status

Balanced in terms of power/status

When you came in contact with stay-at-home mothers in the past, overall you perceived your interactions to be: 
When you came in contact with stay-at-home mothers in the past, overall you perceived your interactions to be:

Superficial

Intimate

When you came in contact with stay-at-home mothers in the past, overall you perceived your interactions to be:

When you came in contact with stay-at-home mothers in the past, overall you perceived your interactions to be:

Uncomfortable

Pleasant

Instructions: The questions and scales on this page address your past interactions with WORKING mothers. As a note, the same question is repeated five times, but the scale below the question changes each time to assess different aspects of these interactions. Please respond to each scale individually.

When you came in contact with working mothers in the past, overall you perceived your interactions to be:

$$
\begin{gathered}
\text { Imbalanced in } \\
\text { terms of } \\
\text { power/status }
\end{gathered}
$$

When you came in contact with working mothers in the past, overall you perceived your interactions to be:

$$
\text { Involuntary }
$$

$$
\text { Voluntary }
$$

When you came in contact with working mothers in the past, overall you perceived your interactions to be:

$$
\text { Superficial }
$$

When you came in contact with working mothers in the past, overall you perceived your interactions to be: 
When you came in contact with working mothers in the past, overall you perceived your interactions to be:

Uncomfortable

Instructions: The questions and scales on this page address your past interactions with NON-MOTHERS. As a note, the same question is repeated five times, but the scale below the question changes each time to assess different aspects of these interactions. Please respond to each scale individually.

When you came in contact with non-mothers in the past, overall you perceived your interactions to be:

$$
\begin{gathered}
\text { Imbalanced in } \\
\text { terms of } \\
\text { power/status }
\end{gathered}
$$

When you came in contact with non-mothers in the past, overall you perceived your interactions to be:

$$
\text { Involuntary }
$$

When you came in contact with non-mothers in the past, overall you perceived your interactions to be:

$$
\text { Superficial }
$$

When you came in contact with non-mothers in the past, overall you perceived your interactions to be:

Competitive

Cooperative

When you came in contact with non-mothers in the past, overall you perceived your interactions to be: 
I have less time to maintain adult friendships than I did before I had children.

\begin{tabular}{|c|c|c|c|}
\hline trongly Disagree & Disagree & $\begin{array}{l}\text { Somewhat } \\
\text { Disagree }\end{array}$ & $\begin{array}{c}\text { Neither Agree nor } \\
\text { Disagree }\end{array}$ \\
\hline
\end{tabular}

I have less interest in maintaining adult friendships than I did before I had children.

\begin{tabular}{|c|c|c|c|c|}
\hline Strongly Disagre & Disagree & $\begin{array}{c}\text { Somewhat } \\
\text { Disaqree }\end{array}$ & $\begin{array}{c}\text { Neither Agree nor } \\
\text { Disagree }\end{array}$ & Somewhat Agree \\
\hline
\end{tabular}

It is easier to maintain adult friendship through online channels (e.g., email, Facebook, instant messenger) compared to face-to-face or over the phone.

\begin{tabular}{|c|c|c|c|c|c|c|}
\hline Strongly Disagree & Disagree & $\begin{array}{l}\text { Somewhat } \\
\text { Disagree }\end{array}$ & $\begin{array}{c}\text { Neither Agree nor } \\
\text { Disagree }\end{array}$ & Somewhat Agree & Agree & Strongly Agree \\
\hline
\end{tabular}

I wish I could maintain more adult friendships than I currently do.

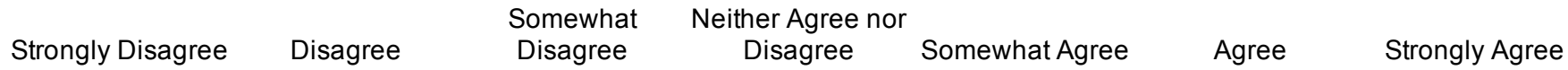

Before we randomly pair you with another mother involved in this project, please provide a brief description of yourself that we can use to introduce you to this mother. She has been asked to do this as well, and once you complete this section for yourself, you will have the opportunity to learn more about your partner on the next page. There are no right or wrong responses. Feel free to share any details about yourself, your children, your partner/spouse, your mother-in-law, etc. that you would like us to pass along to the other mother. This is also a great place to discuss what you like to do with your free time, your favorite hobbies/leisure activities, what you do to unwind and relax, what you would love to do if you had a babysitter tonight, etc. 
On the next page, you will see a description of the mother who has been randomly selected to join us in the 10-minute online chat session following this questionnaire. Please read this description carefully before advancing to the following page.

As a stay-at-home mother with two children, Michelle is extremely busy and overworked. She feels overburdened and overwhelmed by her never-ending workload. She overcommits herself and then feels torn between her many responsibilities. As a result, she often feels stressed because there is never enough time in the day to accomplish all that she wants to accomplish. When she finally gets a chance to sit down at the end of each busy day, she is exhausted and worn out.

Instructions: Please answer the following questions about the mother you read about on the previous page (and will eventually interact with in the online chat session).

Did you carefully read the description of the other mother?

yes

no

This mother is a:

working mother

stay-at-home mother

Based on the description you read about the other mother, which of the following options best describe her?

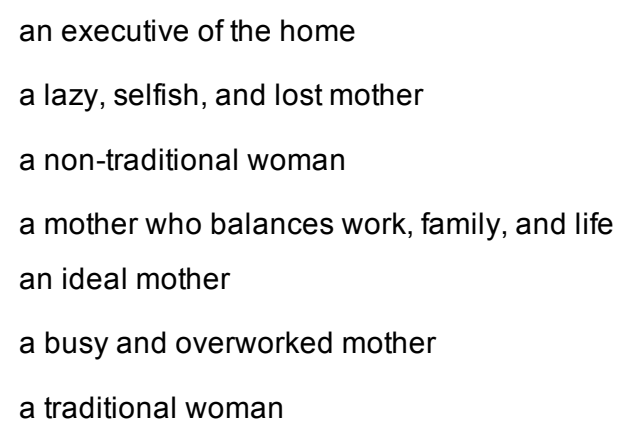


Instructions: Using the following thermometer as a scale, indicate how you feel about the mother with which you have been randomly paired. While completing this instrument, associate warm and favorable attitudes with higher temperatures on the thermometers and associate cold and unfavorable attitudes with lower temperatures on the thermometers. Choose the "temperature readings" that best represent your attitudes toward the other mother.

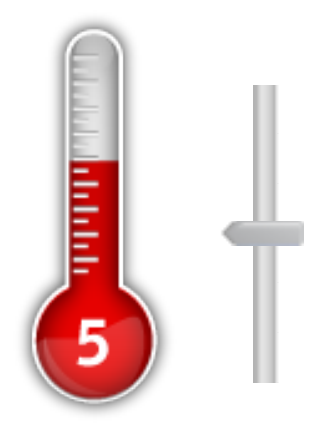

Instructions: Using the scale below, indicate the degree to which you feel each emotion when thinking about the other mother.

Admiration

Anger

Envy

Happy

Accepted

Contempt

Confident

Impatient

Uncertain

Pride

Fear

Careful

Pity

Awkward

Disgust

Sympathy

Jealousy

Defensive 
Suspicious

Self-conscious

Irritated

Instructions: Using the scale below, indicate the degree to which you would be inclined to perform each behavior listed in relation to the other mother.

Fight her
Attack her
Protect her
Exclude her
Have nothing to
Cooperate with
Keep her at a dist
Associate with
Avoid her
Argue with her
Demean her
Confront her
Oppose her
Help her

Never

Always

Protect her

Exclude her

to do with her

Associate with her

Help her

$\begin{array}{ll}0 & 0 \\ 0 & 0 \\ 0 & 0 \\ 0 & 0 \\ 0 & 0 \\ 0 & 0 \\ 0 & 0 \\ 0 & 0 \\ 0 & 0 \\ 0 & 0 \\ 0 & 0 \\ 0 & 0 \\ 0 & 0 \\ 0 & 0\end{array}$

Instructions: Using the scale below, indicate your level of agreement with each statement.

I would like to participate in a program to help build connections between stay-at-home and working mothers.

$\begin{array}{lcccc} & \text { Somewhat } & \text { Neither Agree nor } \\ \text { Strongly Disagree } & \text { Disagree } & \text { Disagree } & \text { Disagree } & \text { Somewhat Agree Agree Strongly Agree }\end{array}$

I would be willing to speak to the other mother in an online chat session.

\begin{tabular}{|c|c|c|c|c|c|c|}
\hline Strongly Disagree & Disagree & $\begin{array}{l}\text { Somewhat } \\
\text { Disagree }\end{array}$ & $\begin{array}{c}\text { Neither Agree nor } \\
\text { Disagree }\end{array}$ & Somewhat Agree & Agree & Strongly Agree \\
\hline
\end{tabular}


I would be willing to connect with the other mother on social media (e.g., Facebook, Twitter).

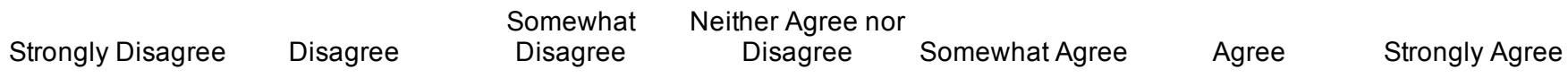

I would be willing to meet the other mother face-to-face.

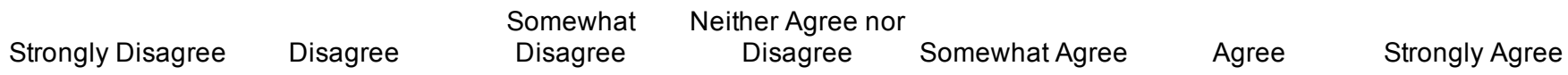

Thank you for completing the previous questions. Please advance to the next page to schedule your online chat session with the other mother. If-for whatever reason-you do not wish to interact with the other mother, please click "exit study now" below.

Schedule online chat session now

Exit study now

Please choose the option below that best explains why you chose to exit the survey now (i.e., before participating in the online interaction). Please note the other mother will never see this response. Additionally, your response will not jeopardize your entrance into the raffle for the $\$ 50$ gift card. I simply want to know what you are thinkingplease be honest and candid.

I no longer have time to participate in the 10-minute online interaction

I do not feel comfortable talking in online chat sessions

I do not want to interact with the specific mother you have paired me with

I am worried the other mother will not like me

Other (please specify):

Thank you so much for your time and input! You have now reached the end of the study. So that you could best help with my goal of connecting mothers, I withheld some information from you at the beginning of the study. I would like to share that information with you now.

Ultimately, my research is devoted to minimizing what the media has labeled the "mommy wars," intense rivalries among mothers based on a variety of parenting decisions and family lifestyles. You may have watched a news program or read an article about, or even personally experienced, these "wars" as a mother. My dissertation (WVU IRB Protocol \#1412508170) is focused specifically on stay-at-home and working mothers, and I am using the data you have just provided to help me understand the attitudes, feelings, and communication between these women.

Initially, I said you would be participating in an online interaction with another mother, about whom you read a brief description. This mother was not a real person but a description based on previous research on stereotypes of stayat-home and working mothers. The questions you answered about this mother comprised the entire data collection. Again, this data will be used to inform future efforts to move from anxious or avoidant communication (i.e., "mommy wars") to more inclusive and supportive communication among mothers. Now that you have provided this 
confidential data, you may engage in what you set out to do, and what was advertised in my initial message: connect with other mothers.

I have created a private Facebook group where you can join all of the mothers who generously gave their time for this study and begin interacting with them as you likely hoped to do when you signed on for this study. There is NO obligation to join this group and this Facebook page is NOT part of the study (i.e., nothing you post on this page may be used for research purposes). Aside from posting my own/participating in your conversations about motherhood, family, work, and life, I will use this group to make announcements about the winners of the $\$ 50$ gift cards, results of my recent research studies, and future research opportunities. If you are interested, please follow the link here: Comma Mommas.

As a reminder, the anonymous responses you provided will never be connected to you as not even I know who shared which responses. However, if you would like your data to be withdrawn from the study, please let me know. Additionally, if required, free assistance is available to you through the WVU Carruth Center for Psychological and Psychiatric Services (304-293-4431) or the National Alliance on Mental IIIness (1-800-950-6264).

As I will be collecting data for my dissertation until February 13, 2014 and would like to preserve the integrity of my study design, I would greatly appreciate it if you did not share this information with other mothers who might become future participants. I will announce the closing of the study via the Facebook group mentioned above. At that time, you will be completely free to discuss your participation publicly. If you would like more information regarding this research project now, please contact me privately at kodenwel@mix.wvu.edu or 614-296-5569. Thank you again for your involvement in my dissertation. I wish you and yours a fabulous 2015!

Sincerely,

Kelly

Christine E. Rittenour, Ph.D.

Principal Investigator

Assistant Professor

Department of Communication Studies

West Virginia University

(304) 293-3905

christine.rittenour@mail.wvu.edu

Kelly G. Odenweller, M.A.

Co-Investigator

Ph.D. Candidate/Visiting Instructor

Department of Communication Studies

West Virginia University

(614) 296-5569

kodenwel@mix.wvu.edu 


\section{Appendix $\mathrm{H}$}

\section{Comma Mommas Facebook Group}

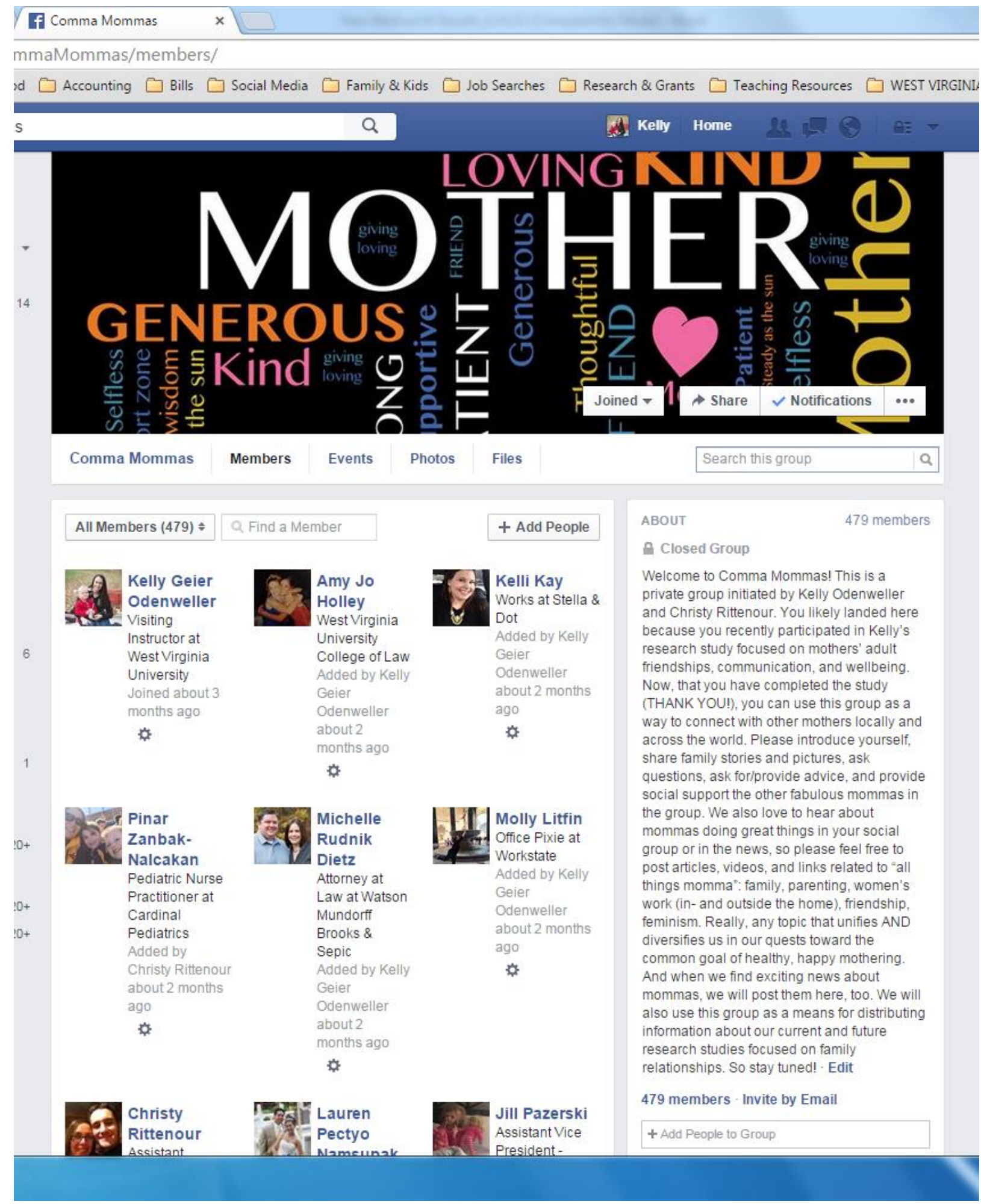


Appendix I

\section{Feeling Thermometer}

Instructions: Use the thermometer below as a scale. Respond to the question by moving the pin next to the thermometer up and down.

Choose the "temperature reading" that best represents your attitudes toward the other mother. Associate warm and favorable attitudes with higher temperatures on the thermometer and associate cold and unfavorable attitudes with lower temperatures on the thermometer.

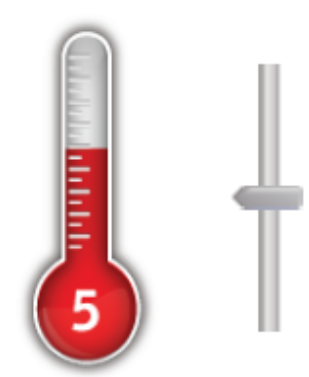




\section{Appendix J}

\section{Representational Mediator Conditions}

\section{Condition \#1: One group recategorization}

Hi! My name is Michelle and I am a mother with two young children. I am the mother Kelly Odenweller paired you with as part of her research study focused on connecting mothers of young children. Kelly asked me to send this email to make an initial connection to you and introduce myself. I'm sure most mothers struggle to develop and maintain adult friendships after kids, but since I became a mother, but I have found it especially challenging to make time for myself and a social life. It is unfortunate that there are not more (convenient) ways to meet other mothers and exchange stories and advice about motherhood. I am really excited to be included in this program so we can help Kelly as she works to build a large network of mothers. I would love to continue this conversation/relationship beyond Kelly's immediate purposes. If you're interested, I'll gladly share my contact information (e.g., email, Facebook, phone) so we can stay in touch. Just ask Kelly after you have finished the study and she will pass my information along to you. Have a great day!

\section{Condition \#2: Two subgroups in one group recategorization}

Hi! My name is Michelle and I am a stay-at-home/working mother with two young children. I am the mother Kelly Odenweller paired you with as part of her research study focused on connecting mothers of young children. Kelly asked me to send this email to make an initial connection to you and introduce myself. I'm sure most mothers struggle to develop and maintain adult friendships after kids, but since I became a stay-at-home/working mother, I have found it especially challenging to make time for myself and a social life. It is unfortunate that there are 
not more (convenient) ways to meet other mothers and exchange stories and advice about motherhood. I am really excited to be included in this program so we can help Kelly as she works to build a large network of mothers. I would love to continue this conversation/relationship beyond Kelly's immediate purposes. If you're interested, I'll gladly share my contact information (e.g., email, Facebook, phone) so we can stay in touch. Just ask Kelly after you have finished the study and she will pass my information along to you. Have a great day!

\section{Condition \#3: Two group categorization}

Hi! My name is Michelle and I am a stay-at-home/working mother with two young children. I am the stay-at-home/working mother Kelly Odenweller paired you with as part of her research study focused on connecting mothers of young children. Kelly asked me to send this email to make an initial connection to you and introduce myself. I'm sure most stay-at-home/working

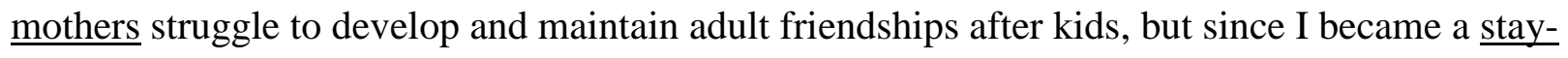
at-home/working mother, I have found it especially challenging to make time for myself and a social life. It is unfortunate that there are not more (convenient) ways to meet other stay-athome/working mothers and exchange stories and advice about being a stay-at-home/working mother. I am really excited to be included in this program so we can help Kelly as she works to build a large network of stay-at-home/working mothers. I would love to continue this conversation/relationship beyond Kelly's immediate purposes. If you're interested, I'll gladly share my contact information (e.g., email, Facebook, phone) so we can stay in touch. Just ask Kelly after you have finished the study and she will pass my information along to you. Have a great day! 


\section{Condition \#4: Separate individuals}

Hi! My name is Michelle. I have been paired with you as part of Kelly Odenweller's research study focused on connecting mothers of young children. Kelly asked me to send this email to make an initial connection to you and introduce myself. I'm sure most people struggle to develop and maintain adult friendships after kids, but I find it especially challenging to make time for myself and a social life. It is unfortunate that there are not more (convenient) ways to meet people and exchange stories and advice about family, friendship, and life in general. I am really excited to be included in this program so we can help Kelly as she works to build a large social network. I would love to continue this conversation/relationship beyond Kelly's immediate purposes. If you're interested, I'll gladly share my contact information (e.g., email, Facebook, phone) so we can stay in touch. Just ask Kelly after you have finished the study and she will pass my information along to you. Have a great day! 
Appendix K

\title{
Study Two Pilot Study Questionnaire
}

\begin{abstract}
Instructions: First, read the following message VERY carefully. This is a message from a stay-at-home mother to a working mother. Then, answer the question on the back based on what you read. Finally, complete the demographic questions.
\end{abstract}

\begin{abstract}
MESSAGE
Hi! My name is Michelle and I am a stay-at-home mother with two young children. I am the stay-at-home mother Kelly Odenweller paired you with as part of her research study focused on connecting mothers of young children. Kelly asked me to send this email to make an initial connection to you and introduce myself. I'm sure most stay-at-home mothers struggle to develop and maintain adult friendships after kids, but since I became a stay-at-home mother, I have found it especially challenging to make time for myself and a social life. It is unfortunate that there are not more (convenient) ways to meet other stay-at-home mothers and exchange stories and advice about being a stay-at-home mother. I am really excited to be included in this program so we can help Kelly as she works to build a large network of stay-at-home mothers. I would love to continue this conversation/relationship beyond Kelly's immediate purposes. If you're interested, I'll gladly share my contact information (e.g., email, Facebook, phone) so we can stay in touch. Just ask Kelly after you have finished the study and she will pass my information along to you. Have a great day!
\end{abstract}

Please continue onto the back for additional questions. 
Choose the one description (from the options below) that you think best represents how a working mother would feel after reading the previous message.

\section{A working mother would feel like she and Michelle are:}

Members of two completely separate groups of stay-at-home and working mothers

Members of two, smaller groups of stay-at-home and working mothers that fall under one larger group of mothers

Members of one, large group of mothers

Unique and separate individuals

Instructions: Please complete the following demographic questions.

1. Your Sex (please circle): Male Female 2. Your age:

3. Please indicate (by circling) the race/ethnicity with which you identify?
a. White
b. Black
c. Asian or Asian American
d. Hispanic or Latino/a
e. Native American
f. Pacific Islander
g. Middle Eastern
h. Bi/Multi-racial or Other? Please specify:

4. Do you have any children (please circle)? Yes No

a. If you have children:

i. Please indicate how many:

ii. Please indicate their ages:

iii. Please indicate if you consider yourself a (please circle):

1. Stay-at-home mother

2. Working mother

3. Father

4. Other:

5. Was your mother a (please circle):
a. Stay-at-home mother
b. Working mother
c. Other: 
Appendix L

Study Two Online Questionnaire 


\section{Dear Participant:}

You are being asked to participate in an IRB-approved research study (Protocol \#1412508170). This research study is being conducted by Dr. Christine E. Rittenour, an Assistant Professor in the Department of Communication Studies at West Virginia University and the principal investigator, and Kelly G. Odenweller, a Ph.D. Candidate/Visiting Instructor in the Department of Communication Studies at West Virginia University and the co-investigator. This research is focused on mothers' adult friendships, communication, and wellbeing. This study will fulfill requirements toward earning a Ph.D. in Communication Studies for the co-investigator.

To qualify for participation, you must be: (a) female, (b) at least 18 years old, (c) have at least one child who is 6 years old or younger. Participation in this study will take approximately $\mathbf{2 0}$ minutes and will proceed in the following two steps:

1. The first step is completing a 10-minute online questionnaire. This questionnaire will ask you to provide basic information about yourself, learn more about the mother with whom you will eventually be interacting, and schedule your online chat session for a day/time that is most convenient for you between January 26 , 2014 and February 13, 2014.

2. The second step is participating in the 10-minute online interaction. On your scheduled date/time, I will join you and the other mother in a Facebook Chat session. I do not anticipate these interactions taking longer than 10 minutes. However, should you and your partner "hit it off" and want to continue your conversation without me, you will be able to exchange personal contact at the end of the study.

Your participation in this study is completely voluntary. You may skip questions on the questionnaire, refrain from interacting in the online chat session, or withdraw your consent at any time. These decisions will not harm your relationship with the researchers or the department/university with which the researchers are affiliated, result in any loss of benefits for which you are otherwise entitled, or affect your relationship with any other mother in the study. Any information about you that is obtained as a result of your participation in this research will be kept as confidential as legally possible. In any conference presentations or publications that result from this research, neither your name nor any information from which you might be identified will be published without your additional consent.

If you do wish to discontinue your participation in the study, please let me know and I can easily and confidentially swap in another mother without your partner knowing you have withdrew. It is MORE important that I receive your honest and candid feedback (whether it's positive or negative) during this process-so please do not feel obligated to withhold your opinions or engage in something with which you are not completely comfortable. No one (including the researchers) will ever be able to connect you to your specific responses as we will keep all of your personal information completely private (even during the actual online chat session). Only should you choose to continue a relationship with the other mother could she have your personal information-but this will be at your discretion. In the event new information becomes available that may affect your willingness to participate in this study, this information will be given to you so that you can make an informed decision about whether or not to continue your participation.

There are no fees required to participate in this study. In exchange for you valuable time and input, all participating mothers will be entered into a drawing for a chance to win one of four $\$ 50$ retailer gift cards. If you withdraw before the end of the study, you will still be eligible for the gift card.

There are no known or expected risks from participating in this study, except for mild frustration or discomfort related to answering questions on the online questionnaire or participating in the online interaction. Should it become necessary, free assistance is available to you through the WVU Carruth Center for Psychological and Psychiatric Services (304-293-4431) or the National Alliance on Mental IIIness (1-800-950-6264).

Because you will have the opportunity to connect with another mother during the online interaction, the potential exists for you to make a new friend. However, similar to your current friendships, you are free to put as much or as little effort into this relationship as you desire and feel comfortable with following the study. Even if you do not receive any direct benefits from this study, the knowledge gained from this study provide insight into mothers' communication and relationships. This information will be invaluable in helping other mothers. 
If you have any questions, concerns, or complaints about this research study, you should contact Dr. Christine E. Rittenour or Kelly G. Odenweller at the emails or phone numbers listed below. If you have questions related to your rights as a research participant, please contact WVU's Office of Research Integrity \& Compliance at (304) 293-7073. Please note that West Virginia University's Institutional Review Board has approval of this study on file (Protocol \#1412508170). If you have read the terms and the conditions of the study and willingly agree to participate in this study, please click the box below to advance to the online questionnaire.

Christine E. Rittenour, Ph.D.

Principal Investigator

Assistant Professor

Department of Communication Studies

West Virginia University

(304) 293-3905

christine.rittenour@mail.wvu.edu

Kelly G. Odenweller, M.A.

Co-Investigator

Ph.D. Candidate/Visiting Instructor

Department of Communication Studies

West Virginia University

(614) 296-5569

kodenwel@mix.wvu.edu

I have read the terms and conditions of this research study and willingly agree to participate.

Your participation in this study begins with the following questionnaire. Please respond to the questions on the following pages as honestly as possible.

Instructions: Please answer the following demographic information about yourself and your children.

Your sex:

Male

Female

Your age:

The number of children you have: 
Your child's/children's age(s):

Note: If you have more than one child, please report all of your children's ages by holding down the control button to select more than one option in the drop down menu below.

\begin{tabular}{|l|}
\hline under 1 month \\
1 month \\
2 months \\
3 months \\
4 months \\
5 months \\
6 months \\
7 months \\
8 months \\
9 months \\
\hline
\end{tabular}

The ethnicity with which you identify:

White (e.g., Caucasian, European, American, Canadian)

Black (e.g., African, African American)

Asian (e.g., Indian, Chinese, Japanese, Korean, Filipino, Vietnamese) or Asian American

Hispanic or Latino/a (e.g., Mexican, Spanish, Puerto Rican, Cuban) or Hispanic/Latin American

Native American (e.g., American Indian, Native Alaskan)

Pacific Islander (e.g., Native Hawaiian, Samoan, Indonesian)

Middle Eastern (e.g., Arab, Turkish, Persian, Somalian, Armenian)

Bi/Multi-racial or Other? Please specify.

Your relationship status:

\section{Single}

Engaged or in a long-term, committed relationship

Married/Remarried

Divorced

Widowed

\section{Your sexual orientation:}

Heterosexual

Gay or Lesbian

Bi-sexual 
Highest level of education you have completed:

Some high school

High school diploma/GED

Professional certificate/license

Some college credits

Bachelor's degree

Graduate degree (e.g., Master's, Ph.D., J.D., MBA)

Your annual household income:

\section{$\boldsymbol{\nabla}$}

Instructions: Please answer the following questions about your identity as a mother.

We are aware that the following labels are often unable to capture the full depth of mothers' work/family arrangements and identities. However, for the purposes of this study, we ask that you select the one that best describes you.

working mother

stay-at-home mother

If you do not identify as working or stay-at-home mother (and felt forced to choose in the previous question), please explain why (in as much detail as possible) these labels are not ideal for you. What term or phrase would best describe your family/work arrangements? 
The image below depicts five potential relationships between you as an individual and stay-at-home/working mothers as a group. Which of the options in the image best represents how SIMILAR you feel to your stay-athome/working mother group? Chose the letter that corresponds to that option, with "A" being the appropriate option if you feel completely different and separate from your stay-at-home/working mother group and " $F$ " being the appropriate option if you feel completely identical to your stay-at-home/working mother group.

$A$
$B$
$C$
$D$
$E$
$F$

Self Mom

Instructions: Using the scale below, indicate your how well each statement describes you.

Does not

describe me AT

ALL
UNDECIDED

as to whether

it describes

me well or

not
Describes me VERY WELL

I have participated in activities that have exposed me to my group of stay-at-home/working mothers. 
I have attended events that have helped me learn more about my group of stay-at-home/working mothers.

I dislike my group of stay-at-home/working mothers.

I am clear about what being a

stay-at-home/working mother means to me.

I understand how I feel about my group of stay-at-home/working mothers.

If I could choose, I would prefer to be of a different mom group (e.g., stay-at-home/working mothers).

I have learned about my group of stay-at-home/ working mothers by doing things such as reading (books, magazines, newspapers), searching the Internet, or keeping up with current events.

I wish I were of a different mom group (e.g.,stay-athome/working mothers).

I am not happy with my group of stay-at-home/ working mothers.

I have read books, magazine, newspapers, or other materials that have taught me about my group of stay-at-home/working mothers.

I feel negatively about my group of stay-athome/working mothers.

I have a clear sense of what being a stay-at-home/ working mothers means to me.

I have not participated in any activities that would teach me about my group of stay-at-home/ working mothers.

My feelings about my group of stay-at-home/ working mothers are mostly negative.

I have participated in activities that have taught me about my group of stay-at-home/working mothers.

I have experienced things that reflect my group of stay-at-home/working mothers, such as reading books, watching movies, or attending events.

The questions on the next four pages will ask you about your experiences interacting with stay-at-home mothers, working mothers, and non-mothers (i.e., men and women who are NOT parents). 
Instructions: Using the scale below, indicate your level of agreement with each statement in relation.

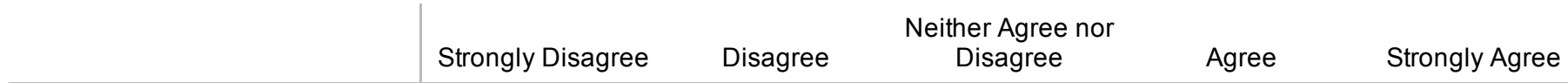

All of my friends and $\mathrm{I}$ have similar work/family

arrangements.

I have close friends who are stay-at-home mothers.

I speak to stay-at-home mothers on a regular basis.

I have at least one acquaintance who is a stay-athome mother.

I have never talked to a stay-athome mother.

I have close friends who are working mothers.

I speak to working mothers on a regular basis.

I have at least one acquaintance who is a working mother.

I have never talked to a working mother.

I have close friends who are not mothers.

I speak to people who are not mothers on a regular basis.

I have at least one acquaintance who is not a mother.

I have never talked to someone who is not a mother.

Instructions: The questions and scales on this page address your past interactions with STAY-AT-HOME mothers. As a note, the same question will be repeated five times, but the scale below the question changes each time to assess different aspects of these interactions. Please respond to each scale individually.

When you came in contact with stay-at-home mothers in the past, overall you perceived your interactions to be:

Imbalanced in terms of

Balanced in terms power/status 
When you came in contact with stay-at-home mothers in the past, overall you perceived your Involuntary

When you came in contact with stay-at-home mothers in the past, overall you perceived your interactions to be:

Superficial

Intimate

When you came in contact with stay-at-home mothers in the past, overall you perceived your interactions to be:

Competitive

Cooperative

When you came in contact with stay-at-home mothers in the past, overall you perceived your interactions to be:

Uncomfortable

Pleasant

Instructions: The questions and scales on this page address your past interactions with WORKING mothers. As a note, the same question is repeated five times, but the scale below the question changes each time to assess different aspects of these interactions. Please respond to each scale individually.

When you came in contact with working mothers in the past, overall you perceived your interactions to be:

$$
\begin{gathered}
\text { Imbalanced in } \\
\text { terms of } \\
\text { power/status }
\end{gathered}
$$

When you came in contact with working mothers in the past, overall you perceived your interactions to be:

Involuntary

Voluntary

When you came in contact with working mothers in the past, overall you perceived your interactions to be: 
When you came in contact with working mothers in the past, overall you perceived your interactions to be:

Competitive

Cooperative

When you came in contact with working mothers in the past, overall you perceived your interactions to be:

Uncomfortable

Pleasant

Instructions: The questions and scales on this page address your past interactions with NON-MOTHERS. As a note, the same question is repeated five times, but the scale below the question changes each time to assess different aspects of these interactions. Please respond to each scale individually.

When you came in contact with non-mothers in the past, overall you perceived your interactions to be:

$$
\begin{gathered}
\text { Imbalanced in } \\
\text { terms of } \\
\text { power/status }
\end{gathered}
$$

Balanced in terms of power/status

When you came in contact with non-mothers in the past, overall you perceived your interactions to be: Involuntary

Voluntary

When you came in contact with non-mothers in the past, overall you perceived your interactions to be:

Superficial

Intimate

When you came in contact with non-mothers in the past, overall you perceived your interactions to be:

Competitive

Cooperative

When you came in contact with non-mothers in the past, overall you perceived your interactions to be: 
I have less time to maintain adult friendships than I did before I had children.

$\begin{array}{ccccc} & & \text { Somewhat } & \text { Neither Agree nor } & \\ \text { Strongly Disagree } & \text { Disagree } & \text { Disagree } & \text { Disagree } & \text { Somewhat Agree }\end{array}$

I have less interest in maintaining adult friendships than I did before I had children.

\begin{tabular}{|c|c|c|c|c|}
\hline Strongly Disagre & Disagree & $\begin{array}{c}\text { Somewhat } \\
\text { Disaqree }\end{array}$ & $\begin{array}{c}\text { Neither Agree nor } \\
\text { Disagree }\end{array}$ & Somewhat Agree \\
\hline
\end{tabular}

It is easier to maintain adult friendship through online channels (e.g., email, Facebook, instant messenger) compared to face-to-face or over the phone.

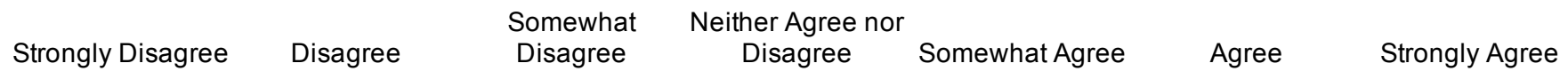

I wish I could maintain more adult friendships than I currently do.

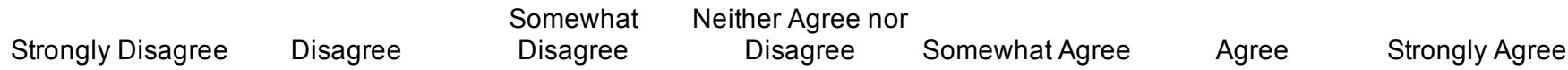

Before we randomly pair you with another mother involved in this project, please write a brief message to this mother. In this message you can introduce yourself and share any details about your children, your partner/spouse, your mother-in-law, etc. that you would like us to pass along to the other mother. This is also a great place to discuss what you like to do with your free time, your favorite hobbies/leisure activities, what you do to unwind and relax, what you would love to do if you had a babysitter tonight, etc. The other mother has been asked to do this as well and, once you complete this section, you will have the opportunity to read her message to you. 
On the next page, you will see a brief message from a stay-at-home/working mother who has been randomly selected to join us in the 10-minute online chat session. Please read her message carefully before advancing to the folowing page

Hi! My name is Michelle and I am a mother with two young children. I am the mother Kelly Odenweller paired you with as part of her research study focused on connecting mothers of young children. Kelly asked me to send this brief message to introduce myself. I'm sure most mothers struggle to develop and maintain adult friendships after kids, but since I became a mother, but I have found it especially challenging to make time for myself and a social life. It is unfortunate that there are not more (convenient) ways to meet other mothers and exchange stories and advice about motherhood. I am really excited to be included in this project so we can help Kelly as she works to build a large network of mothers. I would love to continue this conversation/relationship beyond Kelly's immediate purposes. If you're interested, I'll gladly share my contact information (e.g., email, Facebook, phone) so we can stay in touch. Just ask Kelly after you have finished the study and she will pass my information along to you. Have a great day!

Instructions: Please answer the following questions about the mother whose message you read on the previous page (and who you will eventually interact with in the online chat session).

Did you carefully read the other mothers' message?

yes

no

After reading the other mothers' message, I feel as if we are:

members of one large group of mothers

members of two, smaller subgroups of stay-at-home and working mothers that fall under one larger group of mothers

unique and separate individuals

members of two completely separate groups of stay-at-home and working mothers 


\section{The other mother is a:}

working mother

stay-at-home mother

Instructions: Using the following thermometer as a scale, indicate how you feel about the mother with which you have been randomly paired. While completing this instrument, associate warm and favorable attitudes with higher temperatures on the thermometers and associate cold and unfavorable attitudes with lower temperatures on the thermometers. Choose the "temperature reading" that best represent your attitudes toward the other mother.
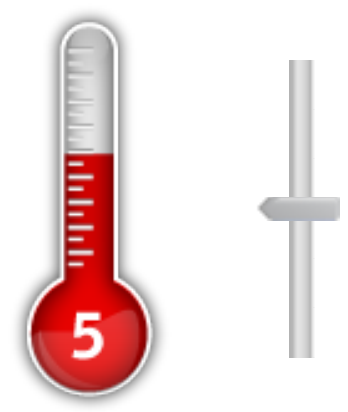

Instructions: Using the scale below, indicate your level of agreement with each statement.

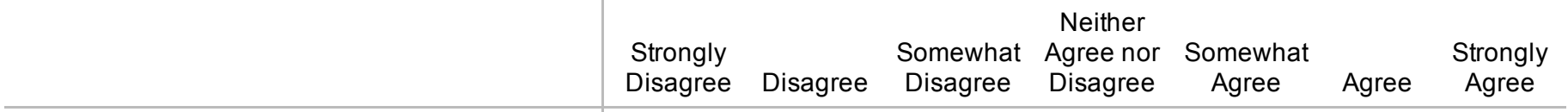

The other mother is different from other stay-at-home/working mothers.

The other mother is a typical stay-at-home/working mothers.

The other mother is similar to other stay-at-home/working mothers. 
Instructions: Using the scale below, indicate how well you think each statement describes the other mother based on what you read in her message.

\begin{tabular}{|c|c|c|c|c|c|c|c|}
\hline & $\begin{array}{l}\text { Does not } \\
\text { describe her } \\
\text { AT ALL }\end{array}$ & & & & & & $\begin{array}{c}\text { Describes } \\
\text { her } \\
\text { EXACTLY }\end{array}$ \\
\hline Flexible and family-oriented & ○ & $\bigcirc$ & $\bigcirc$ & $\bigcirc$ & $\bigcirc$ & $\bigcirc$ & $\odot$ \\
\hline Busy and overworked & $\bigcirc$ & $\bigcirc$ & $\bigcirc$ & 0 & $\bigcirc$ & $\bigcirc$ & $\bigcirc$ \\
\hline Traditional & 0 & $\bigcirc$ & 0 & 0 & 0 & 0 & 0 \\
\hline Balancing work, family, and life & $\bigcirc$ & $\bigcirc$ & ○ & $\bigcirc$ & $\bigcirc$ & $\bigcirc$ & 0 \\
\hline A supermom & $\bigcirc$ & $\bigcirc$ & 0 & $\bigcirc$ & 0 & $\bigcirc$ & $\bigcirc$ \\
\hline Non-traditional & ○ & $\bigcirc$ & 0 & 0 & 0 & 0 & ○ \\
\hline An executive of her home & 0 & 0 & 0 & 0 & 0 & 0 & 0 \\
\hline Hardworking and determined & $\bigcirc$ & 0 & 0 & $\bigcirc$ & $\bigcirc$ & $\bigcirc$ & ○ \\
\hline Lazy, selfish, and lost & 0 & 0 & 0 & $\bigcirc$ & 0 & 0 & 0 \\
\hline Helicopter parent & 0 & $\bigcirc$ & 0 & $\bigcirc$ & 0 & 0 & $\bigcirc$ \\
\hline An ideal mother & $\bigcirc$ & $\bigcirc$ & 0 & $\bigcirc$ & 0 & $\bigcirc$ & $\bigcirc$ \\
\hline
\end{tabular}

In addition to other challenges mothers face related to forming and maintaining adult relationships, it is difficult to find a good babysitter. The other mother with which you have been paired is currently looking for help finding a babysitter for an upcoming night out. In the boxes provided below, please list as many ideas, resources, or even specific names/contact information that can help the other mother secure a good babysitter.

Instructions: Using the scale below, indicate your level of agreement with each statement.

I would like to participate in a program to help build connections between stay-at-home and working mothers.

Strongly Disagree Disagree $\quad \begin{gathered}\text { Somewhat } \\ \text { Disagree }\end{gathered} \quad \begin{gathered}\text { Neither Agree nor } \\ \text { Disagree }\end{gathered} \quad$ Somewhat Agree $\quad$ Agree $\quad$ Strongly Agree

I would be willing to speak to the other mother in an online chat session.

Strongly Disagree Disagree $\quad \begin{gathered}\text { Somewhat } \\ \text { Disagree }\end{gathered} \quad \begin{gathered}\text { Neither Agree nor } \\ \text { Disagree }\end{gathered} \quad$ Somewhat Agree $\quad$ Agree $\quad$ Strongly Agree


I would be willing to connect with the other mother on social media (e.g., Facebook, Twitter).

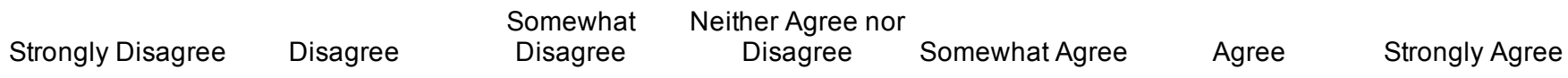

I would be willing to meet the other mother face-to-face.

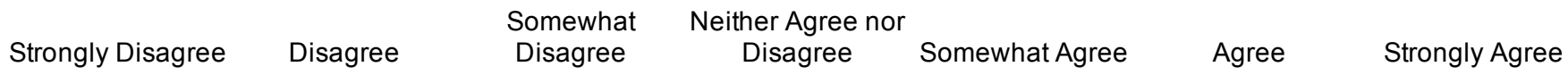

Thank you for completing the previous questions. Please advance to the next page to schedule your online chat session with the other mother. If-for whatever reason-you do not wish to interact with the other mother, please click "exit study now" below.

Schedule online chat session now

Exit study now

Please choose the option below that BEST explains why you chose to exit the survey now (i.e., before participating in the online interaction). Please note the other mother will NEVER see this response. Additionally, your response will not jeopardize your entrance into the raffle for the $\$ 50$ gift card. I simply want to know what you are thinkingplease be honest and candid.

I no longer have time to participate in the 10-minute online interaction

I do not feel comfortable talking in online chat sessions

I do not want to interact with the specific mother you have paired me with

I am worried the other mother will not like me

Other (please specify):

Thank you so much for your time and input! You have now reached the end of the study. So that you could best help with my goal of connecting mothers, I withheld some information from you at the beginning of the study. I would like to share that information with you now.

Ultimately, my research is devoted to minimizing what the media has labeled the "mommy wars," intense rivalries among mothers based on a variety of parenting decisions and family lifestyles. You may have watched a news program or read an article about, or even personally experienced, these "wars" as a mother. My dissertation (WVU IRB Protocol \#1412508170) is focused specifically on stay-at-home and working mothers, and I am using the data you have just provided to help me understand the attitudes, feelings, and communication between these women.

Initially, I said you would be participating in an online interaction with another mother, from whom you received a brief message. Neither the mother nor the message were real. Both were constructed from previous research on group boundaries. The questions you answered about this mother comprised the entire data collection. Again, this data will be used to inform future efforts to move from anxious or avoidant communication (i.e., "mommy wars") to more inclusive and supportive communication among mothers. Now that you have provided this confidential data, 
you may engage in what you set out to do, and what was advertised in my initial message: connect with other mothers.

I have created a private Facebook group where you can join all of the mothers who generously gave their time for this study and begin interacting with them as you likely hoped to do when you signed on for this study. There is NO obligation to join this group and this Facebook page is NOT part of the study (i.e., nothing you post on this page may be used for research purposes). Aside from posting my own/participating in your conversations about motherhood, family, work, and life, I will use this group to make announcements about the winners of the $\$ 50$ gift cards, results of my recent research studies, and future research opportunities. If you are interested, please follow the link here: Comma Mommas.

As a reminder, the anonymous responses you provided will never be connected to you as not even I know who shared which responses. However, if you would like your data to be withdrawn from the study, please let me know. Additionally, if required, free assistance is available to you through the WVU Carruth Center for Psychological and Psychiatric Services (304-293-4431) or the National Alliance on Mental IIIness (1-800-950-6264).

As I will be collecting data for my dissertation until February 13, 2014 and would like to preserve the integrity of my study design, I would greatly appreciate it if you did not share this information with other mothers who might become future participants. I will announce the closing of the study via the Facebook group mentioned above. At that time, you will be completely free to discuss your participation publicly. If you would like more information regarding this research project now, please contact me privately at kodenwel@mix.wvu.edu or 614-296-5569. Thank you again for your involvement in my dissertation. I wish you and yours a fabulous 2015!

Sincerely,

Kelly

Christine E. Rittenour, Ph.D.

Principal Investigator

Assistant Professor

Department of Communication Studies

West Virginia University

(304) 293-3905

christine.rittenour@mail.wvu.edu

Kelly G. Odenweller, M.A.

Co-Investigator

Ph.D. Candidate/Visiting Instructor

Department of Communication Studies

West Virginia University

(614) 296-5569

kodenwel@mix.wvu.edu 